\title{
Nickel-Catalyzed Chemoselective Asymmetric Hydrogenation of $\alpha, \beta$-Unsaturated Ketoimines: An Efficient Approach to Chiral Allylic Amines
}

Xiang Zhao, Feng Zhang, Kai Liu, Xumu Zhang, Hui Lv

\section{Contents}

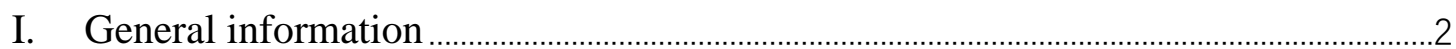

II. General procedure for the synthesis of $\alpha, \beta$-unsaturated ketoimines ............................

III. Procedure for asymmetric hydrogenation of $\alpha, \beta$-unsaturated ketoimines ................ 12

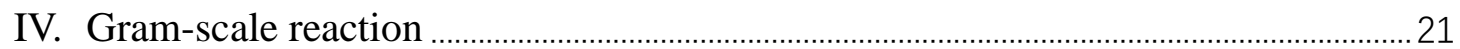

V. Reference.

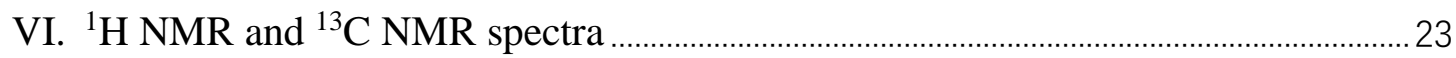

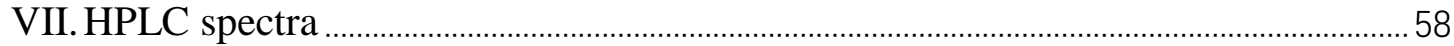




\section{General information}

Unless otherwise noted, all reagents and solvents were purchased from commercial suppliers and used without further purification. NMR spectra were recorded on Bruker ADVANCE III (400 MHz) spectrometers for ${ }^{1} \mathrm{H}$ NMR and ${ }^{13} \mathrm{C}$ NMR. $\mathrm{CDCl}_{3}$ was the solvent used for the NMR analysis, with tetramethylsilane as the internal standard. Chemical shifts were reported upfield to TMS $(0.00 \mathrm{ppm})$ for ${ }^{1} \mathrm{H}$ NMR and relative to $\mathrm{CDCl}_{3}(77.16 \mathrm{ppm})$ for ${ }^{13} \mathrm{C} \mathrm{NMR}$. Optical rotation was determined using a Perkin Elmer 343 polarimeter. HPLC analysis was conducted on an Agilent 1260 Series instrument. Column Chromatography was performed with silica gel Merck 60 (300-400 mesh). All new products were further characterized by HRMS. A positive ion mass spectrum of sample was acquired on a Thermo LTQ-FT mass spectrometer with an electrospray ionization source. 


\section{General procedure for the synthesis of $\alpha, \beta$-unsaturated ketoimines}

A)
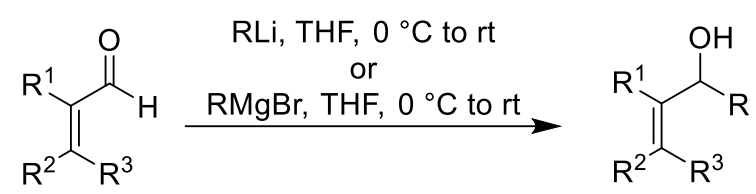<smiles>[R]C(=O)C([R])=C([R])[R]</smiles>

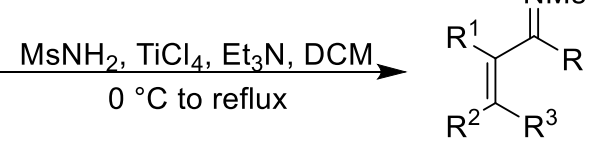

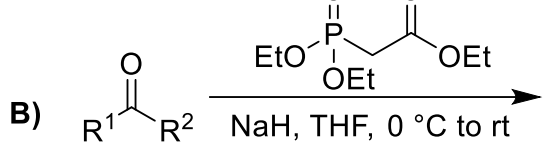<smiles>[R]C([R])=CC(=O)OCC</smiles>

$\underset{\mathrm{MeNH}(\mathrm{OMe}) \cdot \mathrm{HCl}, i-\mathrm{PrMgCl}}{\stackrel{\mathrm{THF}}{ }-8^{\circ} \mathrm{C} \text { to } 0{ }^{\circ} \mathrm{C}}$<smiles>[R]/[R](=C/C(=O)N(C)OC)C([R])=O</smiles>

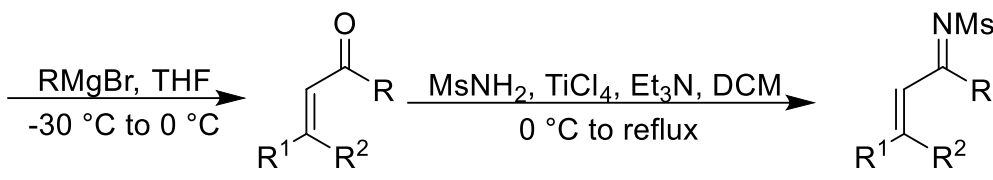

C)

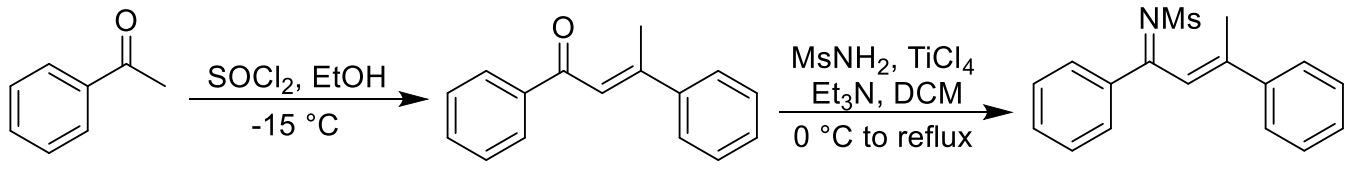

\section{Method A for the synthesis of $\alpha, \beta$-unsaturated ketoimines.}

To a solution of 3-methyl-2-butenal in THF, $\mathrm{PhMgBr}$ (1.1 equiv) was added at $0{ }^{\circ} \mathrm{C}$. The mixture was then stirred for $2 \mathrm{~h}$ at room temperature. Subsequently, the reaction was quenched with water and extracted with EtOAc. The combined organic layers were washed with brine, dried over $\mathrm{Na}_{2} \mathrm{SO}_{4}$ and concentrated in vacuo. The crude product was purified by column chromatography on silica gel to afford 3-methyl-1phenylbut-2-en-1-ol. ${ }^{[1-2]}$

To a solution of 3-methyl-1-phenylbut-2-en-1-ol in $\mathrm{Et}_{2} \mathrm{O}$ was added $\mathrm{MnO}_{2}$ (10 equiv.). The mixture was then stirred for $12 \mathrm{~h}$ at room temperature. Subsequently, the reaction was filtered and the solvent was removed. The crude product was purified by column chromatography on silica gel to afford 3-methyl-1-phenylbut-2-en-1-one. ${ }^{[1-2]}$

To a mixture of 3-methyl-1-phenylbut-2-en-1-one, methanesulfonamide (1 equiv.) and $\mathrm{Et}_{3} \mathrm{~N}$ (2.4 equiv.) in $\mathrm{CH}_{2} \mathrm{Cl}_{2}$ at $0{ }^{\circ} \mathrm{C}$, was slowly added $\mathrm{TiCl}_{4}$ (1.2 equiv.). The 
reaction mixture was initially stirred at $0{ }^{\circ} \mathrm{C}$ for $0.5 \mathrm{~h}$ and then at reflux temperature for 12 h. After complete consumption of the ketone substrate (monitored by TLC), the reaction mixture was diluted with water and the organic layer was separated. The aqueous layer was extracted with $\mathrm{CH}_{2} \mathrm{Cl}_{2}$ and the combined organics were washed with water and dried over anhydrous $\mathrm{Na}_{2} \mathrm{SO}_{4}$. After removal of the organic solvent under vacuum, the residue was purified by flash column chromatography on silica gel (eluent: hexanes/EtOAc) to afford the desired imine. ${ }^{[9-16]}$ The product can be further purified by recrystallization from ethanol. Substrates 10 were prepared through method A.

\section{Method B for the synthesis of $\alpha, \beta$-unsaturated ketoimines.}

To a suspension of $\mathrm{NaH}$ (60\% dispersion in oil, $12.0 \mathrm{mmol})$ in THF (10 mL) was added a solution of triethyl phosphonoacetate $(12.0 \mathrm{mmol})$ in THF $(5 \mathrm{~mL})$ under a nitrogen atmosphere at $0{ }^{\circ} \mathrm{C}$. After being stirred at room temperature for $30 \mathrm{~min}$, a solution of a corresponding ketone $(10 \mathrm{mmol})$ in THF $(5 \mathrm{~mL})$ was added to the reaction mixture at $0{ }^{\circ} \mathrm{C}$. After being further stirred at room temperature for $16 \mathrm{~h}$, the reaction was quenched by adding saturated aqueous $\mathrm{NaHCO}_{3}$ and diluted with EtOAc. The organic layer was separated and washed with brine, dried over $\mathrm{MgSO}_{4}$ and concentrated at reduced pressure. The residue was purified by silica gel column chromatography to afford the corresponding $\alpha, \beta$-unsaturated ester. ${ }^{[4-8]}$

To a suspension of the $\alpha, \beta$-unsaturated ester $(3.0 \mathrm{mmol})$ obtained above and $\mathrm{N}, \mathrm{O}$ dimethylhydroxylamine hydrochloride $(6.0 \mathrm{mmol})$ in THF $(6.0 \mathrm{~mL})$ was added dropwise $i$ - $\mathrm{PrMgCl}(2.0 \mathrm{M}$ solution in THF, $6.0 \mathrm{mmol})$ under nitrogen atmosphere at 5 to $-10{ }^{\circ} \mathrm{C}$. After being stirring at $0{ }^{\circ} \mathrm{C}$ for $30 \mathrm{~min}$, the reaction was quenched by adding saturated aqueous $\mathrm{NH}_{4} \mathrm{Cl}$ and diluted with EtOAc. The organic layer was separated and washed with brine, dried over $\mathrm{MgSO}_{4}$ and concentrated at reduced pressure. The residue was purified by silica gel column chromatography to afford the corresponding Weinreb amide.

To a solution of the Weinreb amide $(1.0 \mathrm{mmol})$ obtained above in THF $(2.0 \mathrm{~mL})$ was added dropwise a solution of the corresponding Grignard reagent or lithium reagent 
( $1.5 \mathrm{mmol}$ ) under nitrogen atmosphere at $-30{ }^{\circ} \mathrm{C}$. After being stirring at $0{ }^{\circ} \mathrm{C}$ for $30 \mathrm{~min}$, the reaction was quenched by adding saturated aqueous $\mathrm{NH}_{4} \mathrm{Cl}$ and diluted with EtOAc. The organic layer was separated and washed with brine, dried over $\mathrm{MgSO}_{4}$ and concentrated at reduced pressure. The residue was purified by silica gel column chromatography to afford the $\beta, \beta$-disubstituted enone.

To a mixture of $\beta$, $\beta$-disubstituted enone, methanesulfonamide (1 equiv.) and $\mathrm{Et}_{3} \mathrm{~N}$ (2.4 equiv.) in $\mathrm{CH}_{2} \mathrm{Cl}_{2}$ at $0{ }^{\circ} \mathrm{C}$, was slowly added $\mathrm{TiCl}_{4}$ (1.2 equiv.). The reaction mixture was initially stirred at $0{ }^{\circ} \mathrm{C}$ for $0.5 \mathrm{~h}$ and then at reflux temperature for $12 \mathrm{~h}$. After complete consumption of the ketone substrate (monitored by TLC), the reaction mixture was diluted with water and the organic layer was separated. The aqueous layer was extracted with $\mathrm{CH}_{2} \mathrm{Cl}_{2}$ and the combined organics were washed with water and dried over anhydrous $\mathrm{Na}_{2} \mathrm{SO}_{4}$. After removal of the organic solvent under vacuum, the residue was purified by flash column chromatography on silica gel (eluent: hexanes/EtOAc) to afford the desired imine. ${ }^{[9-16]}$ The product can be further purified by recrystallization from ethanol. Substrates 1d-1n were prepared through method B.

\section{Method $\mathrm{C}$ for the synthesis of $\alpha, \beta$-unsaturated ketoimines.}

To a stirred mixture of the ketones $(15 \mathrm{mmol})$ and anhydrous ethanol $(75 \mathrm{mmol})$, sulfurous oxychloride $(25 \mathrm{mmol})$ was added. When the solution turned deep red, saturation $\mathrm{Na}_{2} \mathrm{CO}_{3}$ was added and the mixture was extracted twice with ether $(20 \mathrm{~mL})$. The organic layer was separated and dried over anhydrous $\mathrm{Na}_{2} \mathrm{SO}_{4}$. After evaporation of the solvent under reduced pressure, the residue was purified by column chromatography on silica gel using petroleum ether/acetone (95/5) as the eluent, giving the pure dypnone. ${ }^{[3]}$

To a mixture of dypnone, methanesulfonamide (1 equiv.) and $\mathrm{Et}_{3} \mathrm{~N}$ (2.4 equiv.) in $\mathrm{CH}_{2} \mathrm{Cl}_{2}$ at $0{ }^{\circ} \mathrm{C}$, was slowly added $\mathrm{TiCl}_{4}$ (1.2 equiv.). The reaction mixture was initially stirred at $0{ }^{\circ} \mathrm{C}$ for $0.5 \mathrm{~h}$ and then at reflux temperature for $12 \mathrm{~h}$. After complete consumption of the ketone substrate (monitored by TLC), the reaction mixture was diluted with water and the organic layer was separated. The aqueous layer was extracted 
with $\mathrm{CH}_{2} \mathrm{Cl}_{2}$ and the combined organics were washed with water and dried over anhydrous $\mathrm{Na}_{2} \mathrm{SO}_{4}$. After removal of the organic solvent under vacuum, the residue was purified by flash column chromatography on silica gel (eluent: hexanes/EtOAc) to afford the desired imine. ${ }^{[9-16]}$ The product can be further purified by recrystallization from ethanol. Substrates 1a, 1b, 1c, 1p, 1q were prepared through method C.

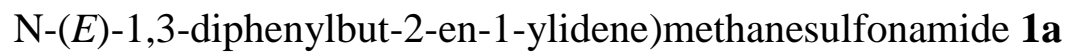<smiles>C/C(=C\C(c1ccccc1)=[N+](C)C)c1ccccc1</smiles>

White solid; M. P. $104-106{ }^{\circ} \mathrm{C},{ }^{1} \mathrm{H}$ NMR (400 MHz, $\mathrm{CDCl}_{3}$ ), $\delta$ (ppm) 8.00-7.91 (m, 2H), $7.59(\mathrm{~m}, 3 \mathrm{H}), 7.51-7.35(\mathrm{~m}, 5 \mathrm{H}), 7.07(\mathrm{~s}, 1 \mathrm{H}), 3.24(\mathrm{~s}, 3 \mathrm{H}), 1.90(\mathrm{~s}, 3 \mathrm{H}) ;{ }^{13} \mathrm{C}$ NMR (100 MHz, $\mathrm{CDCl}_{3}$ ), $\delta$ (ppm) 178.61, 146.11, 140.92, 136.93, 133.44, 129.66, 128.91, 128.89, 128.61, 126.37, 121.33, 42.91, 19.62. ESI-HRMS calculated for $\mathrm{C}_{17} \mathrm{H}_{17} \mathrm{NO}_{2} \mathrm{SNa}^{+}\left([\mathrm{M}+\mathrm{Na}]^{+}\right)$: 322.0872; Found: 322.0867 .

$\mathrm{N}-(E)$-1,3-diphenylbut-2-en-1-ylidene)-N, N-dimethyl-methanesulfonamide $\mathbf{1 b}$<smiles>COS(=O)(=O)C(/C=C(\C)c1ccccc1)c1ccccc1</smiles>

Yellow solid; M. P. $132-134{ }^{\circ} \mathrm{C},{ }^{1} \mathrm{H}$ NMR (400 MHz, $\left.\mathrm{CDCl}_{3}\right), \delta(\mathrm{ppm})$ 8.01-7.89 $(\mathrm{m}, 2 \mathrm{H}), 7.63-7.53(\mathrm{~m}, 3 \mathrm{H}), 7.46(\mathrm{t}, J=7.6 \mathrm{~Hz}, 2 \mathrm{H}), 7.43-7.31(\mathrm{~m}, 3 \mathrm{H}), 7.01(\mathrm{~d}, J=$ $1.4 \mathrm{~Hz}, 1 \mathrm{H}), 2.95$ (s, 6H), 1.90 (s, 3H); ${ }^{13} \mathrm{C} \mathrm{NMR}\left(100 \mathrm{MHz}, \mathrm{CDCl}_{3}\right), \delta$ (ppm) 178.80, $145.20,141.10,137.35,133.13,129.47,128.86,128.74,128.59,126.34,121.96,38.93$, 19.54. ESI-HRMS calculated for $\mathrm{C}_{18} \mathrm{H}_{20} \mathrm{~N}_{2} \mathrm{O}_{2} \mathrm{SNa}^{+}\left([\mathrm{M}+\mathrm{Na}]^{+}\right)$: 351.1138; Found: 351.1135 .

$\mathrm{N}$-(E)-1,3-diphenylbut-2-en-1-ylidene)-4-methylbenzenesulfonamide 1c 
<smiles>C/C(=C\C(=[N+])c1ccccc1)c1ccccc1</smiles>

Faint yellow solid; M. P. $119-121{ }^{\circ} \mathrm{C},{ }^{1} \mathrm{H}$ NMR (400 MHz, $\left.\mathrm{CDCl}_{3}\right), \delta$ (ppm) 7.91 (dd, $J=13.8,8.1 \mathrm{~Hz}, 4 \mathrm{H}), 7.62-7.52(\mathrm{~m}, 3 \mathrm{H}), 7.47-7.35(\mathrm{~m}, 5 \mathrm{H}), 7.32-7.24(\mathrm{~m}, 2 \mathrm{H})$, 7.06 (s, 1H), 2.42 (s, 3H), 1.78 (s, 3H); ${ }^{13} \mathrm{C}$ NMR (100 MHz, $\left.\mathrm{CDCl}_{3}\right), \delta$ (ppm) 178.43, $145.30,143.52,140.75,138.29,137.00,133.49,129.83,129.42,128.88,128.83$, $128.63,127.48, \quad 126.26,121.75, \quad 21.64,19.33$. ESI-HRMS calculated for $\mathrm{C}_{23} \mathrm{H}_{21} \mathrm{NO}_{2} \mathrm{SNa}^{+}\left([\mathrm{M}+\mathrm{Na}]^{+}\right)$: 398.1185; Found: 398.1185 .

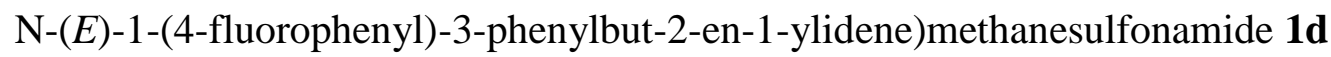<smiles>C/C(=C\C(c1ccccc1)=[N+]([O-])c1ccc(F)cc1)c1ccccc1</smiles>

Faint yellow solid; M. P. 106-108 ${ }^{\circ} \mathrm{C},{ }^{1} \mathrm{H}$ NMR (400 MHz, CDCl 3 ), $\delta(\mathrm{ppm}) 7.99$ $(\mathrm{dd}, J=8.7,5.6 \mathrm{~Hz}, 2 \mathrm{H}), 7.63-7.55(\mathrm{~m}, 2 \mathrm{H}), 7.45-7.35(\mathrm{~m}, 3 \mathrm{H}), 7.20-7.11(\mathrm{~m}, 2 \mathrm{H})$, 7.05 - $6.98(\mathrm{~m}, 1 \mathrm{H}), 3.24$ (s, 3H), 1.98-1.85 (s, 3H); ${ }^{13} \mathrm{C} \mathrm{NMR}\left(100 \mathrm{MHz}, \mathrm{CDCl}_{3}\right), \delta$ (ppm) 177.19, 166.17 (d, $J=254 \mathrm{~Hz}), 145.89,140.71,132.99,132.30(\mathrm{~d}, J=10 \mathrm{~Hz})$, 128.96, 128.65, 126.33, 121.04, 116.19 (d, $J=22 \mathrm{~Hz}), 42.88$, 19.56. ESI-HRMS calculated for $\mathrm{C}_{17} \mathrm{H}_{16} \mathrm{FNO}_{2} \mathrm{SNa}^{+}\left([\mathrm{M}+\mathrm{Na}]^{+}\right)$: 340.0778; Found: 340.0774 .

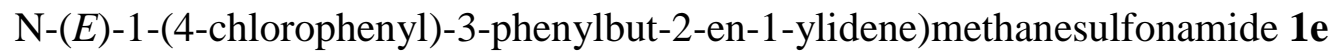<smiles>CN=C(/C=C(\C)c1ccccc1)c1ccc(Cl)cc1</smiles>

Brown solid; M. P. 105-107 ${ }^{\circ} \mathrm{C},{ }^{1} \mathrm{H}$ NMR (400 MHz, $\left.\mathrm{CDCl}_{3}\right), \delta(\mathrm{ppm}) 7.90(\mathrm{~d}, J$ $=8.4 \mathrm{~Hz}, 2 \mathrm{H}), 7.63-7.53(\mathrm{~m}, 2 \mathrm{H}), 7.48-7.36(\mathrm{~m}, 5 \mathrm{H}), 7.05-6.99(\mathrm{~m}, 1 \mathrm{H}), 3.24(\mathrm{~s}, 3 \mathrm{H})$, 1.90 (s, 3H); ${ }^{13} \mathrm{C}$ NMR (100 MHz, $\mathrm{CDCl}_{3}$ ), $\delta$ (ppm) 177.29, 146.29, 140.66, 140.02, 135.28, 130.96, 129.28, 129.03, 128.66, 126.34, 120.85, 42.89, 19.65. ESI-HRMS calculated for $\mathrm{C}_{17} \mathrm{H}_{16} \mathrm{ClNO}_{2} \mathrm{SNa}^{+}\left([\mathrm{M}+\mathrm{Na}]^{+}\right)$: 356.0474 ; Found: 356.0482 . 
$\mathrm{N}-(E)-1-(4-m e t h o x y p h e n y l)-3-p h e n y l b u t-2-e n-1-y l i d e n e) m e t h a n e s u l f o n a m i d e ~ \mathbf{~} f$<smiles></smiles>

Yellow solid; M. P. $114-116{ }^{\circ} \mathrm{C},{ }^{1} \mathrm{H}$ NMR $\left(400 \mathrm{MHz}, \mathrm{CDCl}_{3}\right), \delta$ (ppm) 7.95 (d, J $=8.9 \mathrm{~Hz}, 2 \mathrm{H}), 7.63-7.55(\mathrm{~m}, 2 \mathrm{H}), 7.43-7.32(\mathrm{~m}, 3 \mathrm{H}), 7.01-6.92(\mathrm{~m}, 3 \mathrm{H}), 3.89(\mathrm{~s}, 3 \mathrm{H})$, $3.21(\mathrm{~s}, 3 \mathrm{H}), 1.93(\mathrm{~s}, 3 \mathrm{H}) ;{ }^{13} \mathrm{C} \mathrm{NMR}\left(100 \mathrm{MHz}, \mathrm{CDCl}_{3}\right), \delta(\mathrm{ppm}) 177.66,164.25,144.57$, 140.96, 132.05, 129.03, 128.68, 128.58, 126.31, 121.52, 114.27, 55.62, 42.86, 19.35. ESI-HRMS calculated for $\mathrm{C}_{18} \mathrm{H}_{19} \mathrm{NO}_{3} \mathrm{SNa}^{+}\left([\mathrm{M}+\mathrm{Na}]^{+}\right)$: 352.0978 ; Found: 352.0977.

$\mathrm{N}$-(E)-3-phenyl-1-(p-tolyl)but-2-en-1-ylidene)methanesulfonamide $\mathbf{1 g}$<smiles>CN=C(/C=C(\C)c1ccccc1)c1ccc(C)cc1</smiles>

Brown solid; M. P. 96-98 ${ }^{\circ} \mathrm{C},{ }^{1} \mathrm{H}$ NMR $\left(400 \mathrm{MHz}, \mathrm{CDCl}_{3}\right), \delta(\mathrm{ppm}) 7.86(\mathrm{~d}, J=$ $8.0 \mathrm{~Hz}, 2 \mathrm{H}), 7.59$ (dd, $J=6.0,1.6 \mathrm{~Hz}, 2 \mathrm{H}), 7.43-7.35(\mathrm{~m}, 3 \mathrm{H}), 7.28(\mathrm{~s}, 2 \mathrm{H}), 7.02(\mathrm{~s}$, 1H), 3.23 (s, 3H), 2.44 (s, 3H), 1.91 (s, 3H); $\left.{ }^{13} \mathrm{C} \mathrm{NMR} \mathrm{(100} \mathrm{MHz,} \mathrm{CDCl}_{3}\right), \delta(\mathrm{ppm})$ $178.45,145.36,144.63,140.97,134.10,129.79$, 129.68, 128.78, 128.59, 126.34, 121.47, 42.89, 21.79, 19.51. ESI-HRMS calculated for $\mathrm{C}_{18} \mathrm{H}_{19} \mathrm{NO}_{2} \mathrm{SNa}^{+}\left([\mathrm{M}+\mathrm{Na}]^{+}\right)$: 336.1029; Found: 336.1025.

N-(1E)-1-(3-fluorophenyl)-3-phenylbut-2-en-1-ylidene)methanesulfonamide $\mathbf{1 h}$<smiles>CN=C(/C=C(\C)c1ccccc1)c1cccc(F)c1</smiles>

Yellow solid; M. P. $139-141{ }^{\circ} \mathrm{C},{ }^{1} \mathrm{H}$ NMR $\left(400 \mathrm{MHz}, \mathrm{CDCl}_{3}\right), \delta(\mathrm{ppm}) 7.72(\mathrm{~m}$, 1H), 7.66 (m, 1H), 7.62-7.55 (m, 2H), 7.49-7.34 (m, 4H), 7.33-7.24 (m, 1H), 7.06-7.00 $(\mathrm{m}, 1 \mathrm{H}), 3.25$ (s, 3H), 1.98-1.84 (m, 3H); ${ }^{13} \mathrm{C} \mathrm{NMR}\left(100 \mathrm{MHz}, \mathrm{CDCl}_{3}\right), \delta(\mathrm{ppm})$ 177.24, $162.19(\mathrm{~d}, J=246 \mathrm{~Hz}), 146.65,140.66,139.14(\mathrm{~d}, J=7 \mathrm{~Hz}), 130.53(\mathrm{~d}, J=8 \mathrm{~Hz})$, 129.06, 128.66, 126.37, $125.66(\mathrm{~d}, J=4 \mathrm{~Hz}), 120.80,120.39$ (d, $J=22 \mathrm{~Hz}), 115.95$ (d, $J=23 \mathrm{~Hz}), 42.90$, 19.68. ESI-HRMS calculated for $\mathrm{C}_{17} \mathrm{H}_{16} \mathrm{FNO}_{2} \mathrm{SNa}^{+}\left([\mathrm{M}+\mathrm{Na}]^{+}\right)$: 
340.0778; Found: 340.0775.

$\mathrm{N}-(E)-1$-(3-methoxyphenyl)-3-phenylbut-2-en-1-ylidene)methanesulfonamide 1i<smiles>C/N=C(\C=C(C)c1ccccc1)c1cccc(OC)c1</smiles>

Yellow solid; M. P. $106-108{ }^{\circ} \mathrm{C},{ }^{1} \mathrm{H}$ NMR $\left(400 \mathrm{MHz}, \mathrm{CDCl}_{3}\right), \delta(\mathrm{ppm}) 7.58$ (m, 2H), $7.54-7.46(\mathrm{~m}, 2 \mathrm{H}), 7.44-7.33(\mathrm{~m}, 4 \mathrm{H}), 7.17$ - $7.10(\mathrm{~m}, 1 \mathrm{H}), 7.05(\mathrm{~s}, 1 \mathrm{H}), 3.86(\mathrm{~s}$, 3H), 3.24 (s, 3H), 1.91 (s, 3H); ${ }^{13} \mathrm{C} \mathrm{NMR}\left(100 \mathrm{MHz}, \mathrm{CDCl}_{3}\right), \delta$ (ppm) 178.49, 159.92, $146.18,140.93,138.33,129.90,128.89,128.61,126.37,122.63,121.38,119.47,113.85$, 55.52, 42.91, 19.61. ESI-HRMS calculated for $\mathrm{C}_{18} \mathrm{H}_{19} \mathrm{NO}_{3} \mathrm{SNa}^{+}\left([\mathrm{M}+\mathrm{Na}]^{+}\right)$: 352.0978; Found: 352.0976.

$\mathrm{N}-(E)-1-([1,1$ '-biphenyl]-2-yl)-3-phenylbut-2-en-1-ylidene)methanesulfonamide $\mathbf{1 j}$<smiles>CN=C(/C=C(\C)c1ccccc1)c1ccccc1-c1ccccc1</smiles>

White solid; M. P. 103-105 ${ }^{\circ} \mathrm{C},{ }^{1} \mathrm{H}$ NMR (400 MHz, $\left.\mathrm{CDCl}_{3}\right), \delta$ (ppm) 8.03 (d, $J=$ $8.1 \mathrm{~Hz}, 2 \mathrm{H}), 7.73-7.67(\mathrm{~m}, 2 \mathrm{H}), 7.67-7.58(\mathrm{~m}, 4 \mathrm{H}), 7.49(\mathrm{t}, J=7.4 \mathrm{~Hz}, 2 \mathrm{H}), 7.45-$ $7.34(\mathrm{~m}, 4 \mathrm{H}), 7.08(\mathrm{~s}, 1 \mathrm{H}), 3.26$ (s, 3H), $1.96(\mathrm{~s}, 3 \mathrm{H}) ;{ }^{13} \mathrm{C} \mathrm{NMR}\left(100 \mathrm{MHz}, \mathrm{CDCl}_{3}\right), \delta$ (ppm) 178.18, 146.23, 145.78, 140.91, 139.66, 135.59, 130.28, 129.06, 128.88, 128.63, 128.46, 127.52, 127.29, 126.38, 121.34, 42.93, 19.66. ESI-HRMS calculated for $\mathrm{C}_{23} \mathrm{H}_{21} \mathrm{NO}_{2} \mathrm{SNa}^{+}\left([\mathrm{M}+\mathrm{Na}]^{+}\right)$: 398.1185; Found: 398.1180 .

N-(E)-1-phenyl-3-(p-tolyl)but-2-en-1-ylidene)methanesulfonamide 1k<smiles>C/C(=C/C(=N)c1ccccc1)c1ccc(C)cc1</smiles>

Yellow solid; M. P. $119-121{ }^{\circ} \mathrm{C},{ }^{1} \mathrm{H}$ NMR $\left(400 \mathrm{MHz}, \mathrm{CDCl}_{3}\right), \delta(\mathrm{ppm}) 7.93(\mathrm{~d}, J$ $=7.7 \mathrm{~Hz}, 2 \mathrm{H}), 7.58(\mathrm{~m}, 1 \mathrm{H}), 7.47(\mathrm{~m}, 4 \mathrm{H}), 7.20(\mathrm{~d}, J=7.9 \mathrm{~Hz}, 2 \mathrm{H}), 7.08(\mathrm{~s}, 1 \mathrm{H}), 3.23$ (s, 3H), 2.38 (s, 3H), 1.87 (s, 3H); $\left.{ }^{13} \mathrm{C} \mathrm{NMR} \mathrm{(100} \mathrm{MHz,} \mathrm{CDCl}_{3}\right), \delta(\mathrm{ppm}) 178.76,146.52$, 
139.06, 138.01, 137.20, 133.32, 129.66, 129.29, 128.88, 126.28, 120.53, 42.91, 21.26, 19.66. ESI-HRMS calculated for $\mathrm{C}_{18} \mathrm{H}_{20} \mathrm{NO}_{2} \mathrm{~S}^{+}\left([\mathrm{M}+\mathrm{H}]^{+}\right)$: 314.1202 ; Found: 314.1209 .

N-(E)-3-(4-chlorophenyl)-1-phenylbut-2-en-1 ylidene)methanesulfonamide $\mathbf{1 l}$<smiles>C/C(=C\C(c1ccccc1)=[N+](C)C)c1ccc(Cl)cc1</smiles>

Yellow solid; M. P. $126-128{ }^{\circ} \mathrm{C},{ }^{1} \mathrm{H}$ NMR $\left(400 \mathrm{MHz}, \mathrm{CDCl}_{3}\right), \delta(\mathrm{ppm}) 7.94(\mathrm{~d}, J$ $=7.7 \mathrm{~Hz}, 2 \mathrm{H}), 7.60(\mathrm{~m}, 1 \mathrm{H}), 7.55-7.44(\mathrm{~m}, 4 \mathrm{H}), 7.41-7.33(\mathrm{~m}, 2 \mathrm{H}), 7.01(\mathrm{~s}, 1 \mathrm{H}), 3.25$

(s, 3H), 1.88 (s, 3H); ${ }^{13} \mathrm{C}$ NMR (100 MHz, $\left.\mathrm{CDCl}_{3}\right), \delta$ (ppm) 178.23, 144.42, 139.34, $136.62,134.77,133.59,129.62,128.96,128.76,127.68,121.71,42.90,19.46$. ESIHRMS calculated for $\mathrm{C}_{17} \mathrm{H}_{17} \mathrm{ClNO}_{2} \mathrm{~S}^{+}\left([\mathrm{M}+\mathrm{H}]^{+}\right)$: 334.0658 ; Found: 334.0663 .

$\mathrm{N}$-(E)-3-(naphthalen-2-yl)-1-phenylbut-2-en-1-ylidene)methanesulfonamide $\mathbf{1 m}$<smiles>CN=C(/C=C(\C)c1ccc2ccccc2c1)c1ccccc1</smiles>

Brown solid; M. P. $135-137{ }^{\circ} \mathrm{C},{ }^{1} \mathrm{H}$ NMR (400 MHz, $\left.\mathrm{CDCl}_{3}\right), \delta(\mathrm{ppm})$ 8.05-7.94 (m, 3H), $7.86(\mathrm{~m}, 3 \mathrm{H}), 7.75(\mathrm{dd}, J=8.7,2.0 \mathrm{~Hz}, 1 \mathrm{H}), 7.61$ (t, $J=7.4 \mathrm{~Hz}, 1 \mathrm{H}), 7.55-$ $7.44(\mathrm{~m}, 4 \mathrm{H}), 7.22(\mathrm{~s}, 1 \mathrm{H}), 3.26(\mathrm{~s}, 3 \mathrm{H}), 2.01(\mathrm{~s}, 3 \mathrm{H}) ;{ }^{13} \mathrm{C} \mathrm{NMR}\left(100 \mathrm{MHz}, \mathrm{CDCl}_{3}\right), \delta$ (ppm) 178.59, 145.96, 138.08, 136.98, 133.46, 133.18, 129.69, 128.94, 128.54, 128.29, 127.63, 126.62, 126.48, 125.77, 124.09, 121.80, 42.92, 19.69. ESI-HRMS calculated for $\mathrm{C}_{21} \mathrm{H}_{20} \mathrm{NO}_{2} \mathrm{~S}^{+}\left([\mathrm{M}+\mathrm{H}]^{+}\right)$: 350.1204; Found: 350.1209 .

$\mathrm{N}-(E)$-3-(furan-2-yl)-1-phenylbut-2-en-1-ylidene)methanesulfonamide 1n<smiles>CN=C(/C=C(\C)c1ccco1)c1ccccc1</smiles>

Red solid; M. P. 98-100 ${ }^{\circ} \mathrm{C},{ }^{1} \mathrm{H}$ NMR $\left(400 \mathrm{MHz}, \mathrm{CDCl}_{3}\right), \delta(\mathrm{ppm}) 7.85(\mathrm{~d}, J=7.8$ $\mathrm{Hz}, 2 \mathrm{H}), 7.57(\mathrm{~m}, 1 \mathrm{H}), 7.50(\mathrm{~d}, J=1.8 \mathrm{~Hz}, 1 \mathrm{H}), 7.49$ - $7.36(\mathrm{~m}, 3 \mathrm{H}), 6.67$ - $6.52(\mathrm{~m}$, 
1H), $6.46(\mathrm{dd}, J=3.5,1.8 \mathrm{~Hz}, 1 \mathrm{H}), 3.23(\mathrm{~s}, 3 \mathrm{H}), 1.74(\mathrm{~s}, 3 \mathrm{H}) ;{ }^{13} \mathrm{C}$ NMR (100 MHz, $\left.\mathrm{CDCl}_{3}\right), \delta$ (ppm) 177.78, 153.79, 144.22, 137.84, 136.25, 133.08, 129.61, 128.81, 117.65, 112.02, 110.97, 42.99, 17.08. ESI-HRMS calculated for $\mathrm{C}_{15} \mathrm{H}_{16} \mathrm{NO}_{3} \mathrm{~S}^{+}$ $\left([\mathrm{M}+\mathrm{H}]^{+}\right): 290.0841$; Found: 290.0845 .

N-(3-methyl-1-phenylbut-2-en-1-ylidene)methanesulfonamide 10<smiles></smiles>

Yellow solid; M. P. $102-104{ }^{\circ} \mathrm{C},{ }^{1} \mathrm{H}$ NMR (400 MHz, $\left.\mathrm{CDCl}_{3}\right), \delta(\mathrm{ppm}) 7.83(\mathrm{~d}, J$ $=7.7 \mathrm{~Hz}, 2 \mathrm{H}), 7.56(\mathrm{~m}, 1 \mathrm{H}), 7.44(\mathrm{~m}, 2 \mathrm{H}), 6.63(\mathrm{~s}, 1 \mathrm{H}), 3.20(\mathrm{~s}, 3 \mathrm{H}), 2.01(\mathrm{~d}, J=1.3$ $\mathrm{Hz}, 3 \mathrm{H}), 1.47$ (s, 3H); ${ }^{13} \mathrm{C}$ NMR (100 MHz, $\left.\mathrm{CDCl}_{3}\right), \delta(\mathrm{ppm}) 178.60,148.81,137.64$, 132.99, 129.62, 128.73, 120.40, 43.03, 26.64, 22.13. ESI-HRMS calculated for $\mathrm{C}_{12} \mathrm{H}_{16} \mathrm{NO}_{2} \mathrm{~S}^{+}\left([\mathrm{M}+\mathrm{H}]^{+}\right): 238.0894$; Found: 238.0896 .

$\mathrm{N}-(E)$-1,3-diphenylallylidene)methanesulfonamide $\mathbf{1 p}$<smiles>CN(C)C(/C=C/c1ccccc1)c1ccccc1</smiles>

White solid; M. P. $97-99{ }^{\circ} \mathrm{C},{ }^{1} \mathrm{H}$ NMR $\left(400 \mathrm{MHz}, \mathrm{CDCl}_{3}\right), \delta(\mathrm{ppm}) 7.94(\mathrm{~d}, J=$ $16.1 \mathrm{~Hz}, 1 \mathrm{H}), 7.70(\mathrm{~d}, J=7.6 \mathrm{~Hz}, 2 \mathrm{H}), 7.57(\mathrm{~m}, 3 \mathrm{H}), 7.49(\mathrm{~m}, 2 \mathrm{H}), 7.45-7.36(\mathrm{~m}, 3 \mathrm{H})$, $7.08(\mathrm{~d}, J=16.0 \mathrm{~Hz}, 1 \mathrm{H}), 3.25(\mathrm{~s}, 3 \mathrm{H}) ;{ }^{13} \mathrm{C} \mathrm{NMR}\left(100 \mathrm{MHz}, \mathrm{CDCl}_{3}\right), \delta(\mathrm{ppm}) 177.98$, 149.23, 137.09, 134.40, 132.08, 131.25, 130.13, 129.06, 128.87, 128.54, 122.34, 43.27. ESI-HRMS calculated for $\mathrm{C}_{16} \mathrm{H}_{16} \mathrm{NO}_{2} \mathrm{~S}^{+}\left([\mathrm{M}+\mathrm{H}]^{+}\right)$: 286.0889; Found: 286.0896 .

$\mathrm{N}-((E)-1,3$-diphenylallylidene)-4-methylbenzenesulfonamide $\mathbf{1 q}$<smiles>[N+]=C(/C=C/c1ccccc1)c1ccccc1</smiles> 
Yellow solid; M. P. $116-118{ }^{\circ} \mathrm{C},{ }^{1} \mathrm{H}$ NMR (400 MHz, $\left.\mathrm{CDCl}_{3}\right), \delta(\mathrm{ppm}) 8.09$ (s, 1H), $7.93(\mathrm{~d}, J=7.7 \mathrm{~Hz}, 2 \mathrm{H}), 7.65(\mathrm{~d}, J=7.5 \mathrm{~Hz}, 2 \mathrm{H}), 7.61-7.50(\mathrm{~m}, 3 \mathrm{H}), 7.49-7.36$ $(\mathrm{m}, 5 \mathrm{H}), 7.31(\mathrm{~d}, J=8.0 \mathrm{~Hz}, 2 \mathrm{H}), 7.07(\mathrm{~d}, J=16.1 \mathrm{~Hz}, 1 \mathrm{H}), 2.42(\mathrm{~s}, 3 \mathrm{H}) ;{ }^{13} \mathrm{C} \mathrm{NMR}$ (100 MHz, $\left.\mathrm{CDCl}_{3}\right), \delta$ (ppm) 148.92, 143.50, 138.71, 134.57, 131.14, 130.26, 129.48, $129.07,128.78,128.39,127.23,21.62$. The characterization data of compound $\mathbf{1 q}$ is in accordance with the reported data in the literature. ${ }^{[17]}$

\section{General procedure for asymmetric hydrogenation of $\alpha, \beta$-unsaturated}

\section{ketoimines}

A stock solution was made by mixing $\mathrm{Ni}(\mathrm{OAc})_{2}(8.8 \mathrm{mg}, 0.05 \mathrm{mmol})$ with $(S, S)$ Ph-BPE (27.9 mg, $0.055 \mathrm{mmol})$ in a 1:1.1 molar ratio in anhydrous $\mathrm{MeOH}(1 \mathrm{~mL})$ at room temperature overnight in a nitrogen-filled glovebox. An aliquot of the catalyst solution $(0.1 \mathrm{~mL}, 0.005 \mathrm{mmol})$ was transferred by syringe into the vials charged with different substrates $(0.1 \mathrm{mmol}$ for each). The vials were subsequently transferred into an autoclave into which hydrogen gas was charged. The reaction was then stirred under $\mathrm{H}_{2}(80 \mathrm{~atm})$ at room temperature for $24 \mathrm{~h}$. The hydrogen gas was released slowly and carefully. The solution was concentrated and passed through a short column of silica gel (eluent: EtOAc) to remove the metal complex. The ee values of all compounds 2 were determined by HPLC analysis on a chiral stationary phase.

(E)-N-(1,3-diphenyl-1 $\lambda^{3}$-but-2-en-1-yl)methanesulfonamide 2a<smiles>C/C(=C\[C@@H](c1ccccc1)N(C)C)c1ccccc1</smiles>

The title compound was purified by flash column chromatography using EA to provide desired hydrogenation product; White solid; $29.9 \mathrm{mg}, 99 \%$ yield; $98 \%$ ee; $[\alpha]_{\mathrm{D}}{ }^{20}$ $=-5.3\left(\mathrm{c}=1.0, \mathrm{CHCl}_{3}\right)$; The enantiomeric excess was determined by HPLC on chiralpak AD-H column, hexane: isopropanol $=90: 10$; flow rate $=1.0 \mathrm{~mL} / \mathrm{min}$; $\mathrm{UV}$ detection at $254 \mathrm{~nm} ; \mathrm{t}_{\mathrm{R}}=14.0 \mathrm{~min}$ (minor), $\mathrm{t}_{\mathrm{R}}=15.7 \mathrm{~min}$ (major). ${ }^{1} \mathrm{H} \mathrm{NMR}\left(400 \mathrm{MHz}, \mathrm{CDCl}_{3}\right), \delta$ (ppm) 7.44 (d, $J=7.0 \mathrm{~Hz}, 2 \mathrm{H}), 7.38$ (m, 4H), 7.36 - 7.27 (m, 4H), 5.89 (dd, $J=9.1,1.6$ 
$\mathrm{Hz}, 1 \mathrm{H}), 5.51(\mathrm{dd}, J=9.1,6.2 \mathrm{~Hz}, 1 \mathrm{H}), 4.83(\mathrm{~d}, J=6.1 \mathrm{~Hz}, 1 \mathrm{H}), 2.76(\mathrm{~s}, 3 \mathrm{H}), 2.22(\mathrm{~d}$, $J=1.4 \mathrm{~Hz}, 3 \mathrm{H}) ;{ }^{13} \mathrm{C} \mathrm{NMR}\left(100 \mathrm{MHz}, \mathrm{CDCl}_{3}\right), \delta$ (ppm) 142.31, 140.44, 139.07, 129.09, $128.46,128.15,127.80,127.03,126.76,125.92,56.14,42.33,16.60$. ESI-HRMS calculated for $\mathrm{C}_{17} \mathrm{H}_{19} \mathrm{NO}_{2} \mathrm{SNa}^{+}\left([\mathrm{M}+\mathrm{Na}]^{+}\right)$: 324.1029; Found: 324.1022 .

(E)-N-(1,3-diphenyl-1 $\lambda^{3}$-but-2-en-1-yl)-N,N-dimethyl-methanesulfonamide $\mathbf{2 b}$<smiles>CC(=O)N[C@H](/C=C(\C)c1ccccc1)c1ccccc1</smiles>

The title compound was purified by flash column chromatography using EA to provide desired hydrogenation product; White solid; $32.0 \mathrm{mg}, 97 \%$ yield; $97 \%$ ee; $[\alpha]_{\mathrm{D}}{ }^{20}$ $=-13.5\left(\mathrm{c}=1.0, \mathrm{CHCl}_{3}\right)$; The enantiomeric excess was determined by HPLC on chiralpak OD-H column, hexane: isopropanol $=95: 5$; flow rate $=1.0 \mathrm{~mL} / \mathrm{min}$; UV detection at $254 \mathrm{~nm} ; \mathrm{t}_{\mathrm{R}}=23.1 \mathrm{~min}$ (minor), $\mathrm{t}_{\mathrm{R}}=25.6 \mathrm{~min}$ (major). ${ }^{1} \mathrm{H} \mathrm{NMR}(400 \mathrm{MHz}$, $\left.\mathrm{CDCl}_{3}\right), \delta(\mathrm{ppm}) 7.45$ - $7.40(\mathrm{~m}, 2 \mathrm{H}), 7.38(\mathrm{~m}, 4 \mathrm{H}), 7.31(\mathrm{~m}, 4 \mathrm{H}), 5.88(\mathrm{~m}, 1 \mathrm{H}), 5.36$ $(\mathrm{dd}, J=9.2,5.5 \mathrm{~Hz}, 1 \mathrm{H}), 4.67(\mathrm{~d}, J=5.6 \mathrm{~Hz}, 1 \mathrm{H}), 2.63(\mathrm{~s}, 6 \mathrm{H}), 2.19$ (d, $J=1.4 \mathrm{~Hz}$, $3 \mathrm{H}) ;{ }^{13} \mathrm{C} \mathrm{NMR}\left(100 \mathrm{MHz}, \mathrm{CDCl}_{3}\right), \delta$ (ppm) 142.56, 141.34, 138.02, 128.88, 128.40, $127.89,127.61,127.59,127.02,125.93,56.44,37.62,16.54$. ESI-HRMS calculated for $\mathrm{C}_{18} \mathrm{H}_{22} \mathrm{~N}_{2} \mathrm{O}_{2} \mathrm{SNa}^{+}\left([\mathrm{M}+\mathrm{Na}]^{+}\right)$: 353.1294; Found: 353.1289.

(E)-N-(1,3-diphenyl-1 $\lambda^{3}$-but-2-en-1-yl)-4-methylbenzenesulfonamide 2c<smiles>C/C(=C\[C@H]([NH3+])c1ccccc1)c1ccccc1</smiles>

The title compound was purified by flash column chromatography using EA to provide desired hydrogenation product; White solid; $37.5 \mathrm{mg}$, 99\% yield; $97 \%$ ee; $[\alpha]_{\mathrm{D}}{ }^{20}=-35.4\left(\mathrm{c}=1.0, \mathrm{CHCl}_{3}\right)$; The enantiomeric excess was determined by HPLC on chiralpak AD-H column, hexane: isopropanol = 90:10; flow rate $=1.0 \mathrm{~mL} / \mathrm{min}$; UV detection at $254 \mathrm{~nm} ; \mathrm{t}_{\mathrm{R}}=19.7 \mathrm{~min}$ (major), $\mathrm{t}_{\mathrm{R}}=22.5 \mathrm{~min}$ (minor). ${ }^{1} \mathrm{H} \mathrm{NMR}(400 \mathrm{MHz}$, $\left.\mathrm{CDCl}_{3}\right), \delta(\mathrm{ppm}) 7.67(\mathrm{~d}, J=8.3 \mathrm{~Hz}, 2 \mathrm{H}), 7.32-7.21(\mathrm{~m}, 8 \mathrm{H}), 7.17(\mathrm{~d}, J=8.0 \mathrm{~Hz}, 2 \mathrm{H})$, $7.10(\mathrm{dd}, J=7.6,2.0 \mathrm{~Hz}, 2 \mathrm{H}), 5.58(\mathrm{dd}, J=9.1,1.5 \mathrm{~Hz}, 1 \mathrm{H}), 5.35(\mathrm{dd}, J=9.2,6.6 \mathrm{~Hz}$, 
1H), $4.95(\mathrm{~d}, J=6.6 \mathrm{~Hz}, 1 \mathrm{H}), 2.34(\mathrm{~s}, 3 \mathrm{H}), 1.97(\mathrm{~d}, J=1.4 \mathrm{~Hz}, 3 \mathrm{H}) ;{ }^{13} \mathrm{C} \mathrm{NMR}(100$ $\mathrm{MHz}, \mathrm{CDCl}_{3}$ ), $\delta$ (ppm) 143.29, 142.36, 140.13, 137.95, 137.90, 129.47, 128.76, 128.15, $127.79,127.46,127.28,126.93,126.89,125.83,56.08,21.49,16.31$. ESI-HRMS calculated for $\mathrm{C}_{23} \mathrm{H}_{23} \mathrm{NO}_{2} \mathrm{SNa}^{+}\left([\mathrm{M}+\mathrm{Na}]^{+}\right)$: 400.1342; Found: 400.1337 .

(E)-N-(1-(4-fluorophenyl)-3-phenyl-1 $\lambda^{3}$-but-2-en-1-yl)methanesulfonamide 2d<smiles>C/C(=C\[C@H](N)c1ccc(F)cc1)c1ccccc1</smiles>

The title compound was purified by flash column chromatography using EA to provide desired hydrogenation product; White solid; $31.7 \mathrm{mg}$, 99\% yield; 98\% ee; $[\alpha]_{\mathrm{D}}^{20}=-7.5\left(\mathrm{c}=1.0, \mathrm{CHCl}_{3}\right)$; The enantiomeric excess was determined by HPLC on chiralpak AD-H column, hexane: isopropanol = 90:10; flow rate $=1.0 \mathrm{~mL} / \mathrm{min}$; UV detection at $254 \mathrm{~nm} ; \mathrm{t}_{\mathrm{R}}=12.3 \mathrm{~min}\left(\right.$ minor), $\mathrm{t}_{\mathrm{R}}=17.4 \mathrm{~min}$ (major). ${ }^{1} \mathrm{H} \mathrm{NMR}(400 \mathrm{MHz}$, $\left.\mathrm{CDCl}_{3}\right), \delta(\mathrm{ppm}) 7.45-7.26(\mathrm{~m}, 7 \mathrm{H}), 7.10$ - $7.02(\mathrm{~m}, 2 \mathrm{H}), 5.86(\mathrm{dd}, J=9.1,1.4 \mathrm{~Hz}, 1 \mathrm{H})$, $5.49(\mathrm{dd}, J=9.2,6.4 \mathrm{~Hz}, 1 \mathrm{H}), 5.00(\mathrm{~d}, J=6.4 \mathrm{~Hz}, 1 \mathrm{H}), 2.77$ (s, 3H), 2.20 (d, $J=1.4$ $\mathrm{Hz}, 3 \mathrm{H}) ;{ }^{13} \mathrm{C} \mathrm{NMR}\left(100 \mathrm{MHz}, \mathrm{CDCl}_{3}\right), \delta(\mathrm{ppm}) 162.29$ (d, $\left.J=246 \mathrm{~Hz}\right), 142.07,139.10$, $136.23(\mathrm{~d}, J=3 \mathrm{~Hz}), 128.68(\mathrm{~d}, J=8 \mathrm{~Hz}), 128.43,127.83,126.42,125.83,115.86$ (d, $J=22 \mathrm{~Hz})$, 55.37, 42.22, 16.51. ESI-HRMS calculated for $\mathrm{C}_{17} \mathrm{H}_{18} \mathrm{FNO}_{2} \mathrm{SNa}^{+}$ $\left([\mathrm{M}+\mathrm{Na}]^{+}\right): 342.0934 ;$ Found: 342.0929 .

(E)-N-(1-(4-chlorophenyl)-3-phenyl-1 $\lambda^{3}$-but-2-en-1-yl)methanesulfonamide $2 \mathbf{e}$<smiles>C/C(=C\[C@H](NC(C)(C)C)c1ccc(Cl)cc1)c1ccccc1</smiles>

The title compound was purified by flash column chromatography using EA to provide desired hydrogenation product; White solid; $32.1 \mathrm{mg}$, 96\% yield; 99\% ee; $[\alpha]_{\mathrm{D}}{ }^{20}=10.7(\mathrm{c}=1.0, \mathrm{MeOH})$; The enantiomeric excess was determined by HPLC on chiralpak AD-H column, hexane: isopropanol = 90:10; flow rate $=1.0 \mathrm{~mL} / \mathrm{min}$; UV detection at $254 \mathrm{~nm} ; \mathrm{t}_{\mathrm{R}}=13.0 \mathrm{~min}$ (minor), $\mathrm{t}_{\mathrm{R}}=23.7 \mathrm{~min}$ (major). ${ }^{1} \mathrm{H} \mathrm{NMR}(400 \mathrm{MHz}$, $\left.\mathrm{CDCl}_{3}\right), \delta(\mathrm{ppm}) 7.41-7.26(\mathrm{~m}, 9 \mathrm{H}), 5.83(\mathrm{~m}, 1 \mathrm{H}), 5.47(\mathrm{dd}, J=9.2,6.5 \mathrm{~Hz}, 1 \mathrm{H}), 5.08$ 
$(\mathrm{d}, J=6.5 \mathrm{~Hz}, 1 \mathrm{H}), 2.77(\mathrm{~s}, 3 \mathrm{H}), 2.20(\mathrm{~d}, J=1.4 \mathrm{~Hz}, 3 \mathrm{H}) ;{ }^{13} \mathrm{C} \mathrm{NMR}\left(100 \mathrm{MHz}, \mathrm{CDCl}_{3}\right)$, $\delta(\mathrm{ppm}) 142.07,139.36,139.07,133.90,129.19,128.51,128.46,128.41,127.95$, 126.23, 125.91, 55.50, 42.31, 16.62. ESI-HRMS calculated for $\mathrm{C}_{17} \mathrm{H}_{18} \mathrm{ClNO}_{2} \mathrm{SNa}^{+}$ $\left([\mathrm{M}+\mathrm{Na}]^{+}\right)$: 358.0639; Found: 358.0631 .

(E)-N-(1-(4-methoxyphenyl)-3-phenyl-1 $\lambda^{3}$-but-2-en-1-yl)methanesulfonamide $\mathbf{2 f}$<smiles>COc1ccc([C@H](N)/C=C(\C)c2ccccc2)cc1</smiles>

The title compound was purified by flash column chromatography using EA to provide desired hydrogenation product; White solid; $32.7 \mathrm{mg}$, 99\% yield; >99\% ee; $[\alpha]_{\mathrm{D}}^{20}=10.4\left(\mathrm{c}=1.0, \mathrm{CHCl}_{3}\right)$; The enantiomeric excess was determined by HPLC on chiralpak AD-H column, hexane: isopropanol $=90: 10$; flow rate $=1.0 \mathrm{~mL} / \mathrm{min} ; \mathrm{UV}$ detection at $254 \mathrm{~nm} ; \mathrm{t}_{\mathrm{R}}=20.8 \mathrm{~min}$ (minor), $\mathrm{t}_{\mathrm{R}}=30.5 \mathrm{~min}$ (major). ${ }^{1} \mathrm{H} \mathrm{NMR}(400 \mathrm{MHz}$, $\left.\mathrm{CDCl}_{3}\right), \delta(\mathrm{ppm}) 7.41-7.27(\mathrm{~m}, 7 \mathrm{H}), 6.94-6.87(\mathrm{~m}, 2 \mathrm{H}), 5.89(\mathrm{~m}, 1 \mathrm{H}), 5.46(\mathrm{dd}, J=$ 9.1, $6.0 \mathrm{~Hz}, 1 \mathrm{H}), 4.74(\mathrm{~d}, J=6.0 \mathrm{~Hz}, 1 \mathrm{H}), 3.81(\mathrm{~s}, 3 \mathrm{H}), 2.75(\mathrm{~s}, 3 \mathrm{H}), 2.20$ (d, $J=1.4$ $\mathrm{Hz}, 3 \mathrm{H}) ;{ }^{13} \mathrm{C}$ NMR $\left(100 \mathrm{MHz}, \mathrm{CDCl}_{3}\right), \delta$ (ppm) 159.36, 142.38, 138.74, 132.42, 128.44 , $128.29,127.75,126.94,125.90,114.37,55.65,55.36,42.34,16.54$. ESI-HRMS calculated for $\mathrm{C}_{18} \mathrm{H}_{21} \mathrm{NO}_{3} \mathrm{SNa}^{+}\left([\mathrm{M}+\mathrm{Na}]^{+}\right)$: 354.1134; Found: 354.1130 .

(E)-N-(3-phenyl-1-(p-tolyl)-1 $\lambda^{3}$-but-2-en-1-yl)methanesulfonamide $\mathbf{2 g}$<smiles>C/C(=C\[C@@H](N)c1ccc(C)cc1)c1ccccc1</smiles>

The title compound was purified by flash column chromatography using EA to provide desired hydrogenation product; White solid; $31.1 \mathrm{mg}$, 99\% yield; $99 \%$ ee; $[\alpha]_{\mathrm{D}}^{20}=13.5(\mathrm{c}=1.0, \mathrm{MeOH})$; The enantiomeric excess was determined by HPLC on chiralpak AD-H column, hexane: isopropanol $=90: 10$; flow rate $=1.0 \mathrm{~mL} / \mathrm{min}$; UV detection at $254 \mathrm{~nm} ; \mathrm{t}_{\mathrm{R}}=14.5 \mathrm{~min}$ (minor), $\mathrm{t}_{\mathrm{R}}=22.4 \mathrm{~min}$ (major). ${ }^{1} \mathrm{H} \mathrm{NMR}(400 \mathrm{MHz}$, $\left.\mathrm{CDCl}_{3}\right), \delta(\mathrm{ppm}) 7.40-7.35(\mathrm{~m}, 2 \mathrm{H}), 7.35-7.26(\mathrm{~m}, 5 \mathrm{H}), 7.18(\mathrm{~d}, J=7.9 \mathrm{~Hz}, 2 \mathrm{H}), 5.89$ $(\mathrm{dd}, J=9.2,1.5 \mathrm{~Hz}, 1 \mathrm{H}), 5.47(\mathrm{~s}, 1 \mathrm{H}), 4.91(\mathrm{~d}, J=6.4 \mathrm{~Hz}, 1 \mathrm{H}), 2.75(\mathrm{~s}, 3 \mathrm{H}), 2.34$ (s, 
3H), $2.20(\mathrm{~d}, J=1.4 \mathrm{~Hz}, 3 \mathrm{H}) ;{ }^{13} \mathrm{C} \mathrm{NMR}\left(100 \mathrm{MHz}, \mathrm{CDCl}_{3}\right), \delta$ (ppm) 142.40, 138.69, 137.91, 137.49, 129.73, 128.43, 127.73, 126.98, 126.94, 125.92, 55.93, 42.30, 21.16, 16.55. ESI-HRMS calculated for $\mathrm{C}_{18} \mathrm{H}_{21} \mathrm{NO}_{2} \mathrm{SNa}^{+}\left([\mathrm{M}+\mathrm{Na}]^{+}\right)$: 338.1185; Found: 338.1184 .

(E)-N-(1-(3-fluorophenyl)-3-phenyl-1 $\lambda^{3}$-but-2-en-1-yl)methanesulfonamide $\mathbf{2 h}$<smiles>C/C(=C\[C@H](N)c1cccc(F)c1)c1ccccc1</smiles>

The title compound was purified by flash column chromatography using EA to provide desired hydrogenation product; White solid; $31.6 \mathrm{mg}, 99 \%$ yield; >99\% ee; $[\alpha]_{\mathrm{D}}^{20}=-13.8\left(\mathrm{c}=1.0, \mathrm{CHCl}_{3}\right)$; The enantiomeric excess was determined by HPLC on chiralpak AD-H column, hexane: isopropanol $=90: 10$; flow rate $=0.5 \mathrm{~mL} / \mathrm{min}$; UV detection at $254 \mathrm{~nm} ; \mathrm{t}_{\mathrm{R}}=23.2 \mathrm{~min}\left(\right.$ minor), $\mathrm{t}_{\mathrm{R}}=24.1 \mathrm{~min}$ (major). ${ }^{1} \mathrm{H} \mathrm{NMR}(400 \mathrm{MHz}$, $\left.\mathrm{CDCl}_{3}\right), \delta(\mathrm{ppm}) 7.41-7.27(\mathrm{~m}, 6 \mathrm{H}), 7.22(\mathrm{~d}, J=8.2 \mathrm{~Hz}, 1 \mathrm{H}), 7.15(\mathrm{~m}, 1 \mathrm{H}), 7.01(\mathrm{~m}$, 1H), $5.83(\mathrm{dd}, J=9.2,1.5 \mathrm{~Hz}, 1 \mathrm{H}), 5.52(\mathrm{~s}, 1 \mathrm{H}), 4.87$ (d, $J=6.3 \mathrm{~Hz}, 1 \mathrm{H}), 2.81(\mathrm{~s}, 3 \mathrm{H})$, $2.23(\mathrm{~d}, J=1.4 \mathrm{~Hz}, 3 \mathrm{H}) ;{ }^{13} \mathrm{C} \mathrm{NMR}\left(100 \mathrm{MHz}, \mathrm{CDCl}_{3}\right), \delta(\mathrm{ppm}) 163.02(\mathrm{~d}, J=246 \mathrm{~Hz})$, $143.06(\mathrm{~d}, J=7 \mathrm{~Hz}), 141.99,139.41,130.55$ (d, $J=8 \mathrm{~Hz}), 128.43,127.87,126.03$, 125.85, $122.55(\mathrm{~d}, J=3 \mathrm{~Hz}), 114.96(\mathrm{~d}, J=21 \mathrm{~Hz}), 113.88$ (d, $J=22 \mathrm{~Hz}), 55.54(\mathrm{~d}, J$ $=2 \mathrm{~Hz}), 42.22$, 16.55. ESI-HRMS calculated for $\mathrm{C}_{17} \mathrm{H}_{18} \mathrm{FNO}_{2} \mathrm{SNa}^{+}\left([\mathrm{M}+\mathrm{Na}]^{+}\right)$: 342.0934; Found: 342.0928.

(E)-N-(1-(3-methoxyphenyl)-3-phenyl-1 $\lambda^{3}$-but-2-en-1-yl)methanesulfonamide $2 \mathbf{i}$<smiles>COc1cccc([C@H](N)/C=C(\C)c2ccccc2)c1</smiles>

The title compound was purified by flash column chromatography using EA to provide desired hydrogenation product; White solid; $32.8 \mathrm{mg}$, 99\% yield; $99 \%$ ee; $[\alpha]_{\mathrm{D}}^{20}=4.6(\mathrm{c}=1.0, \mathrm{MeOH})$; The enantiomeric excess was determined by HPLC on chiralpak AD-H column, hexane: isopropanol = 90:10; flow rate $=1.0 \mathrm{~mL} / \mathrm{min}$; UV detection at $254 \mathrm{~nm} ; \mathrm{t}_{\mathrm{R}}=20.4 \mathrm{~min}$ (major), $\mathrm{t}_{\mathrm{R}}=22.6 \mathrm{~min}$ (minor). ${ }^{1} \mathrm{H} \mathrm{NMR}(400 \mathrm{MHz}$, 
$\left.\mathrm{CDCl}_{3}\right), \delta(\mathrm{ppm}) 7.41$ - $7.36(\mathrm{~m}, 2 \mathrm{H}), 7.36$ - $7.27(\mathrm{~m}, 4 \mathrm{H}), 7.05$ - $6.94(\mathrm{~m}, 2 \mathrm{H}), 6.85(\mathrm{dd}$, $J=8.2,2.6 \mathrm{~Hz}, 1 \mathrm{H}), 5.87(\mathrm{dd}, J=9.1,1.5 \mathrm{~Hz}, 1 \mathrm{H}), 5.47(\mathrm{dd}, J=9.1,6.2 \mathrm{~Hz}, 1 \mathrm{H}), 4.81$ $(\mathrm{d}, J=6.2 \mathrm{~Hz}, 1 \mathrm{H}), 3.82(\mathrm{~s}, 3 \mathrm{H}), 2.79(\mathrm{~s}, 3 \mathrm{H}), 2.22(\mathrm{~d}, J=1.4 \mathrm{~Hz}, 3 \mathrm{H}) ;{ }^{13} \mathrm{C}$ NMR $(100$ $\left.\mathrm{MHz}, \mathrm{CDCl}_{3}\right), \delta(\mathrm{ppm}) 160.08,142.30,142.07,139.14,130.16,128.45,127.80,126.67$, 125.92, 119.17, 113.29, 112.79, 56.09, 55.35, 42.34, 16.60. ESI-HRMS calculated for $\mathrm{C}_{18} \mathrm{H}_{21} \mathrm{NO}_{3} \mathrm{SNa}^{+}\left([\mathrm{M}+\mathrm{Na}]^{+}\right)$: 354.1134; Found: 354.1127 .

(E)-N-(1-([1,1'-biphenyl]-2-yl)-3-phenyl-1 $\lambda^{3}$-but-2-en-1-yl)methanesulfonamide $\mathbf{2 j}$<smiles>C/C(=C\[C@@H](c1ccccc1-c1ccccc1)N(C)C)c1ccccc1</smiles>

The title compound was purified by flash column chromatography using EA to provide desired hydrogenation product; White solid; $37.4 \mathrm{mg}, 99 \%$ yield; >99\% ee; $[\alpha]_{\mathrm{D}}^{20}=8.2\left(\mathrm{c}=1.0, \mathrm{CHCl}_{3}\right)$; The enantiomeric excess was determined by HPLC on chiralpak OD-H column, hexane: isopropanol $=85: 15$; flow rate $=1.0 \mathrm{~mL} / \mathrm{min}$; UV detection at $254 \mathrm{~nm} ; \mathrm{t}_{\mathrm{R}}=60.0 \mathrm{~min}$ (minor), $\mathrm{t}_{\mathrm{R}}=72.6 \mathrm{~min}$ (major). ${ }^{1} \mathrm{H} \mathrm{NMR}(400 \mathrm{MHz}$, $\left.\mathrm{CDCl}_{3}\right), \delta(\mathrm{ppm}) 7.63-7.53(\mathrm{~m}, 4 \mathrm{H}), 7.49(\mathrm{~d}, J=8.2 \mathrm{~Hz}, 2 \mathrm{H}), 7.47-7.38(\mathrm{~m}, 4 \mathrm{H}), 7.38$ - $7.27(\mathrm{~m}, 4 \mathrm{H}), 5.93(\mathrm{dd}, J=9.2,1.6 \mathrm{~Hz}, 1 \mathrm{H}), 5.55(\mathrm{dd}, J=9.2,6.5 \mathrm{~Hz}, 1 \mathrm{H}), 5.11$ (d, $J$ $=6.5 \mathrm{~Hz}, 1 \mathrm{H}), 2.79(\mathrm{~s}, 3 \mathrm{H}), 2.23(\mathrm{~d}, J=1.4 \mathrm{~Hz}, 3 \mathrm{H}) ;{ }^{13} \mathrm{C} \mathrm{NMR}\left(100 \mathrm{MHz}, \mathrm{CDCl}_{3}\right), \delta$ (ppm) 142.31, 140.98, 140.40, 139.49, 138.94, 128.91, 128.49, 127.83, 127.74, 127.59, 127.47, 127.11, 126.72, 125.96, 55.90, 42.34, 16.63. ESI-HRMS calculated for $\mathrm{C}_{23} \mathrm{H}_{23} \mathrm{NO}_{2} \mathrm{SNa}^{+}\left([\mathrm{M}+\mathrm{Na}]^{+}\right)$: 400.1342 ; Found: 400.1337.

(E)-N-(1-phenyl-3-(p-tolyl)- $1 \lambda^{3}$-but-2-en-1-yl)methanesulfonamide $2 \mathbf{k}$<smiles>C/C(=C\[C@H](NC(C)(C)C)c1ccccc1)c1ccc(C)cc1</smiles>

The title compound was purified by flash column chromatography using EA to provide desired hydrogenation product; White solid; $29.9 \mathrm{mg}$, 95\% yield; $97 \%$ ee; $[\alpha]_{\mathrm{D}}^{20}=-5.2\left(\mathrm{c}=1.0, \mathrm{CHCl}_{3}\right)$; The enantiomeric excess was determined by HPLC on chiralpak OD-H column, hexane: isopropanol $=85: 15$; flow rate $=1.0 \mathrm{~mL} / \mathrm{min}$; UV 
detection at $254 \mathrm{~nm} ; \mathrm{t}_{\mathrm{R}}=9.8 \mathrm{~min}$ (major), $\mathrm{t}_{\mathrm{R}}=30.6 \mathrm{~min}$ (minor). ${ }^{1} \mathrm{H} \mathrm{NMR}(400 \mathrm{MHz}$, $\left.\mathrm{CDCl}_{3}\right), \delta(\mathrm{ppm}) 7.43(\mathrm{~d}, J=7.5 \mathrm{~Hz}, 2 \mathrm{H}), 7.37(\mathrm{t}, J=7.5 \mathrm{~Hz}, 2 \mathrm{H}), 7.30(\mathrm{t}, J=8.4 \mathrm{~Hz}$, $3 \mathrm{H}), 7.13(\mathrm{~d}, J=7.8 \mathrm{~Hz}, 2 \mathrm{H}), 5.87(\mathrm{~d}, J=9.1 \mathrm{~Hz}, 1 \mathrm{H}), 5.49(\mathrm{dd}, J=9.2,6.4 \mathrm{~Hz}, 1 \mathrm{H})$, $4.98(\mathrm{~d}, J=6.4 \mathrm{~Hz}, 1 \mathrm{H}), 2.74$ (s, 3H), 2.34 (s, 3H), 2.19 (s, 3H); ${ }^{13} \mathrm{C}$ NMR (100 MHz, $\left.\mathrm{CDCl}_{3}\right), \delta(\mathrm{ppm}) 140.61,139.39,138.69,137.64,129.13,129.03,128.05,127.04$ 126.00, 125.78, 56.16, 42.24, 21.12, 16.53. ESI-HRMS calculated for $\mathrm{C}_{18} \mathrm{H}_{21} \mathrm{NO}_{2} \mathrm{SNa}^{+}$ $\left([\mathrm{M}+\mathrm{Na}]^{+}\right): 338.1179 ;$ Found: 338.1185 .

(E)-N-(3-(4-chlorophenyl)-1-phenyl-1 $\lambda^{3}$-but-2-en-1-yl)methanesulfonamide 21<smiles>C/C(=C\[C@H](N)c1ccccc1)c1ccc(Cl)cc1</smiles>

The title compound was purified by flash column chromatography using EA to provide desired hydrogenation product; White solid; $32.1 \mathrm{mg}, 96 \%$ yield; $97 \%$ ee; $[\alpha]_{\mathrm{D}}^{20}=0.4\left(\mathrm{c}=1.0, \mathrm{CHCl}_{3}\right)$; The enantiomeric excess was determined by HPLC on chiralpak OD-H column, hexane: isopropanol $=90: 10$; flow rate $=1.0 \mathrm{~mL} / \mathrm{min}$; UV detection at $254 \mathrm{~nm} ; \mathrm{t}_{\mathrm{R}}=14.6 \mathrm{~min}\left(\right.$ major), $\mathrm{t}_{\mathrm{R}}=28.2 \min \left(\right.$ minor). ${ }^{1} \mathrm{H} \mathrm{NMR}(400 \mathrm{MHz}$, $\left.\mathrm{CDCl}_{3}\right), \delta(\mathrm{ppm}) 7.47-7.35(\mathrm{~m}, 4 \mathrm{H}), 7.29(\mathrm{~d}, J=2.4 \mathrm{~Hz}, 5 \mathrm{H}), 5.89(\mathrm{~d}, J=9.1 \mathrm{~Hz}, 1 \mathrm{H})$, $5.48(\mathrm{dd}, J=9.1,6.3 \mathrm{~Hz}, 1 \mathrm{H}), 5.06(\mathrm{~d}, J=6.3 \mathrm{~Hz}, 1 \mathrm{H}), 2.73(\mathrm{~s}, 3 \mathrm{H}), 2.18(\mathrm{~s}, 3 \mathrm{H}) ;{ }^{13} \mathrm{C}$ NMR (100 MHz, $\mathrm{CDCl}_{3}$ ), $\delta$ (ppm) 140.71, 140.36, 137.68, 133.55, 129.14, 128.55, 128.20, 127.31, 127.23, 126.98, 56.16, 42.26, 16.52. ESI-HRMS calculated for $\mathrm{C}_{17} \mathrm{H}_{18} \mathrm{ClNO}_{2} \mathrm{SNa}^{+}\left([\mathrm{M}+\mathrm{Na}]^{+}\right): 358.0635$; Found: 358.0639 .

(E)-N-(3-(naphthalen-2-yl)-1-phenyl-1 $\lambda^{3}$-but-2-en-1-yl)methanesulfonamide $\mathbf{2 m}$<smiles>C/C(=C\[C@H](NC(C)(C)C)c1ccccc1)c1ccc2ccccc2c1</smiles>

The title compound was purified by flash column chromatography using EA to provide desired hydrogenation product; Brown solid; $32.5 \mathrm{mg}$, 92\% yield; $98 \%$ ee; $[\alpha]_{\mathrm{D}}^{20}=7.9\left(\mathrm{c}=1.0, \mathrm{CHCl}_{3}\right)$; The enantiomeric excess was determined by HPLC on chiralpak AD-H column, hexane: isopropanol = 90:10; flow rate $=1.0 \mathrm{~mL} / \mathrm{min}$; UV 
detection at $254 \mathrm{~nm} ; \mathrm{t}_{\mathrm{R}}=22.6 \mathrm{~min}$ (minor), $\mathrm{t}_{\mathrm{R}}=24.4 \mathrm{~min}$ (major). ${ }^{1} \mathrm{H} \mathrm{NMR}(400 \mathrm{MHz}$, $\left.\mathrm{CDCl}_{3}\right), \delta(\mathrm{ppm}) 7.84-7.75(\mathrm{~m}, 4 \mathrm{H}), 7.53(\mathrm{dd}, J=8.6,1.9 \mathrm{~Hz}, 1 \mathrm{H}), 7.50-7.42(\mathrm{~m}$, 4H), $7.42-7.35(\mathrm{~m}, 2 \mathrm{H}), 7.34-7.28(\mathrm{~m}, 1 \mathrm{H}), 6.03(\mathrm{dd}, J=9.1,1.4 \mathrm{~Hz}, 1 \mathrm{H}), 5.56(\mathrm{dd}$, $J=9.1,6.4 \mathrm{~Hz}, 1 \mathrm{H}), 5.02(\mathrm{~d}, J=6.4 \mathrm{~Hz}, 1 \mathrm{H}), 2.76(\mathrm{~s}, 3 \mathrm{H}), 2.31(\mathrm{~d}, J=1.4 \mathrm{~Hz}, 3 \mathrm{H})$; ${ }^{13} \mathrm{C} \mathrm{NMR}\left(100 \mathrm{MHz}, \mathrm{CDCl}_{3}\right), \delta(\mathrm{ppm}) 140.50,139.43,138.75,133.29,132.87,129.12$, $128.19,128.16,128.02,127.58,127.30,127.05,126.40,126.13,124.84,124.07,56.26$, 42.31, 16.61. ESI-HRMS calculated for $\mathrm{C}_{21} \mathrm{H}_{21} \mathrm{NO}_{2} \mathrm{SNa}^{+}\left([\mathrm{M}+\mathrm{Na}]^{+}\right)$: 374.1177; Found: 374.1185 .

(E)-N-(3-(furan-2-yl)-1-phenyl-1 $\lambda^{3}$-but-2-en-1-yl)methanesulfonamide 2n<smiles>C/C(=C\[C@@H](c1ccccc1)N(C)C)c1ccco1</smiles>

The title compound was purified by flash column chromatography using EA to provide desired hydrogenation product; Red solid; $25.7 \mathrm{mg}, 88 \%$ yield; $96 \%$ ee; $[\alpha]_{\mathrm{D}}{ }^{20}$ $=12.4\left(\mathrm{c}=0.5, \mathrm{CHCl}_{3}\right)$; The enantiomeric excess was determined by HPLC on chiralpak AD-H column, hexane: isopropanol $=90: 10 ;$ flow rate $=1.0 \mathrm{~mL} / \mathrm{min} ; \mathrm{UV}$ detection at $254 \mathrm{~nm} ; \mathrm{t}_{\mathrm{R}}=15.6 \mathrm{~min}\left(\right.$ minor), $\mathrm{t}_{\mathrm{R}}=22.1 \mathrm{~min}$ (major). ${ }^{1} \mathrm{H} \mathrm{NMR}(400 \mathrm{MHz}$, $\left.\mathrm{CDCl}_{3}\right), \delta(\mathrm{ppm}) 7.46$ - $7.40(\mathrm{~m}, 2 \mathrm{H}), 7.41$ - $7.33(\mathrm{~m}, 3 \mathrm{H}), 7.33$ - $7.28(\mathrm{~m}, 1 \mathrm{H}), 6.43$ $6.31(\mathrm{~m}, 2 \mathrm{H}), 6.18(\mathrm{~m}, 1 \mathrm{H}), 5.49(\mathrm{dd}, J=9.5,6.1 \mathrm{~Hz}, 1 \mathrm{H}), 4.83(\mathrm{dd}, J=6.4,2.9 \mathrm{~Hz}$, $1 \mathrm{H}), 2.78(\mathrm{~s}, 3 \mathrm{H}), 2.10(\mathrm{~d}, J=1.4 \mathrm{~Hz}, 3 \mathrm{H}) ;{ }^{13} \mathrm{C} \mathrm{NMR}\left(100 \mathrm{MHz}, \mathrm{CDCl}_{3}\right), \delta(\mathrm{ppm})$ 154.43, 142.27, 140.28, 129.08, 128.16, 127.81, 126.99, 126.94, 123.03, 111.40, 107.37, 55.57, 42.44, 13.78. ESI-HRMS calculated for $\mathrm{C}_{15} \mathrm{H}_{17} \mathrm{NO}_{3} \mathrm{SNa}^{+}\left([\mathrm{M}+\mathrm{Na}]^{+}\right): 314.0853$; Found: 314.0821.

N-(3-methyl-1-phenyl-1 $\lambda^{3}$-but-2-en-1-yl)methanesulfonamide 20<smiles>CC(C)=C[C@H](N)c1ccccc1</smiles> 
The title compound was purified by flash column chromatography using EA to provide desired hydrogenation product; Pale oil; $21.7 \mathrm{mg}, 91 \%$ yield; $>99 \%$ ee; $[\alpha]_{\mathrm{D}}{ }^{20}$ $=-23.2\left(\mathrm{c}=0.5, \mathrm{CHCl}_{3}\right)$; The enantiomeric excess was determined by HPLC on chiralpak AD-H column, hexane: isopropanol $=90: 10$; flow rate $=1.0 \mathrm{~mL} / \mathrm{min}$; UV detection at $220 \mathrm{~nm} ; \mathrm{t}_{\mathrm{R}}=9.2 \mathrm{~min}$ (major), $\mathrm{t}_{\mathrm{R}}=10.0 \mathrm{~min}$ (minor). ${ }^{1} \mathrm{H} \mathrm{NMR}(400 \mathrm{MHz}$, $\left.\mathrm{CDCl}_{3}\right), \delta(\mathrm{ppm}) 7.41-7.32(\mathrm{~m}, 4 \mathrm{H}), 7.30(\mathrm{~m}, 1 \mathrm{H}), 5.40-5.23(\mathrm{~m}, 2 \mathrm{H}), 4.76(\mathrm{~d}, J=$ $5.2 \mathrm{~Hz}, 1 \mathrm{H}), 2.71(\mathrm{~s}, 3 \mathrm{H}), 1.79(\mathrm{dd}, J=18.1,1.1 \mathrm{~Hz}, 6 \mathrm{H}) ;{ }^{13} \mathrm{C} \mathrm{NMR}\left(100 \mathrm{MHz}, \mathrm{CDCl}_{3}\right)$, $\delta$ (ppm) 141.04, 136.77, 129.01, 128.92, 127.86, 126.87, 126.64, 124.35, 55.84, 42.16, 25.77, 18.37. ESI-HRMS calculated for $\mathrm{C}_{12} \mathrm{H}_{17} \mathrm{NO}_{2} \mathrm{SNa}^{+}\left([\mathrm{M}+\mathrm{Na}]^{+}\right)$: 262.0866; Found: 262.0872 .

(E)-N-(1,3-diphenyl-1 $\lambda^{3}$-allyl)methanesulfonamide $\mathbf{2 p}$<smiles>CN(C)C(/C=C/c1ccccc1)c1ccccc1</smiles>

The title compound was purified by flash column chromatography using EA to provide desired hydrogenation product; White solid; $17.1 \mathrm{mg}, 60 \%$ yield; $96 \%$ ee; $[\alpha]_{\mathrm{D}}^{20}=-3.0\left(\mathrm{c}=0.5, \mathrm{CHCl}_{3}\right)$; The enantiomeric excess was determined by HPLC on chiralpak OD-H column, hexane: isopropanol $=90: 10$; flow rate $=1.0 \mathrm{~mL} / \mathrm{min}$; UV detection at $254 \mathrm{~nm} ; \mathrm{t}_{\mathrm{R}}=26.0 \mathrm{~min}$ (major), $\mathrm{t}_{\mathrm{R}}=44.9 \min ($ minor $) .{ }^{1} \mathrm{H} \mathrm{NMR}(400 \mathrm{MHz}$, $\left.\mathrm{CDCl}_{3}\right), \delta(\mathrm{ppm}) 7.39(\mathrm{dd}, J=5.6,4.1 \mathrm{~Hz}, 5 \mathrm{H}), 7.37$ - $7.26(\mathrm{~m}, 5 \mathrm{H}), 6.62(\mathrm{dd}, J=15.9$, $1.3 \mathrm{~Hz}, 1 \mathrm{H}), 6.33(\mathrm{dd}, J=15.8,6.8 \mathrm{~Hz}, 1 \mathrm{H}), 5.28(\mathrm{~m}, 1 \mathrm{H}), 5.19-5.01(\mathrm{~m}, 1 \mathrm{H}), 2.77$ (s, 3H); ${ }^{13} \mathrm{C}$ NMR (100 MHz, $\left.\mathrm{CDCl}_{3}\right), \delta$ (ppm) 139.84, 135.88, 132.51, 129.12, 129.10, $128.73,128.53,128.43,128.33,128.26,127.21,126.70,126.67,59.79,42.29$. ESIHRMS calculated for $\mathrm{C}_{16} \mathrm{H}_{17} \mathrm{NO}_{2} \mathrm{SNa}^{+}\left([\mathrm{M}+\mathrm{Na}]^{+}\right)$: 310.0875 ; Found: 310.0872 .

(E)-N-(1,3-diphenyl-1 $\lambda^{3}$-allyl)-4-methylbenzenesulfonamide $\mathbf{2 q}$<smiles>[NH3+]C(/C=C/c1ccccc1)c1ccccc1</smiles> 
The title compound was purified by flash column chromatography using EA to provide desired hydrogenation product; White solid; $19.6 \mathrm{mg}$, 54\% yield; $90 \%$ ee; $[\alpha]_{\mathrm{D}}^{20}=-20.6\left(\mathrm{c}=1.0, \mathrm{CHCl}_{3}\right),\left(\right.$ for $R-2 q$ in Ref. $18:\left([\alpha]_{\mathrm{D}}{ }^{25}=-31.4\left(\mathrm{c}=1.0, \mathrm{CHCl}_{3}\right)\right.$, 95\% ee for $R$ ); The enantiomeric excess was determined by HPLC on chiralpak AD-H column, hexane: isopropanol $=95: 5$; flow rate $=1.0 \mathrm{~mL} / \mathrm{min} ; \mathrm{UV}$ detection at $210 \mathrm{~nm}$; $\mathrm{t}_{\mathrm{R}}=48.9 \min ($ major $), \mathrm{t}_{\mathrm{R}}=56.4 \mathrm{~min}$ (minor). ${ }^{1} \mathrm{H} \mathrm{NMR}\left(400 \mathrm{MHz}, \mathrm{CDCl}_{3}\right), \delta(\mathrm{ppm}) 7.71$ - $7.61(\mathrm{~m}, 2 \mathrm{H}), 7.27-7.22(\mathrm{~m}, 5 \mathrm{H}), 7.21-7.17(\mathrm{~m}, 2 \mathrm{H}), 7.17-7.14(\mathrm{~m}, 2 \mathrm{H}), 7.13$ (d, $J=2.0 \mathrm{~Hz}, 1 \mathrm{H}), 6.41-6.28(\mathrm{~m}, 1 \mathrm{H}), 6.07(\mathrm{dd}, J=15.8,6.3 \mathrm{~Hz}, 1 \mathrm{H}), 5.15-5.03(\mathrm{~m}$, 2H), 2.32 (s, 3H); ${ }^{13} \mathrm{C}$ NMR (100 MHz, $\left.\mathrm{CDCl}_{3}\right), \delta$ (ppm) 143.31, 139.66, 137.73, $136.07,132.16,129.47,128.75,128.49,128.18,127.93,127.90,127.35,127.08$, $126.56,59.80,21.45$.

\section{Gram-scale reaction}

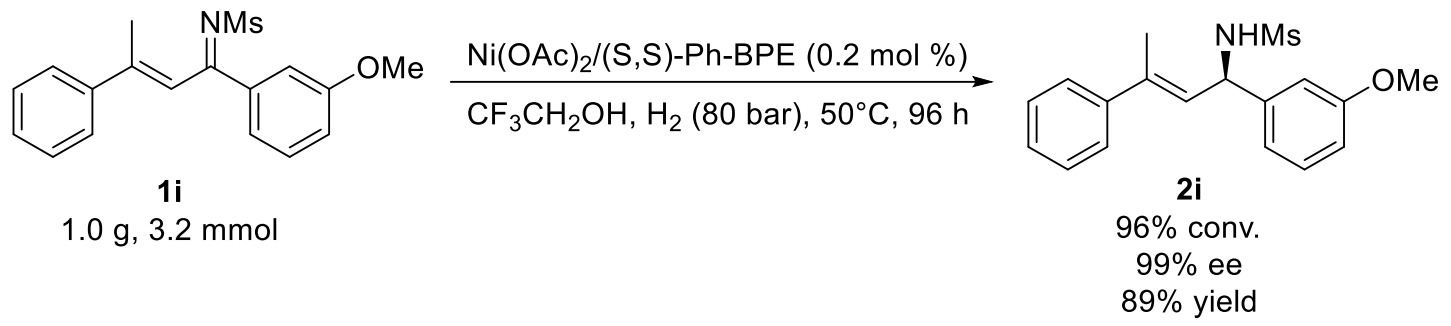

A stock solution was made by mixing $\mathrm{Ni}(\mathrm{OAc})_{2}(8.8 \mathrm{mg}, 0.05 \mathrm{mmol})$ with $(S, S)$ Ph-BPE (27.9 mg, $0.055 \mathrm{mmol})$ in a 1:1.1 molar ratio in anhydrous $\mathrm{MeOH}(1 \mathrm{~mL})$ at room temperature overnight in a nitrogen-filled glovebox. An aliquot of the catalyst solution (128 uL, $0.0064 \mathrm{mmol}$ ) was transferred by syringe into the vial charged with 1i $(1.0 \mathrm{~g}, 3.2 \mathrm{mmol})$ in anhydrous $\mathrm{CF}_{3} \mathrm{CH}_{2} \mathrm{OH}(5.0 \mathrm{~mL})$. The vial was subsequently transferred into an autoclave into which hydrogen gas was charged. The reaction was then stirred under $\mathrm{H}_{2}(80 \mathrm{~atm})$ at $50{ }^{\circ} \mathrm{C}$ for $96 \mathrm{~h}$. The hydrogen gas was released slowly and carefully. The solution was concentrated and then the residue was purified by flash column chromatography on silica gel to afford $0.95 \mathrm{~g} \mathbf{2 i}$ in $89 \%$ yield and $99 \%$ ee. 


\section{Reference}

[1] Hao, W.; Harenberg, J.; Wu, X.; MacMillan, S.; Lin, S. J. Am. Chem. Soc. 2018, $140,3514-3517$.

[2] Lempenauer, L.; DuÇach, E.; LemiHre, G. Chem. Eur. J. 2017, 23, 10285-10288.

[3] Hu, Z.; Liu, J.; Li, G.; Dong, Z.; Li, W. J. Chin. Chem. Soc. 2004, 51, 581-583.

[4] Zhang, T.; Jiang, J.; Yao, L.; Geng, H.; Zhang, X. Chem. Commun. 2017, 53, 92589261.

[5] Lu, S.; Bolm, C. Chem. Eur. J. 2008, 14, 7513-7516.

[6] Nishikawa, Y.; Yamamoto, H. J. Am. Chem. Soc. 2011, 133, 8432-8435.

[7] Ott, A.; Goshey, C.; Topczewski, J. J. Am. Chem. Soc. 2017, 139, 7737-7740.

[8] Chen, X.; Zhou, H.; Zhang, K.; Li, J.; Huang, H. Org. Lett. 2014, 16, 3912-3915.

[9] Jiang, K.; Tiwari, B.; and Chi, Y. Org. Lett. 2012, 14, 2382-2385.

[10] Espinosa, M.; Blay, G.; Cardona, L.; and Pedro, J. Chem. Eur. J. 2013, 19, 14861 $-14866$.

[11] Vantomme, G.; Jiang, S.; and Lehn, J. J. Am. Chem. Soc. 2014, 136, 9509-9518.

[12] He, Z.; Han, B.; Li, R.; Wub, L.; Chen, Y. Org. Biomol. Chem., 2010, 8, 755-757.

[13] Simal, C.; Lebl, T.; Slawin, A.; Smith, A. Angew. Chem. Int. Ed. 2012, 51, $3653-$ 3657.

[14] Sandrinelli, F.; Perrio, S.; Beslin, P. J. Org. Chem. 1997, 62, 8626-8627.

[15] Esquivias, J.; Arrayas, R.; Carretero, J. J. Org. Chem. 2005, 70, 7451-7454.

[16] Huang, Y.; Chew, R.; Pullarkat, S.; Li, Y.; Leung, P. J. Org. Chem. 2012, 77, 6849-6854.

[17] Carmen S., Tomas L., Alexandra M. Z. S., Andrew D. S, Angew. Chem. Int. Ed. 2012, 51, $3653-3657$.

[18] Bower, J. F.; Jumnah, R.; Williams, A. C.; Williams, J. M. J. J. Chem. Soc., Perkin Trans. 1, 1997, 1411-1420 
VI. $\quad{ }^{1} \mathrm{H}$ NMR and ${ }^{13} \mathrm{C}$ NMR spectra

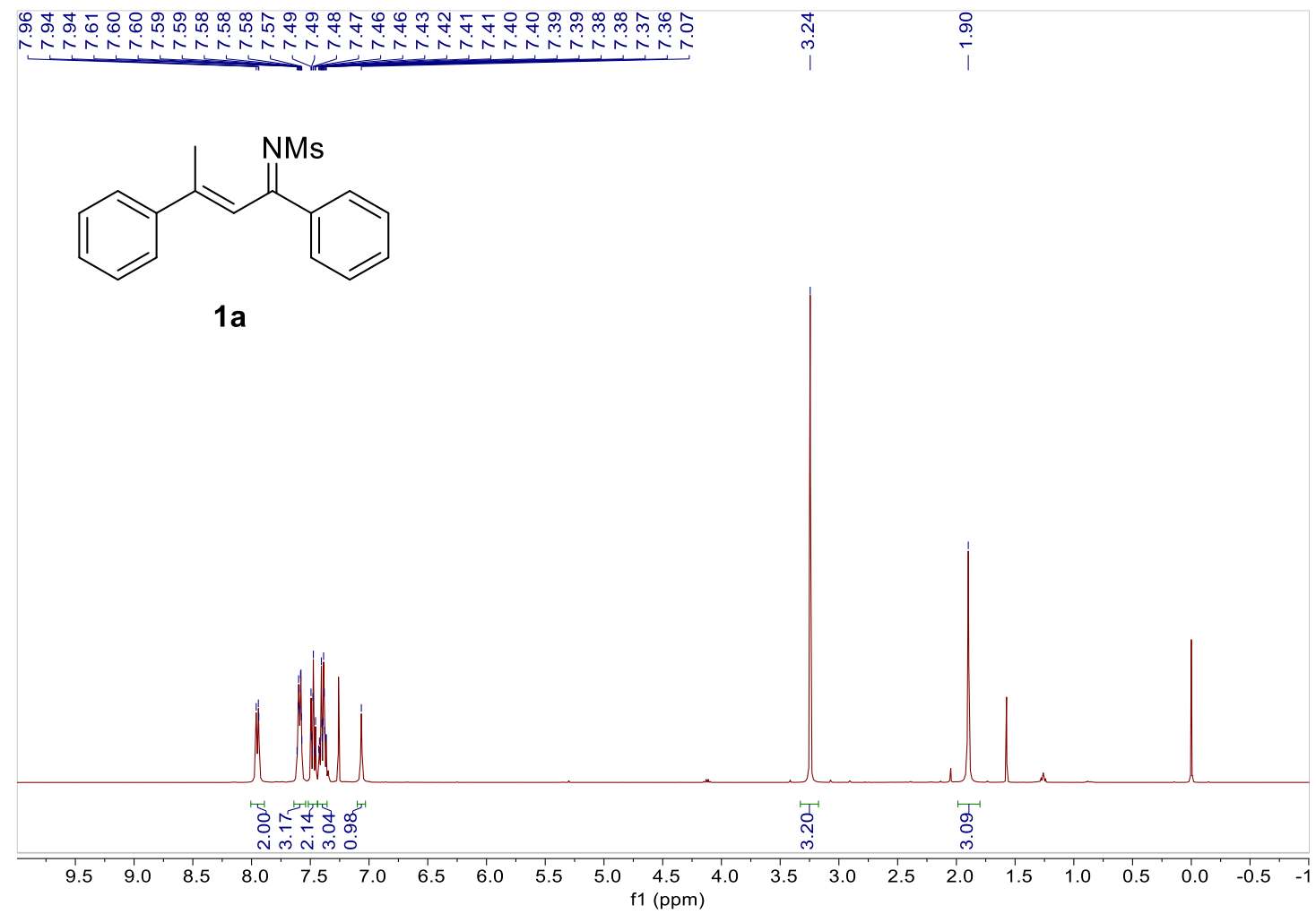



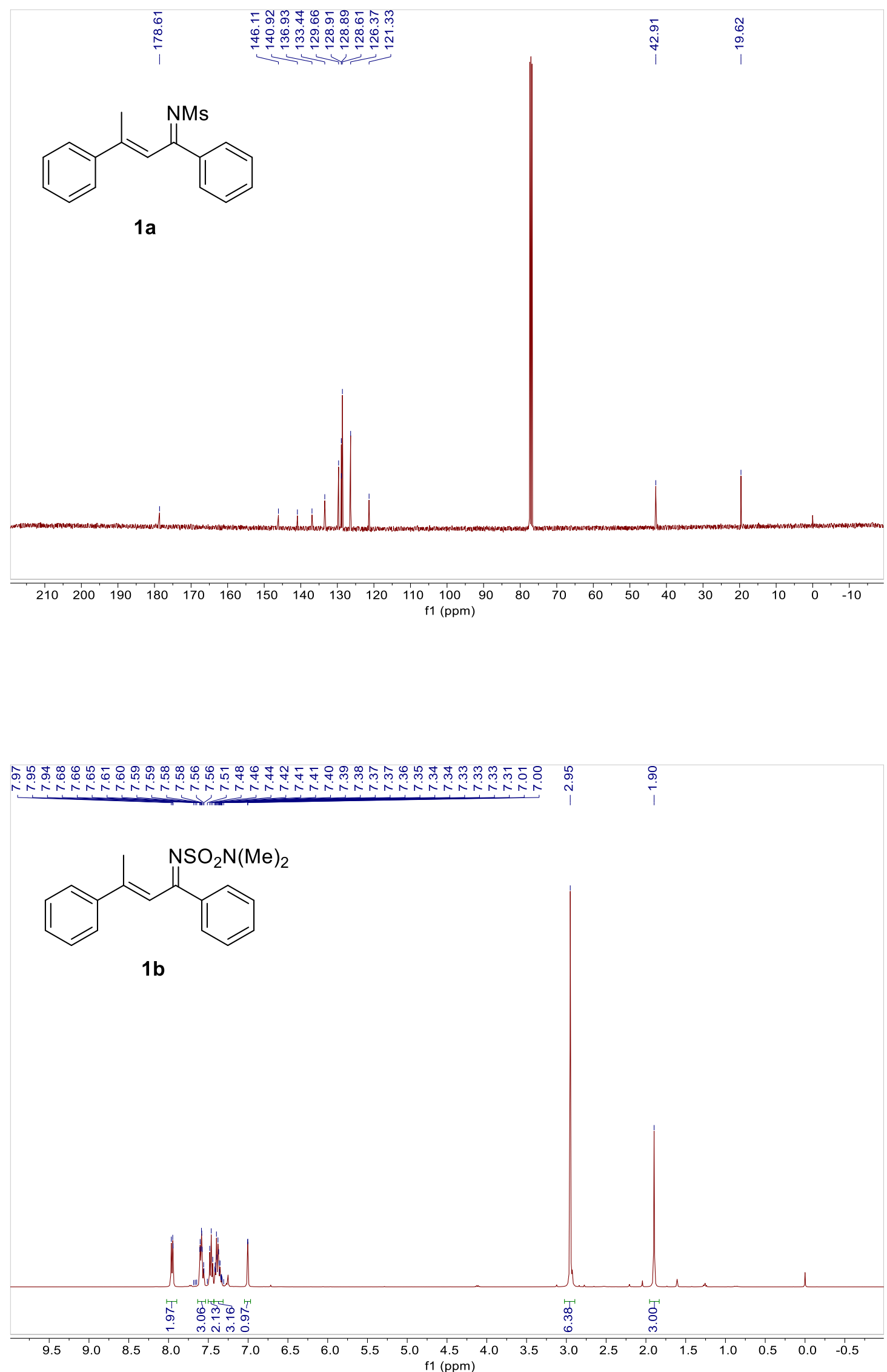

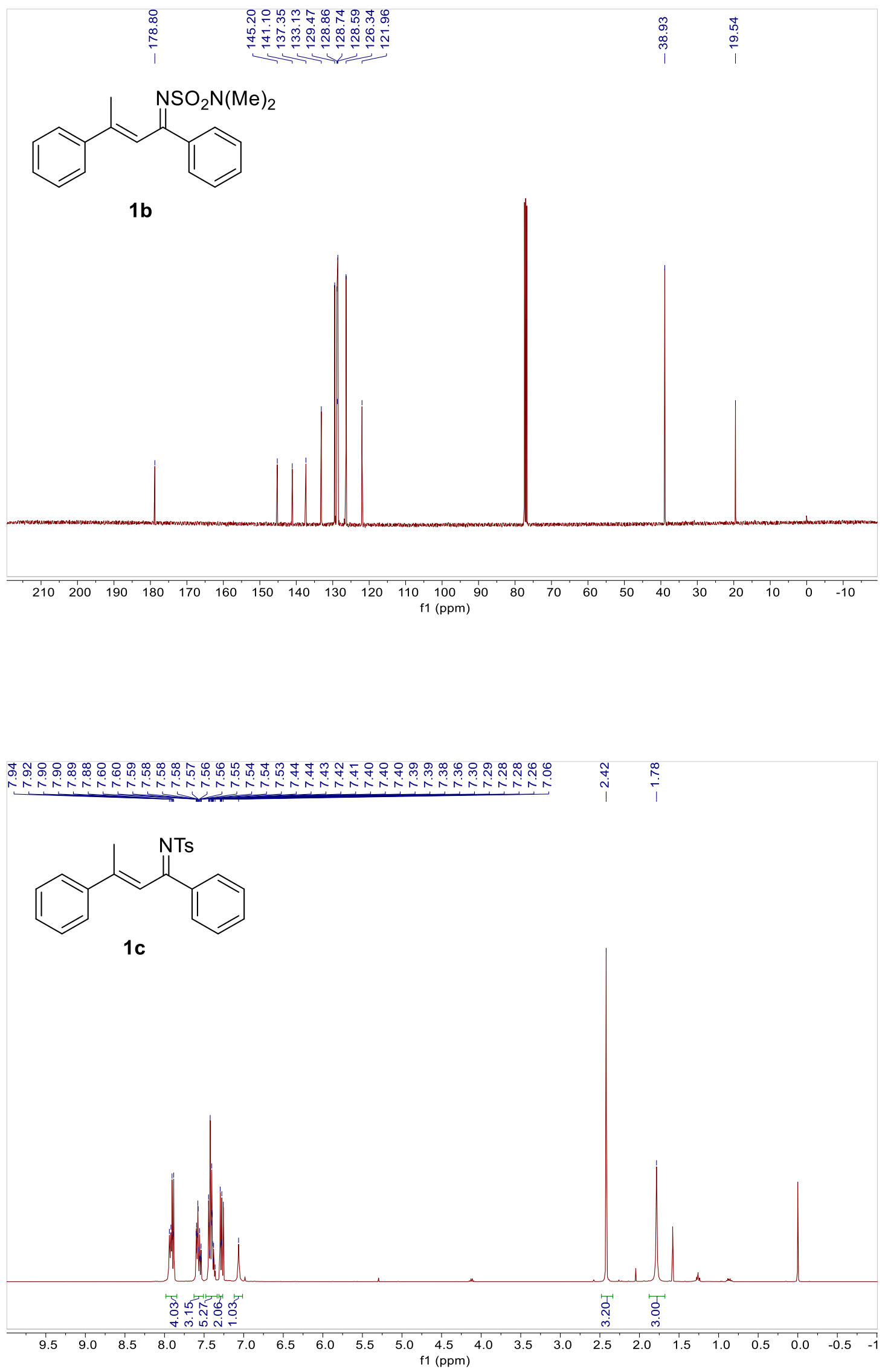

25 

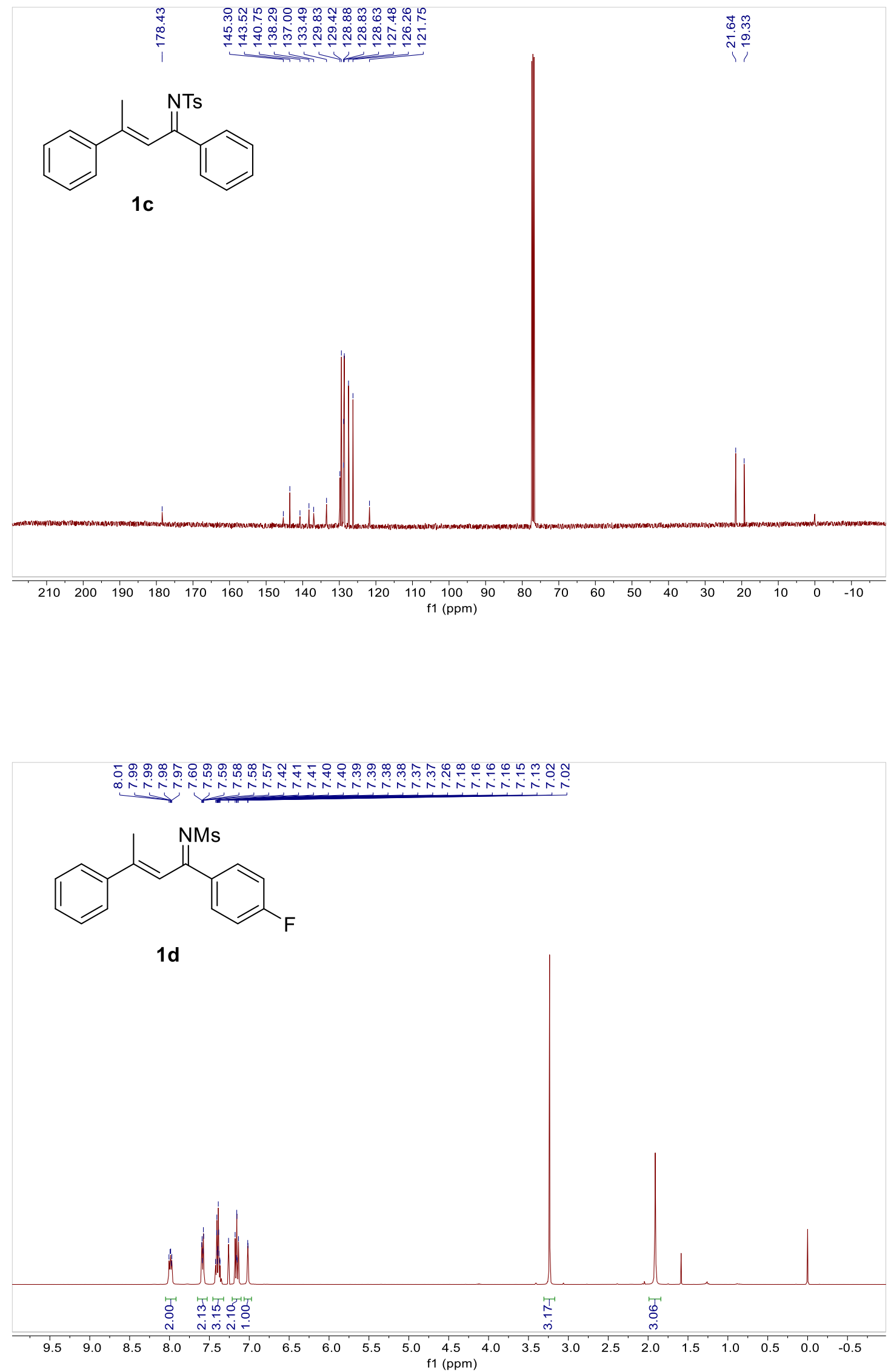

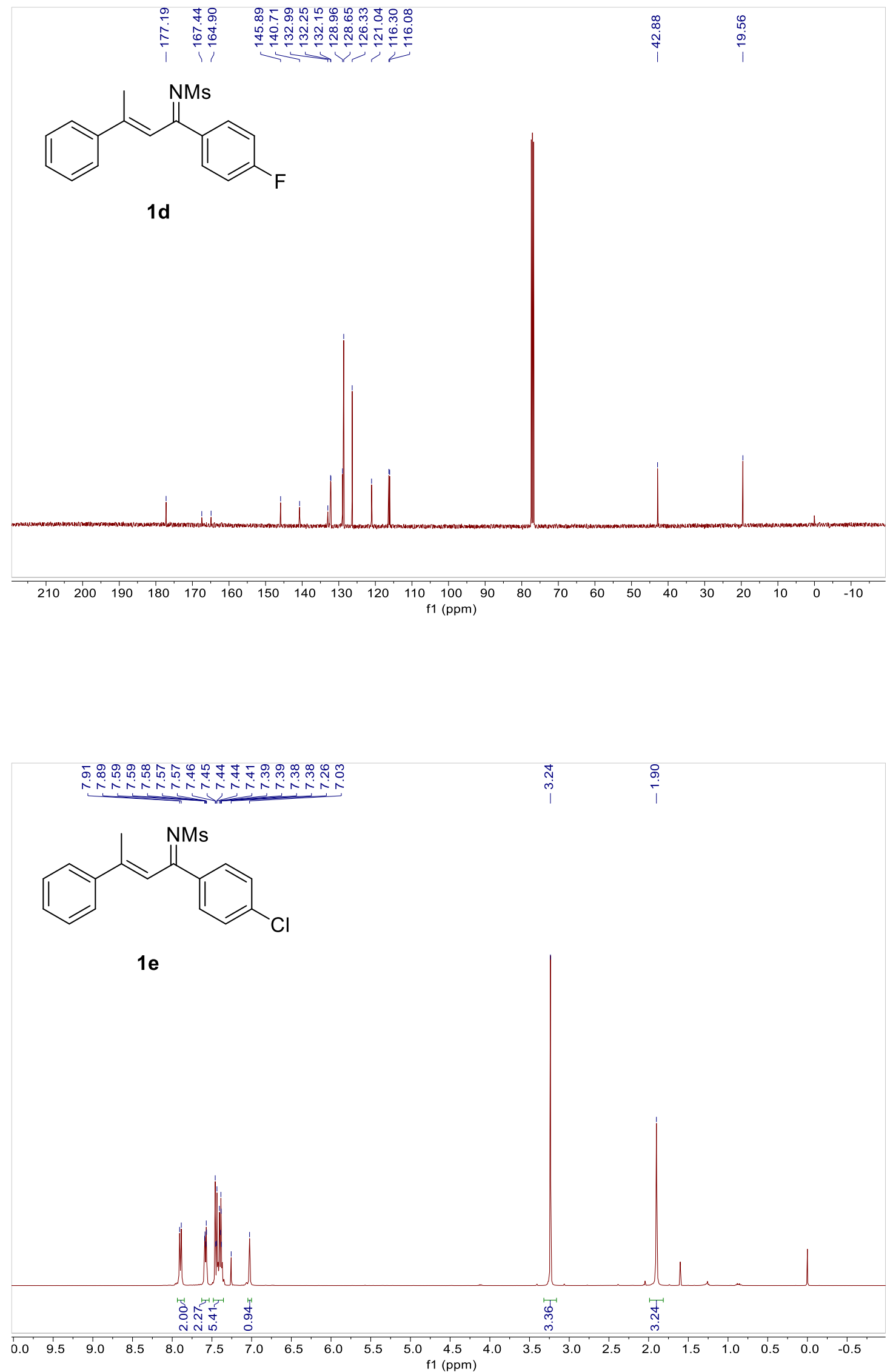

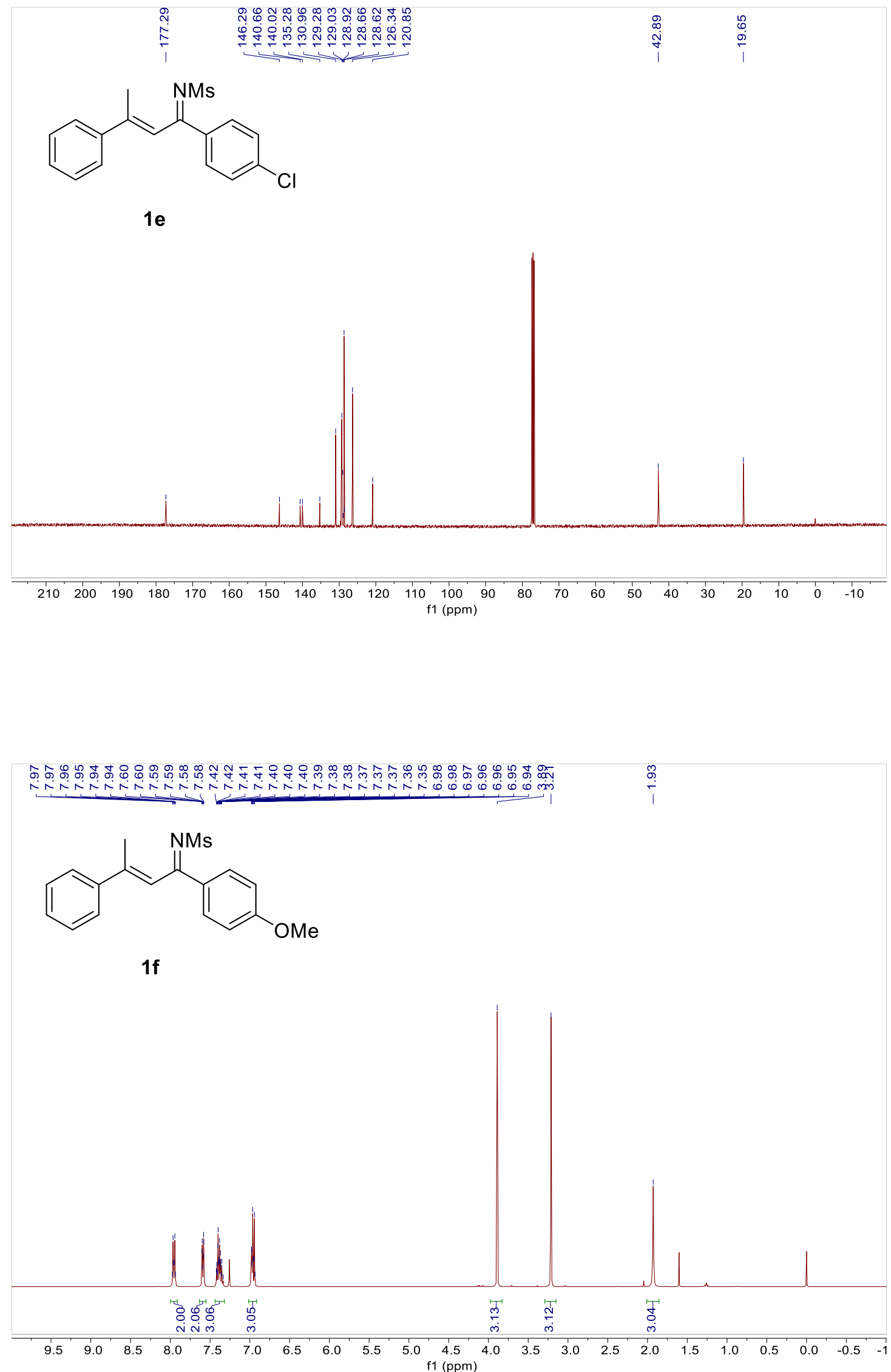

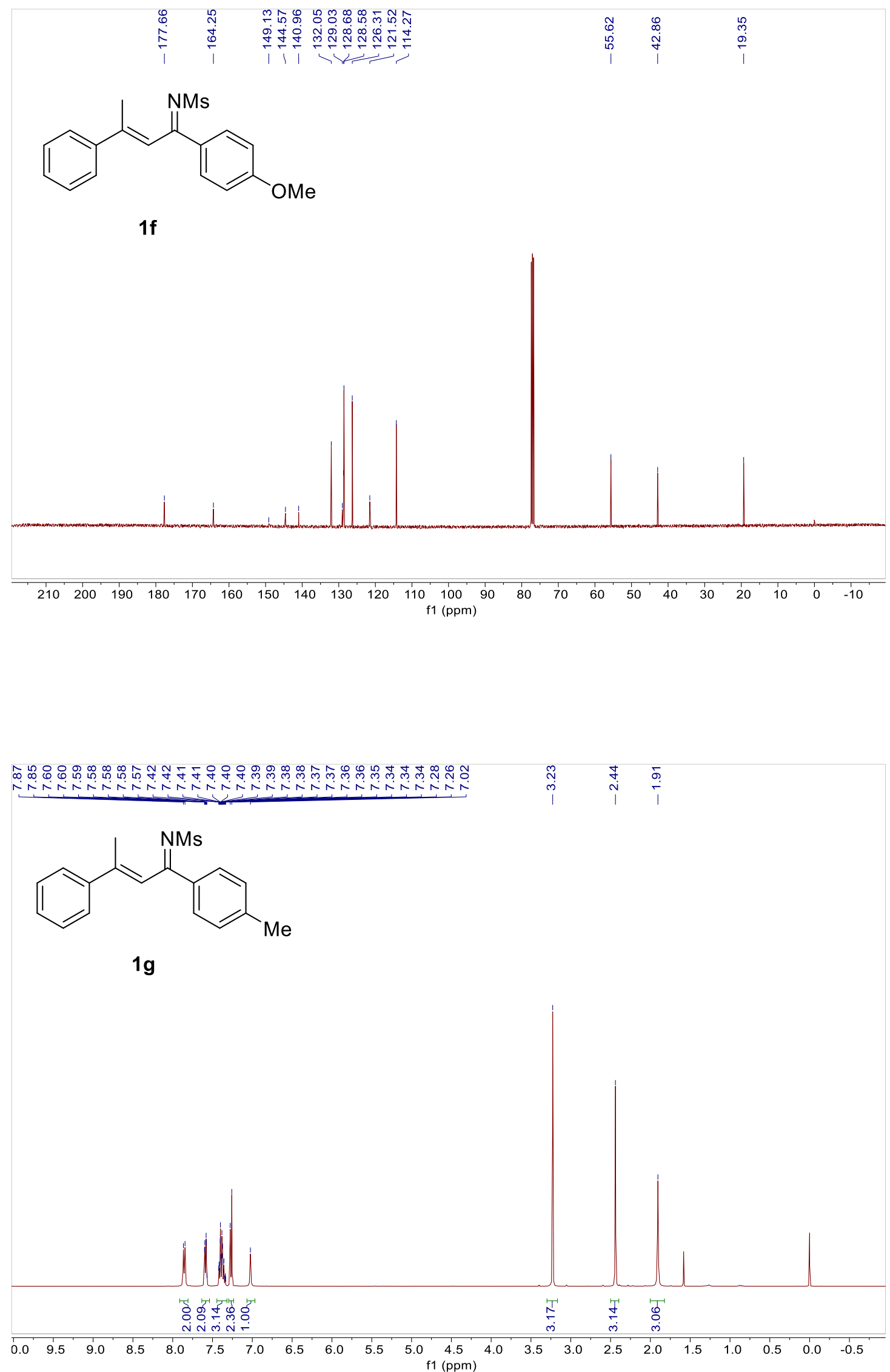


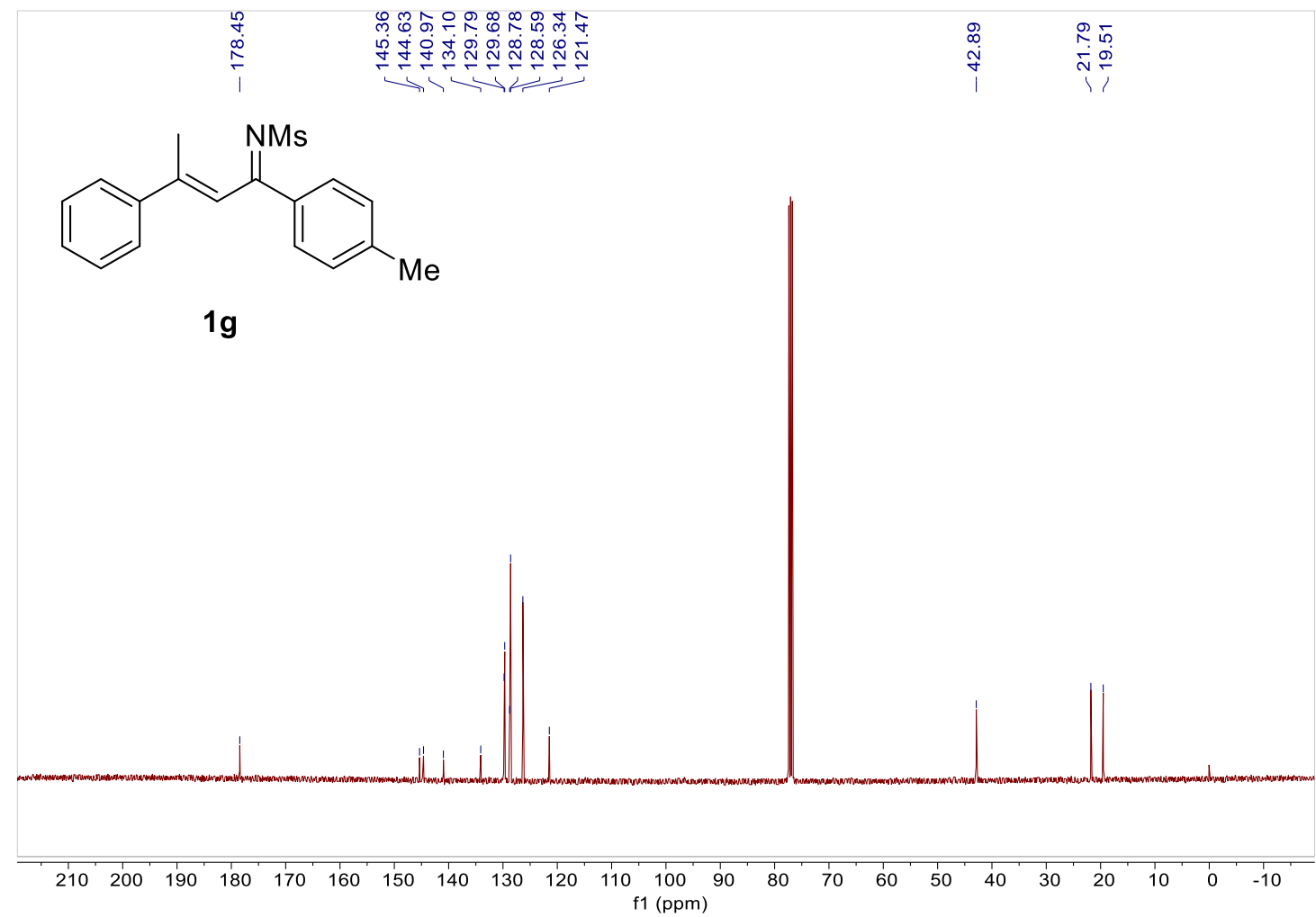

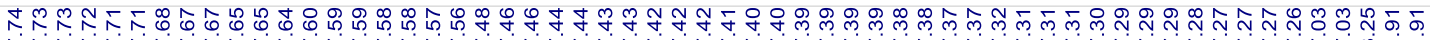

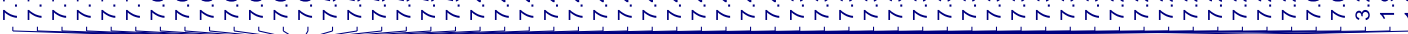<smiles>C/C(=C\C(=N)c1cccc(F)c1)c1ccccc1</smiles>

$1 \mathrm{~h}$

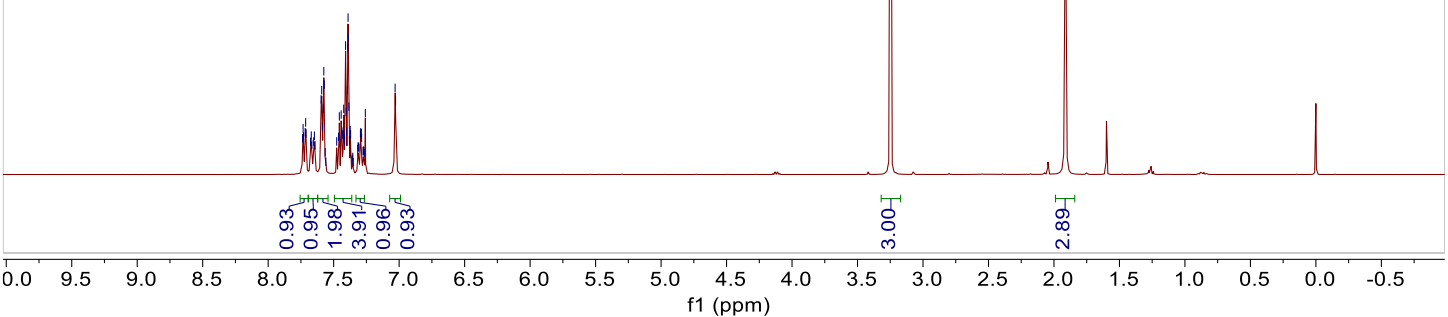




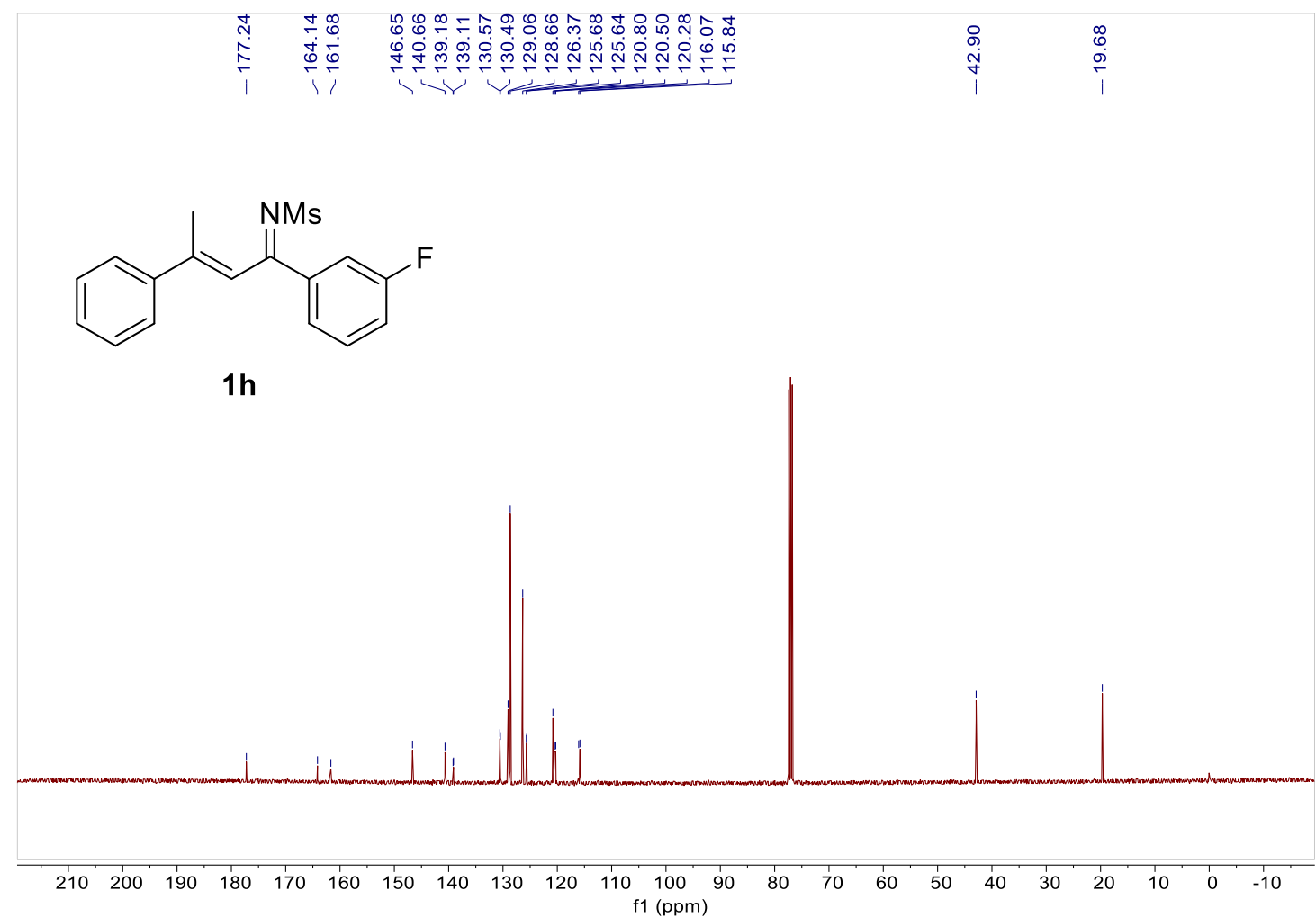

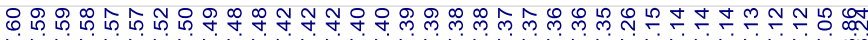

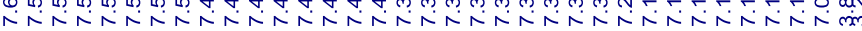<smiles>C/N=C(\C=C(C)c1ccccc1)c1cccc(OC)c1</smiles>

$1 \mathrm{i}$

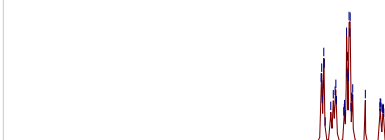

我

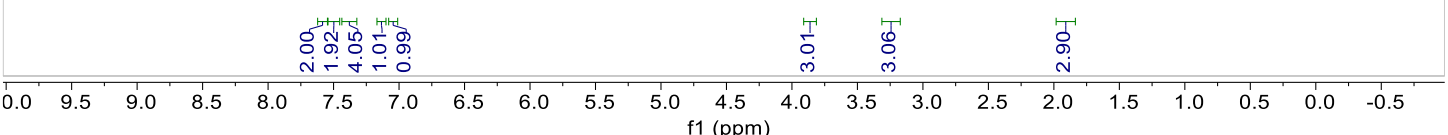




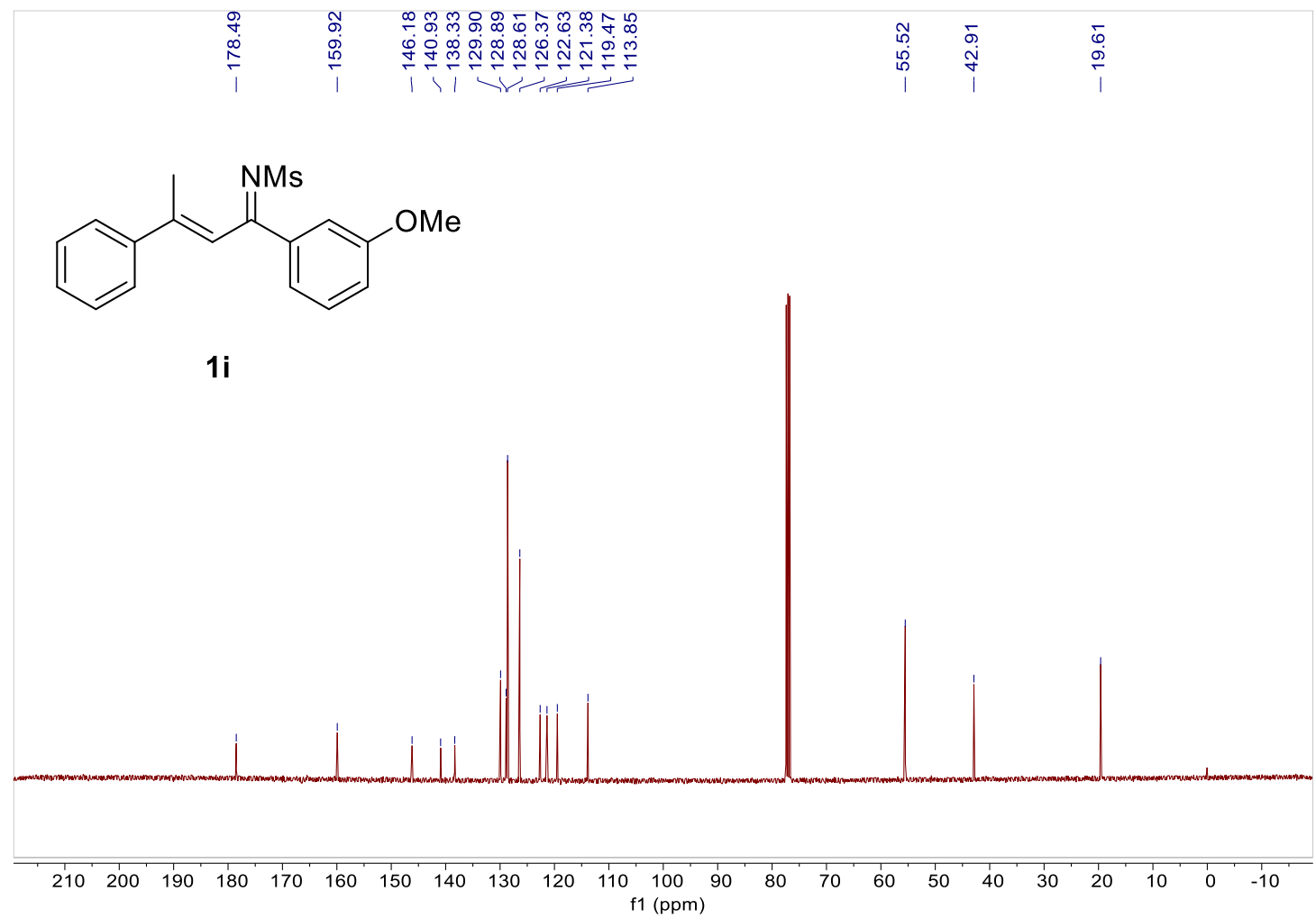

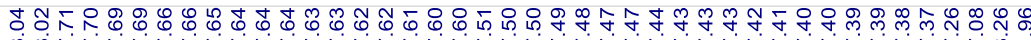

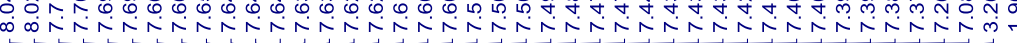<smiles>CN=C(/C=C(\C)c1ccccc1)c1ccccc1-c1ccccc1</smiles>

$1 \mathrm{j}$

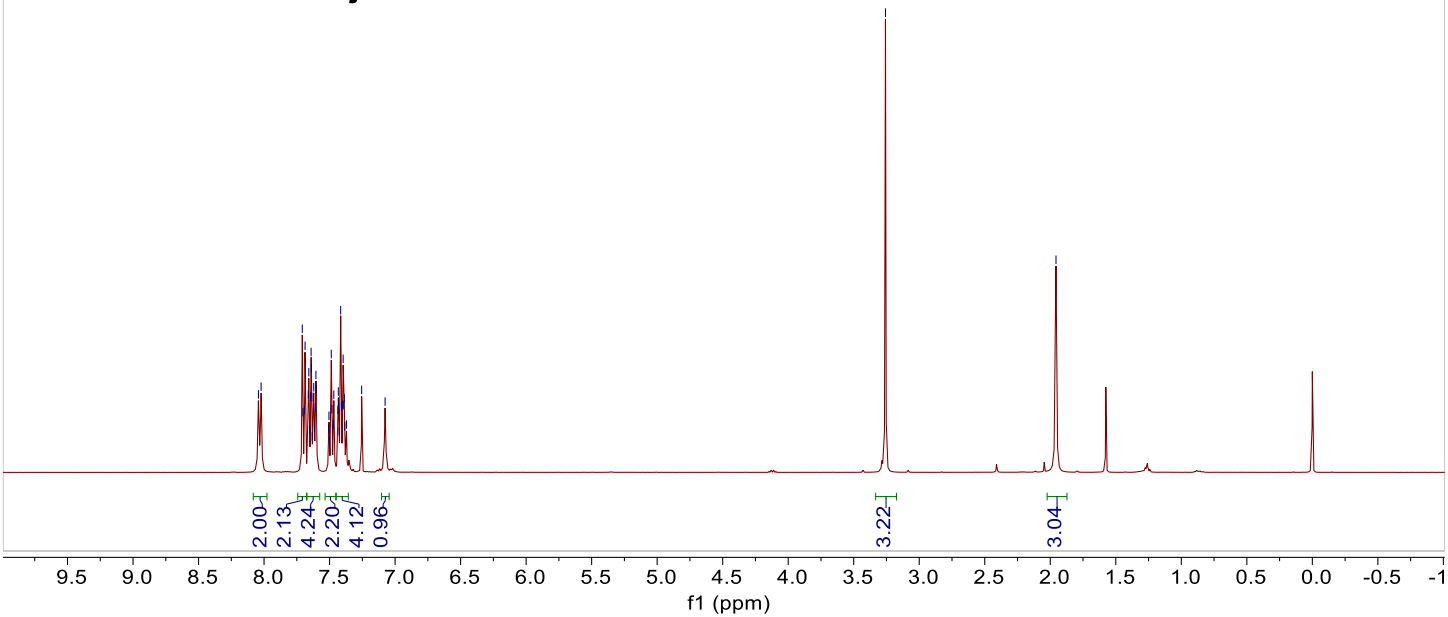



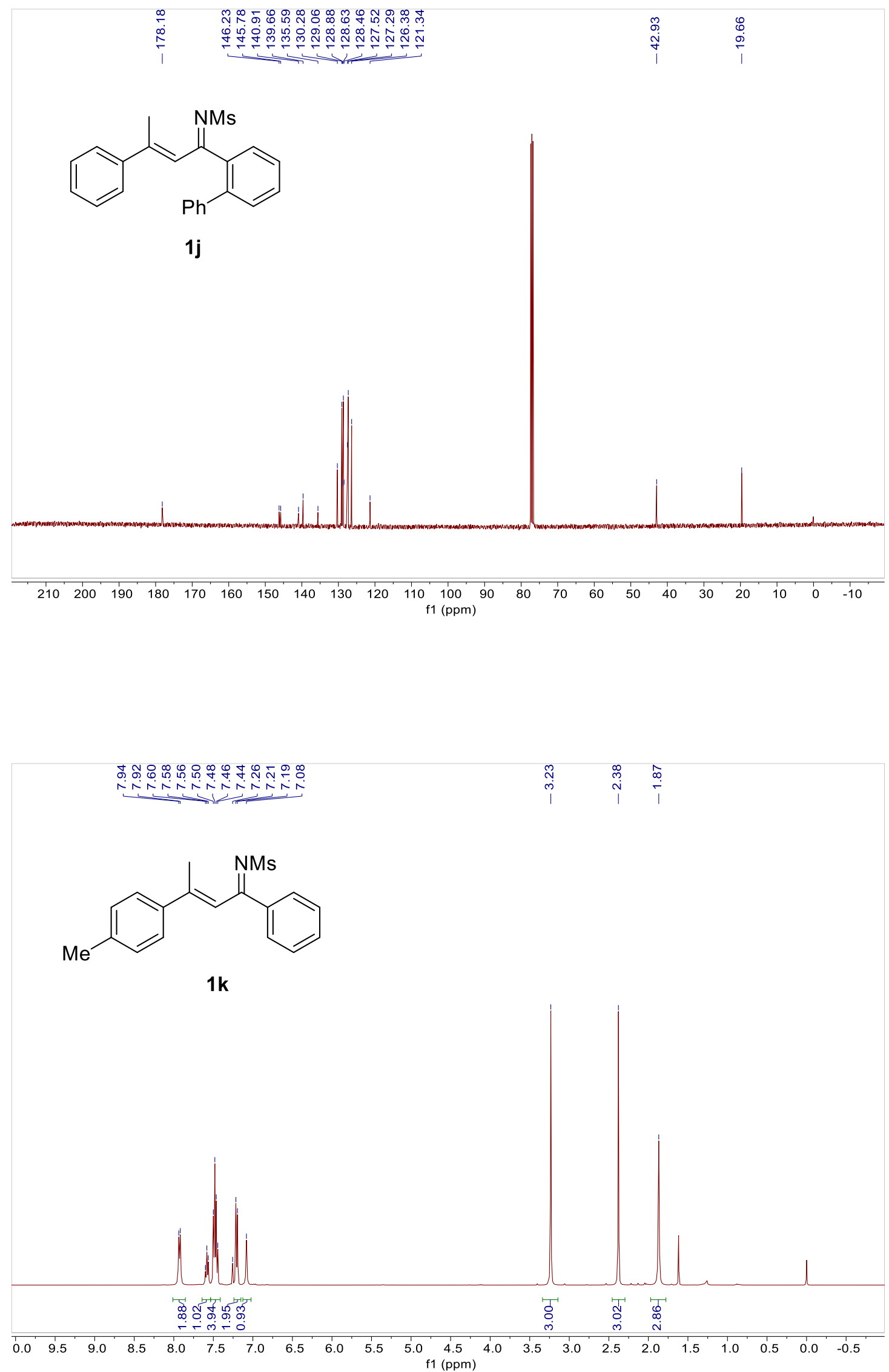

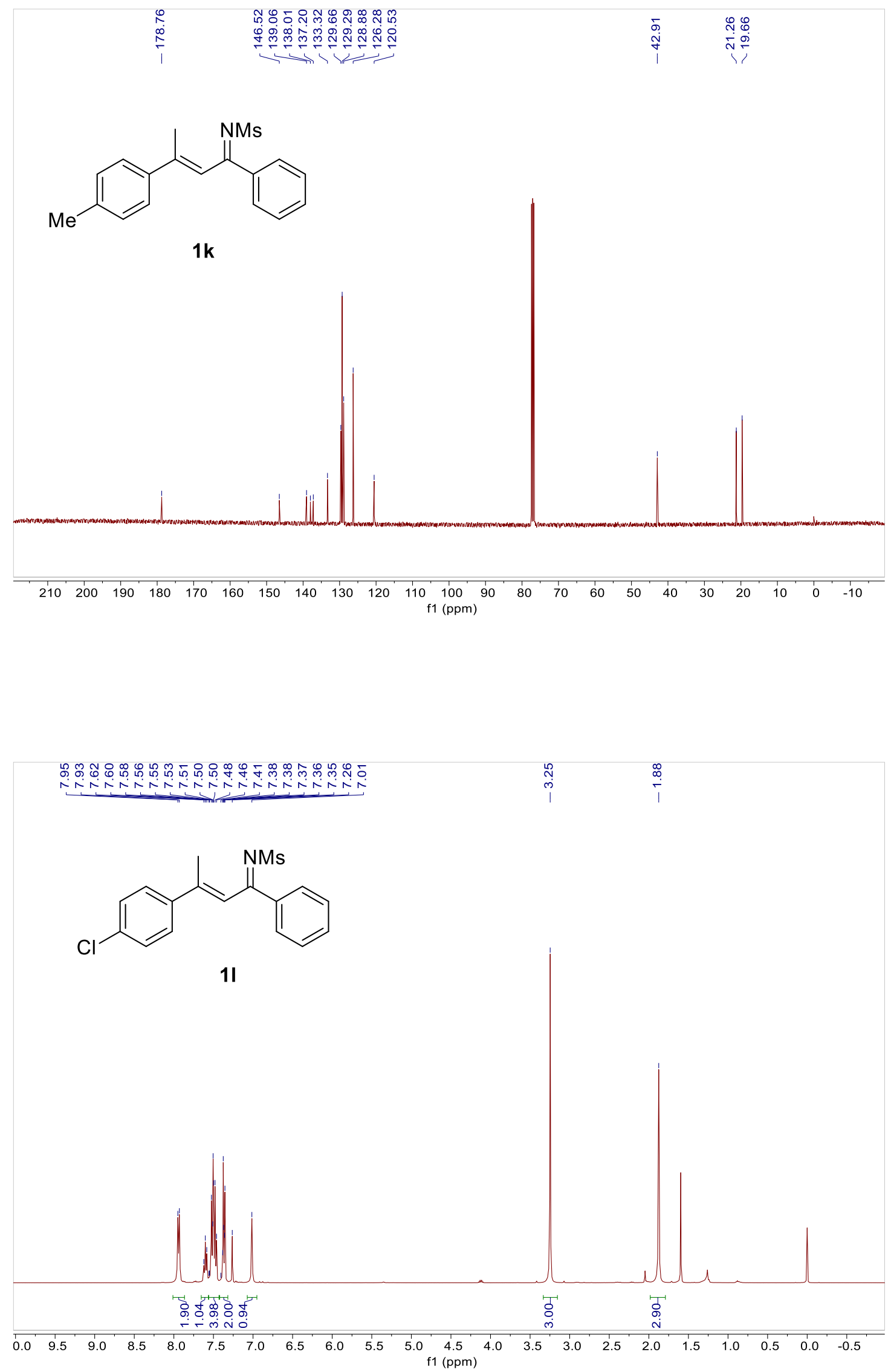

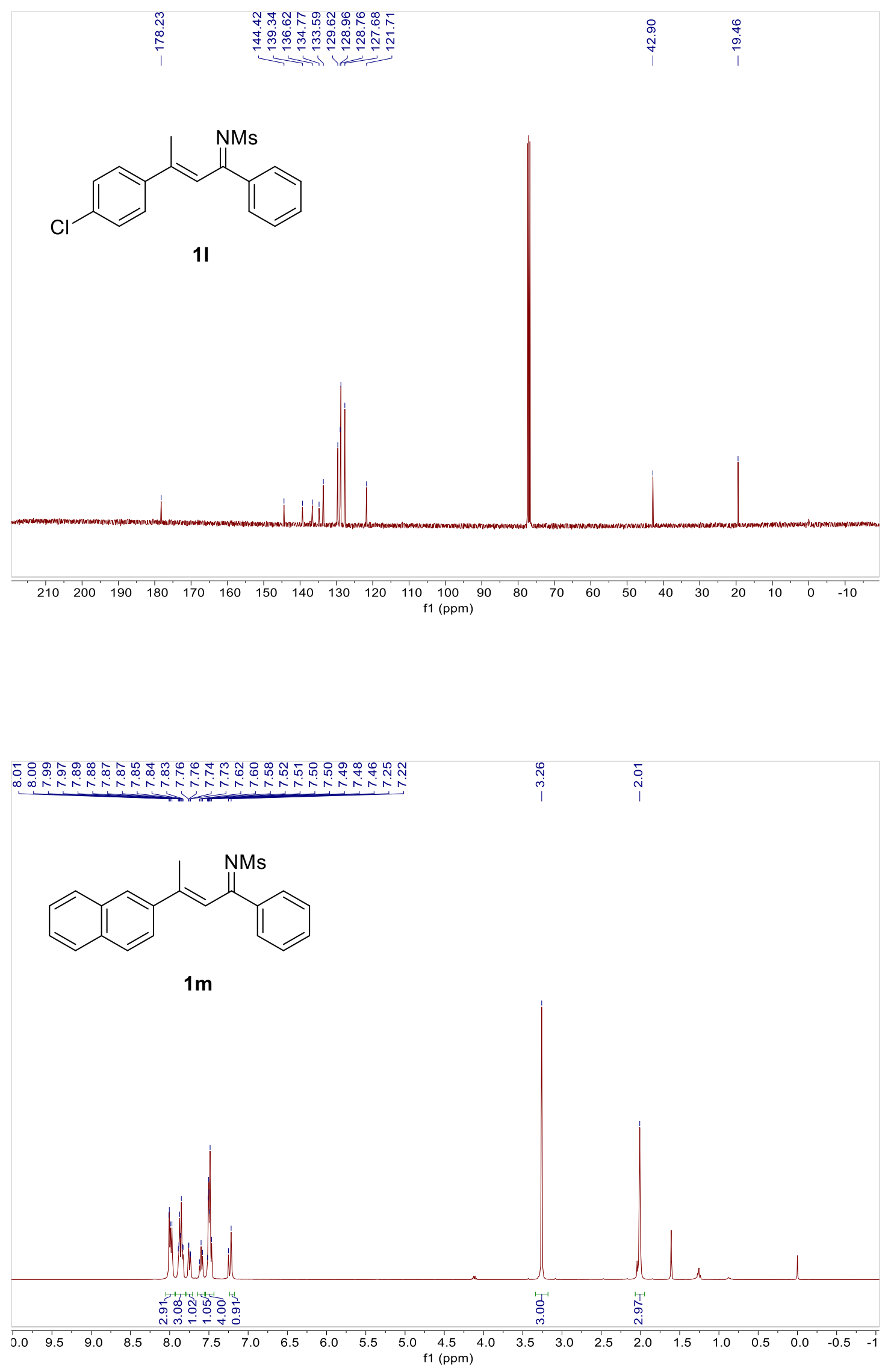

35 

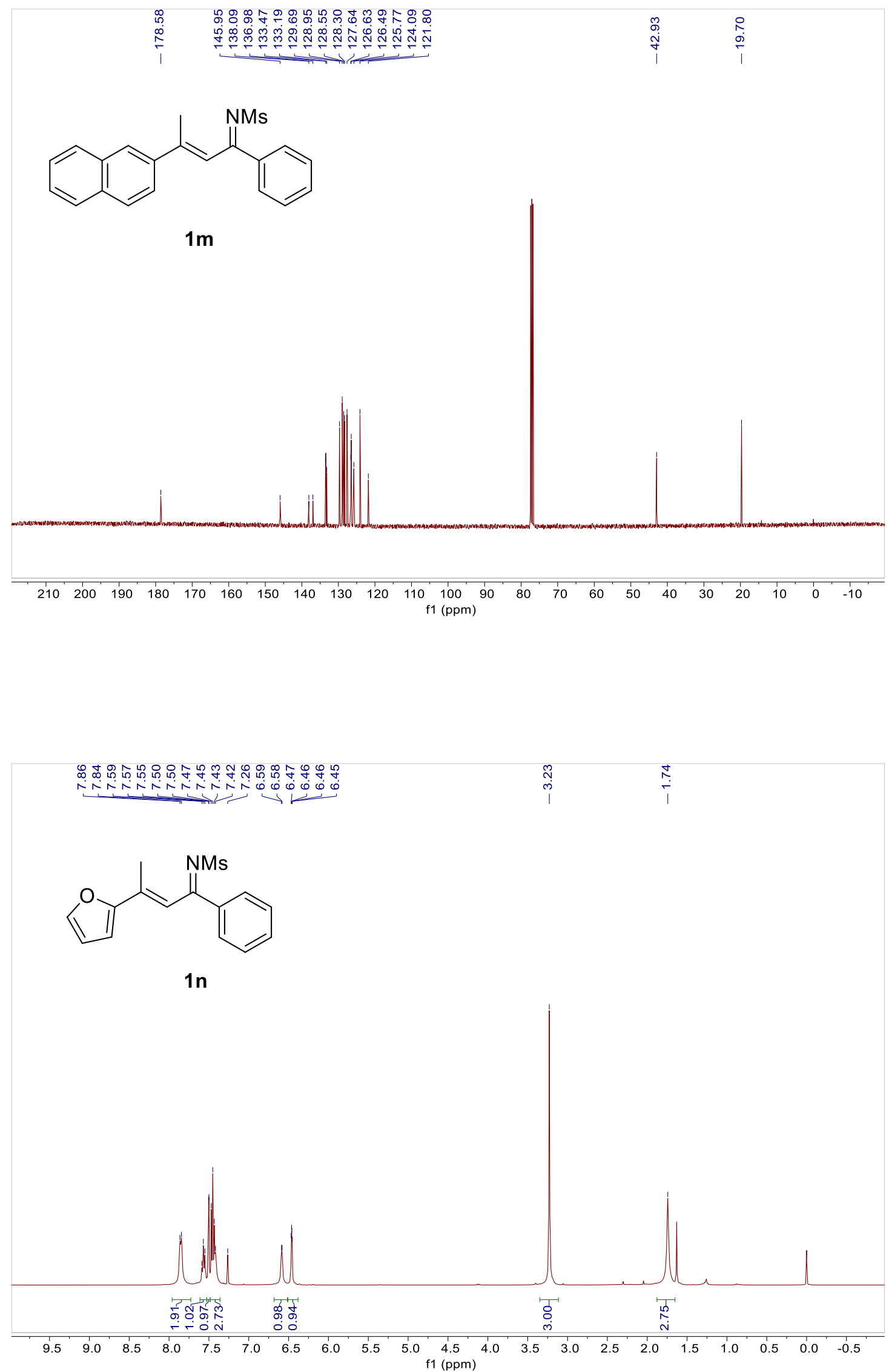

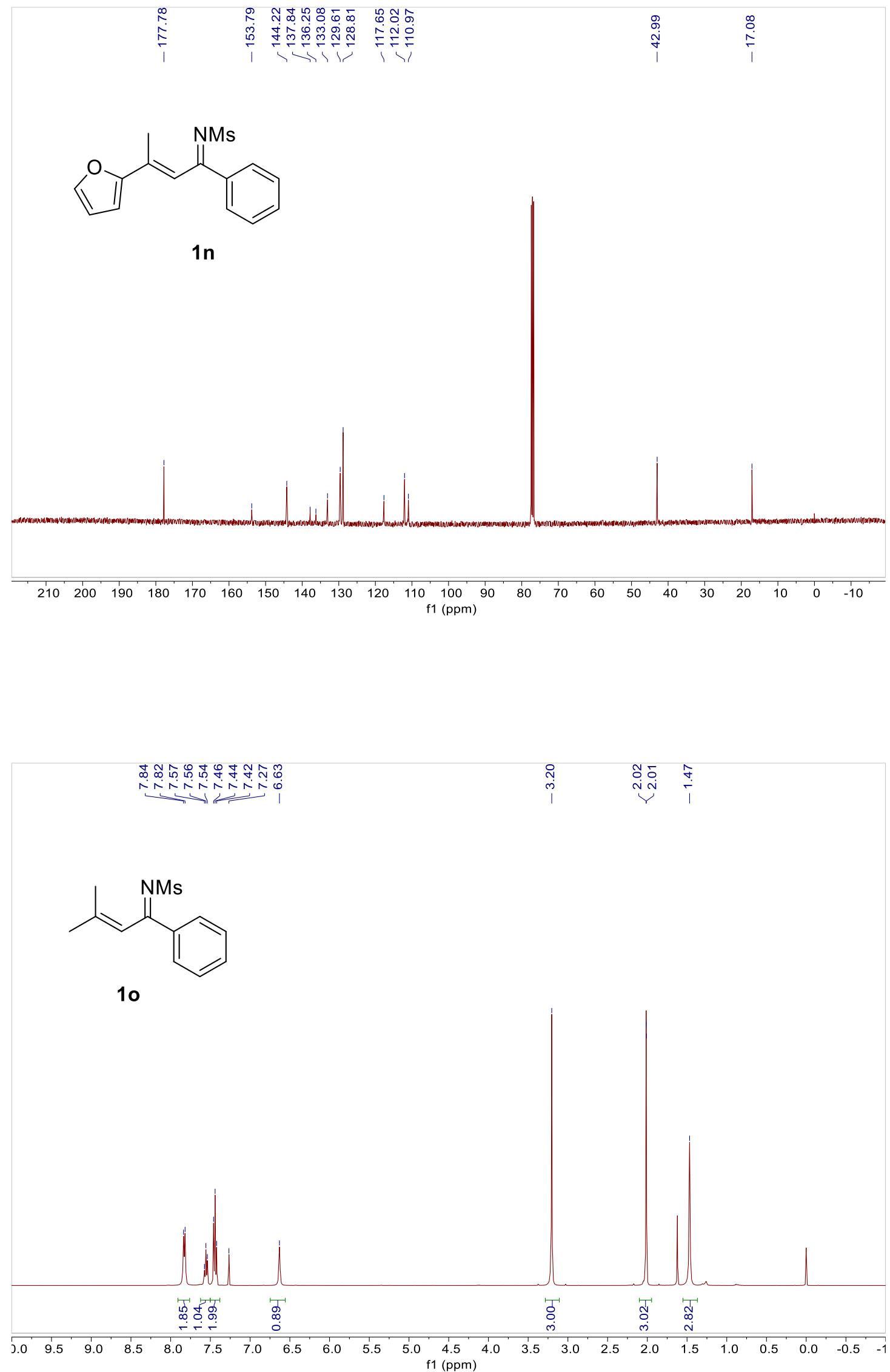


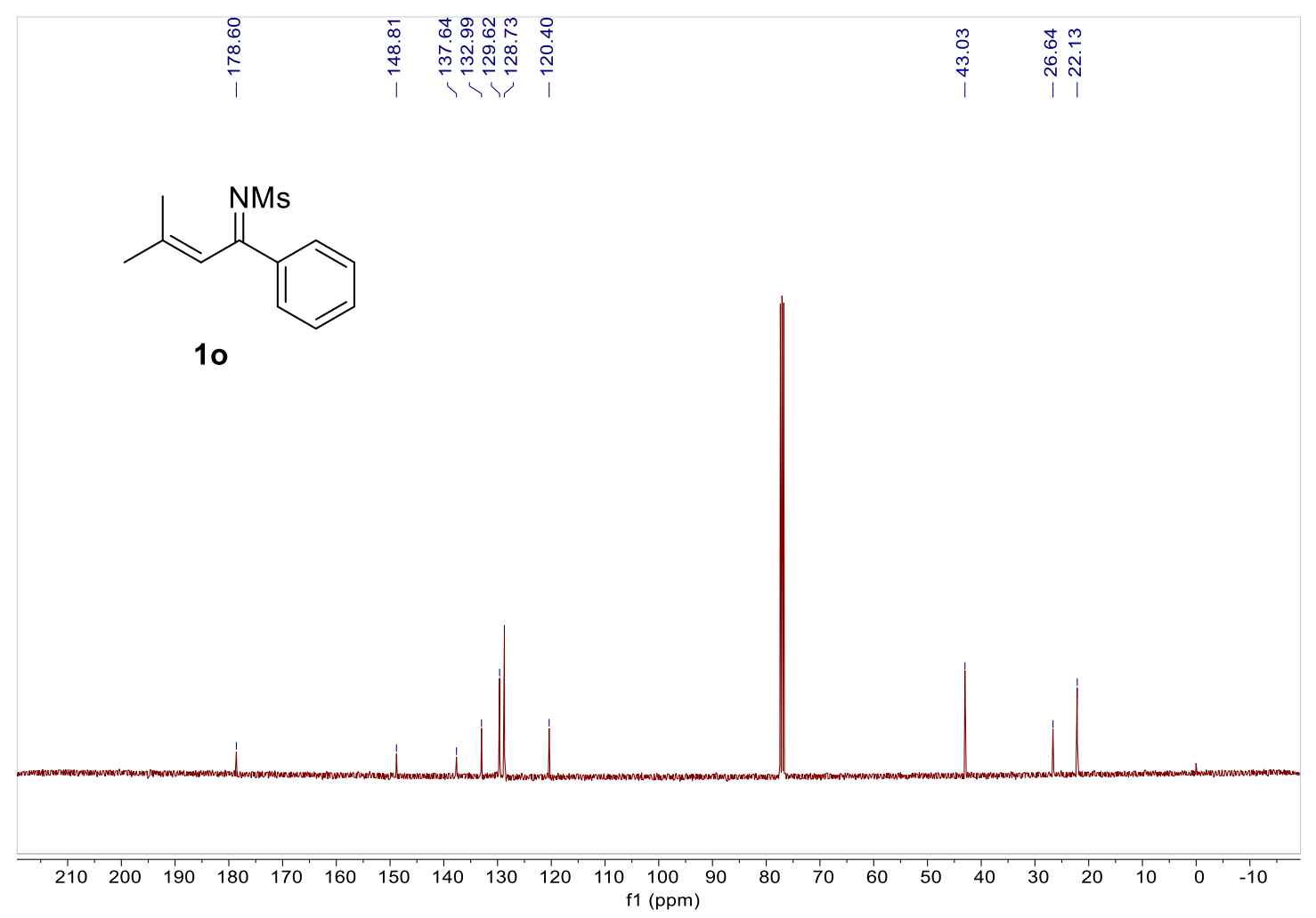

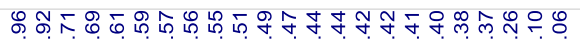

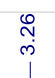
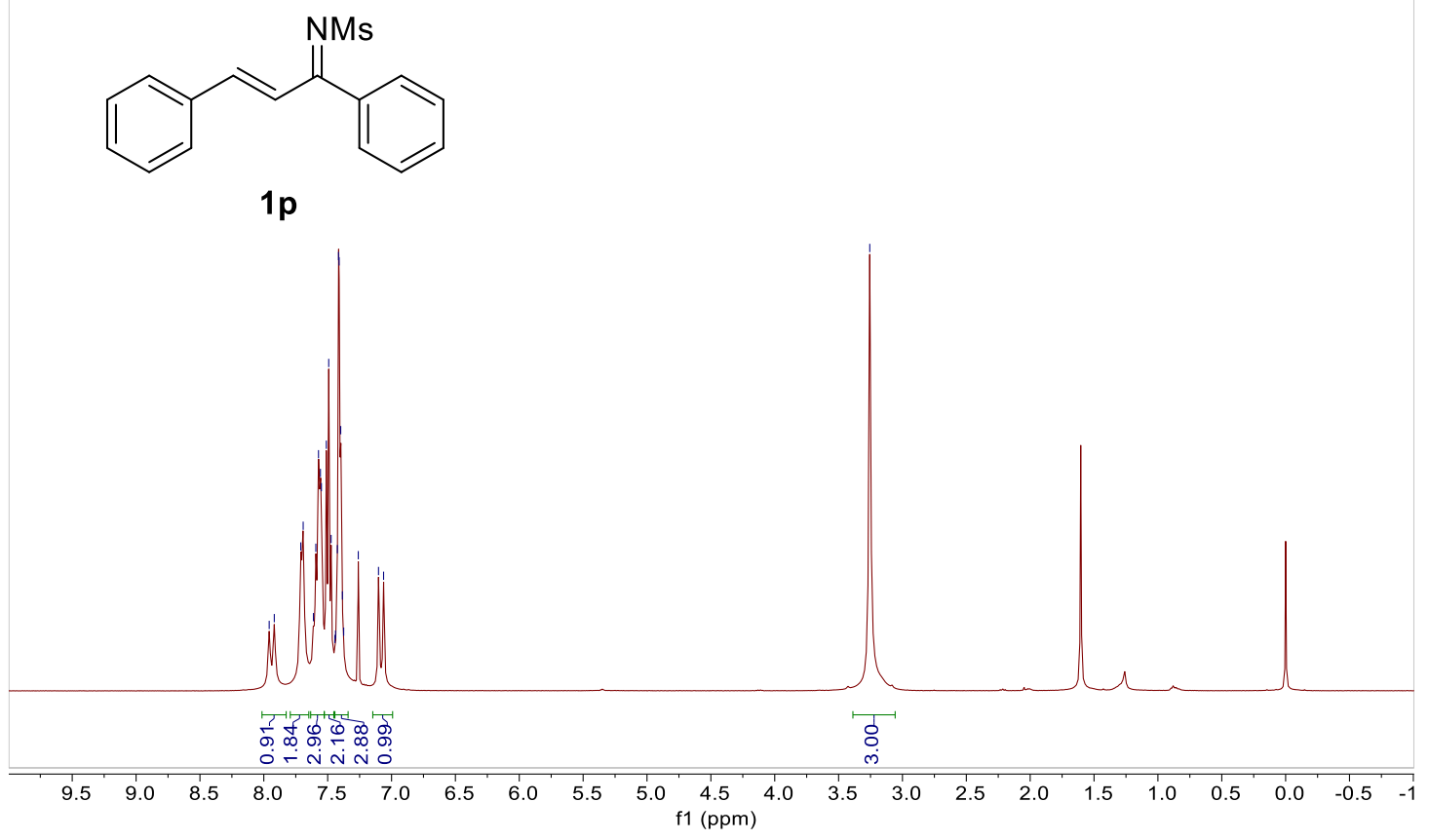

38 

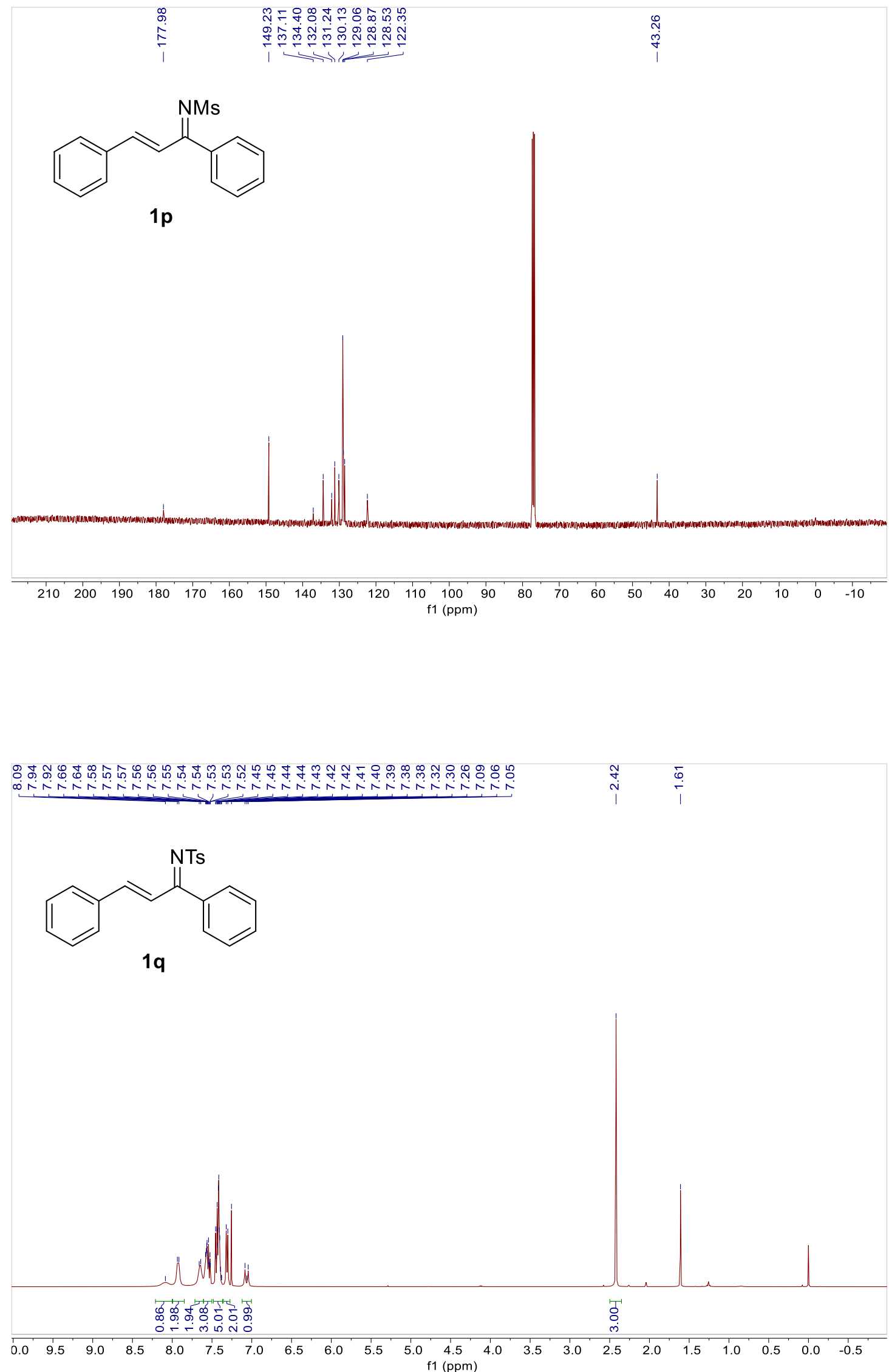


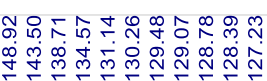

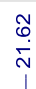<smiles>[N+]=C(/C=C/c1ccccc1)c1ccccc1</smiles>

$1 q$

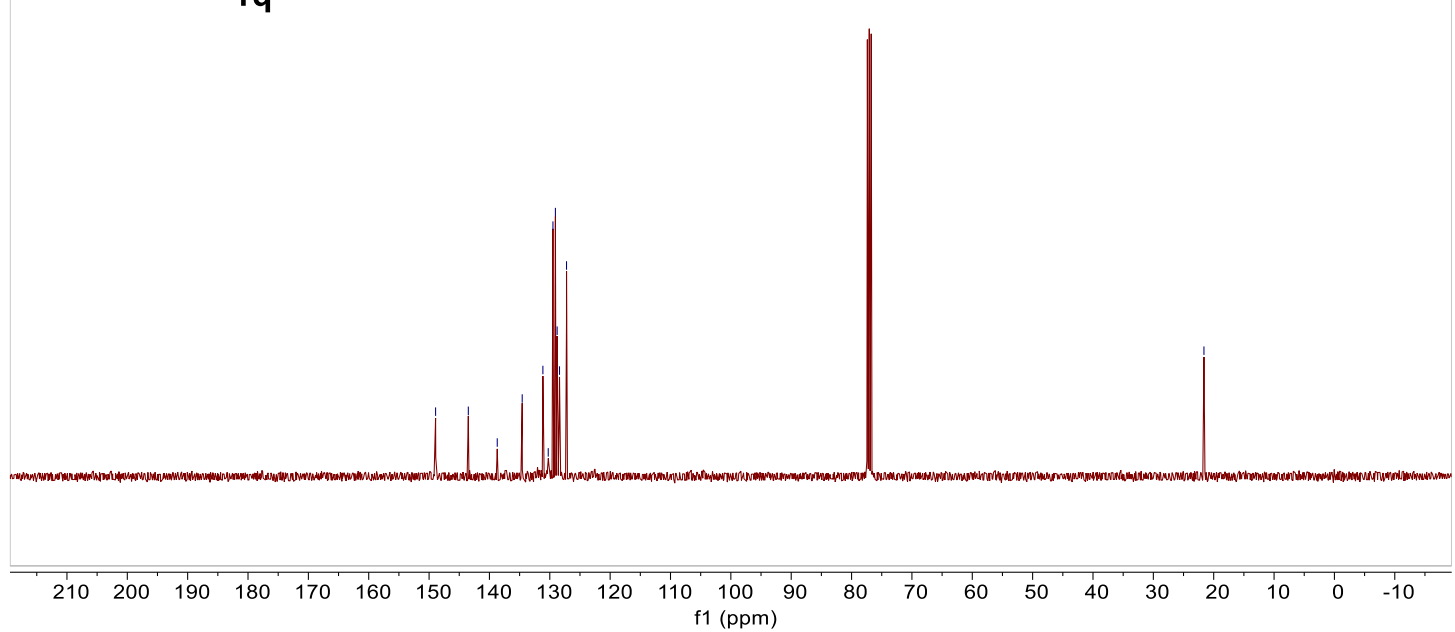

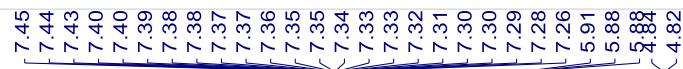

สูก บู<smiles>C/C(=C\[C@H](c1ccccc1)N(C)C)c1ccccc1</smiles>

2a

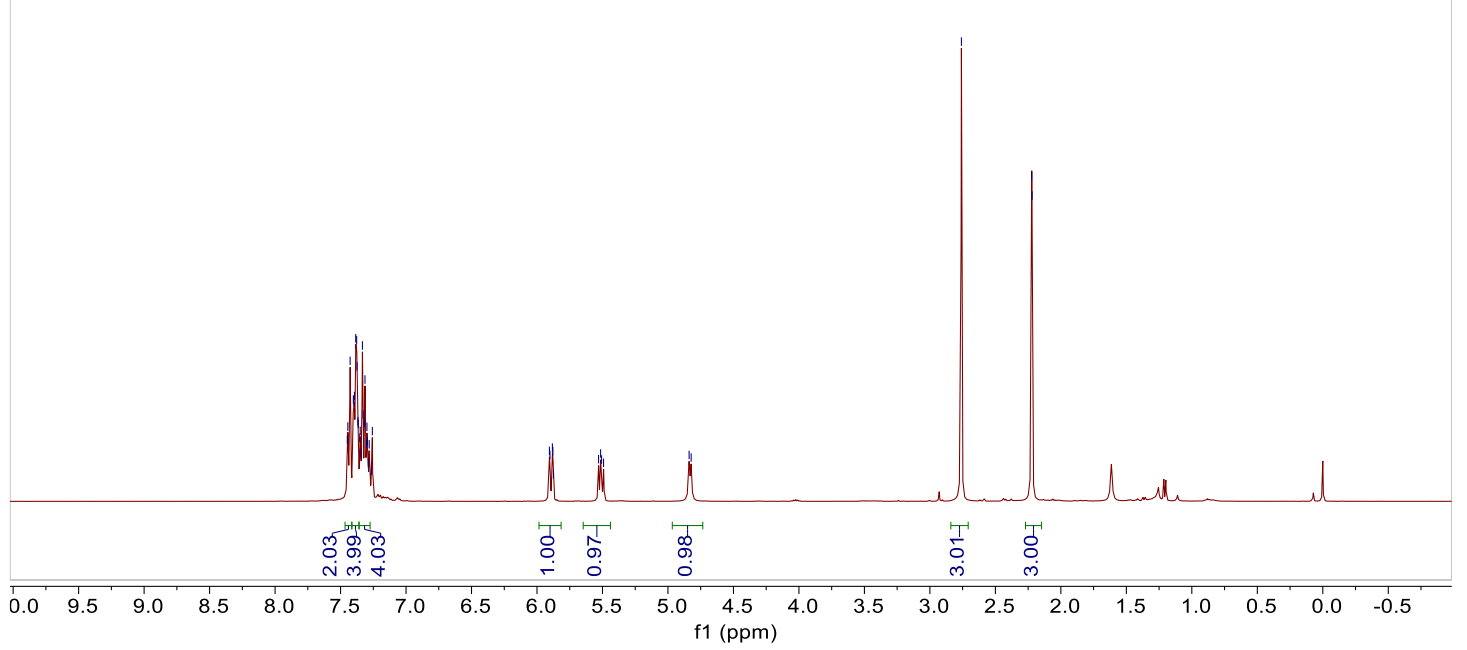

40 


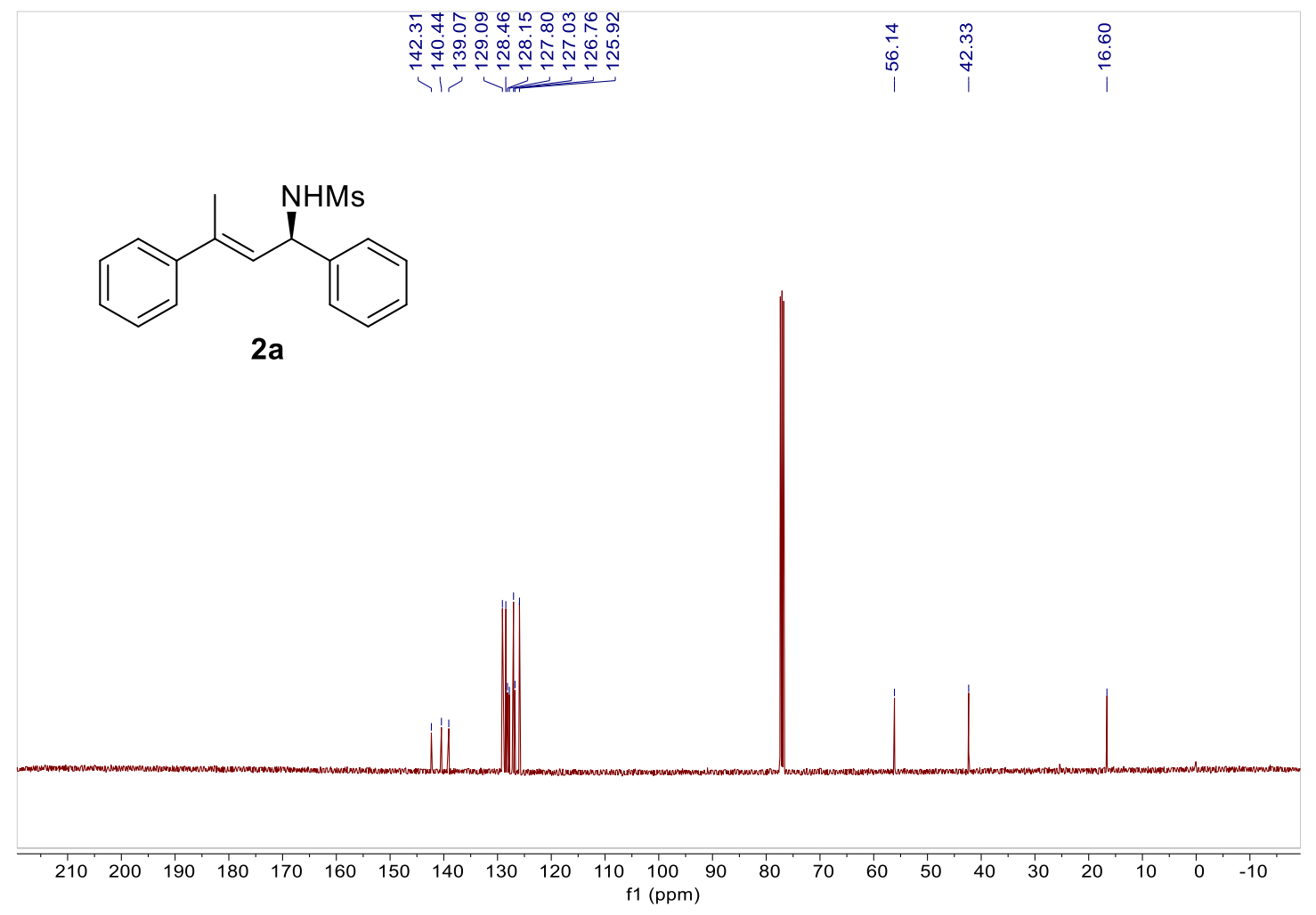

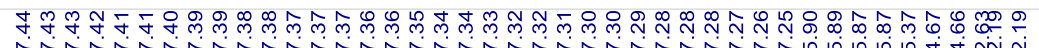

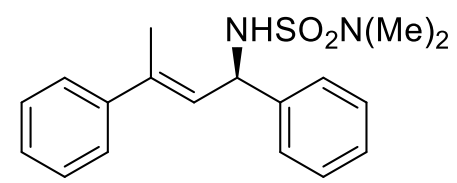

$2 b$

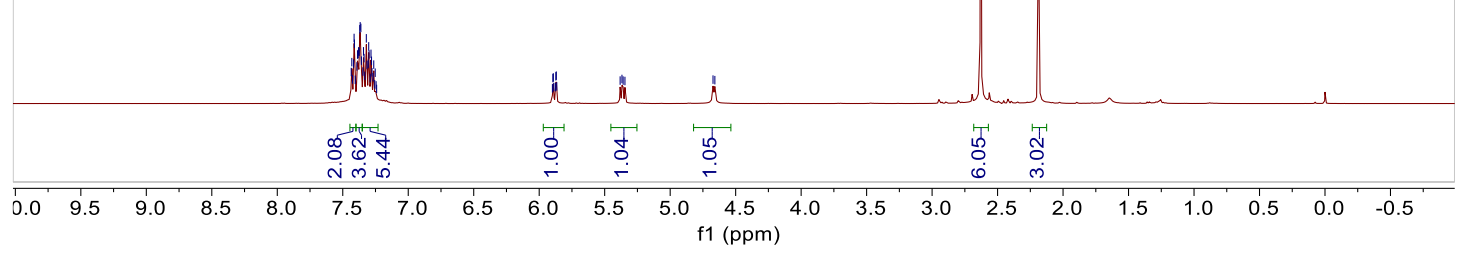

41 


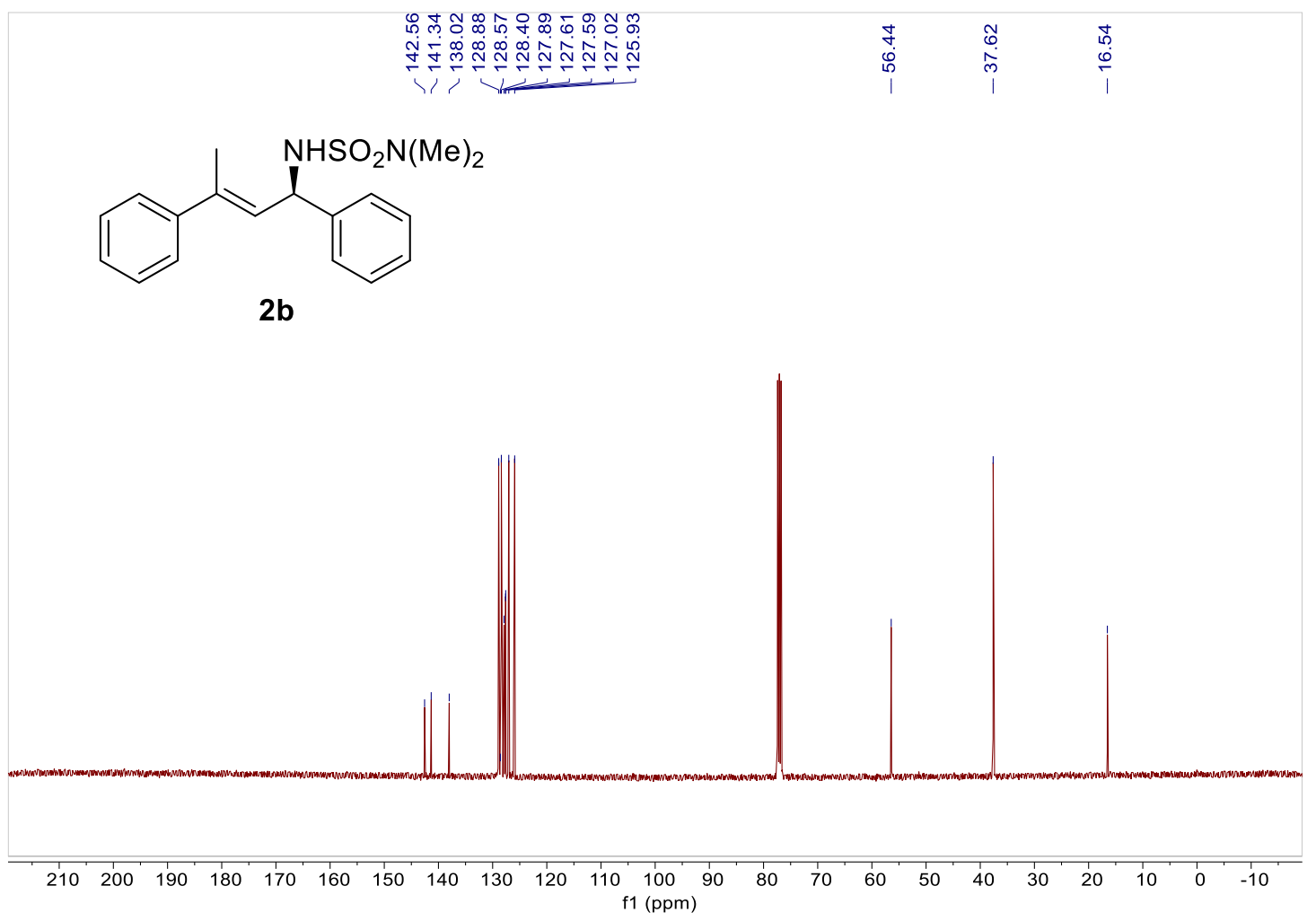

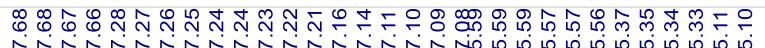

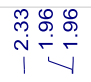<smiles>C/C(=C\[C@H](N)c1ccccc1)c1ccccc1</smiles>

2c

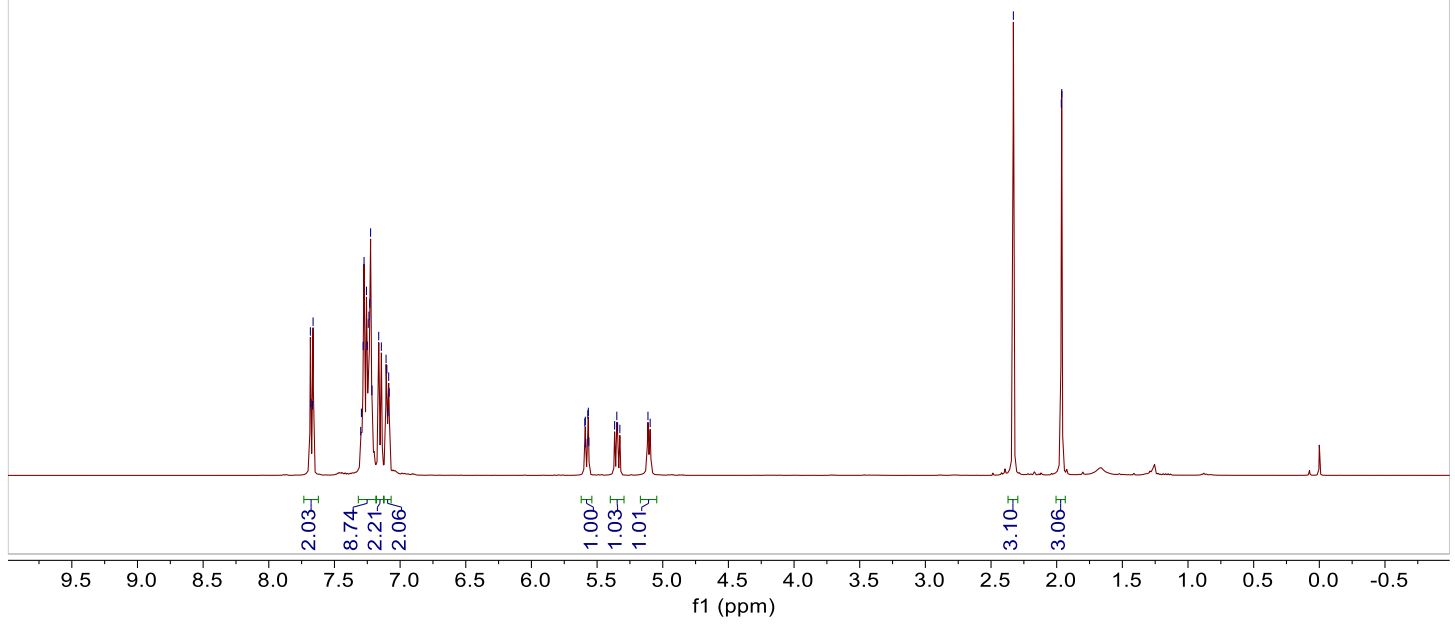

42 


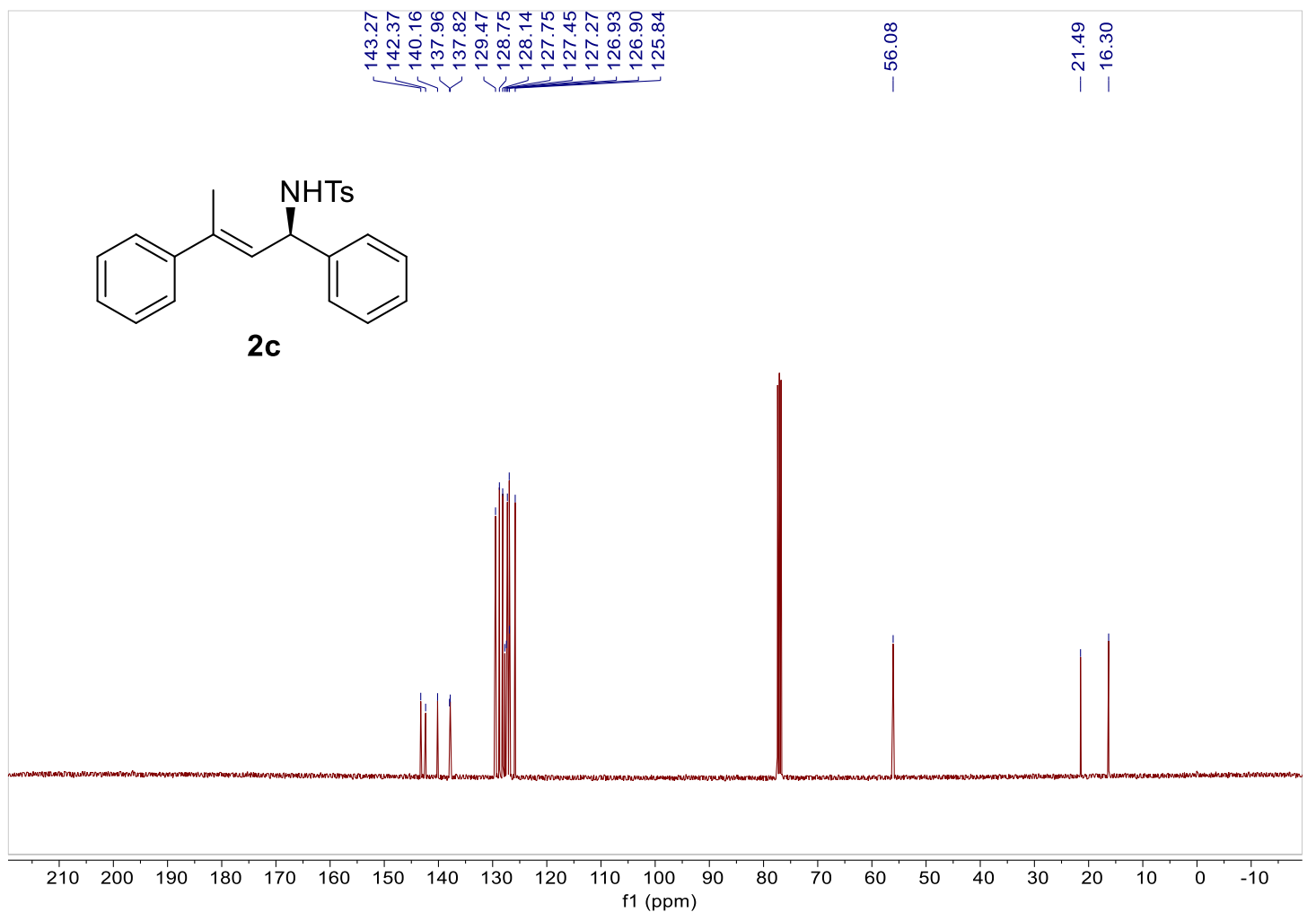

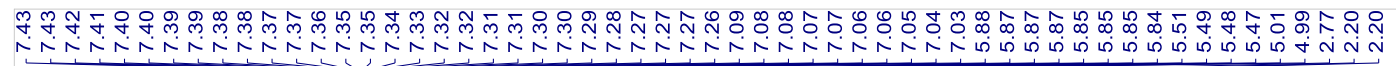<smiles>C/C(=C\[C@H](N)c1ccc(F)cc1)c1ccccc1</smiles>

2d

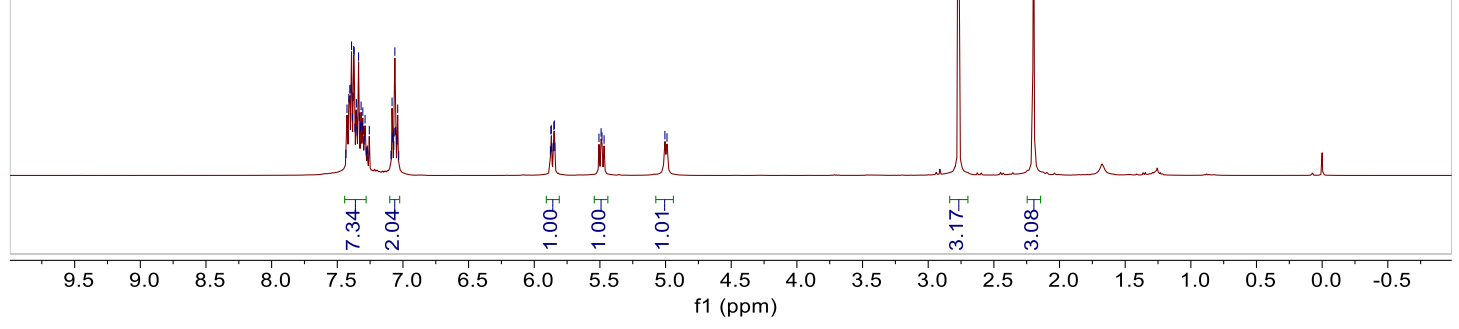

43 


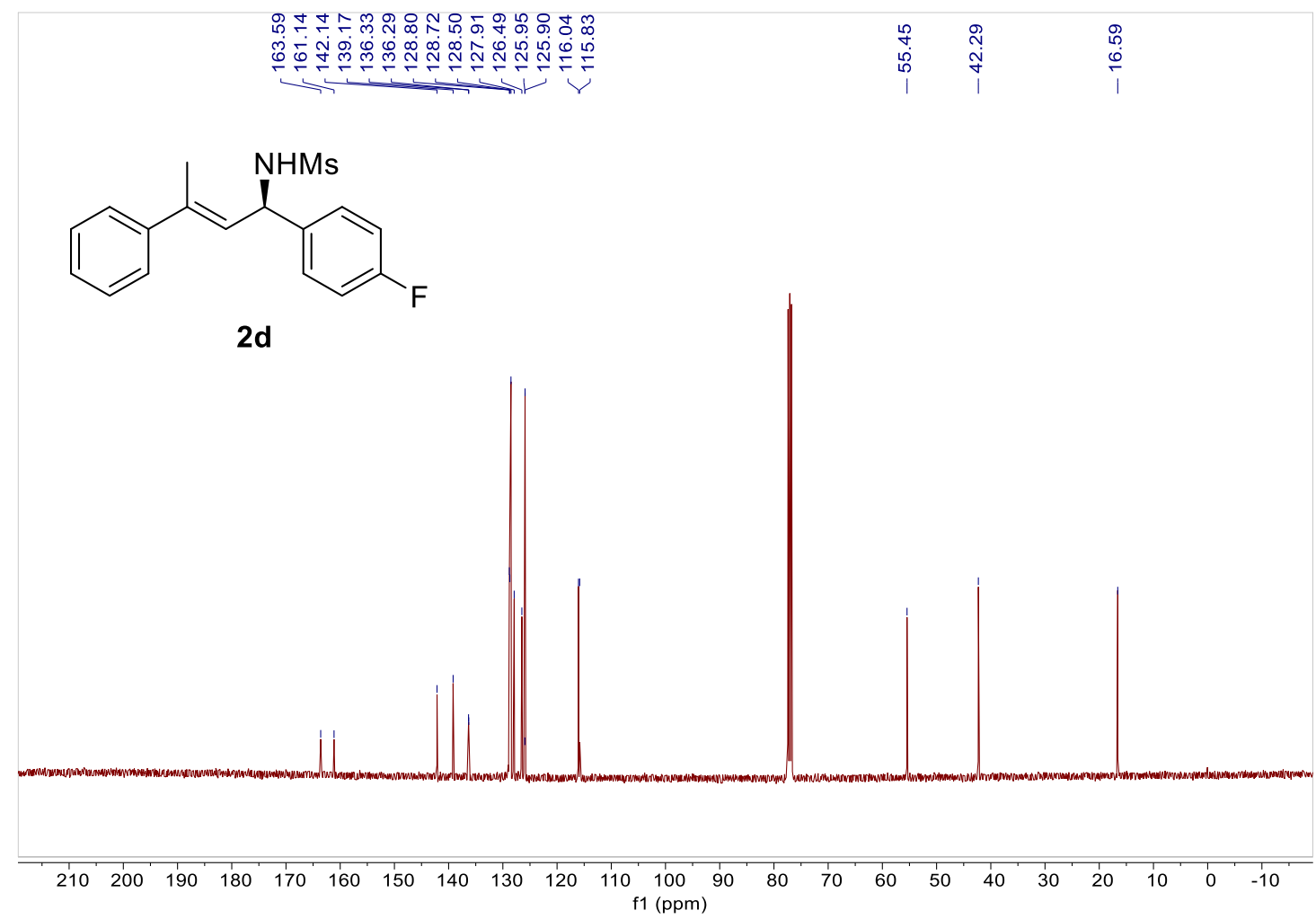

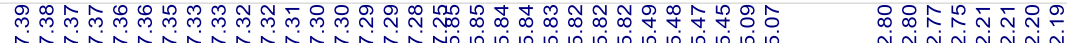

ヘ<smiles>C/C(=C\[C@H](N)c1ccc(Cl)cc1)c1ccccc1</smiles>

$2 e$
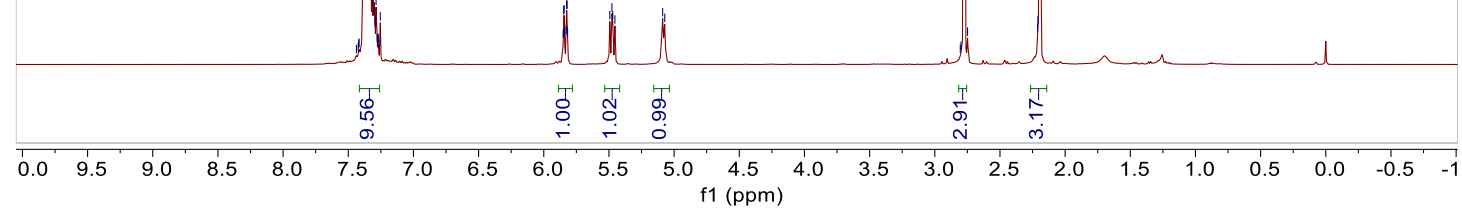

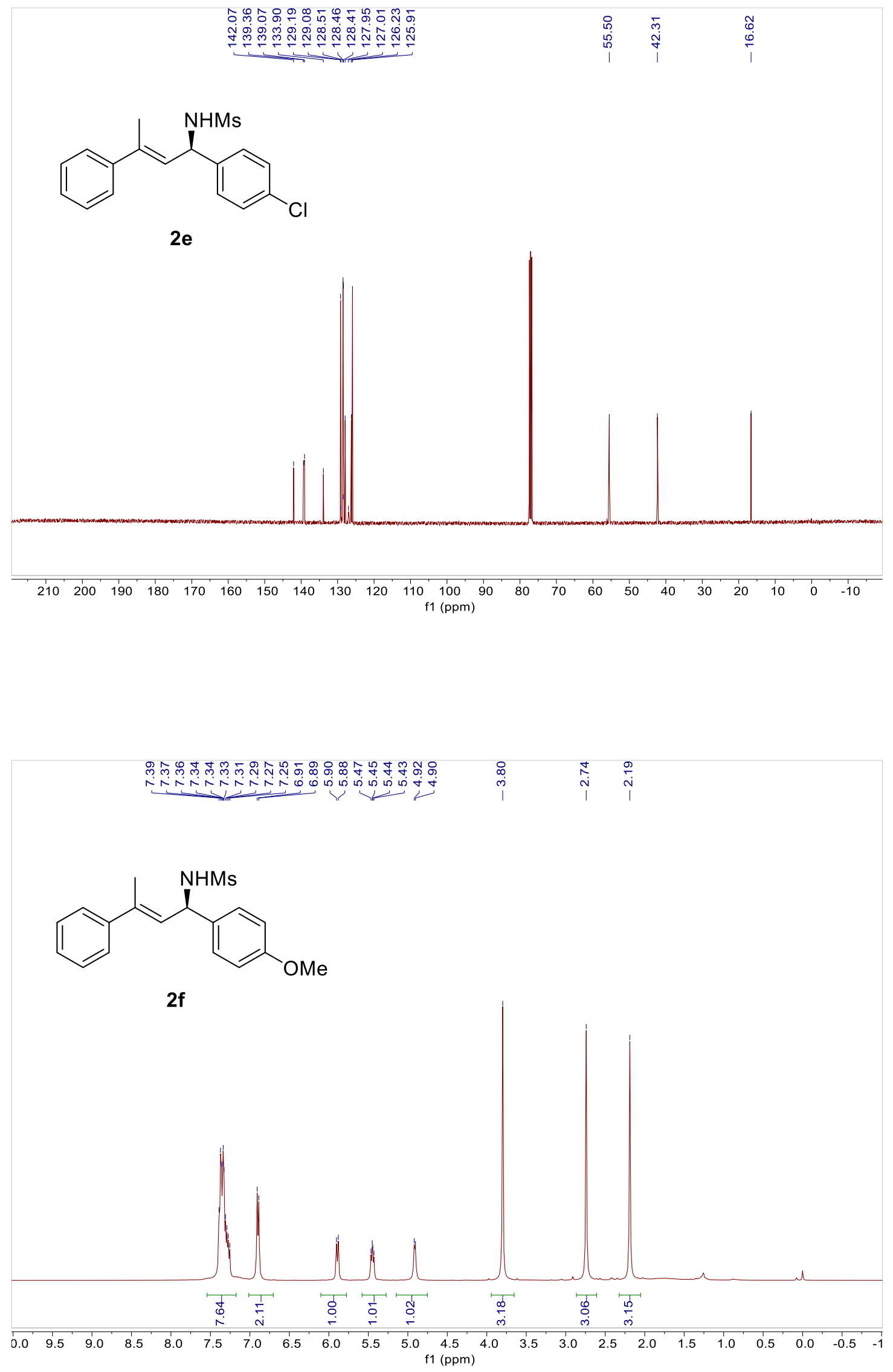

45 


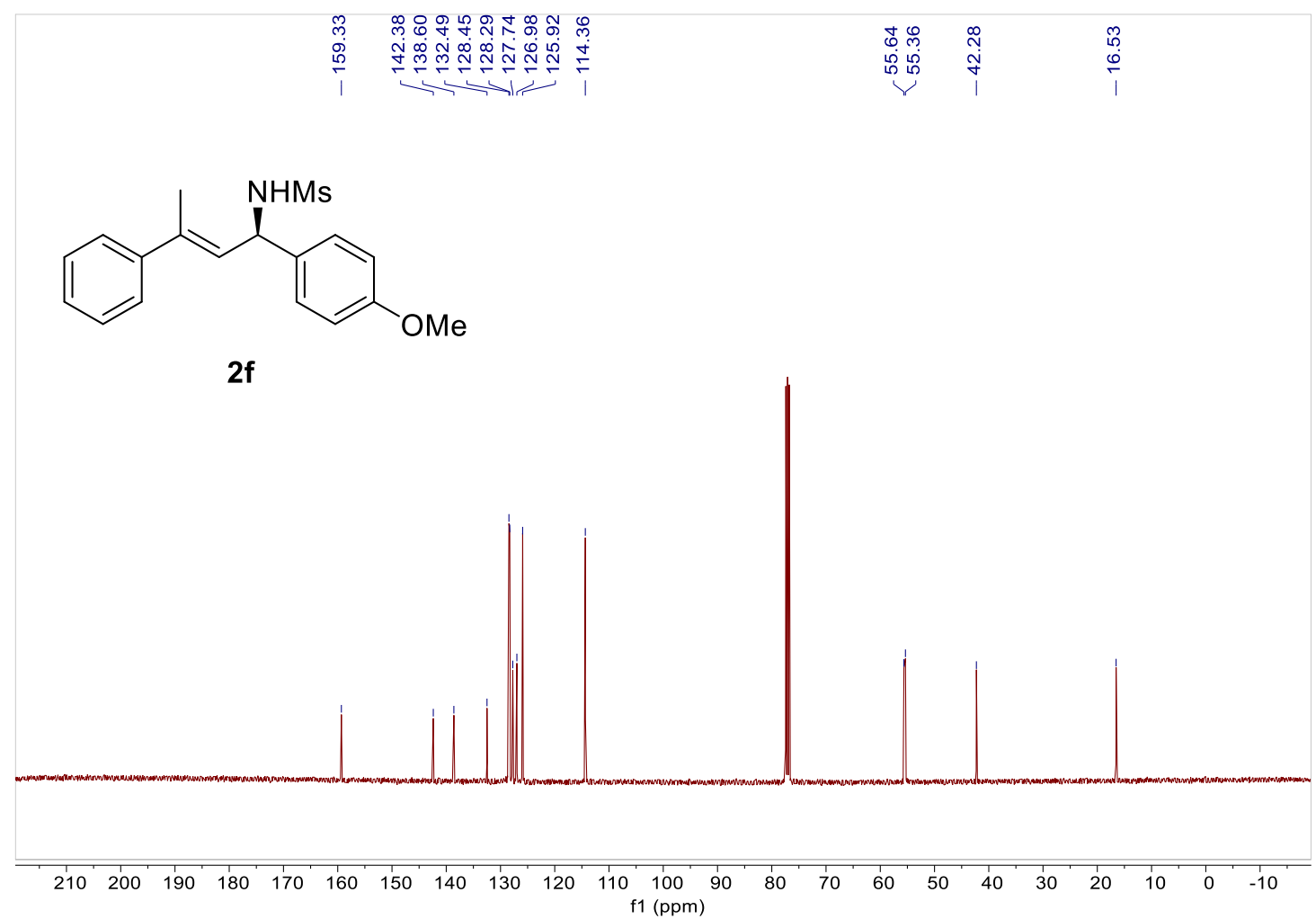

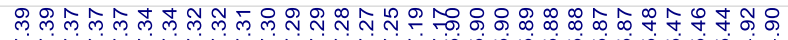

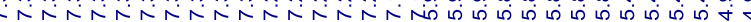

ำ

ำกำ<smiles>C/C(=C\[C@@H](N)c1ccc(C)cc1)c1ccccc1</smiles>

$2 \mathrm{~g}$

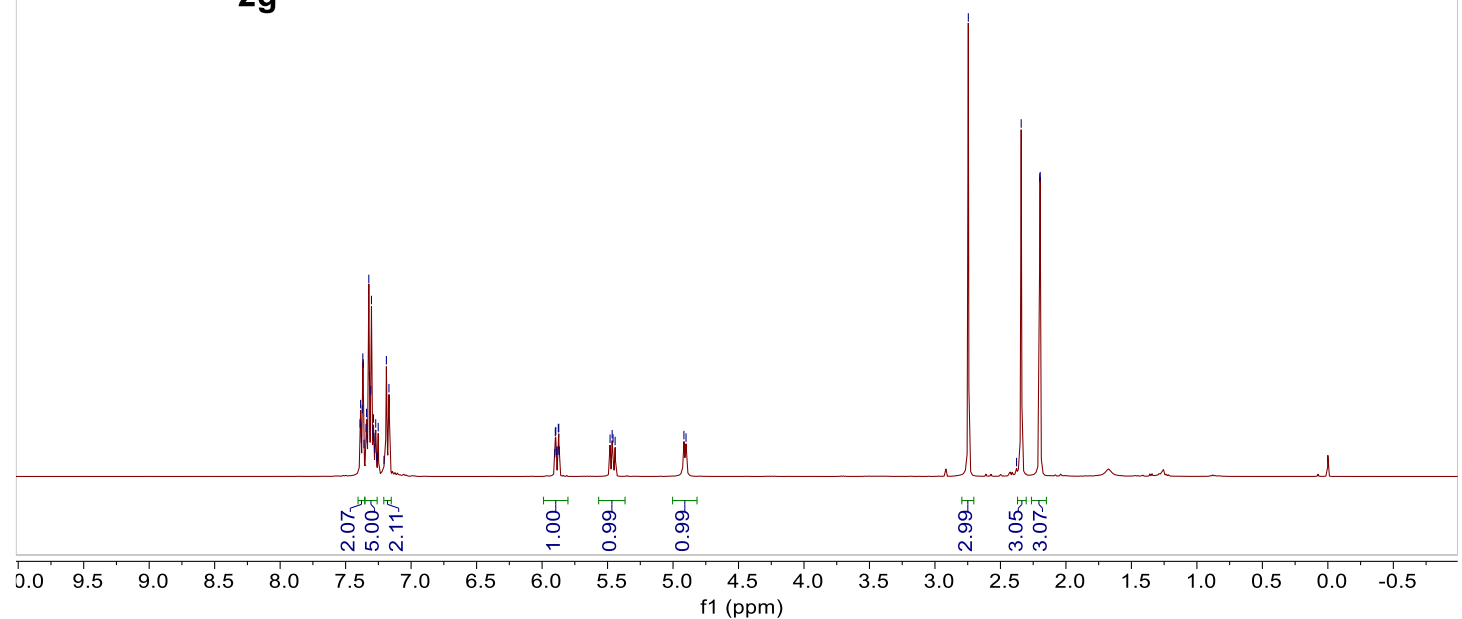




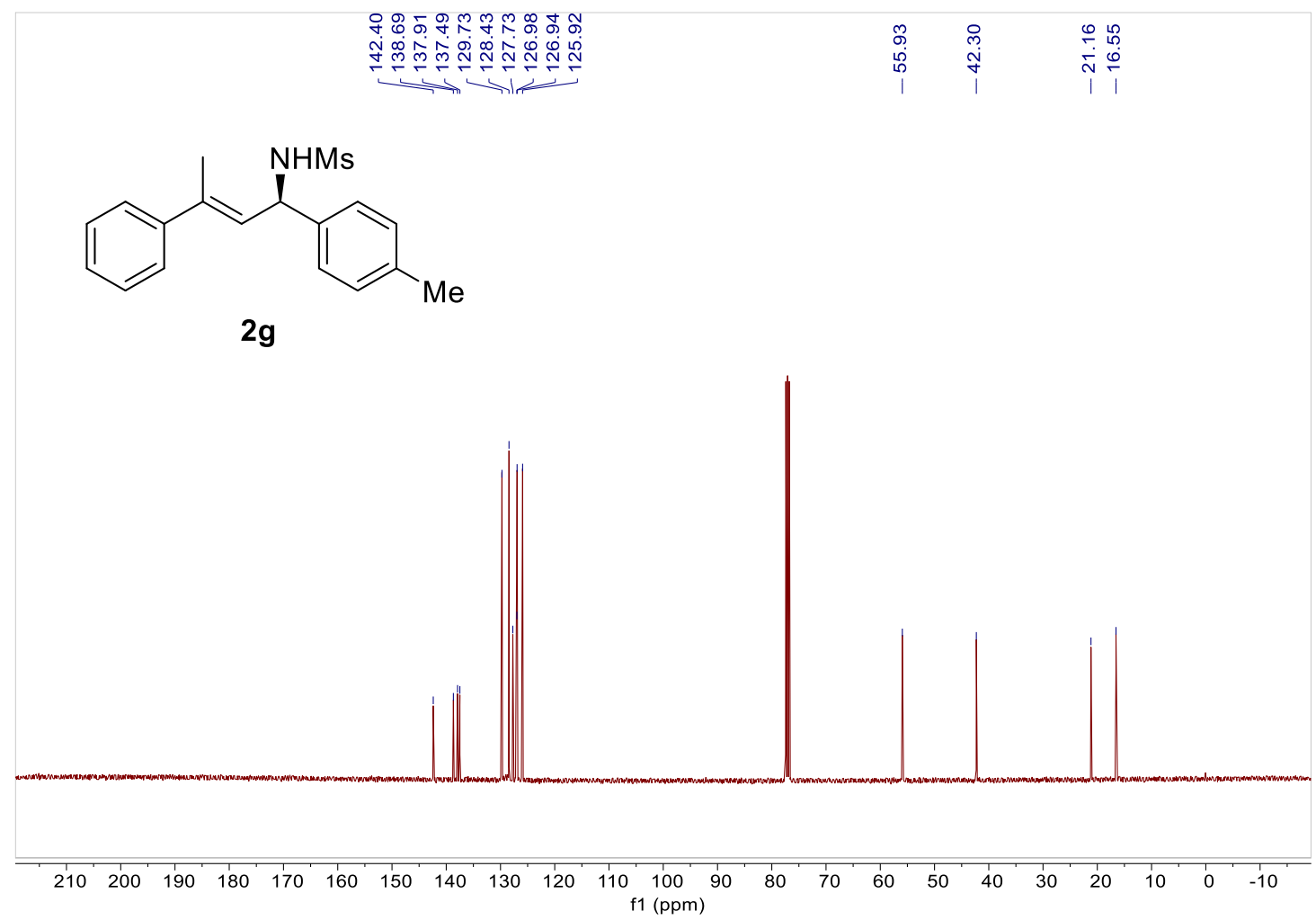

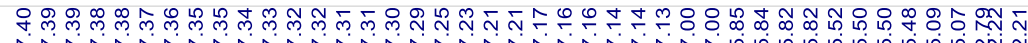

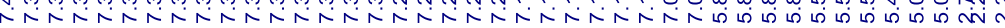<smiles>C/C(=C\[C@H](N)c1cccc(F)c1)c1ccccc1</smiles>

$2 \mathrm{~h}$

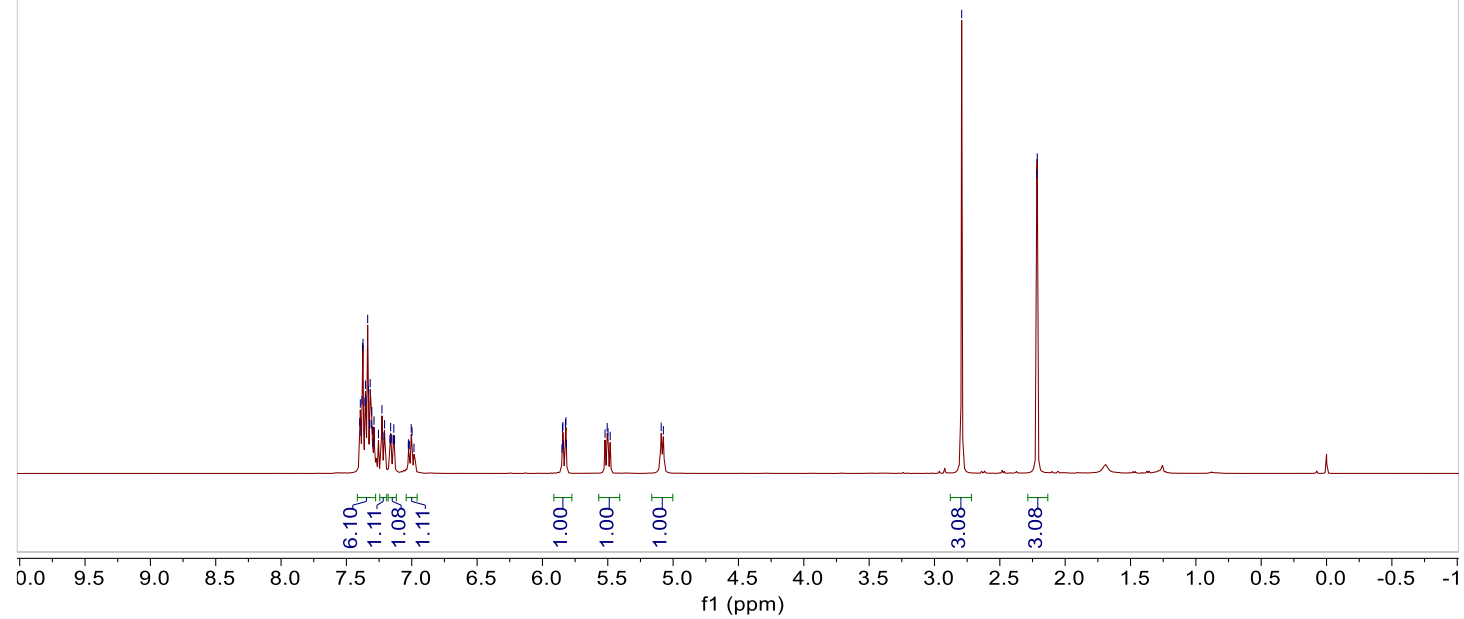




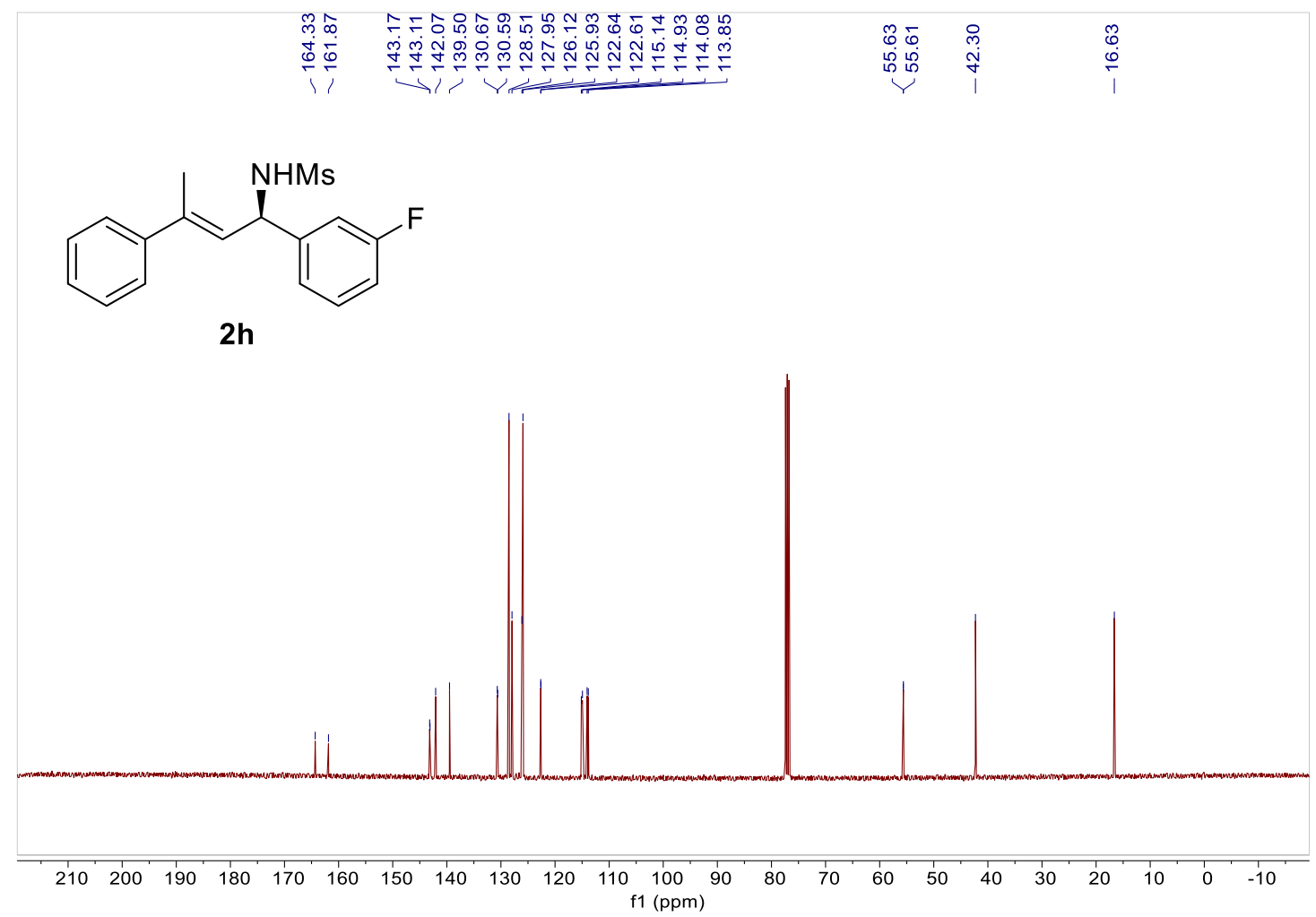

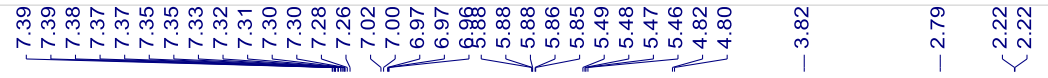<smiles>COc1cccc([C@H](/C=C(\C)c2ccccc2)N(C)C)c1</smiles>

2i

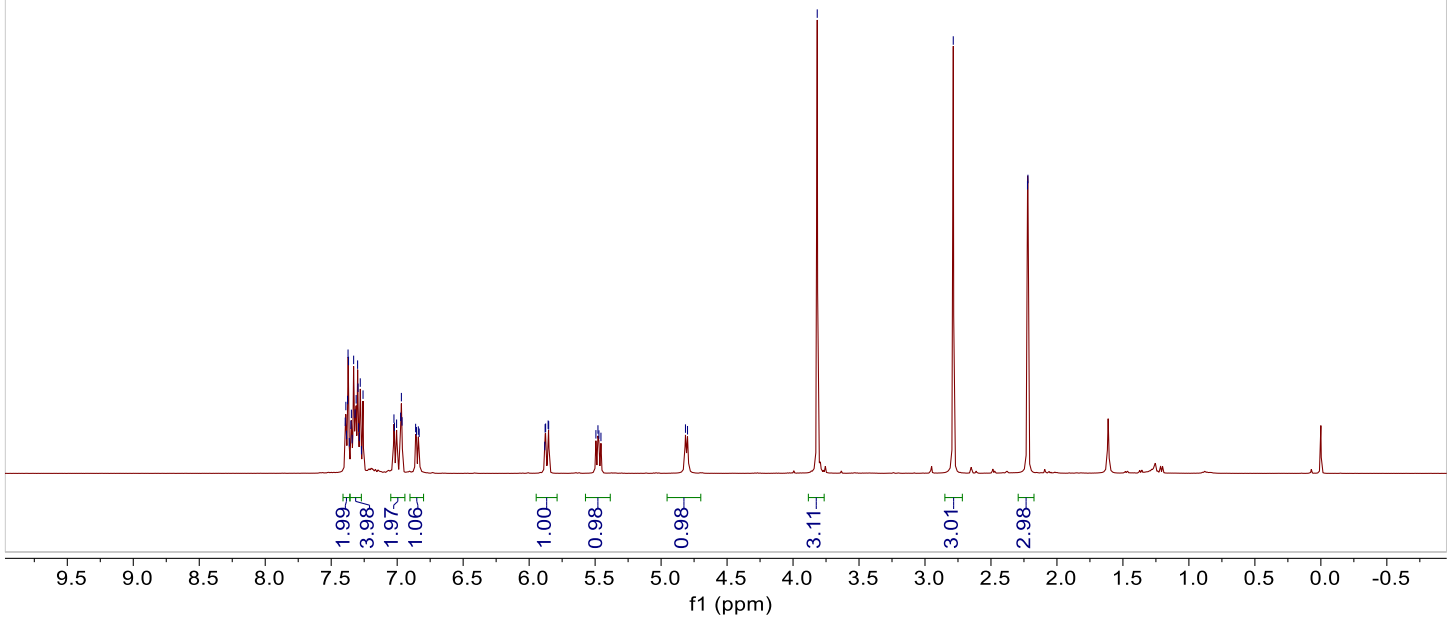




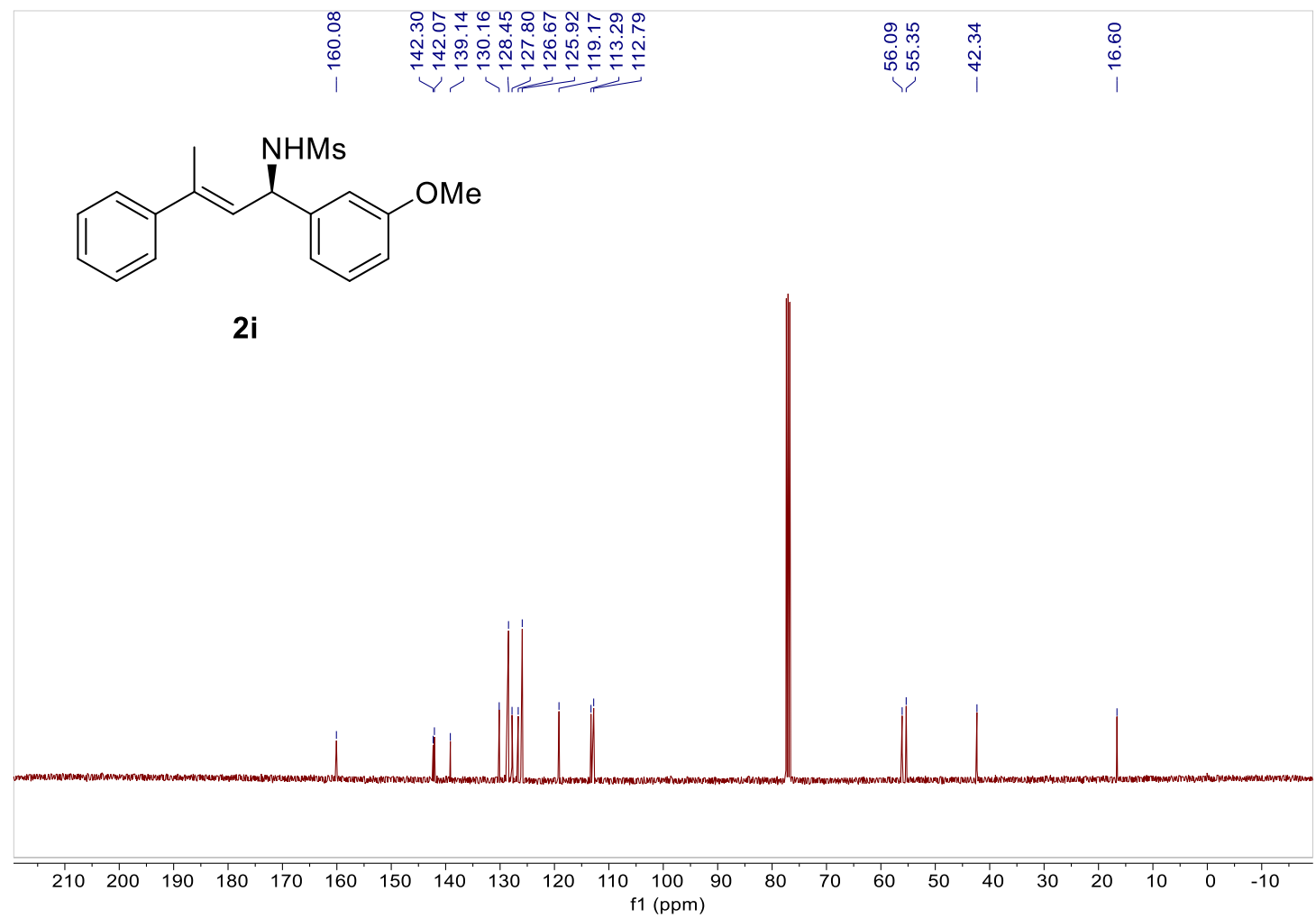

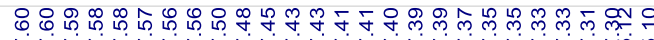

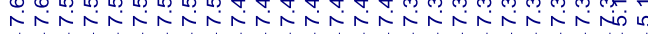

$\stackrel{9}{i} \quad \stackrel{N}{i}$<smiles>C/C(=C\[C@H](N)c1ccccc1P)c1ccccc1</smiles>

2j

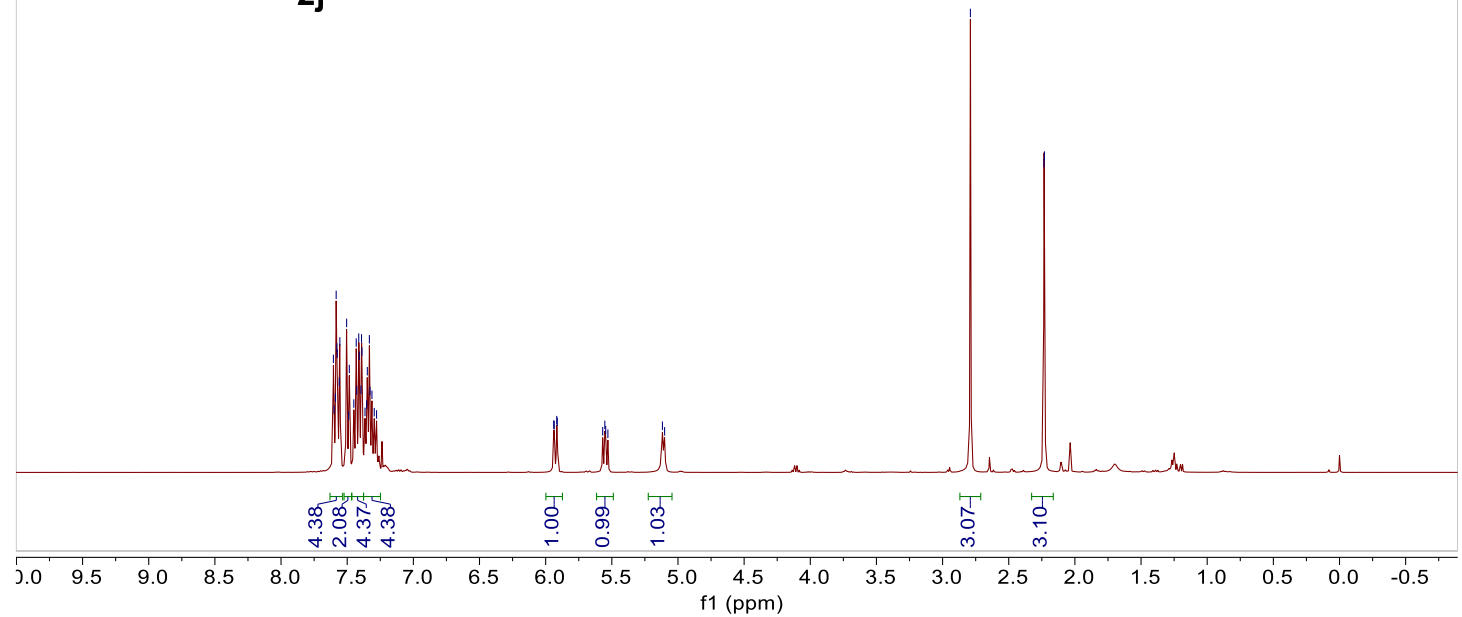



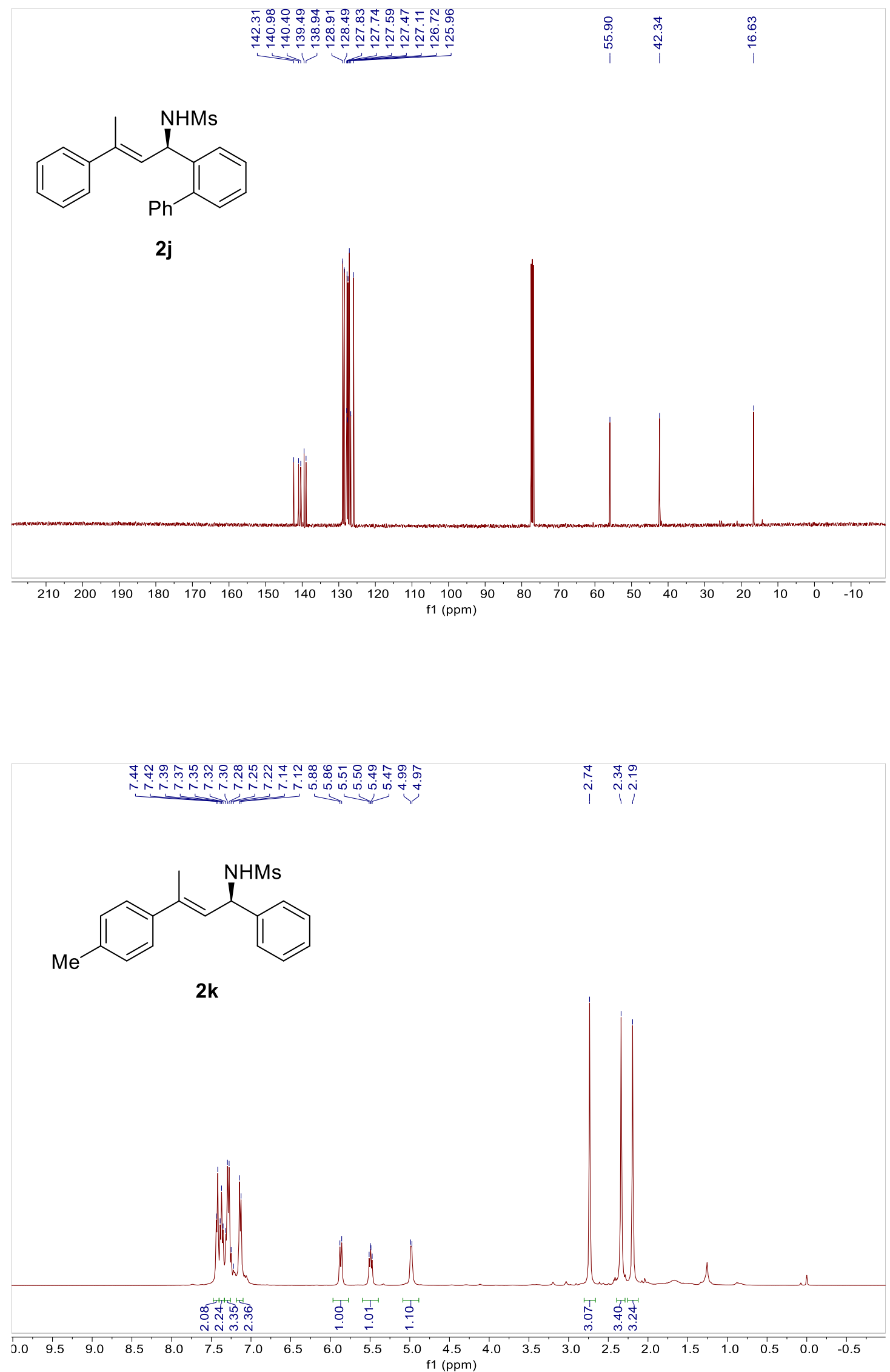

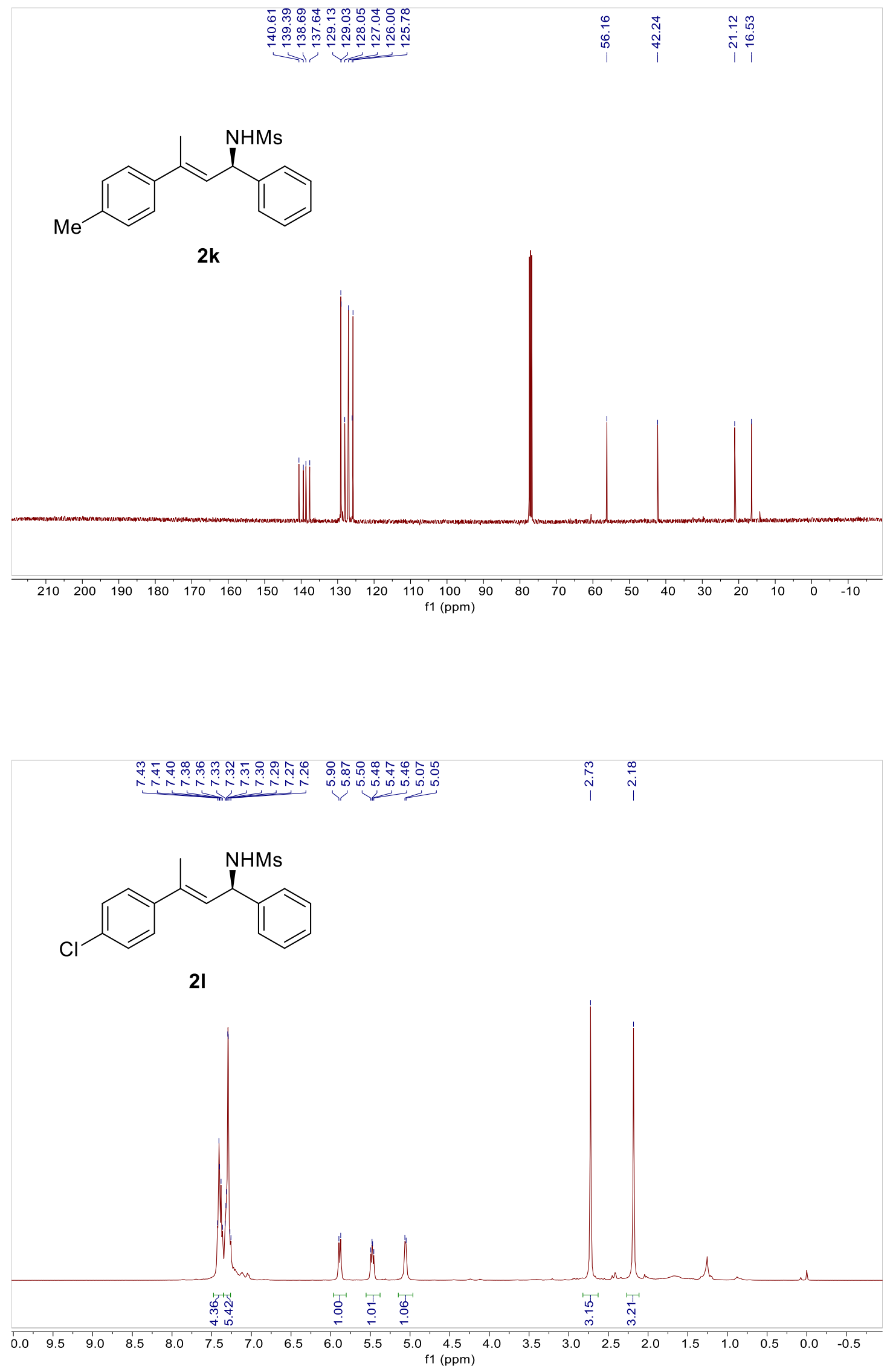

51 


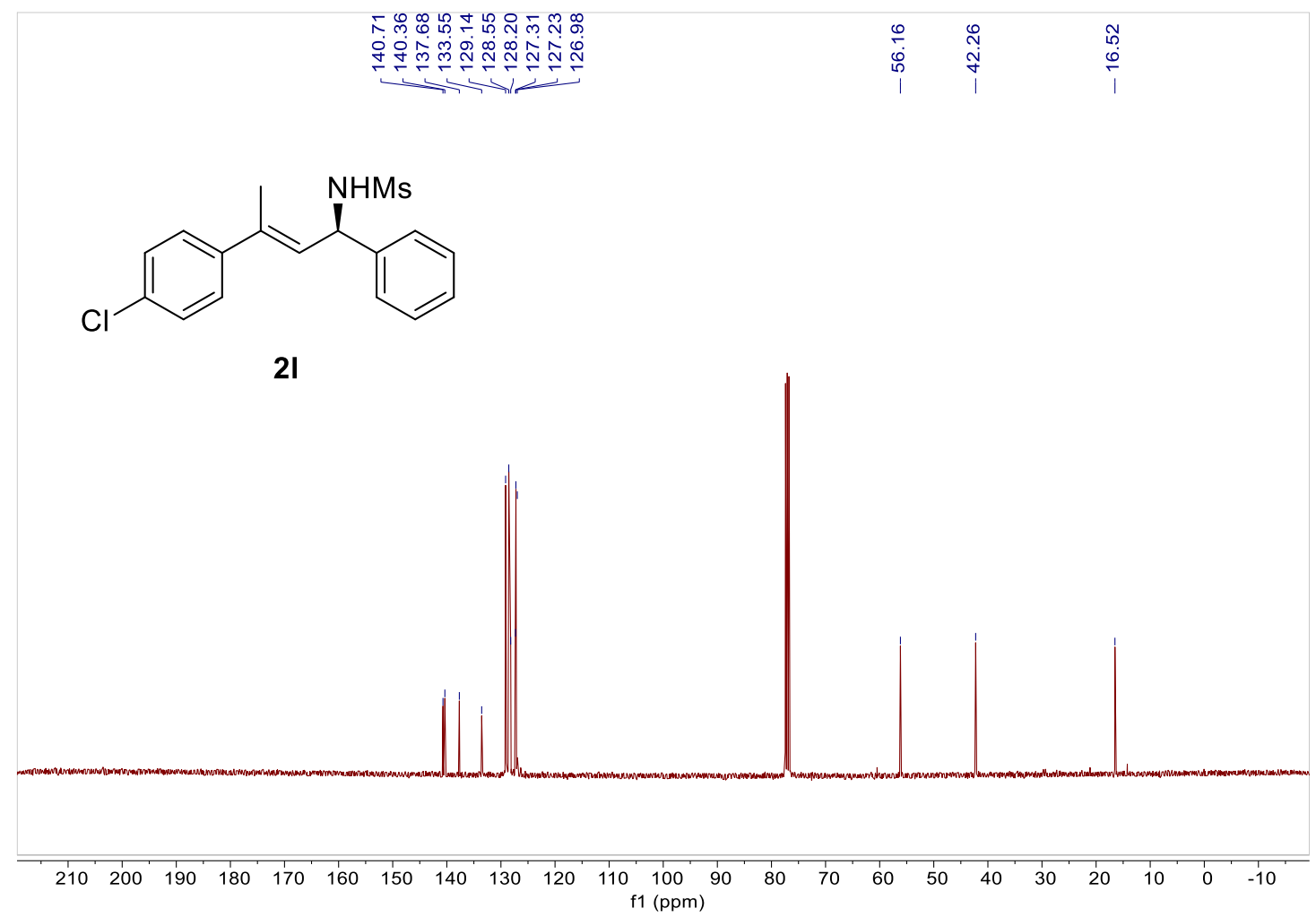

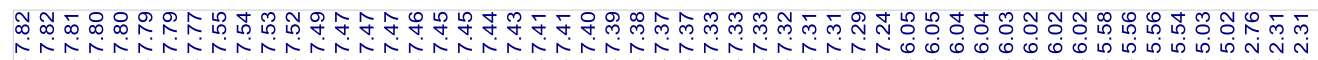<smiles>C/C(=C\[C@H](NC(C)(C)C)c1ccccc1)c1ccc2ccccc2c1</smiles>

$2 m$

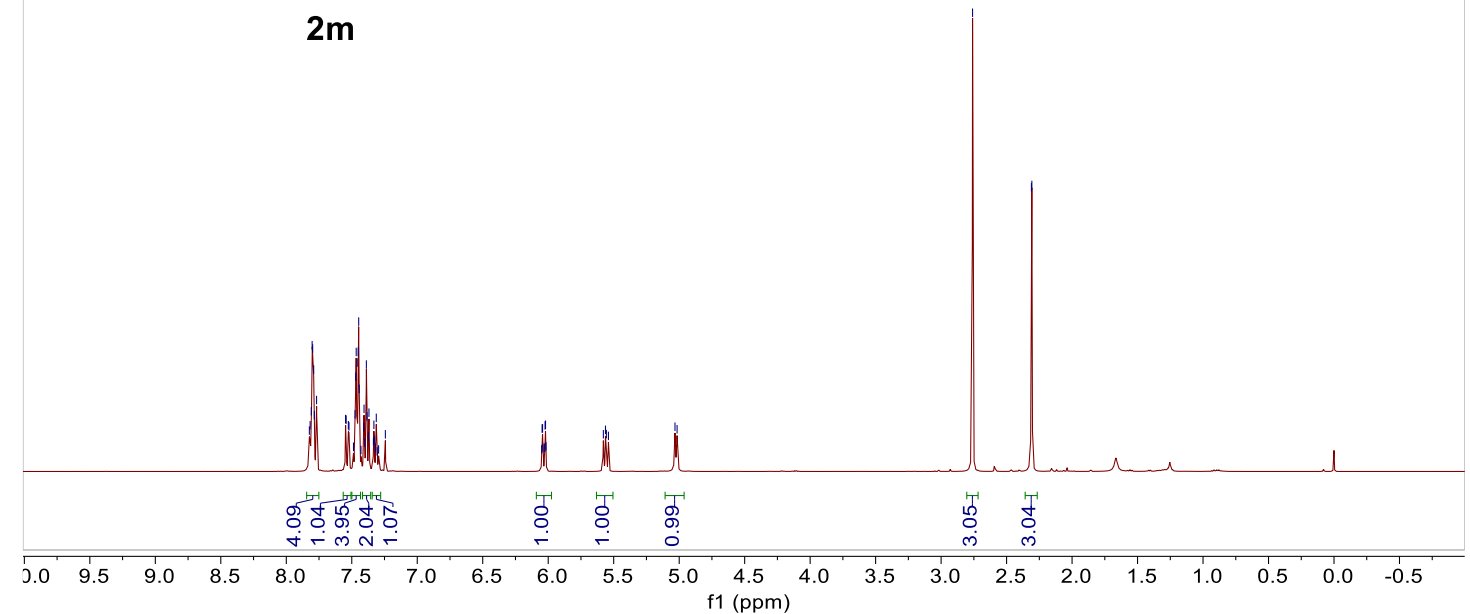




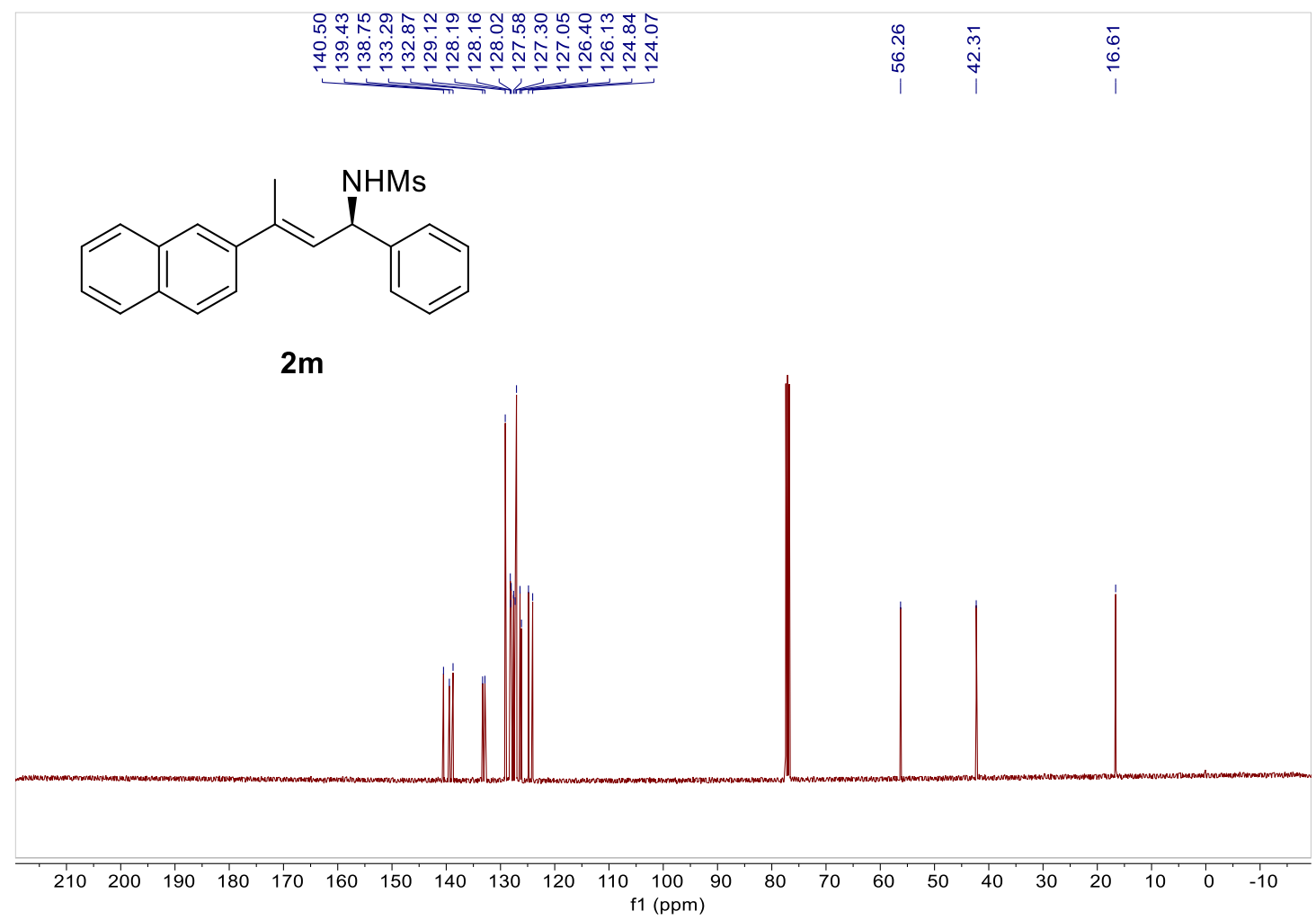

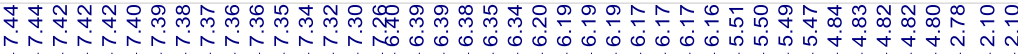<smiles>C/C(=C\[C@@H](c1ccccc1)N(C)C)c1ccco1</smiles>

2n

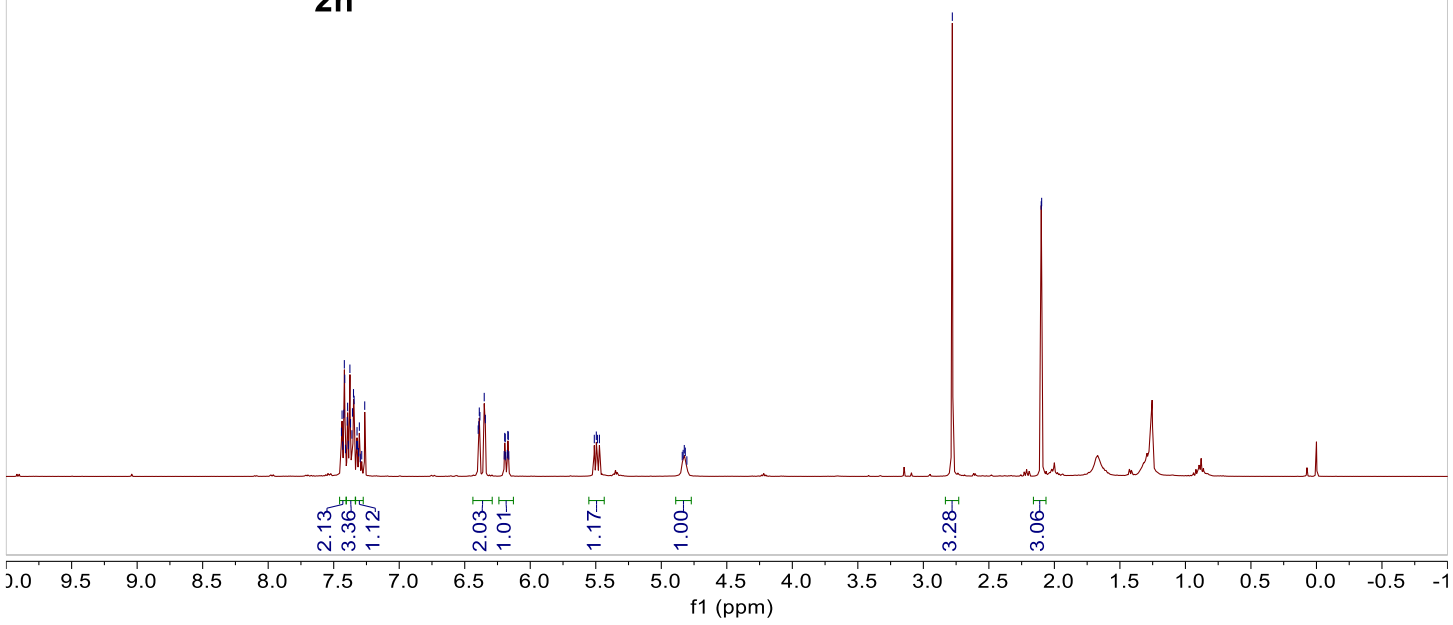



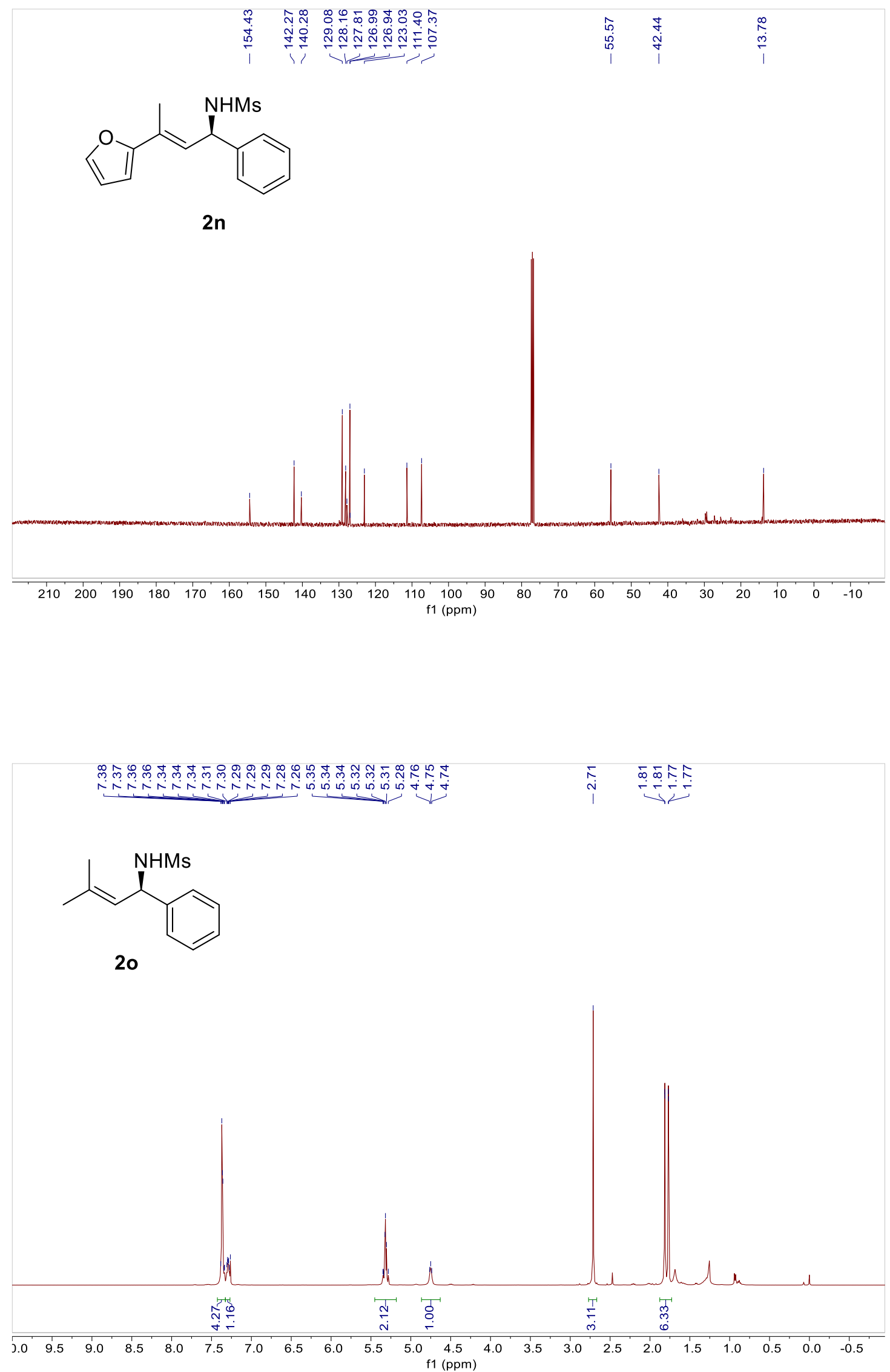


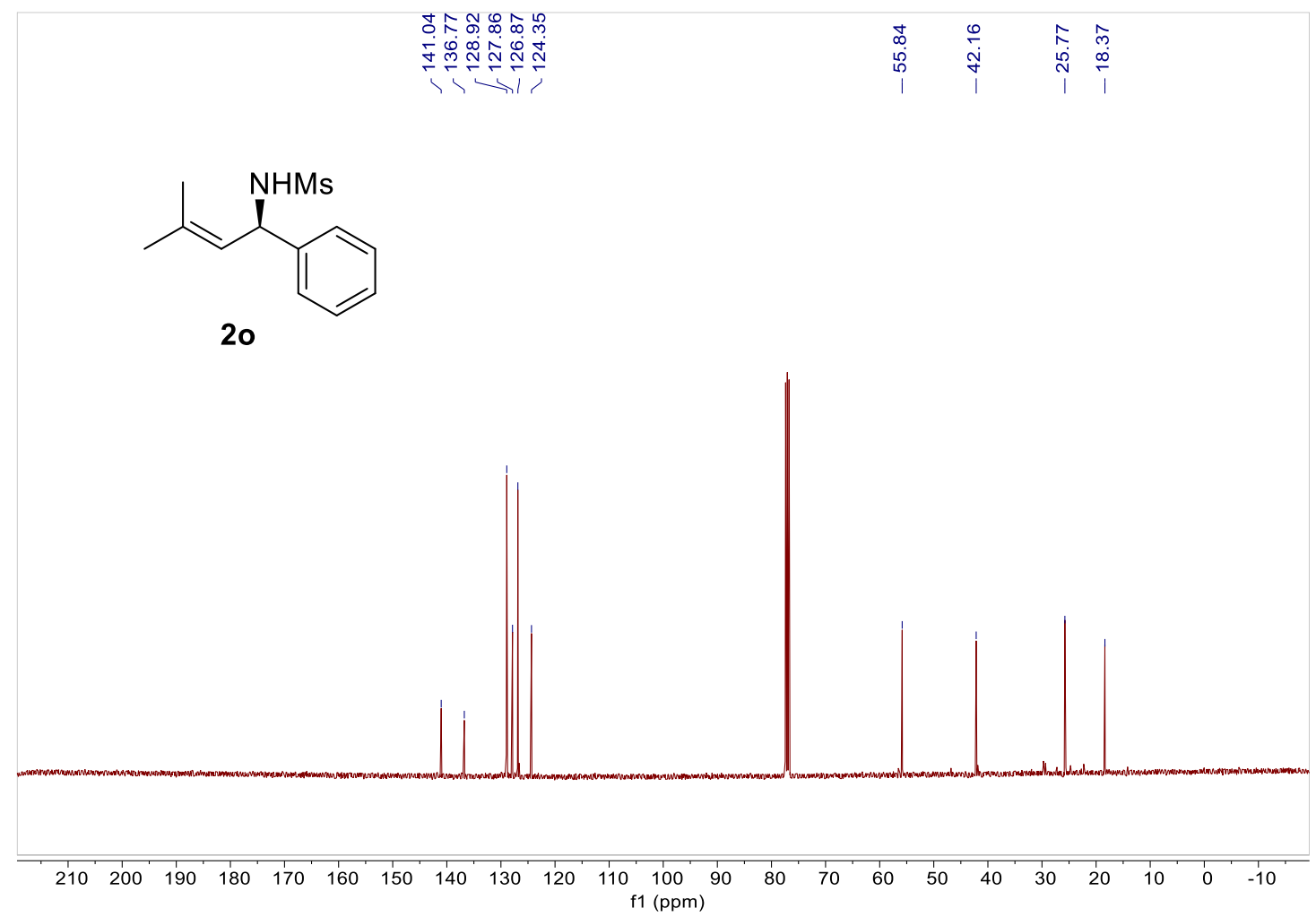

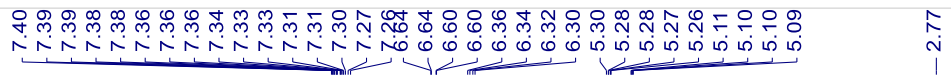<smiles>CNC(/C=C/c1ccccc1)c1ccccc1</smiles>

$2 p$

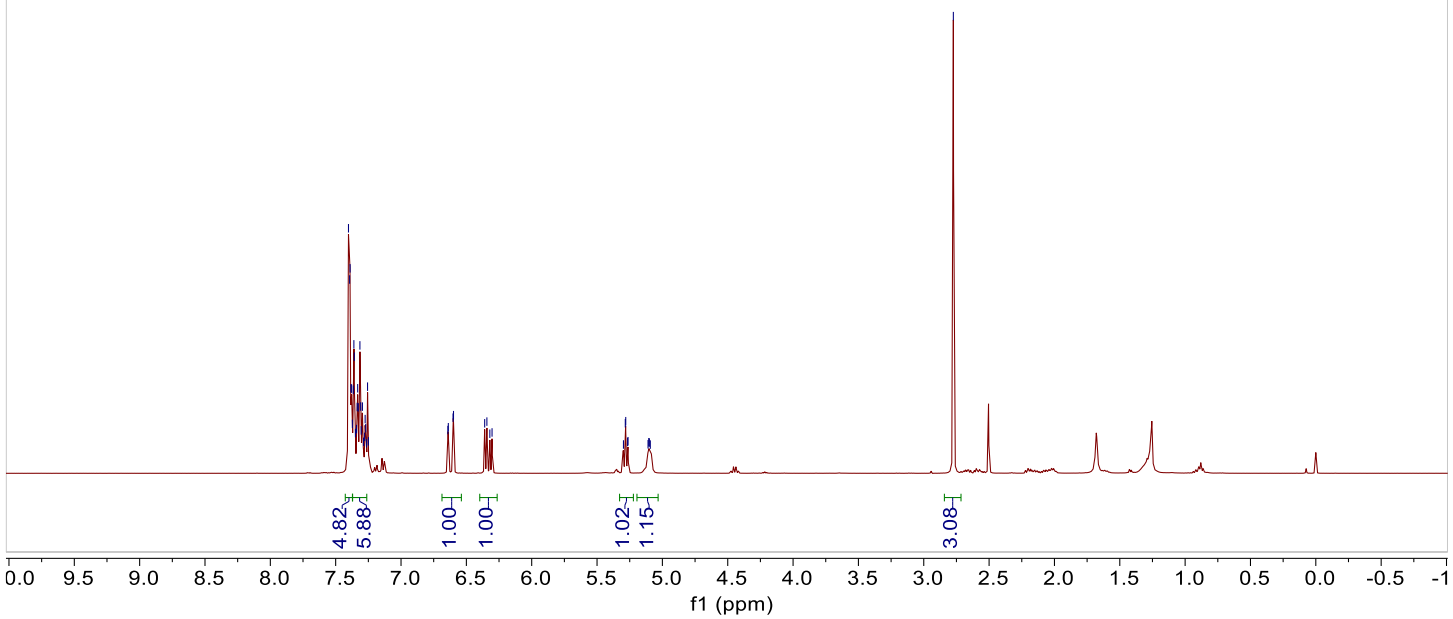




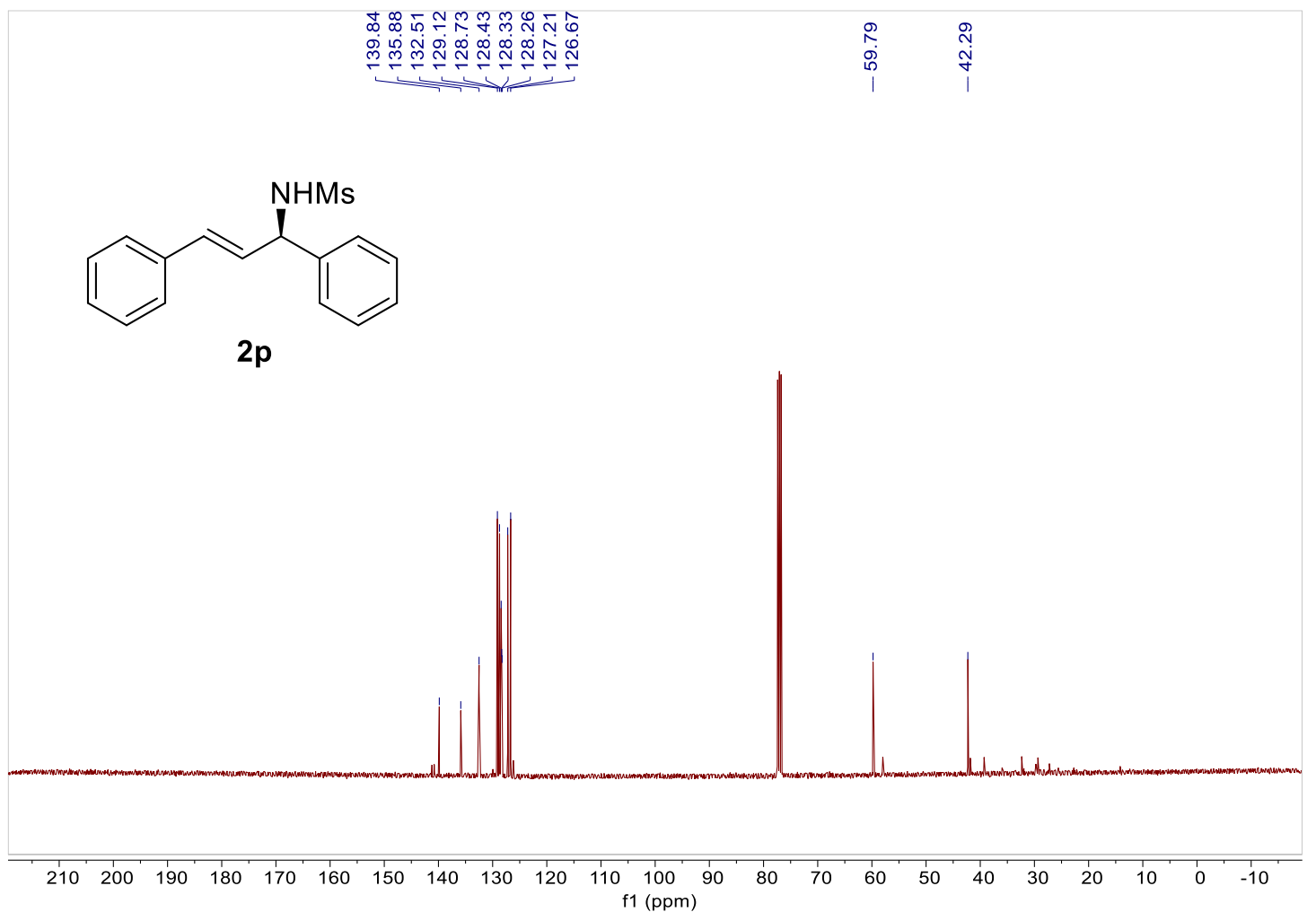

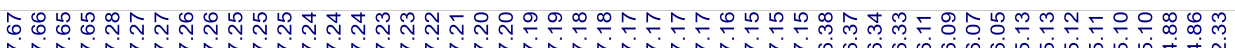<smiles>F[C@H](/C=C/c1ccccc1)c1ccccc1</smiles>

$2 q$

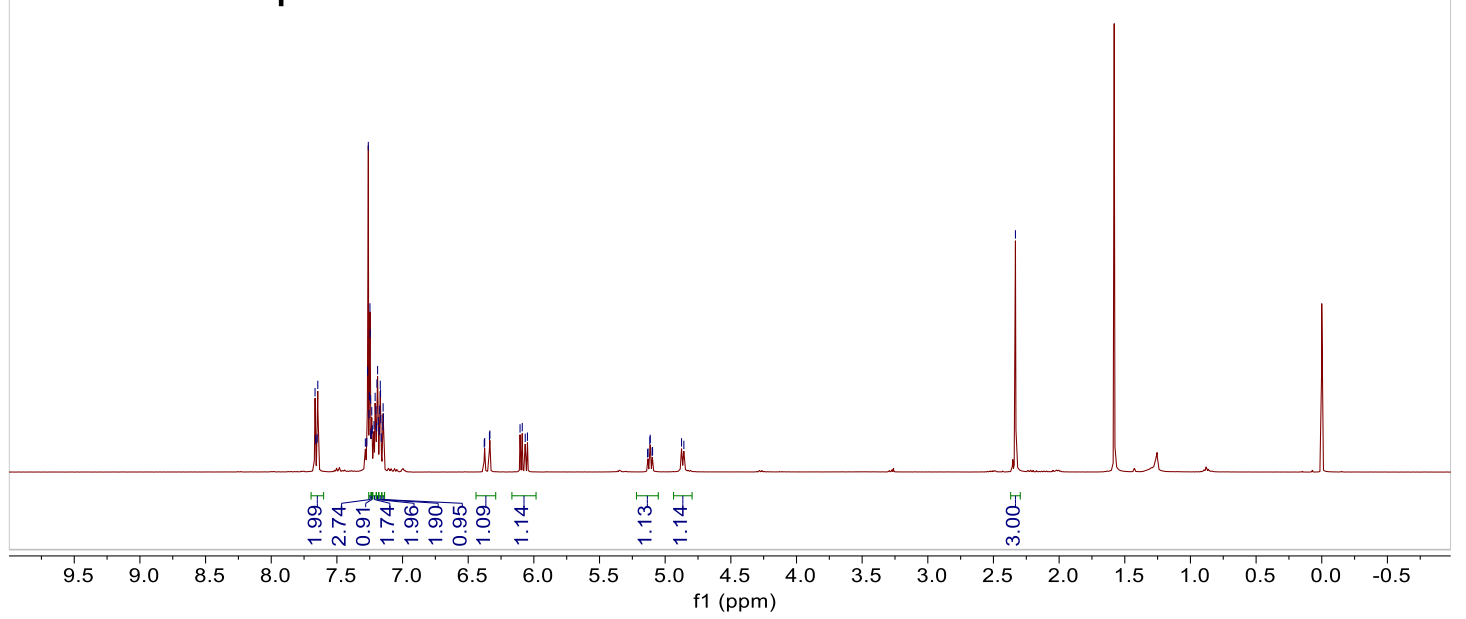

56 


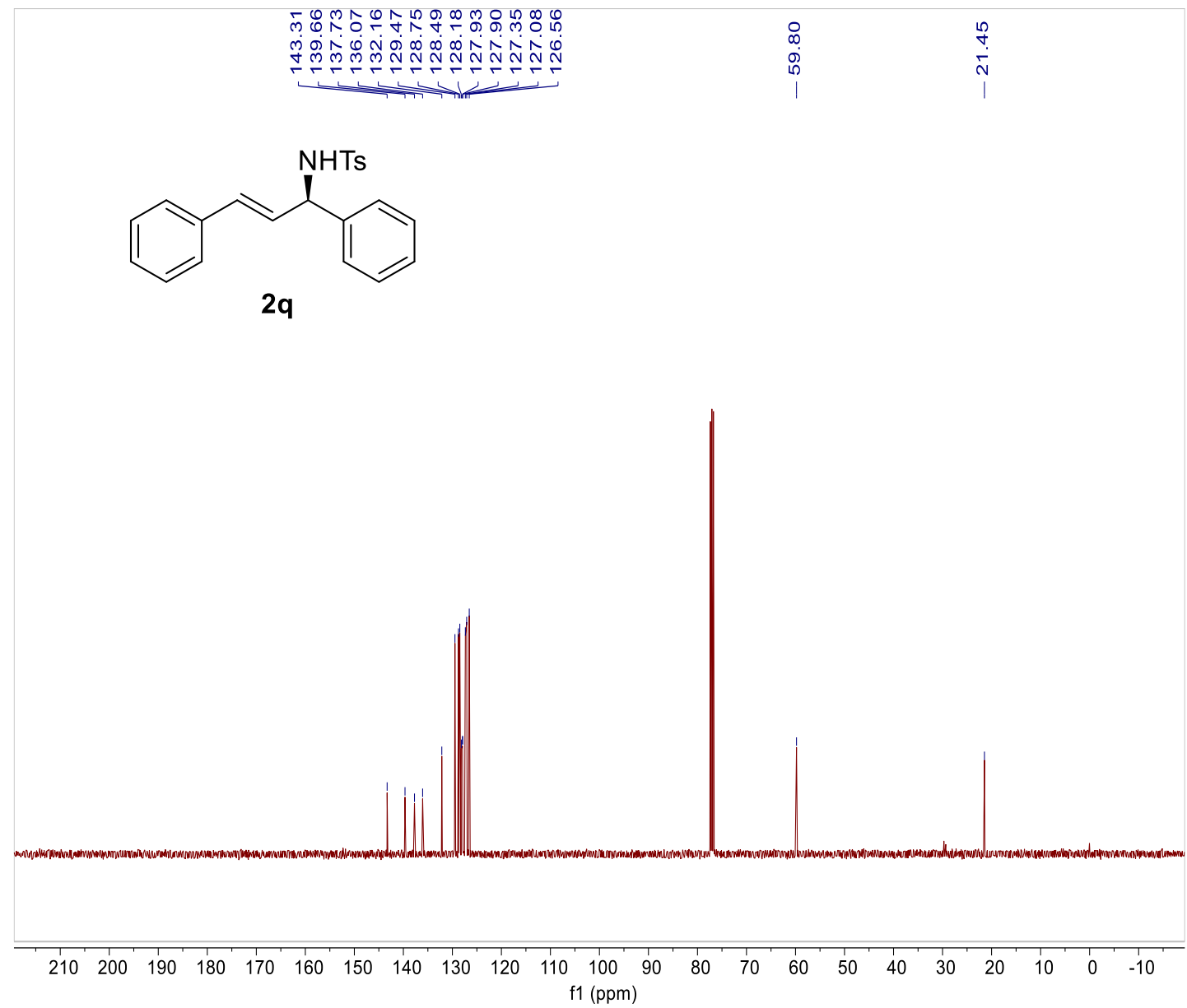




\section{HPLC spectra}

Data File D: \DATA $\backslash$ LWD $\backslash L W D-5-14-1 \backslash L$ WD-5-14-1 2019-03-13 10-06-46\015-3201.D Sample Name: ZX-3-81-Ms-RAC

$\begin{array}{lr}\text { Acq. Operator : } & \text { Seq. Line : } 32 \\ \text { Acq. Instrument : Instrument } 1 & \text { Location : Vial } 15 \\ \text { Injection Date : } 3 / 14 / 20192: 03: 35 \mathrm{AM} & \text { Inj : } 1\end{array}$

Acq. Method : D: \DATA $\backslash$ LWD $\backslash L$ LDD-5-14-1 \LWD-5-14-1 2019-03-13 10-06-46 VWD-AD (1-2)-90-10-1ML $-3 \mathrm{UL}-254 \mathrm{NM}-40 \mathrm{MIN} . \mathrm{M}$

Last changed : 3/13/2019 12:56:36 PM

AnalYsis Method : D: \NETHOD ZX\DAD-OD-(1-2)-85-15-1ML-3UL-ĂLL-60MIN.M

Last changed : 4/13/2019 1:01:10 PM

(modified after loading)

Additional Info : Peak (s) manually integrated

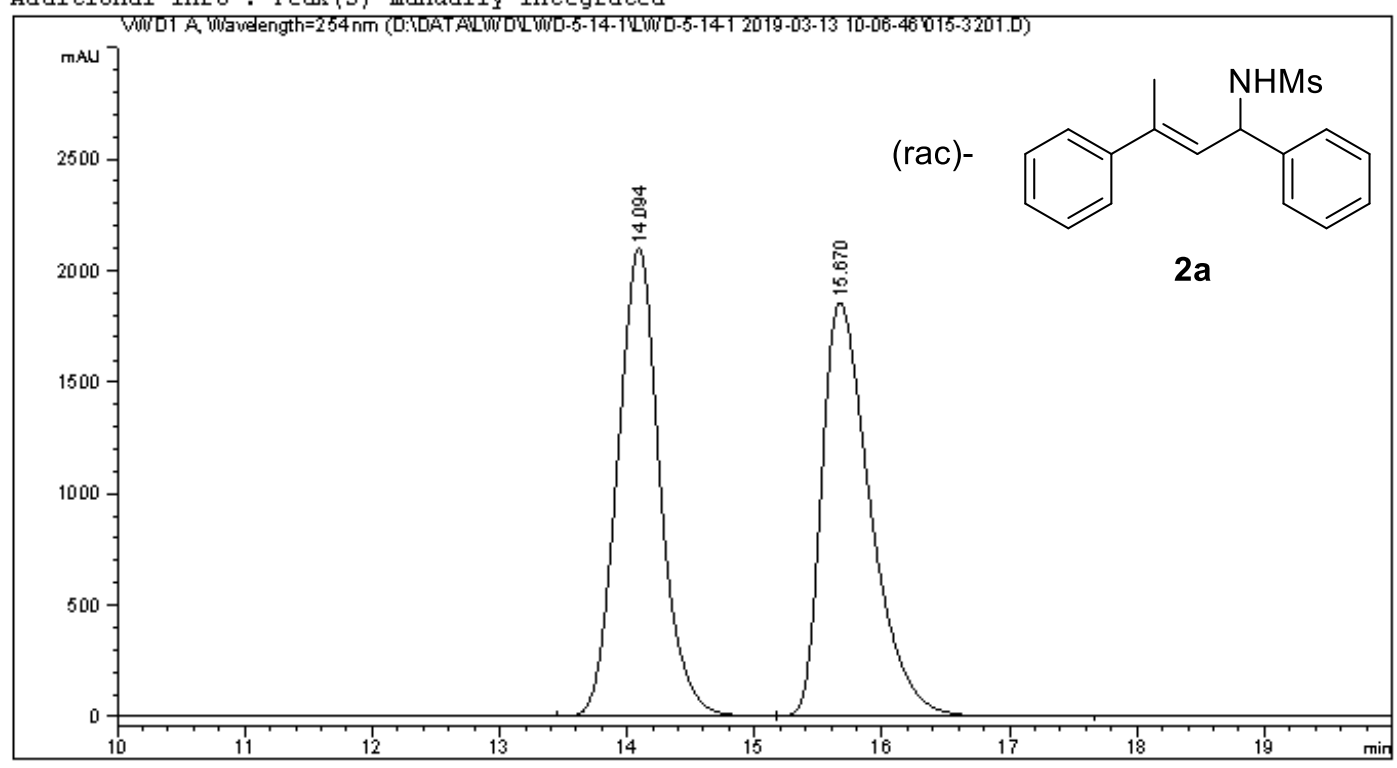

Area Percent Report

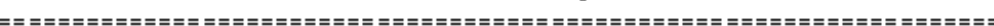

$\begin{array}{lll}\text { Sorted By } & : & \text { Signal } \\ \text { Multiplier } & : & 1.0000 \\ \text { Dilution } & : & 1.0000\end{array}$

Use Multiplier \& Dilution Factor with IsTDs

Signal 1: VWD $\mathrm{A}$, Wavelength=254 nm

\begin{tabular}{|c|c|c|c|c|c|}
\hline $\begin{array}{c}\text { Peak } \\
\#\end{array}$ & $\begin{array}{l}\text { RetTime Type } \\
\text { [min] }\end{array}$ & $\begin{array}{l}\text { Width } \\
\text { [min] }\end{array}$ & $\begin{array}{c}\text { Àrea } \\
\text { [mÂt*s] }\end{array}$ & $\begin{array}{l}\text { Height } \\
\text { [mind] }\end{array}$ & $\begin{array}{c}\text { Area } \\
\stackrel{4}{5}\end{array}$ \\
\hline--- & --- & -------1 & |--------- & |---------- | & ------- \\
\hline 1 & $14.094 \mathrm{BB}$ & 0.3512 & 4. $84024 \mathrm{e} 4$ & 2097.80518 & 49.7409 \\
\hline 2 & $15.670 \mathrm{BB}$ & 0.4040 & $4.89068 \mathrm{e} 4$ & 1852.25452 & 50.2591 \\
\hline \multicolumn{3}{|c|}{ Totals : } & $9.73092 \mathrm{e} 4$ & 3950.05969 & \\
\hline
\end{tabular}


Data File D: \DATA $\backslash L D \backslash L W D-5-14-1 \backslash L$ LD-5-14-1 2019-03-13 10-06-46\016-3301.D

Sample Name: $2 X-3-83-\mathrm{Ms}$
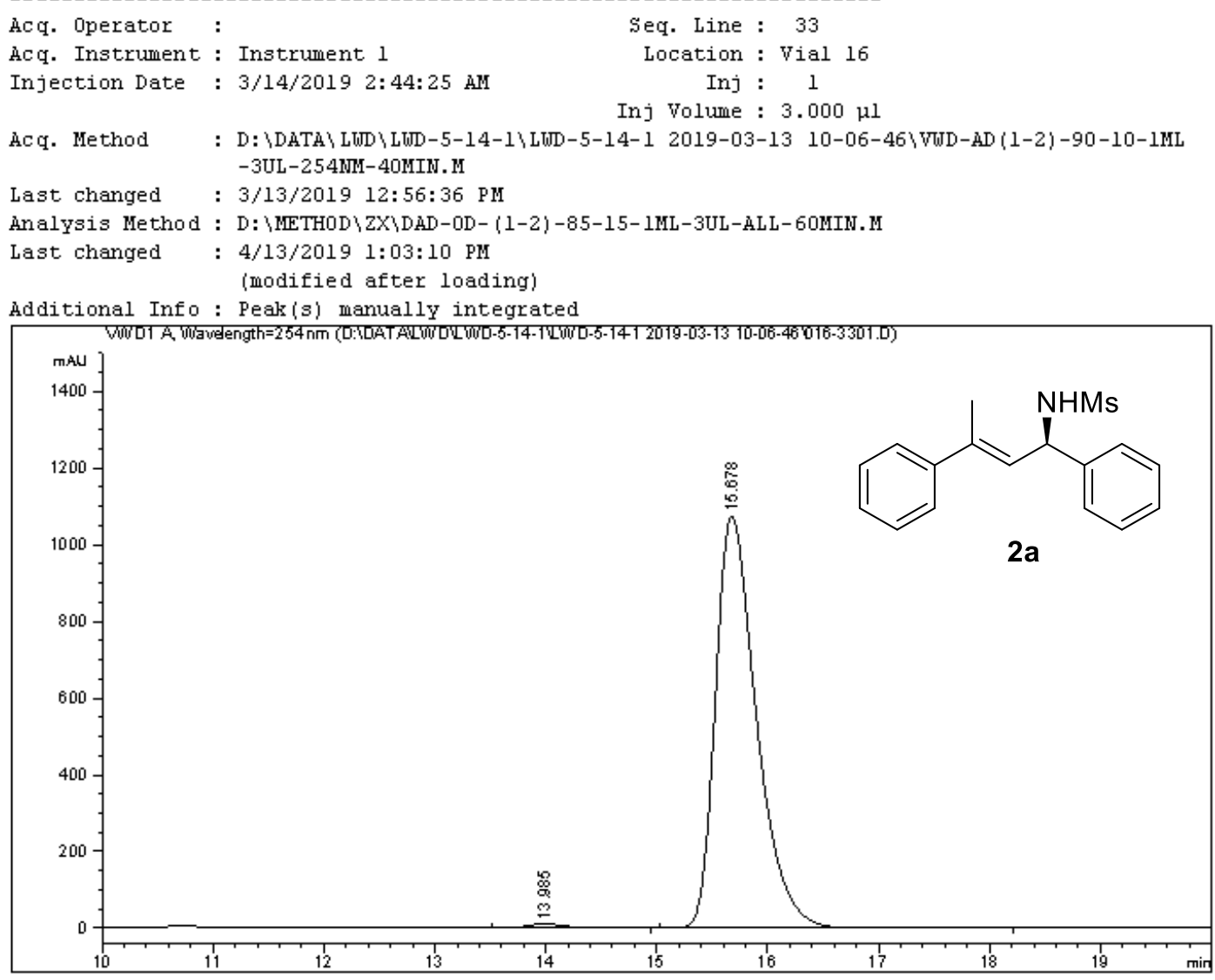

Area Percent Report

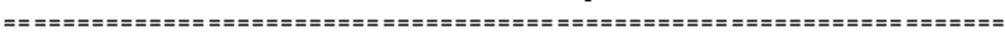

$\begin{array}{lll}\text { Sorted BY } & : & \text { Signal } \\ \text { Multiplier } & : & 1.0000 \\ \text { Dilution } & : & 1.0000\end{array}$

Use Multiplier \& Dilution Factor with ISTDs

Signal 1: VWDl A, Wavelength=254 nm

\begin{tabular}{|c|c|c|c|c|c|c|}
\hline $\begin{array}{c}\mathrm{Peak} \\
\#\end{array}$ & $\begin{array}{c}\text { RetTime } \\
\text { [min] }\end{array}$ & Type & $\begin{array}{l}\text { Width } \\
\text { [min] }\end{array}$ & $\begin{array}{c}\text { Area } \\
{\left[\mathrm{m} A U^{*} S\right]}\end{array}$ & $\begin{array}{l}\text { Height } \\
\text { [mAU] }\end{array}$ & $\begin{array}{c}\text { Area } \\
\stackrel{8}{*}\end{array}$ \\
\hline-- & & & -- & ------ & --------- & ------- \\
\hline 1 & 13.985 & $\mathrm{BB}$ & 0.3590 & 238.01146 & 9.88260 & 0.8578 \\
\hline 2 & 15.678 & $\mathrm{BB}$ & 0.3908 & $2.75093 \mathrm{e} 4$ & 1074.27747 & 99.1422 \\
\hline Tot & 8: & & & $2.77473 \mathrm{e} 4$ & 1084.16006 & \\
\hline
\end{tabular}


Data File D: \DATA $\backslash$ LS \LSL-4-13-1\LSL-4-13-1 2019-03-14 10-11-16\011-0501.D Sample Name: ZX-3-81-N(Me) 2-RAC

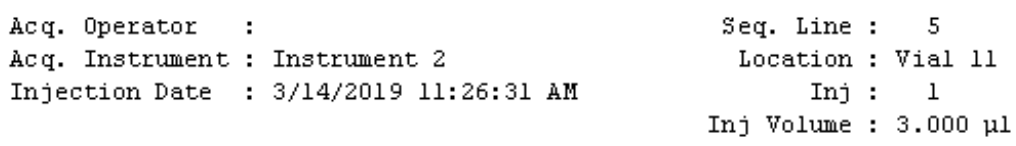

Acq. Method : D: \DATA $\backslash$ LSL $\backslash$ LSL-4-13-1 \LL-4-13-1 2019-03-14 10-11-16 DAD-0D (1-2) -95-5-1ML3UL-ALL-50MIN.M

Last changed : 6/23/2018 3:17:51 PM

Analysis Method : D: \METHOD LGY VWD-AD (1-2)-80-20-1ML-5UL-210NM-40MIN.M

Last changed : 6/14/2019 2:55:59 PM

Additional Info : Peak (s) manually integrated

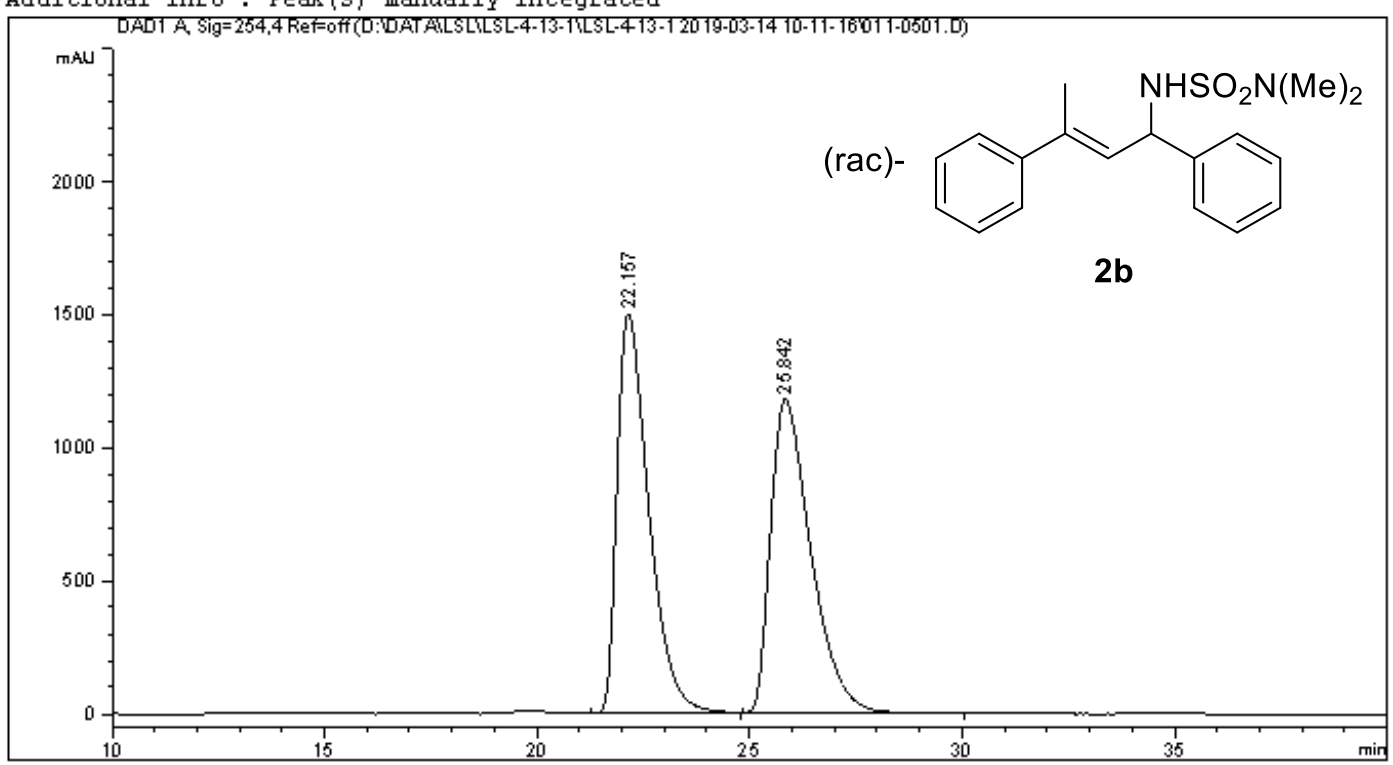

\begin{tabular}{|c|c|c|}
\hline Sorted By & : & Signal \\
\hline Multiplier & : & 1.0000 \\
\hline Dilution & : & 1.0000 \\
\hline
\end{tabular}

Use Multiplier \& Dilution Factor with ISTDs

Signal 1: DADl À, Sig=254, 4 Ref=off

\begin{tabular}{|c|c|c|c|c|c|c|}
\hline $\begin{array}{c}\text { Peak } \\
\#\end{array}$ & $\begin{array}{c}\text { RetTime } \\
\text { [min] }\end{array}$ & Type & $\begin{array}{l}\text { Width } \\
\text { [min] }\end{array}$ & $\begin{array}{c}\text { Area } \\
{\left[\mathrm{m} \mathrm{AU}^{+} \mathrm{s}\right]}\end{array}$ & $\begin{array}{l}\text { Height } \\
\text { [mÄ] }\end{array}$ & $\begin{array}{c}\text { Area } \\
\stackrel{5}{8}\end{array}$ \\
\hline--1 & - & & 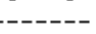 & ---------- & ---------- & "----- \\
\hline 1 & 22.157 & $\mathrm{BB}$ & 0.7717 & $7.78629 \mathrm{e} 4$ & 1499.84729 & 50.1134 \\
\hline 2 & 25.842 & $\mathrm{BB}$ & 0.9949 & $7.75106 \mathrm{e} 4$ & 1179.22327 & 49.8866 \\
\hline \multicolumn{4}{|c|}{ Totals : } & $1.55373 \mathrm{e} 5$ & 2679.07056 & \\
\hline
\end{tabular}


Data File D: \DATA $\backslash$ LS \LSL-4-13-1\LSL-4-13-1 2019-03-14 10-11-16\012-0601.D Sample Name: $2 X-3-83-N($ Me $) 2$

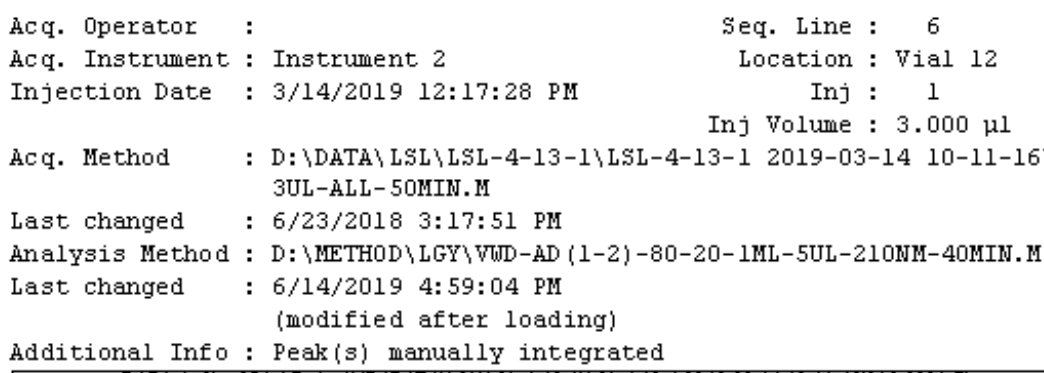
3UL-ALL-5OMIN. M

Last changed : 6/23/2018 3:17:51 PM

Analysis Method : D: \METHOD LGY VWD-AD (1-2)-80-20-1ML-5UL-210NM-40MIN. M

Last changed : 6/14/2019 4:59:04 PM

(modified after loading)

Additional Info : Peak (s) manually integrated

Ac q. Method : D: \DATA $\backslash$ LSL \LSL-4-13-1 \LSL-4-13-1 2019-03-14 10-11-16 DAD-0D (1-2)-95-5-1ML-

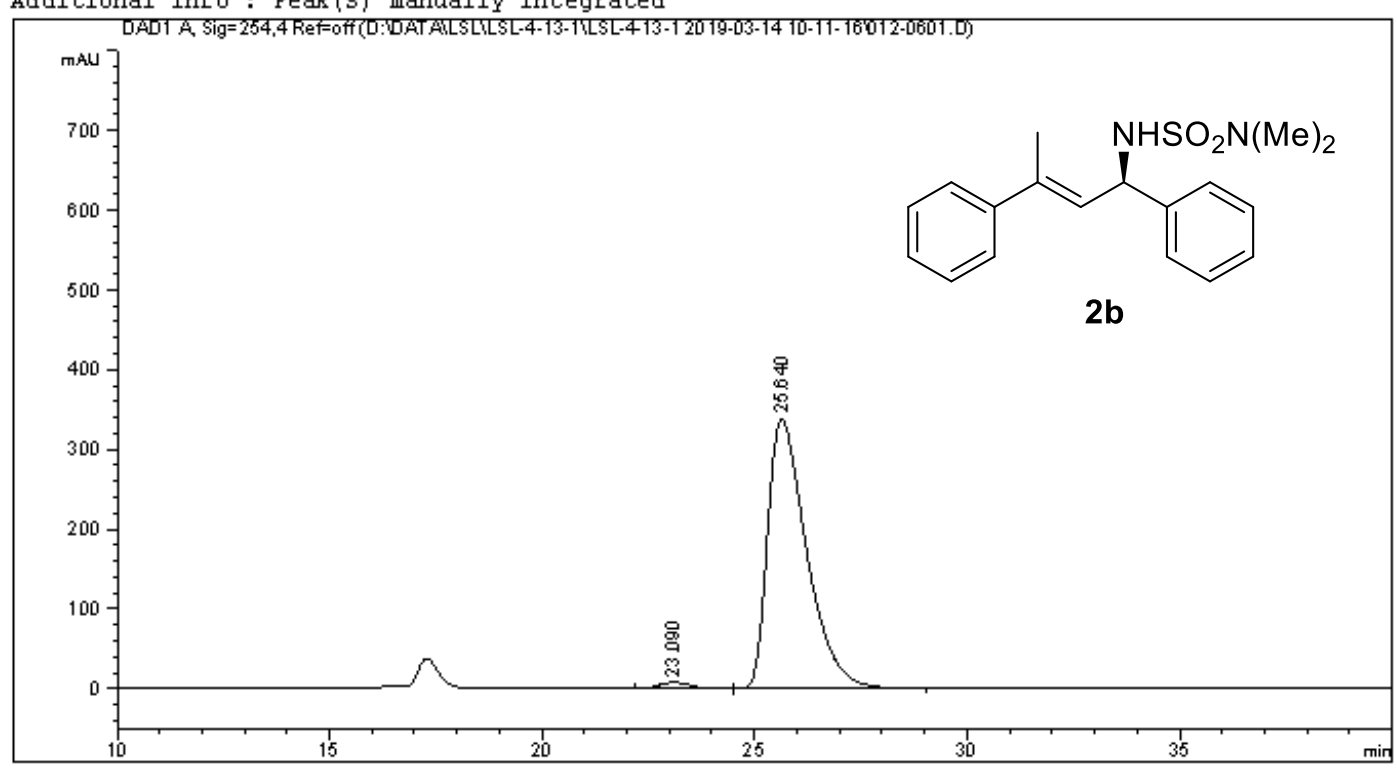

Area Percent Report

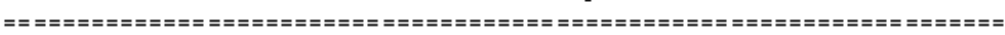

$\begin{array}{lcc}\text { Sorted By } & : & \text { Signal } \\ \text { Multiplier } & : & 1.0000 \\ \text { Dilution } & : & 1.0000 \\ \text { Use Multiplier } & \text { \& Dilution } & \text { Factor with } \\ \text { ISTDs }\end{array}$

Signal 1: DADl A, Sig=254, 4 Ref=off

\begin{tabular}{|c|c|c|c|c|c|c|}
\hline $\begin{array}{c}\text { Peak } \\
\#\end{array}$ & $\begin{array}{c}\text { RetTime } \\
\text { [min] }\end{array}$ & Type & $\begin{array}{l}\text { Width } \\
\text { [min] }\end{array}$ & $\begin{array}{c}\text { Area } \\
\text { [mAUt's] }\end{array}$ & $\begin{array}{l}\text { Height } \\
\text { [mäU] }\end{array}$ & $\begin{array}{c}\text { Area } \\
\stackrel{8}{*}\end{array}$ \\
\hline---1 & $\mid------$ & & ------ & ------ & ------- & ----- \\
\hline 1 & 23.090 & $\mathrm{BB}$ & 0.5882 & 388.26770 & 7.78101 & 1.7781 \\
\hline 2 & 25.640 & $\mathrm{BB}$ & 0.9672 & $2.14477 \mathrm{e} 4$ & 337.59161 & 98.2219 \\
\hline $\mathrm{tal}$ & : & & & $18359 \mathrm{e} 4$ & 345.37263 & \\
\hline
\end{tabular}


Data File D: \DATA \LD $\backslash$ LWD-5-14-1 LWD-5-14-1 2019-03-13 10-06-46\011-2801.D

Sample Name: $2 X-3-81-T s-R A C$

\begin{tabular}{|c|c|c|}
\hline Acq. Operator & : & Seq. Line : 28 \\
\hline Ac q. Instrument & : Instrument 1 & Location : Vial 11 \\
\hline Injection Date & : 3/13/2019 11:20:21 PM & 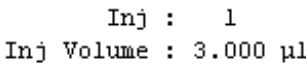 \\
\hline
\end{tabular}

Ac q. Method : D: \DATA \LWD LWD-5-14-1 \LWD-5-14-1 2019-03-13 10-06-46 VWD-AD (1-2) -90-10-1ML $-3 \mathrm{UL}-254 \mathrm{MM}-40 \mathrm{MIN}$. M

Last changed : 3/13/2019 12:56:36 PM

Analysis Method : D: \METHOD XZC \VID-IB (1-6)-98-2-1ML-5UL-210MM-35MIN.M

Last changed : 4/12/2019 7:51:36 PM

(modified after loading)

Additional Info : Peak (s) manually integrated

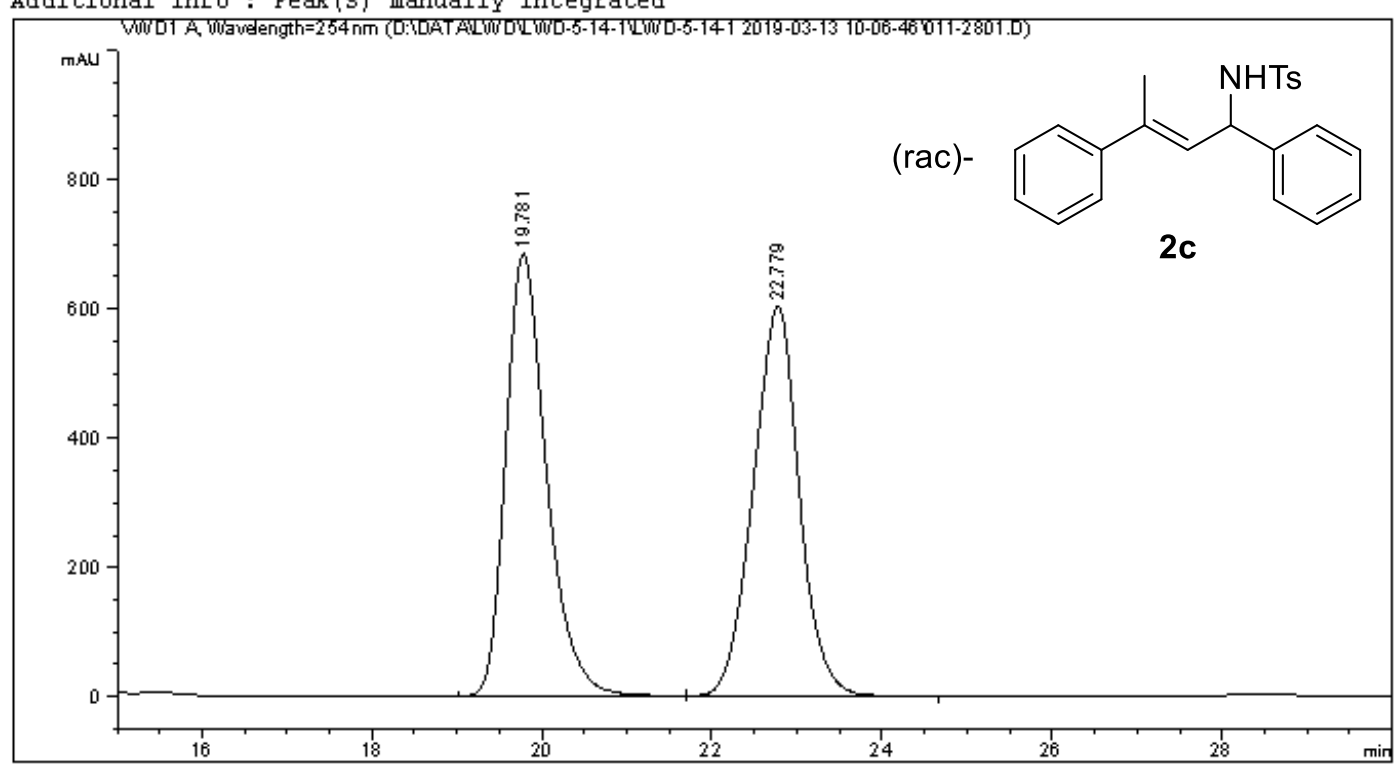

Area Percent Report

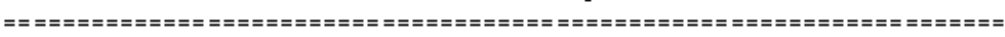

$\begin{array}{lcc}\text { Sorted By } & : & \text { Signal } \\ \text { Multiplier } & : & 1.0000 \\ \text { Dilution } & : & 1.0000 \\ \text { Use Multiplier } & \text { \& Dilution } & \text { Factor with }\end{array}$

Signal 1: VWDl A, Wavelength=254 nm

\begin{tabular}{|c|c|c|c|c|c|c|}
\hline $\begin{array}{c}\text { Peak } \\
\#\end{array}$ & $\begin{array}{c}\text { RetTime } \\
\text { [min] }\end{array}$ & Type & $\begin{array}{l}\text { Width } \\
\text { [min] }\end{array}$ & $\begin{array}{c}\text { Area } \\
\text { [mAUt's] }\end{array}$ & 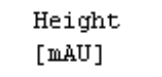 & $\begin{array}{c}\text { Area } \\
\stackrel{8}{*}\end{array}$ \\
\hline--- & |---- & & -------1 & -------- & $--------\mid$ & ----- \\
\hline 1 & 19.781 & BB & 0.5075 & $2.28126 \mathrm{e} 4$ & 685.01813 & 50.1410 \\
\hline 2 & 22.779 & BB & 0.5716 & $2.26843 \mathrm{e} 4$ & 603.43506 & 49.8590 \\
\hline $\mathrm{ta}$ & : & & & 9e4 & 88.45319 & \\
\hline
\end{tabular}


Data File D: \DATA \LD \LWD-5-14-1\LWD-5-14-1 2019-03-13 10-06-46\012-2901.D

Sample Name: $2 X-3-83-T s$

\begin{tabular}{|c|c|c|}
\hline Acq. Operator & : & Seq. Line : 29 \\
\hline Ac q. Instrument & : Instrument 1 & Location : Vial 12 \\
\hline Injection Date & : 3/14/2019 12:01:09 AM & 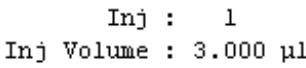 \\
\hline
\end{tabular}

Acq. Method : D: \DATA \LID \LTD-5-14-1 \LWD-5-14-1 2019-03-13 10-06-46 VIDD-AD (1-2)-90-10-1ML $-3 \mathrm{UL}-254 \mathrm{MM}-40 \mathrm{MIN}$. M

Last changed : 3/13/2019 12:56:36 PM

Analysis Method : D: \METHOD XZC \VID-IB (1-6)-98-2-1ML-5UL-210MM-35MIN.M

Last changed : 4/12/2019 7:58:08 PM

(modified after loading)

Additional Info : Peak (s) manually integrated

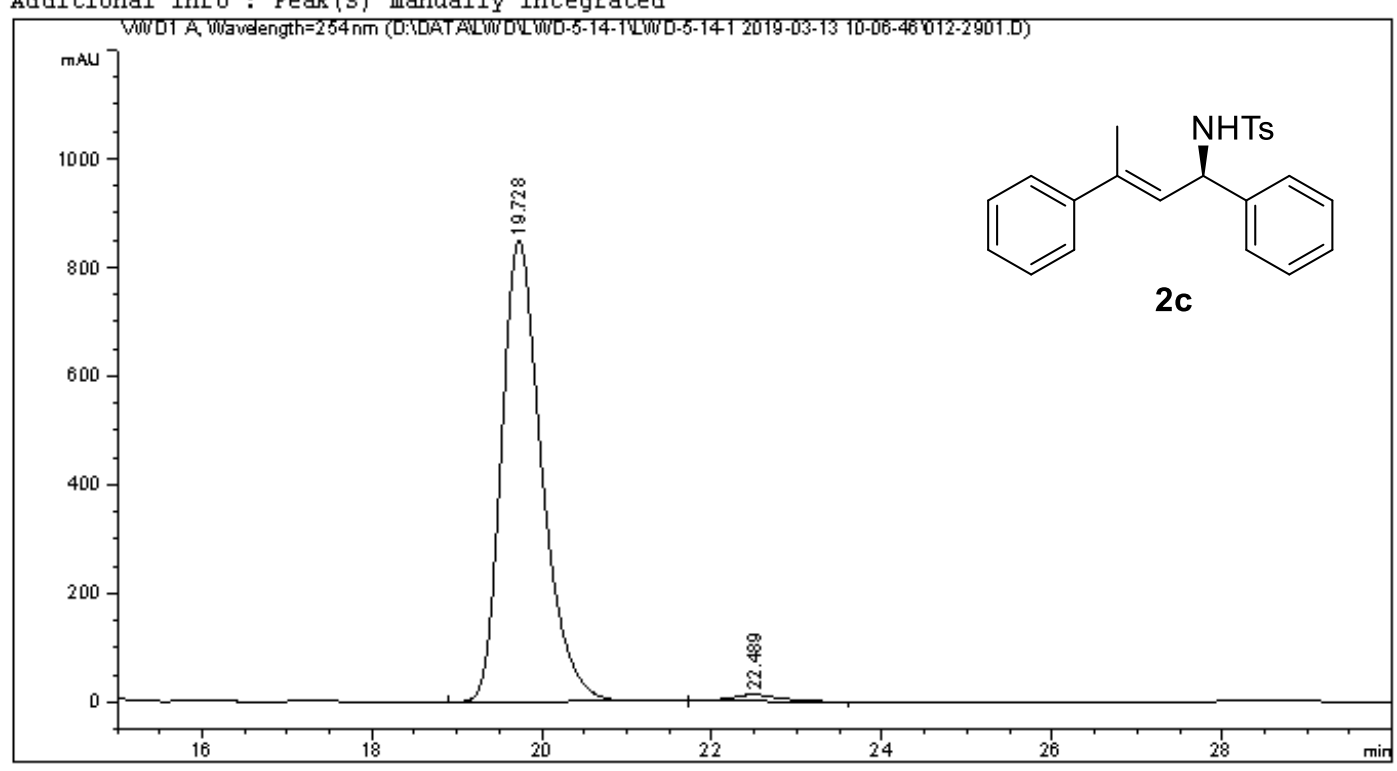

Area Percent Report

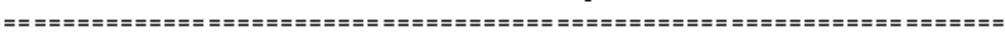

$\begin{array}{lcc}\text { Sorted By } & : & \text { Signal } \\ \text { Multiplier } & : & 1.0000 \\ \text { Dilution } & : & 1.0000 \\ \text { Use Multiplier } & \text { \& Dilution } & \text { Factor with } \\ \end{array}$

Signal 1: VWDl A, Wavelength=254 nm

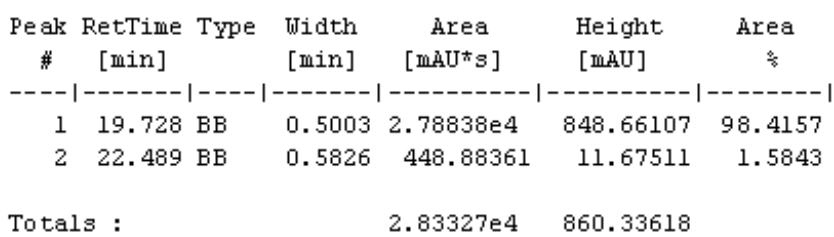


Data File D: \DATA \LYH LYH-4-656-RAC \LYH-4-656-RAC-FURAN 2019-04-12 14-40-53\081-2001.D Sample Name: ZX-3-81-4F-RAC

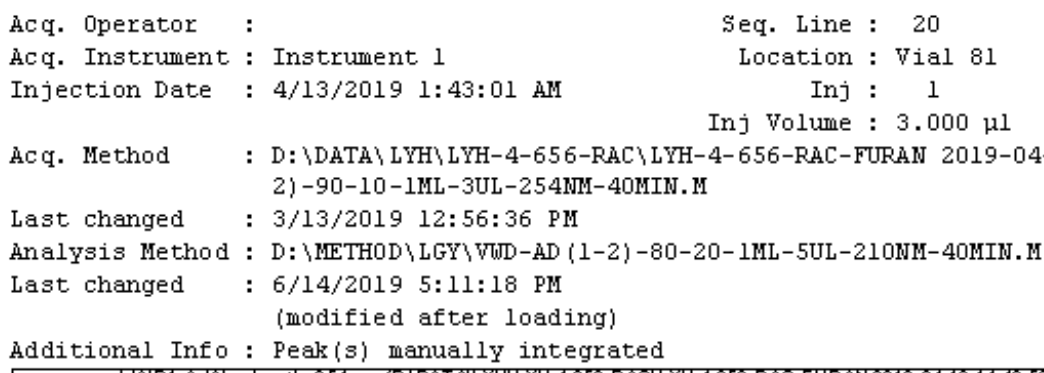
2) $-90-10-1 M L-3 U L-254 M M-40 M I N . M$

Last changed : $3 / 13 / 2019$ 12:56:36 PM

Analysis Method : D: \METHOD $\backslash$ LGY VWD-AD (1-2)-80-20-1ML-5UL-210NM-40MIN. M

Last changed : 6/14/2019 5:11:18 PM

(modified after loading)

Additional Info : Peak (s) manually integrated

Ac q. Method : D: \DATA $\backslash$ LYH $\backslash$ LYH-4-656-RAC LYH-4-656-RAC-FURAN 2019-04-12 14-40-53 VTD-AD (1-

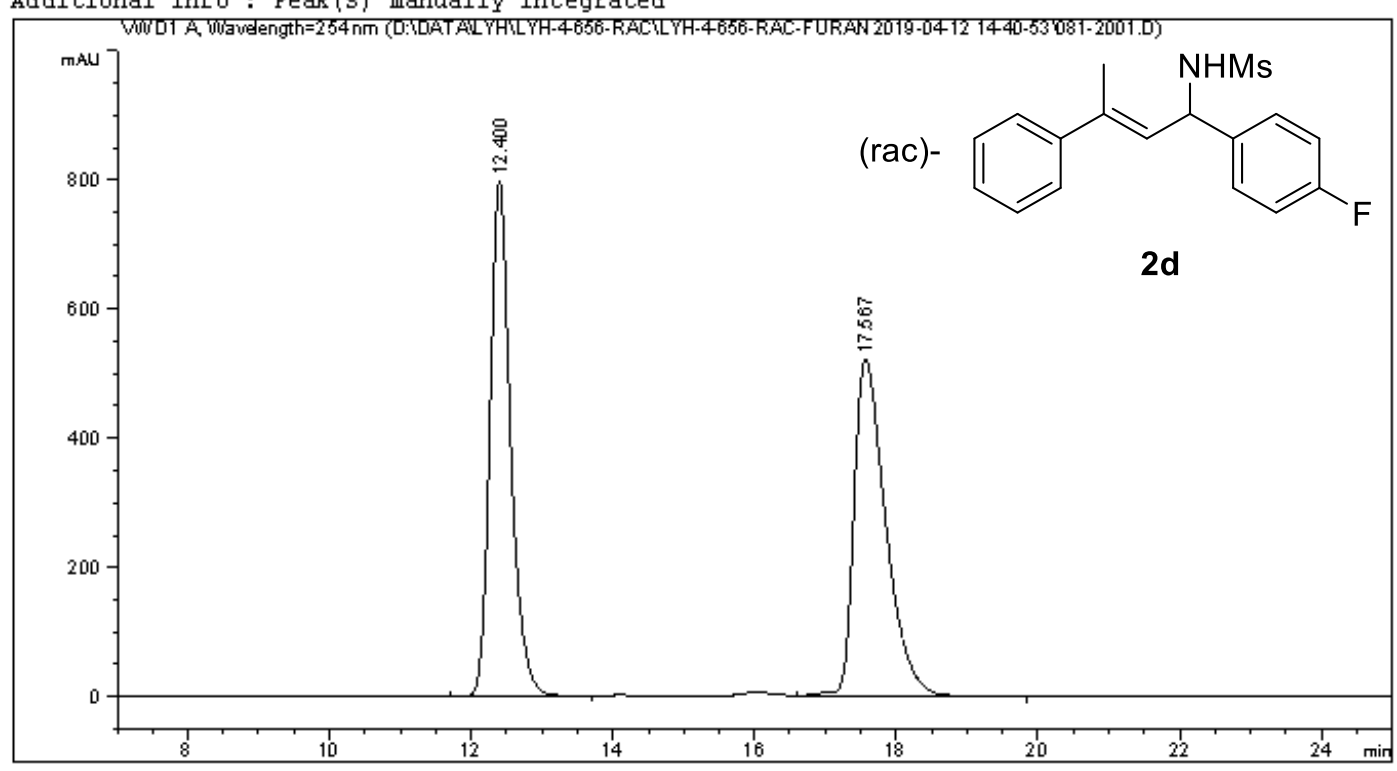

Area Percent Report

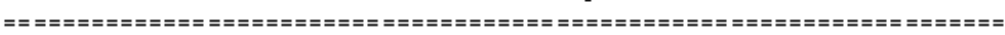

$\begin{array}{lcc}\text { Sorted By } & : & \text { Signal } \\ \text { Multiplier } & : & 1.0000 \\ \text { Dilution } & : & 1.0000 \\ \text { Use Multiplier } & \& \text { Dilution } & \text { Factor with }\end{array}$

Signal 1: VWDl A, Wavelength=254 nm

\begin{tabular}{|c|c|c|c|c|c|c|}
\hline $\begin{array}{c}\text { Peak } \\
\#\end{array}$ & $\begin{array}{c}\text { RetTime } \\
\text { [min] }\end{array}$ & Type & $\begin{array}{l}\text { Width } \\
\text { [min] }\end{array}$ & $\begin{array}{c}\text { Area } \\
\text { [mAUt's] }\end{array}$ & $\begin{array}{l}\text { Height } \\
\text { [mAU] }\end{array}$ & $\begin{array}{c}\text { Area } \\
\stackrel{8}{8}\end{array}$ \\
\hline-- & & & & -------- & -------- & ------ \\
\hline 1 & 12.400 & BB & 0.3038 & $1.60673 \mathrm{e} 4$ & 798.43665 & 49.7012 \\
\hline 2 & 17.567 & VB & 0.4747 & $1.62605 \mathrm{e} 4$ & 521.54669 & 50.2988 \\
\hline $\mathrm{tal}$ & : & & & $23278 \mathrm{e} 4$ & 319.98334 & \\
\hline
\end{tabular}


Data File D: \DATA \LYH \YYH-4-656-RAC \LY-4-656-RAC-FURAN 2019-04-12 14-40-53\082-2101.D Sample Name: $2 X-3-81-4 F$

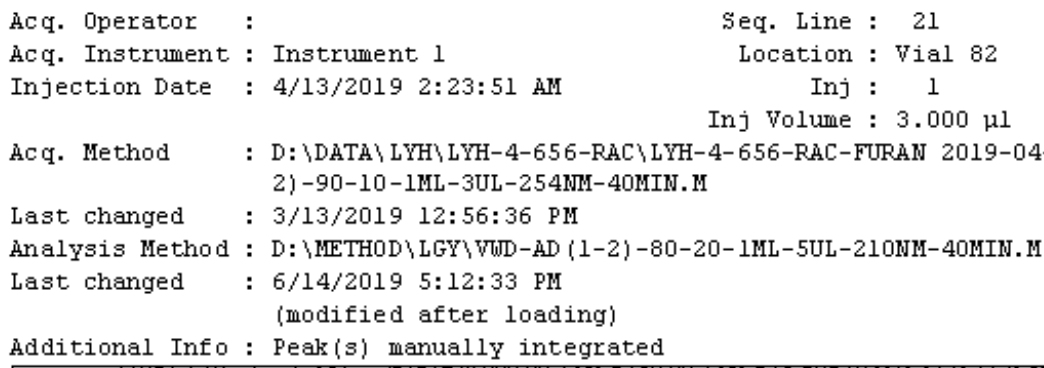
2) $-90-10-1 M L-3 U L-254 M M-40$ MIN. M

Last changed : $3 / 13 / 2019$ 12:56:36 PM

Analysis Method : D: \METHOD $\backslash$ LGY $\backslash$ VDD-AD (1-2)-80-20-1ML-5UL-210NM-40MIN. M

Last changed : 6/14/2019 5:12:33 PM

(modified after loading)

Additional Info : Peak (s) manually integrated

Ac q. Method : D: \DATA $\backslash$ LYH $\backslash$ LYH-4-656-RAC $\backslash$ LYH-4-656-RAC-FURAN 2019-04-12 14-40-53 VTD-AD (1-

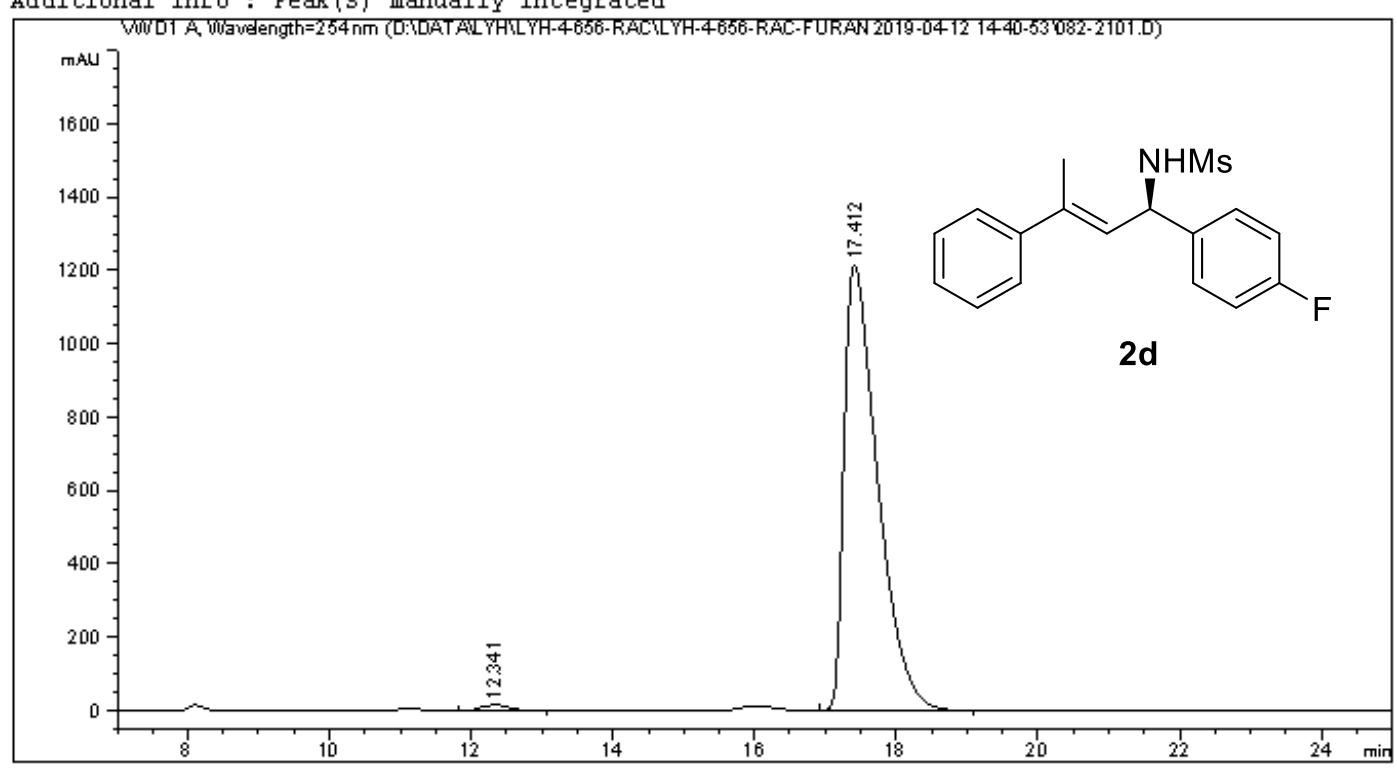

Area Percent Report

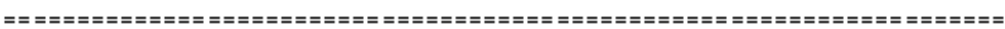

$\begin{array}{lcc}\text { Sorted By } & : & \text { Signal } \\ \text { Multiplier } & : & 1.0000 \\ \text { Dilution } & : & 1.0000 \\ \text { Use Multiplier } & \text { \& Dilution } & \text { Factor with }\end{array}$

Signal 1: VTD 1 , Wavelength=254 nm

\begin{tabular}{|c|c|c|c|c|c|}
\hline $\begin{array}{c}\text { Peak } \\
\#\end{array}$ & $\begin{array}{l}\text { RetTime Type } \\
\text { [min] }\end{array}$ & $\begin{array}{l}\text { Width } \\
\text { [min] }\end{array}$ & $\begin{array}{c}\text { Area } \\
\text { [mAUt's] }\end{array}$ & 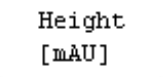 & $\begin{array}{c}\text { Area } \\
\stackrel{8}{*}\end{array}$ \\
\hline-- & -------1 & -------1 & --------- & $|---------|$ & ----- \\
\hline 1 & $12.341 \mathrm{BB}$ & 0.4048 & 382.90158 & 15.25454 & 0.9480 \\
\hline 2 & $17.412 \mathrm{BB}$ & 0.4973 & 4. $00064 \mathrm{e} 4$ & 1214.49817 & 99.0520 \\
\hline ota & : & & $03894 \mathrm{e} 4$ & 229.75271 & \\
\hline
\end{tabular}


Data File D: \DATA \LY \LYH-4-656-RAC \LYH-4-656-RAC-FURAN 2019-04-12 14-40-53\083-2201.D Sample Name: $2 X-3-81-4 C l-R A C$

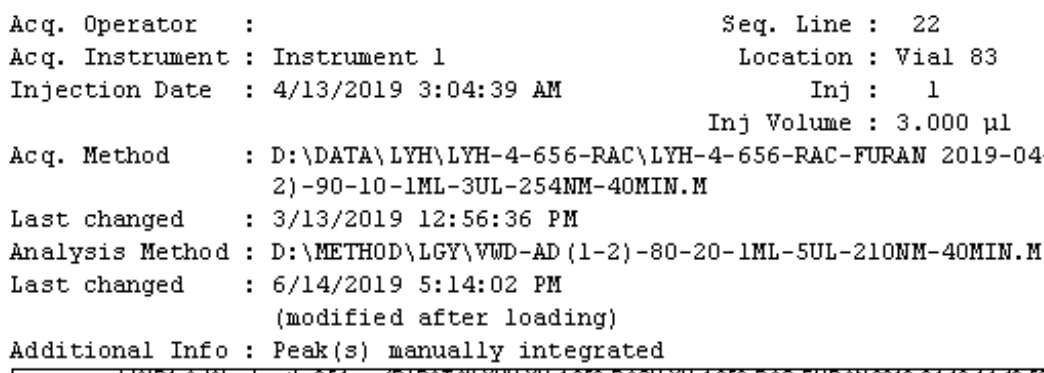
2) $-90-10-1 M L-3 U L-254 M M-40 M I N . M$

Last changed : $3 / 13 / 2019$ 12:56:36 PM

Analysis Method : D: \METHOD $\backslash$ LGY $\backslash$ VDD-AD (1-2)-80-20-1ML-5UL-210NM-40MIN. M

Last changed : 6/14/2019 5:14:02 PM

(modified after loading)

Additional Info : Peak (s) manually integrated

Ac q. Method : D: \DATA $\backslash$ LYH $\backslash$ LYH-4-656-RAC $\backslash$ LYH-4-656-RAC-FURAN 2019-04-12 14-40-53 VTD-AD (1-

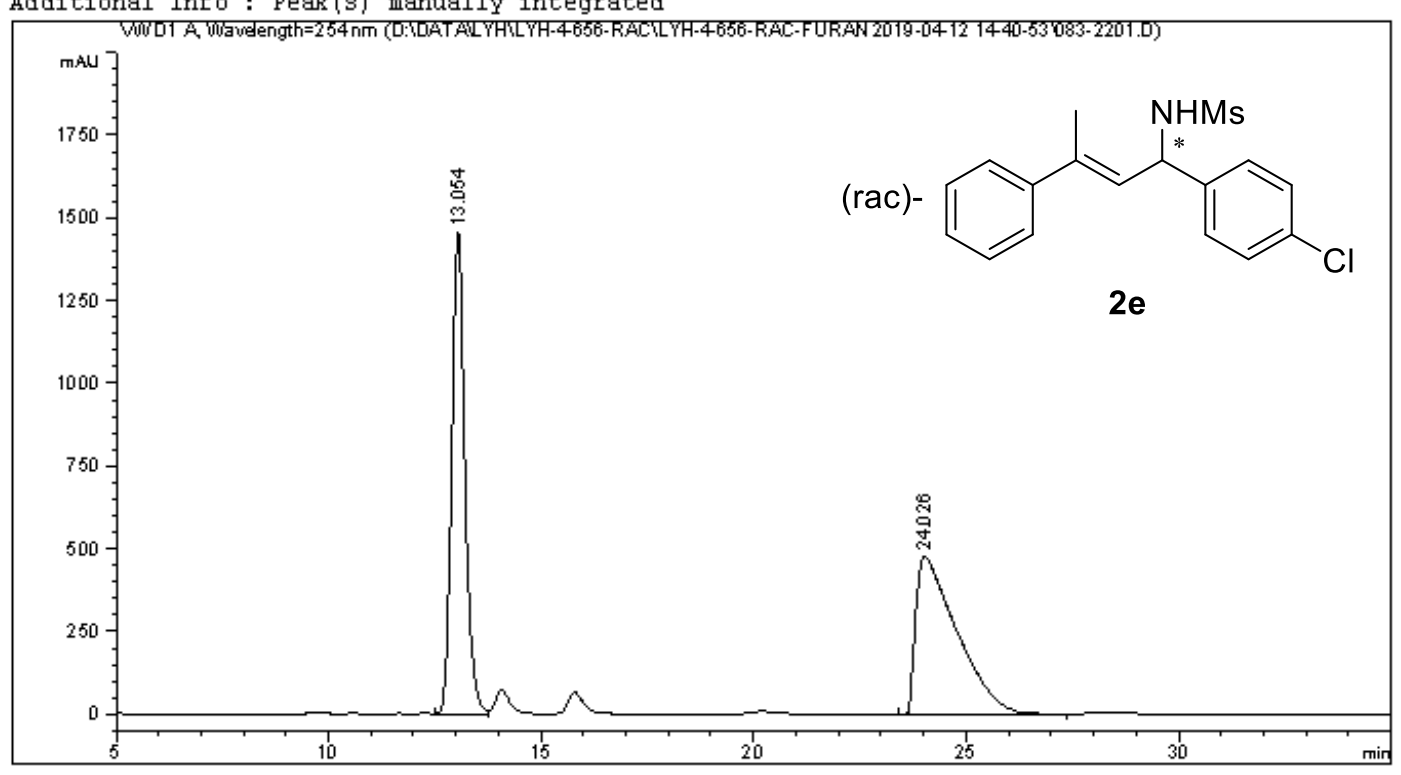

Area Percent Report

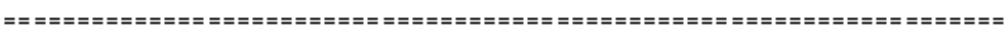

$\begin{array}{lcc}\text { Sorted By } & : & \text { Signal } \\ \text { Multiplier } & : & 1.0000 \\ \text { Dilution } & : & 1.0000 \\ \text { Use Multiplier } & \text { \& Dilution } & \text { Factor with }\end{array}$

Signal 1: VWDl A, Wavelength=254 nm

\begin{tabular}{|c|c|c|c|c|c|}
\hline $\begin{array}{c}\text { Peak } \\
\#\end{array}$ & $\begin{array}{l}\text { RetTime Type } \\
\text { [min] }\end{array}$ & $\begin{array}{l}\text { Width } \\
\text { [min] }\end{array}$ & $\begin{array}{c}\text { Area } \\
\text { [mÁnt*s] }\end{array}$ & $\begin{array}{l}\text { Height } \\
\text { [mAU] }\end{array}$ & $\begin{array}{c}\text { Area } \\
\stackrel{\circ}{*}\end{array}$ \\
\hline---1 & |--- & -------1 & -------- & |--------- & $--0---4$ \\
\hline 1 & $13.054 \mathrm{VV}$ & 0.3257 & $3.14686 \mathrm{e} 4$ & 1458.58313 & 49.6630 \\
\hline 2 & $24.026 \mathrm{BB}$ & 0.9498 & $3.18957 \mathrm{e} 4$ & 475.95847 & 50.3370 \\
\hline \multicolumn{3}{|c|}{ Totals : } & $6.33643 \mathrm{e} 4$ & 1934.54160 & \\
\hline
\end{tabular}


Data File D: \DATA \LYH \LYH-4-656-RAC \LY-4-656-RAC-FURAN 2019-04-12 14-40-53\084-2301.D Sample Name: $2 X-3-81-4 C 1$

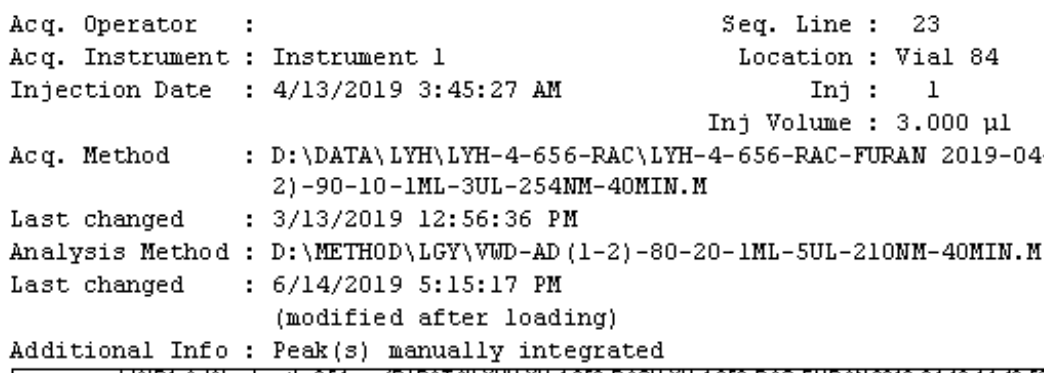
2) $-90-10-1 M L-3 U L-254 M M-40 M I N . M$

Last changed : $3 / 13 / 2019$ 12:56:36 PM

Analysis Method : D: \METHOD $\backslash$ LGY VWD-AD (1-2)-80-20-1ML-5UL-210NM-40MIN. M

Last changed : 6/14/2019 5:15:17 PM

(modified after loading)

Additional Info : Peak (s) manually integrated

Ac q. Method : D: \DATA $\backslash$ LYH $\backslash$ LYH-4-656-RAC LYH-4-656-RAC-FURAN 2019-04-12 14-40-53 VTD-AD (1-

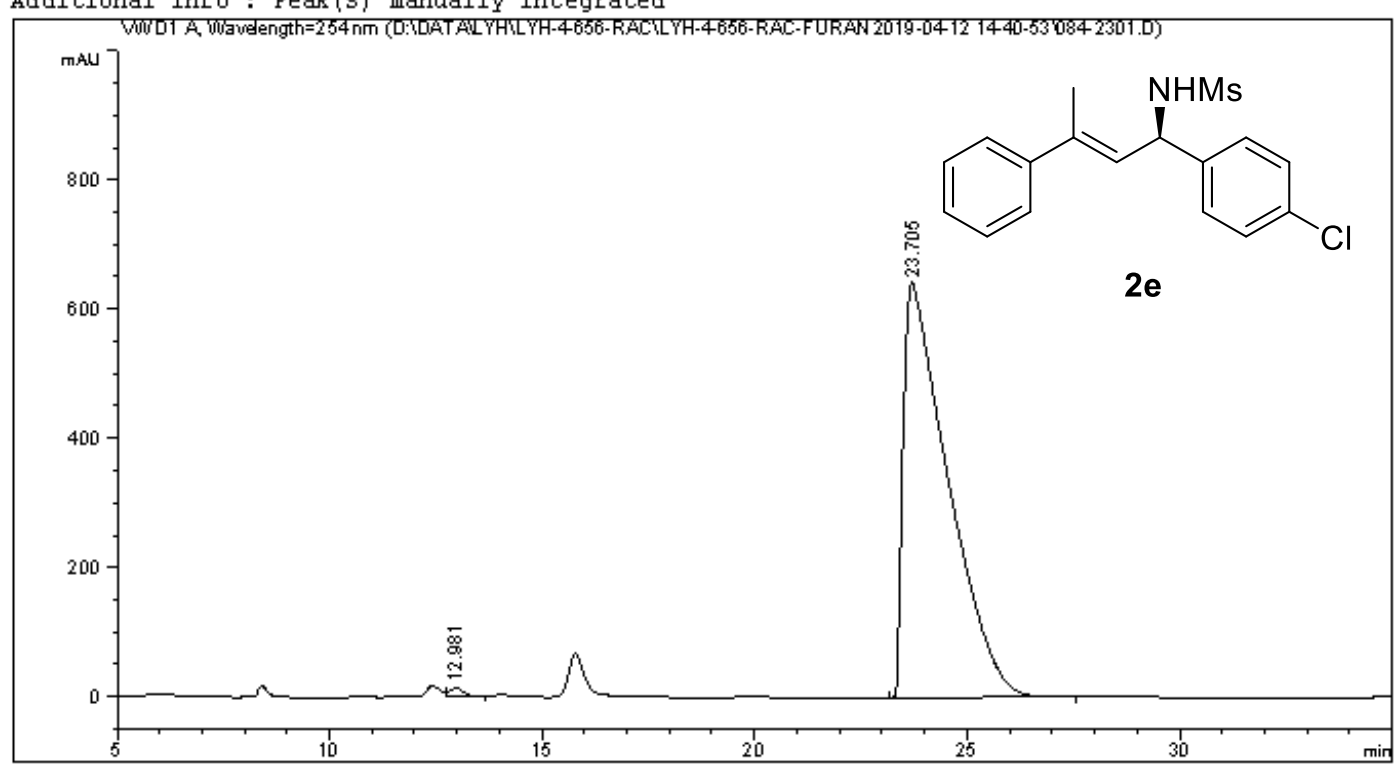

Area Percent Report

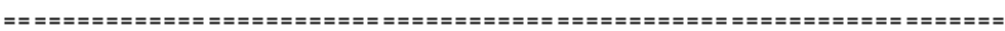

$\begin{array}{lcc}\text { Sorted By } & : & \text { Signal } \\ \text { Multiplier } & : & 1.0000 \\ \text { Dilution } & : & 1.0000 \\ \text { Use Multiplier } & \text { \& Dilution } & \text { Factor with }\end{array}$

Signal 1: VTD 1 , Wavelength=254 nm

\begin{tabular}{|c|c|c|c|c|c|}
\hline $\begin{array}{c}\mathrm{eak} \\
\#\end{array}$ & $\begin{array}{l}\text { RetTime Type } \\
\text { [min] }\end{array}$ & $\begin{array}{l}\text { Width } \\
\text { [min] }\end{array}$ & $\begin{array}{c}\text { Area } \\
\text { [minUt's] }\end{array}$ & $\begin{array}{l}\text { Height } \\
\text { [miU] }\end{array}$ & $\begin{array}{c}\text { Àrea } \\
\stackrel{8}{*}\end{array}$ \\
\hline & & & 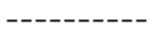 & & 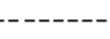 \\
\hline 1 & & & 342. & 15. & 0.7234 \\
\hline 2 & & 823 & $4.69483 \mathrm{e} 4$ & 641.92816 & 99.2766 \\
\hline
\end{tabular}

Totals : $\quad 4.72904 \mathrm{e} 4 \quad 657.30815$ 
Data File D: \DATA \LD\LWD-5-14-1 LWD-5-14-1 2019-03-13 10-06-46\017-3401.D

Sample Name: $2 X-3-81-40 \mathrm{Me}-\mathrm{RAC}$

\begin{tabular}{|c|c|c|}
\hline Acq. Operator & : & seq. Line : 34 \\
\hline Ac q. Instrument & : Instrument 1 & Location : Vial 17 \\
\hline Injection Date & : $3 / 14 / 20193: 25: 12$ AM & $\begin{array}{rlc}\text { Inj } & : & 1 \\
\text { Inj Volume } & : & 3.000 \mu \mathrm{l}\end{array}$ \\
\hline
\end{tabular}

Acq. Method : D: \DATA \LID \LTD-5-14-1 \LWD-5-14-1 2019-03-13 10-06-46 VIDD-AD (1-2)-90-10-1ML $-3 \mathrm{UL}-254 \mathrm{NM}-40 \mathrm{MIN}$. M

Last changed : 3/13/2019 12:56:36 PM

Analysis Method : D: \METHOD LGY VWD-AD (1-2)-80-20-1ML-5UL-210NM-40MIN. M

Last changed : 6/14/2019 5:02:44 PM

(modified after loading)

Additional Info : Peak (s) manually integrated

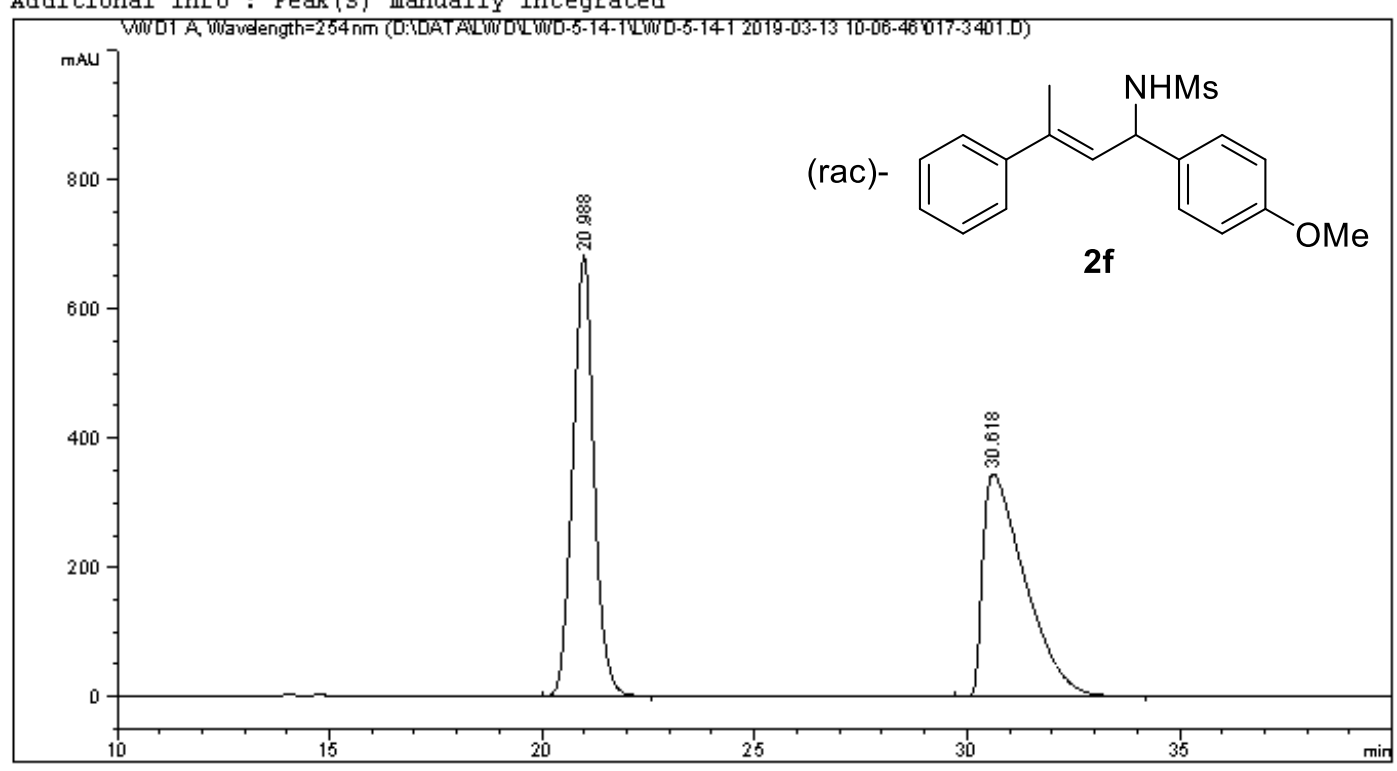

Area Percent Report

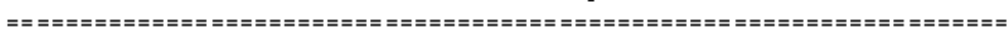

$\begin{array}{lcc}\text { Sorted By } & : & \text { Signal } \\ \text { Multiplier } & : & 1.0000 \\ \text { Dilution } & : & 1.0000 \\ \text { Use Multiplier } & \text { \& Dilution } & \text { Factor with }\end{array}$

Signal 1: VWDl A, Wavelength=254 nm

\begin{tabular}{|c|c|c|c|c|c|c|}
\hline $\begin{array}{c}\mathrm{eak} \\
\#\end{array}$ & $\begin{array}{c}\text { RetTime } \\
\text { [min] }\end{array}$ & Type & $\begin{array}{l}\text { Width } \\
\text { [min] }\end{array}$ & $\begin{array}{c}\text { Area } \\
\text { [mänts }]\end{array}$ & $\begin{array}{l}\text { Height } \\
\text { [m̉U] }\end{array}$ & $\begin{array}{c}\text { Area } \\
\stackrel{8}{*}\end{array}$ \\
\hline & & & & -- & & \\
\hline 1 & & & & 2.357 & 682 & 657 \\
\hline 2 & 30 & $B B$ & 1.0315 & $2.37060 \mathrm{e} 4$ & 344.62805 & 50.1343 \\
\hline
\end{tabular}

Totals : $\quad 4.72850 e 4 \quad 1026.74261$ 
Data File D: \DATA $\backslash L D \backslash L W D-5-14-1 \backslash L$ LD-5-14- 1 2019-03-13 10-06-46\018-3501.D Sample Name: $2 X-3-83-40 M e$

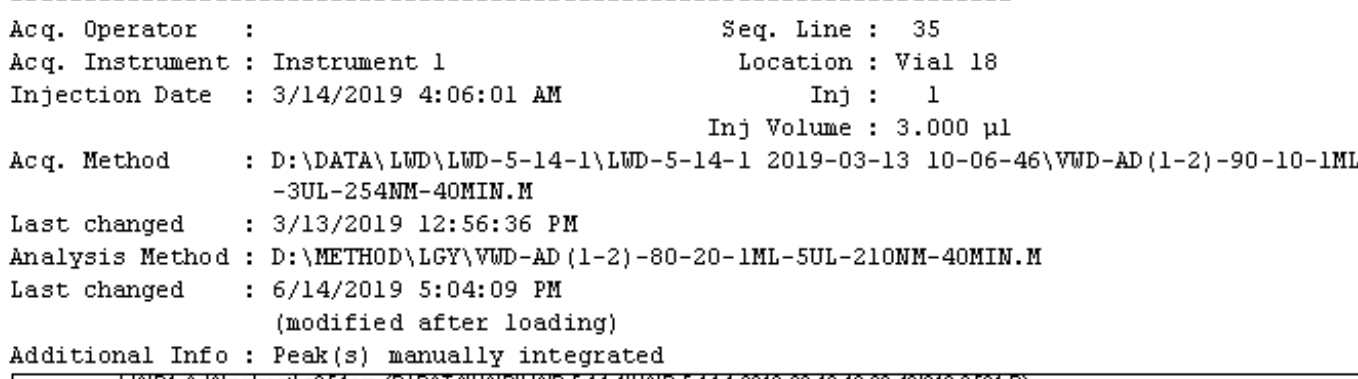

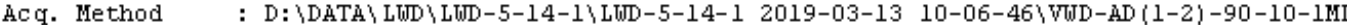
$-3 \mathrm{UL}-254 \mathrm{MM}-40 \mathrm{MIN} . \mathrm{M}$

Last changed : $3 / 13 / 2019$ 12:56:36 PM

Analysis Method : D: \METHOD LGY VWD-AD (1-2)-80-20-1ML-5UL-210NM-40MIN. M

Last changed : 6/14/2019 5:04:09 PM

(modified after loading)

Additional Info : Peak (s) manually integrated

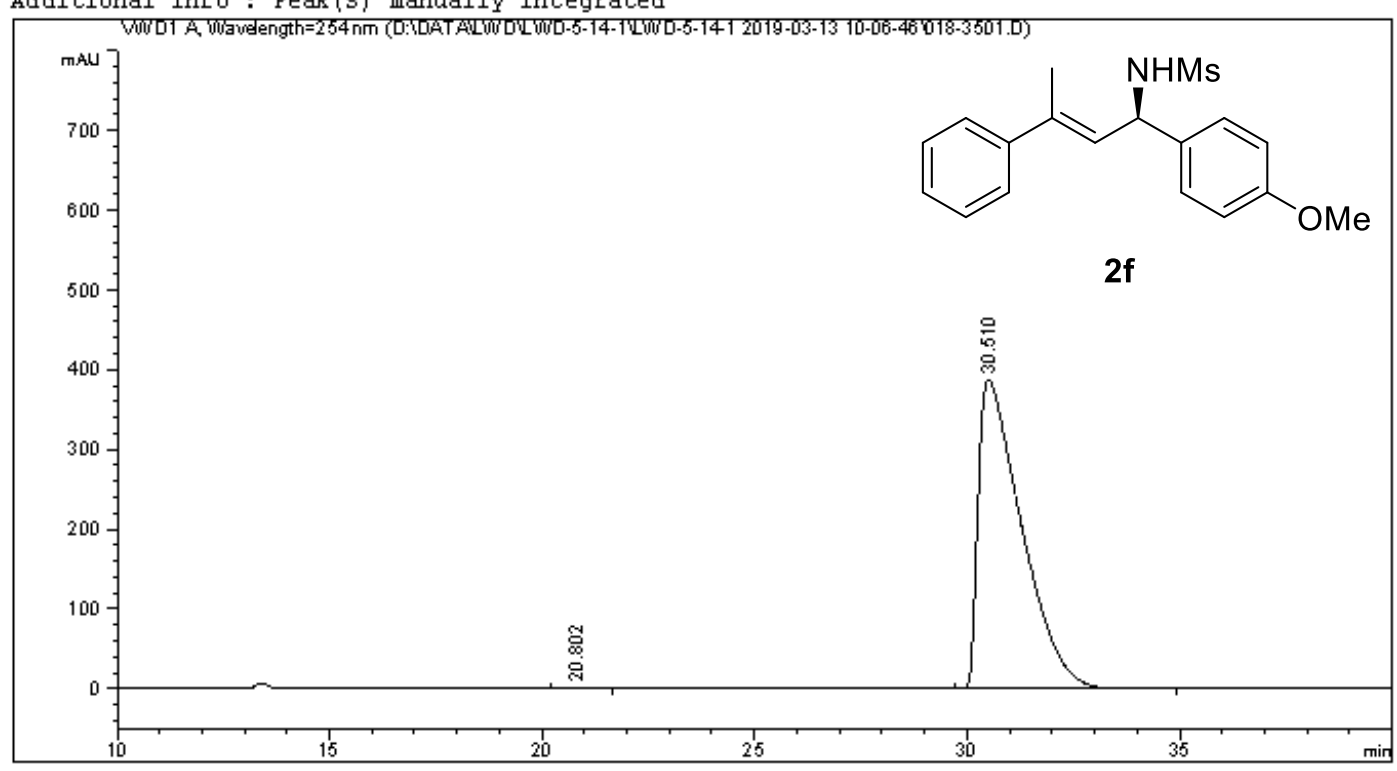

Area Percent Report

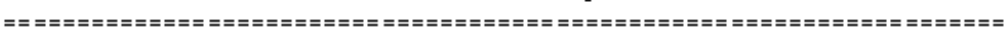

$\begin{array}{lcc}\text { Sorted By } & : & \text { Signal } \\ \text { Multiplier } & : & 1.0000 \\ \text { Dilution } & : & 1.0000 \\ \text { Use Multiplier } & \text { \& Dilution Factor with ISTDs }\end{array}$

Signal 1: VWDl A, Wavelength=254 nm

\begin{tabular}{|c|c|c|c|c|c|}
\hline $\begin{array}{c}\mathrm{Peak} \\
\#\end{array}$ & $\begin{array}{l}\text { RetTime Type } \\
\text { [min] }\end{array}$ & $\begin{array}{l}\text { Width } \\
\text { [min] }\end{array}$ & $\begin{array}{c}\text { Area } \\
{\left[\mathrm{m} \mathrm{HU}^{*} \mathrm{~s}\right]}\end{array}$ & $\begin{array}{l}\text { Height } \\
\text { [mAU] }\end{array}$ & $\begin{array}{c}\text { Area } \\
\stackrel{8}{8}\end{array}$ \\
\hline--1 & $1-$ & -- & --- & 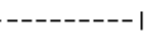 &..- \\
\hline 1 & $20.802 \mathrm{BB}$ & 0.4864 & 82.80405 & 2.51315 & 0.3007 \\
\hline 2 & $30.510 \mathrm{BB}$ & 1.0467 & $2.74582 \mathrm{e} 4$ & 387.51929 & 99.6993 \\
\hline Total & Ls: & & $2.75410 \mathrm{e} 4$ & 390.03243 & \\
\hline
\end{tabular}


Data File D: \DATA \LYH \LYH-4-656-RAC \LY-4-656-RAC-FURAN 2019-04-12 14-40-53\085-2401.D Sample Name: $2 X-3-81-4 M e-R A C$

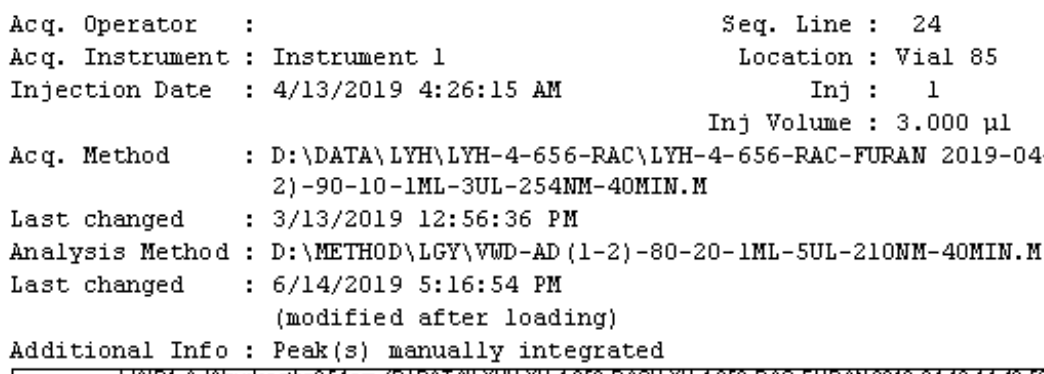
2) $-90-10-1 M L-3 U L-254 M M-40 M I N . M$

Last changed : $3 / 13 / 2019$ 12:56:36 PM

Analysis Method : D: \METHOD LGY VWD-AD (1-2)-80-20-1ML-5UL-210NM-40MIN. M

Last changed : 6/14/2019 5:16:54 PM

(modified after loading)

Additional Info : Peak (s) manually integrated

Ac q. Method : D: \DATA $\backslash$ LYH $\backslash$ LYH-4-656-RAC LYH-4-656-RAC-FURAN 2019-04-12 14-40-53 VTD-AD (1-

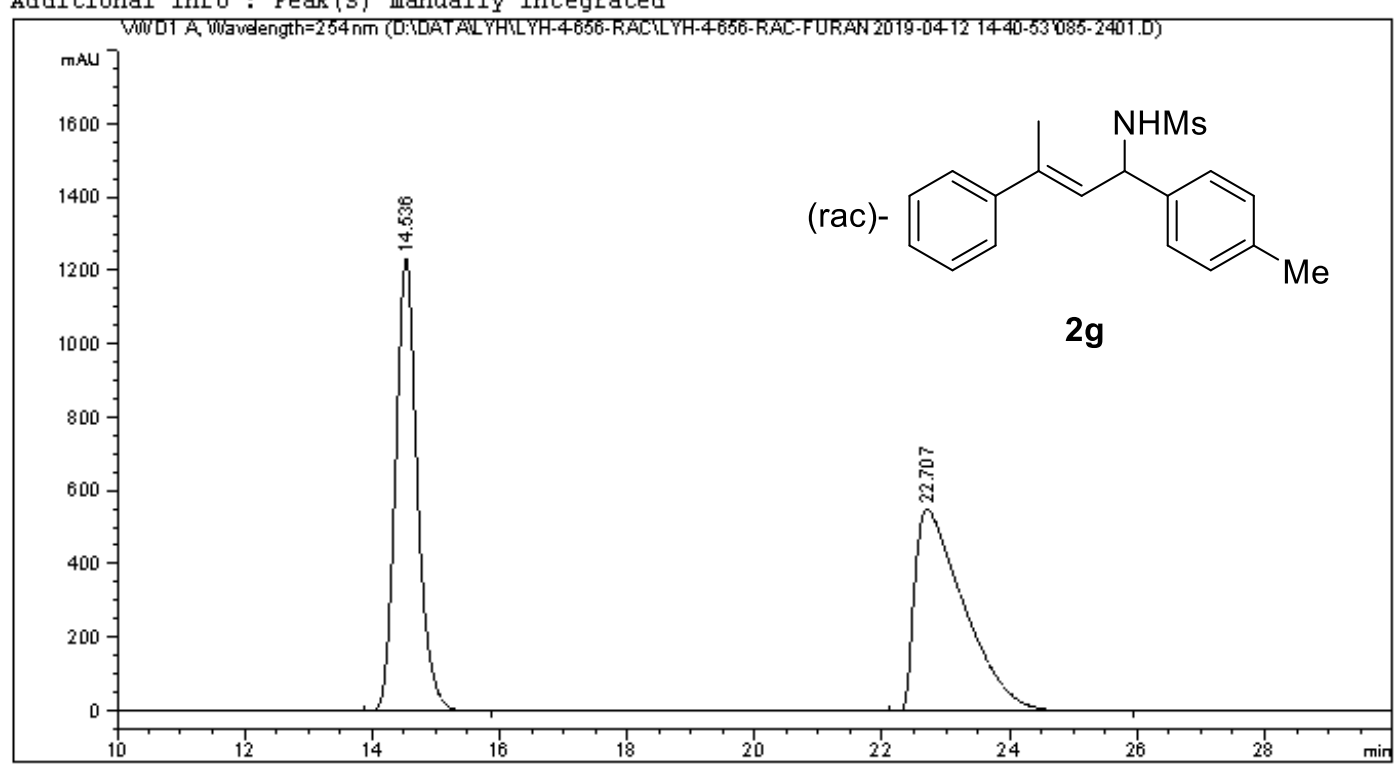

Area Percent Report

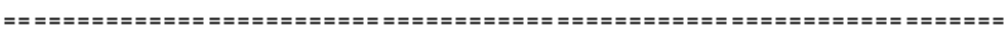

$\begin{array}{lcc}\text { Sorted By } & : & \text { Signal } \\ \text { Multiplier } & : & 1.0000 \\ \text { Dilution } & : & 1.0000 \\ \text { Use Multiplier } & \text { \& Dilution } & \text { Factor with }\end{array}$

Signal 1: VWDl A, Wavelength=254 nm

\begin{tabular}{|c|c|c|c|c|c|}
\hline $\begin{array}{c}\text { Peak } \\
\#\end{array}$ & $\begin{array}{l}\text { RetTime Type } \\
\text { [min] }\end{array}$ & $\begin{array}{l}\text { Width } \\
\text { [min] }\end{array}$ & $\begin{array}{c}\text { Area } \\
\text { [mân*s] }\end{array}$ & $\begin{array}{l}\text { Height } \\
\text { [mAU] }\end{array}$ & $\begin{array}{c}\text { Area } \\
\stackrel{\circ}{*}\end{array}$ \\
\hline---1 & $\mid---$ & -------1 & -------- & |--------- & -------- \\
\hline 1 & $14.536 \mathrm{BB}$ & 0.3587 & $2.92708 \mathrm{e} 4$ & 1234.28491 & 49.8016 \\
\hline 2 & $22.707 \mathrm{BB}$ & 0.7902 & $2.95040 \mathrm{e} 4$ & 550.57983 & 50.1984 \\
\hline $\mathrm{tal}$ & Ls: & & $5.87748 \mathrm{e} 4$ & 1784.86475 & \\
\hline
\end{tabular}


Data File D: \DATA \LYH \LYH-4-656-RAC \LY-4-656-RAC-FURAN 2019-04-12 14-40-53\086-2501.D Sample Name: $2 X-3-81-4 M e$

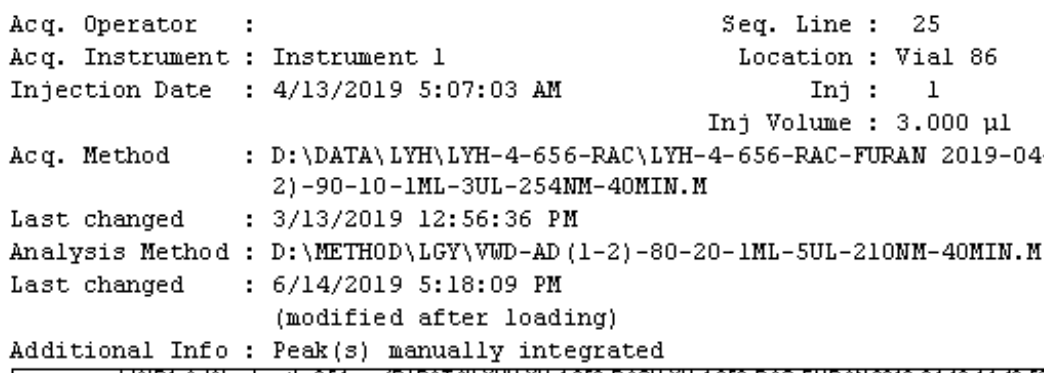
2) $-90-10-1 M L-3 U L-254 M M-40 M I N . M$

Last changed : $3 / 13 / 2019$ 12:56:36 PM

Analysis Method : D: \METHOD LGY VWD-AD (1-2)-80-20-1ML-5UL-210NM-40MIN.M

Last changed : 6/14/2019 5:18:09 PM

(modified after loading)

Additional Info : Peak (s) manually integrated

Ac q. Method : D: \DATA $\backslash$ LYH $\backslash$ LYH-4-656-RAC $\backslash$ LYH-4-656-RAC-FURAN 2019-04-12 14-40-53 VTD-AD (1-

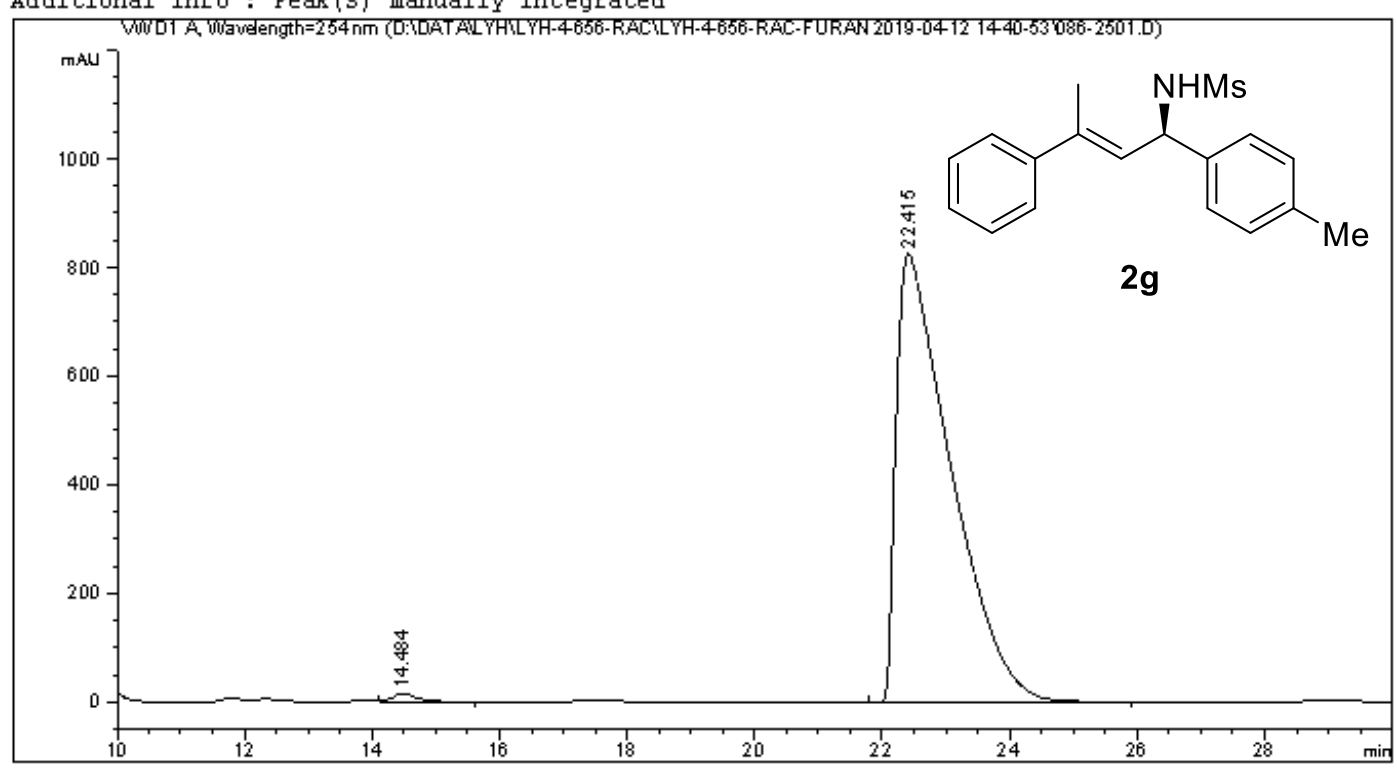

Area Percent Report

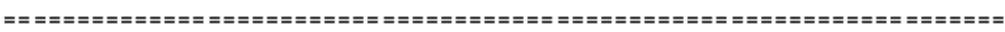

$\begin{array}{lcc}\text { Sorted By } & : & \text { Signal } \\ \text { Multiplier } & : & 1.0000 \\ \text { Dilution } & : & 1.0000 \\ \text { Use Multiplier } & \text { \& Dilution } & \text { Factor with }\end{array}$

Signal 1: VTD 1 , Wavelength=254 nm

\begin{tabular}{|c|c|c|c|c|c|}
\hline $\begin{array}{c}\mathrm{Peak} \\
\#\end{array}$ & $\begin{array}{l}\text { RetTime Type } \\
\text { [min] }\end{array}$ & $\begin{array}{l}\text { Width } \\
\text { [min] }\end{array}$ & $\begin{array}{c}\text { Area } \\
\text { [mAUt*s] }\end{array}$ & $\begin{array}{l}\text { Height } \\
\text { [ṁU] }\end{array}$ & $\begin{array}{c}\text { Area } \\
\stackrel{8}{*}\end{array}$ \\
\hline--1 & $1---$ & -------1 & ------ & ------- & 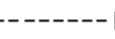 \\
\hline 1 & $14.484 \mathrm{VB}$ & 0.3622 & 363.28226 & 15.07080 & 0.7366 \\
\hline 2 & $22.415 \mathrm{BB}$ & 0.8917 & $4.89580 \mathrm{e} 4$ & 825.75500 & 99.2634 \\
\hline
\end{tabular}

$4.93213 e 4 \quad 840.82581$ 
Data File D: \DATA \LD $\backslash$ LWD-5-14-1 LWD-5-14-1 2019-03-13 10-06-46\019-3601.D

Sample Name: $2 X-3-81-3 F-R A C$

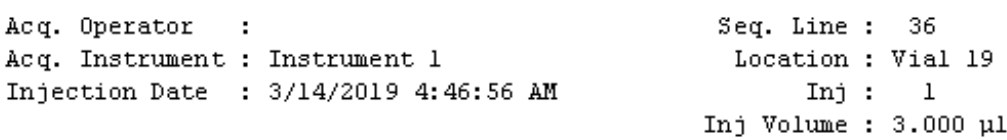

Inj Volume : $3.000 \mu 1$

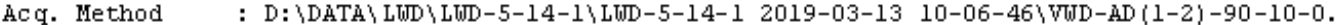
5 ML-3UL-254MM-60MIN. M

Last changed : 3/13/2019 1:08:26 PM

Analysis Method : D: \METHOD LGY VWD-AD (1-2)-80-20-1ML-5UL-210NM-40MIN. M

Last changed : 6/14/2019 5:08:13 PM

(modified after loading)

Additional Info : Peak (s) manually integrated

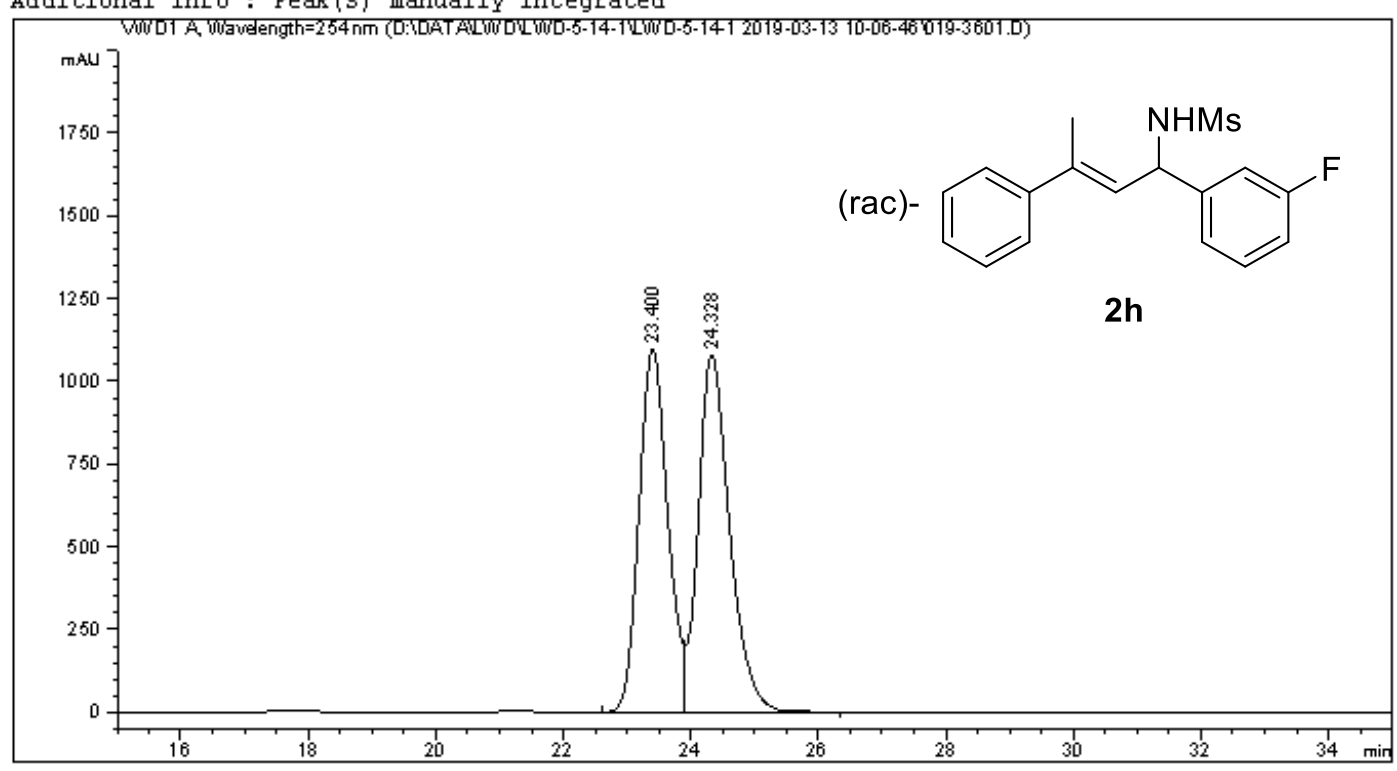

Area Percent Report

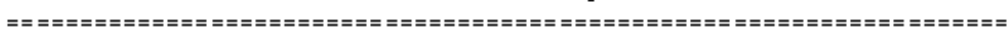

$\begin{array}{lcc}\text { Sorted By } & : & \text { Signal } \\ \text { Multiplier } & : & 1.0000 \\ \text { Dilution } & : & 1.0000 \\ \text { Use Multiplier } & \text { \& Dilution Factor with ISTDs }\end{array}$

signal 1: VWDl A, Wavelength=254 nm

\begin{tabular}{|c|c|c|c|c|c|}
\hline $\begin{array}{c}\text { Peak } \\
\#\end{array}$ & $\begin{array}{l}\text { RetTime Type } \\
\text { [min] }\end{array}$ & $\begin{array}{l}\text { Width } \\
\text { [min] }\end{array}$ & $\begin{array}{c}\text { Area } \\
\text { [mAUt's] }\end{array}$ & 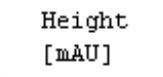 & $\begin{array}{c}\text { Area } \\
\stackrel{8}{*}\end{array}$ \\
\hline- & ------1 & & -------- & |--------- & ------ \\
\hline 1 & $23.400 \mathrm{VV}$ & 0.4766 & $3.43375 \mathrm{e} 4$ & 1098.75171 & 48.2428 \\
\hline 2 & 24.328 VB & 0.5119 & $3.68390 \mathrm{e} 4$ & 1080.26965 & 51.7572 \\
\hline ota & : & & $.11765 \mathrm{e} 4$ & 179.02136 & \\
\hline
\end{tabular}


Data File D: \DATA $\backslash L D \backslash L W D-5-14-1 \backslash L$ LD-5-14- 1 2019-03-13 10-06-46\020-3701.D

Sample Name: $2 X-3-83-3 F$

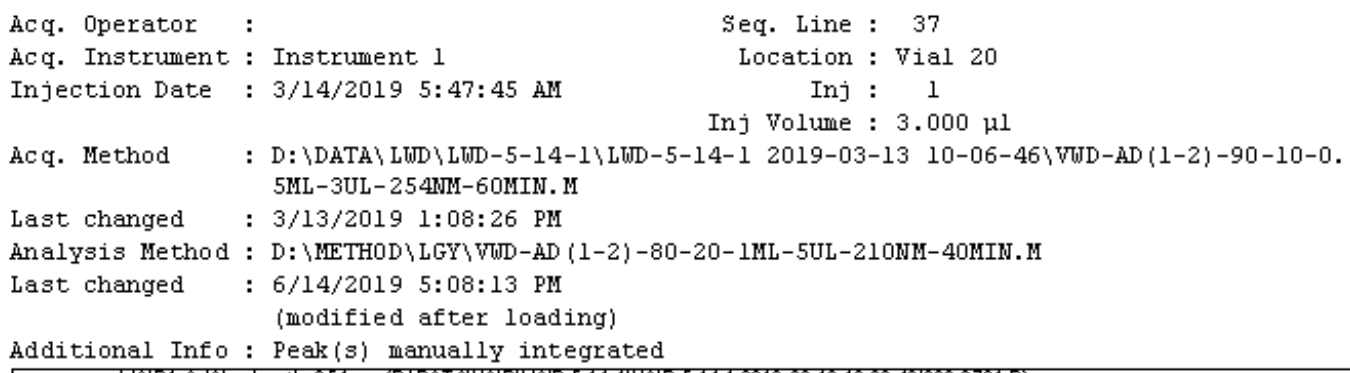

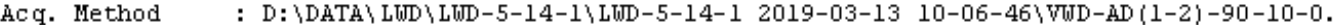
$5 \mathrm{ML}-3 \mathrm{UL}-254 \mathrm{MM}-60 \mathrm{MIN}$. M

Last changed : 3/13/2019 1:08:26 PM

Analysis Method : D: \METHOD LGY VWD-AD (1-2)-80-20-1ML-5UL-210NM-40MIN.M

Last changed : 6/14/2019 5:08:13 PM

(modified after loading)

Additional Info : Peak (s) manually integrated

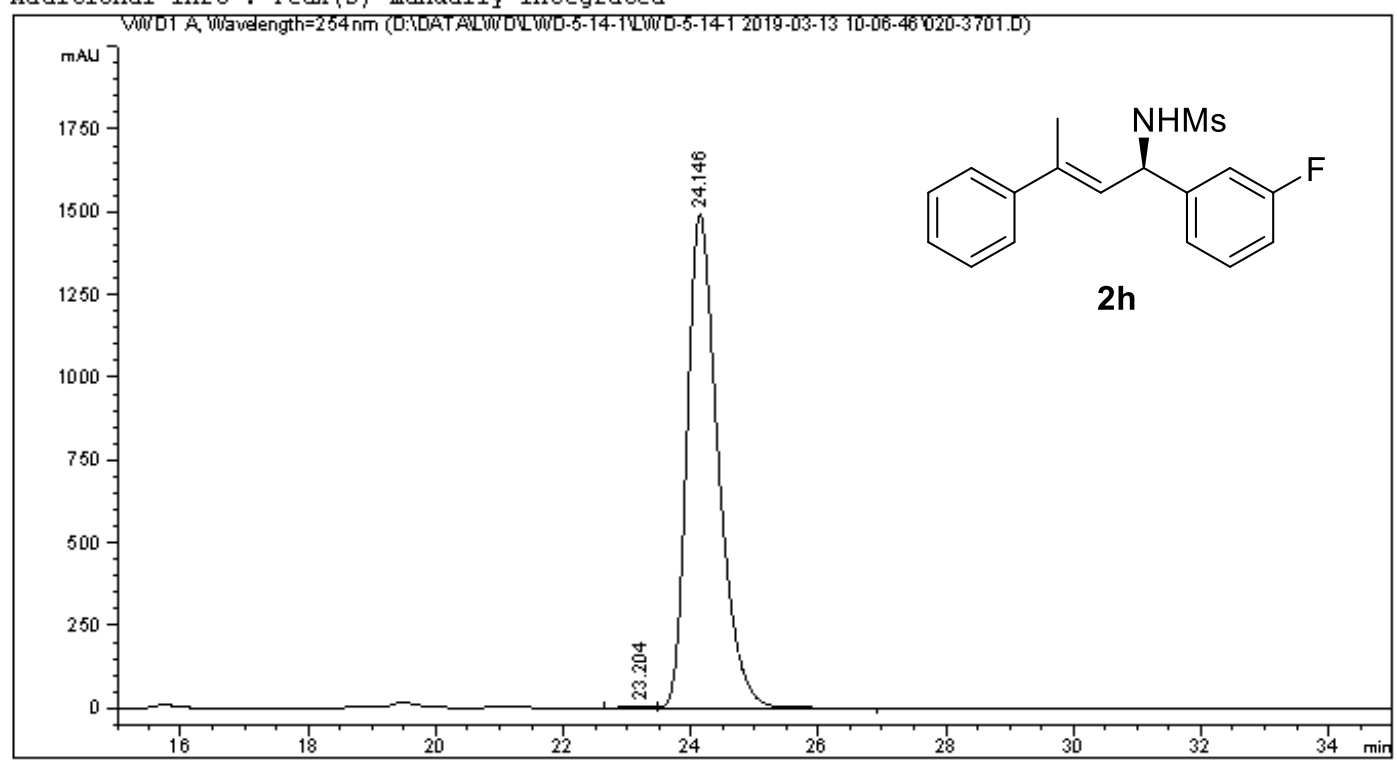

Area Percent Report

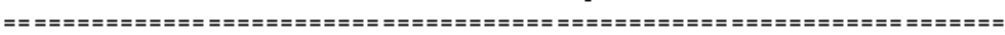

$\begin{array}{lcc}\text { Sorted By } & : & \text { Signal } \\ \text { Multiplier } & : & 1.0000 \\ \text { Dilution } & : & 1.0000 \\ \text { Use Multiplier } & \text { \& Dilution Factor with }\end{array}$

Signal 1: VTD 1 , Wavelength=254 nm

\begin{tabular}{|c|c|c|c|c|c|}
\hline $\begin{array}{c}\text { Peak } \\
\#\end{array}$ & $\begin{array}{l}\text { RetTime Type } \\
\text { [min] }\end{array}$ & $\begin{array}{l}\text { Width } \\
\text { [min] }\end{array}$ & $\begin{array}{c}\text { Area } \\
\text { [mAUt's] }\end{array}$ & $\begin{array}{l}\text { Height } \\
\text { [midu] }\end{array}$ & $\begin{array}{c}\text { Area } \\
\stackrel{8}{8}\end{array}$ \\
\hline- & -----1 & ------1 & ------ & ---------- & --- \\
\hline 1 & $23.204 \mathrm{BV}$ & 0.4140 & 196.48953 & 7.39501 & 0.3868 \\
\hline 2 & 24.146 VB & 0.5158 & $5.06002 \mathrm{e} 4$ & 1491.66187 & 99.6132 \\
\hline ota & : & & $07966 \mathrm{e} 4$ & 499.05688 & \\
\hline
\end{tabular}


Data File D: \DATA $\backslash L D \backslash L W D-5-14-1 \backslash L$ LD-5-14- 1 2019-03-13 10-06-46\021-3801.D Sample Name: $2 X-3-81-30 \mathrm{Me}-\mathrm{RAC}$

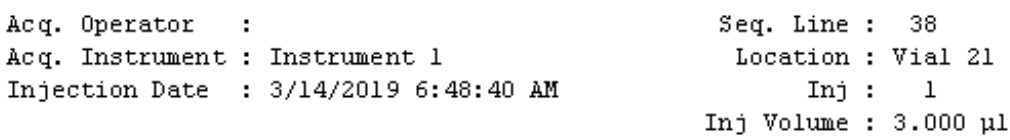

Ac q. Method : D: \DATA \LDD LUDD-5-14-1 LUDD-5-14-1 2019-03-13 10-06-46 VVID-AD (1-2) -90-10-1ML $-3 \mathrm{UL}-254 \mathrm{MM}-40 \mathrm{MIN}$. M

Last changed : $3 / 13 / 2019$ 12:56:36 PM

Analysis Method : D: \METHOD LGY VWD-AD (1-2)-80-20-1ML-5UL-210NM-40MIN. M

Last changed : 6/14/2019 5:05:36 PM

(modified after loading)

Additional Info : Peak (s) manually integrated

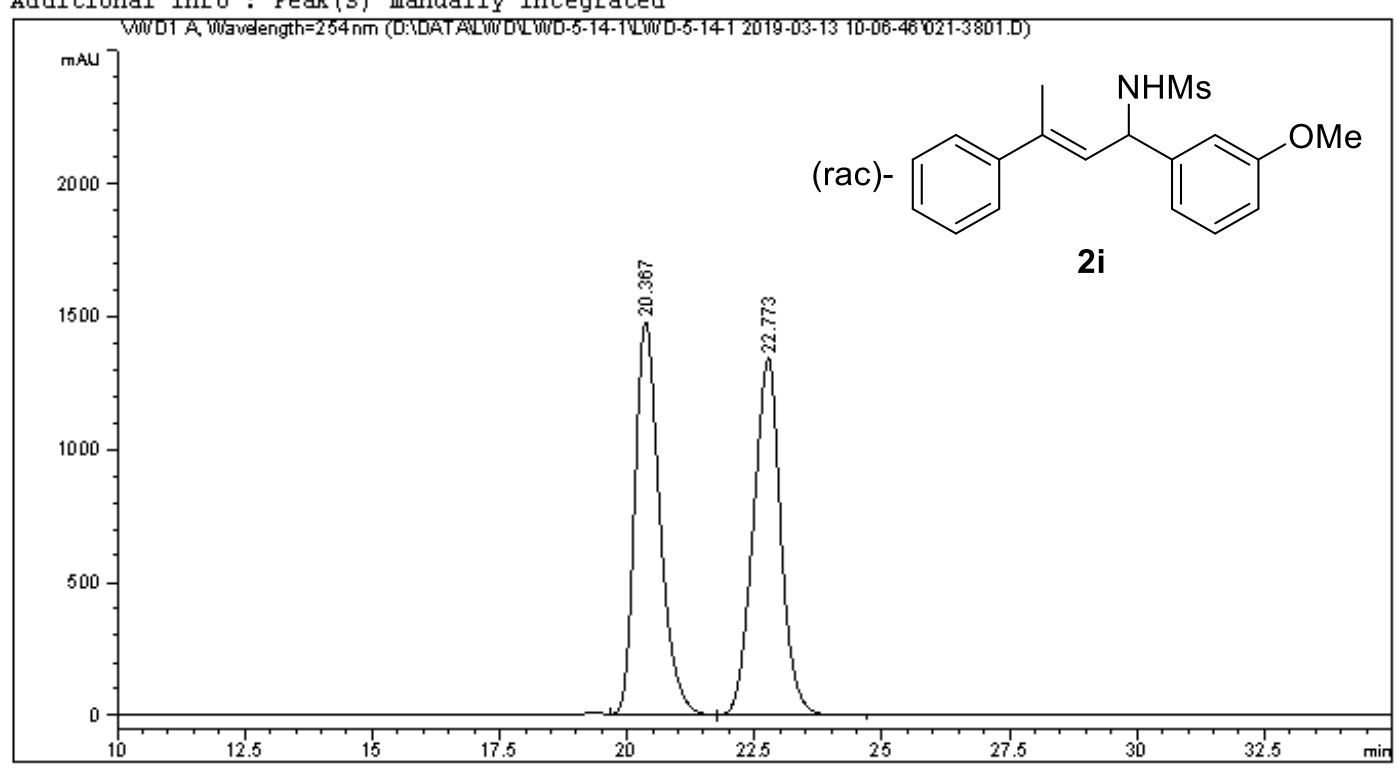

Area Percent Report

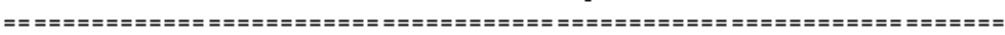

$\begin{array}{lcc}\text { Sorted By } & : & \text { Signal } \\ \text { Multiplier } & : & 1.0000 \\ \text { Dilution } & : & 1.0000 \\ \text { Use Multiplier } & \text { \& Dilution Factor with ISTDs }\end{array}$

Signal 1: VWDl A, Wavelength=254 nm

\begin{tabular}{|c|c|c|c|c|c|c|}
\hline $\begin{array}{c}\text { Peak } \\
\#\end{array}$ & $\begin{array}{c}\text { RetTime } \\
\text { [min] }\end{array}$ & Type & $\begin{array}{l}\text { Width } \\
\text { [min] }\end{array}$ & $\begin{array}{c}\text { Area } \\
\text { [mAUt*s] }\end{array}$ & $\begin{array}{l}\text { Height } \\
\text { [minu] }\end{array}$ & $\begin{array}{c}\text { Area } \\
\stackrel{4}{*}\end{array}$ \\
\hline-- & ----- & & $-------\mid$ & --------- & | -------- & -------1 \\
\hline 1 & 20.367 & VB & 0.5182 & $5.01822 \mathrm{e} 4$ & 1477.62708 & 49.9807 \\
\hline 2 & 22.773 & $\mathrm{BB}$ & 0.5709 & $5.02209 \mathrm{e} 4$ & 1341.09204 & 50.0193 \\
\hline \multicolumn{4}{|c|}{ Totals: } & $1.00403 \mathrm{e} 5$ & 2818.71912 & \\
\hline
\end{tabular}


Data File D: \DATA $\backslash L D \backslash L W D-5-14-1 \backslash L$ LD-5-14- 1 2019-03-13 10-06-46\022-3901.D

Sample Name: $2 X-3-81-30 \mathrm{Me}$

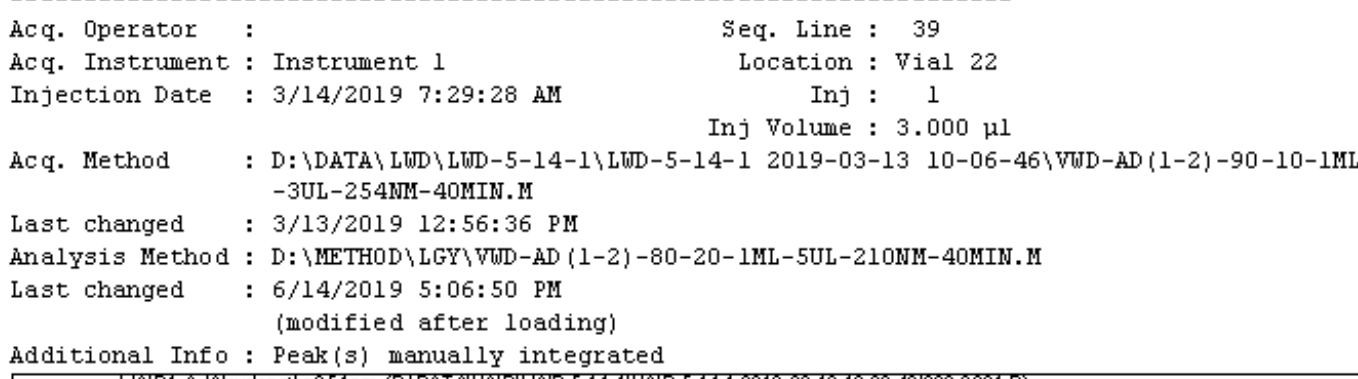

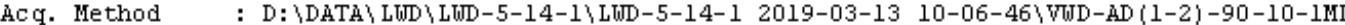
$-3 \mathrm{UL}-254 \mathrm{MM}-40 \mathrm{MIN} . \mathrm{M}$

Last changed : $3 / 13 / 2019$ 12:56:36 PM

Analysis Method : D: \METHOD LGY VWD-AD (1-2)-80-20-1ML-5UL-210NM-40MIN. M

Last changed : 6/14/2019 5:06:50 PM

(modified after loading)

Additional Info : Peak (s) manually integrated

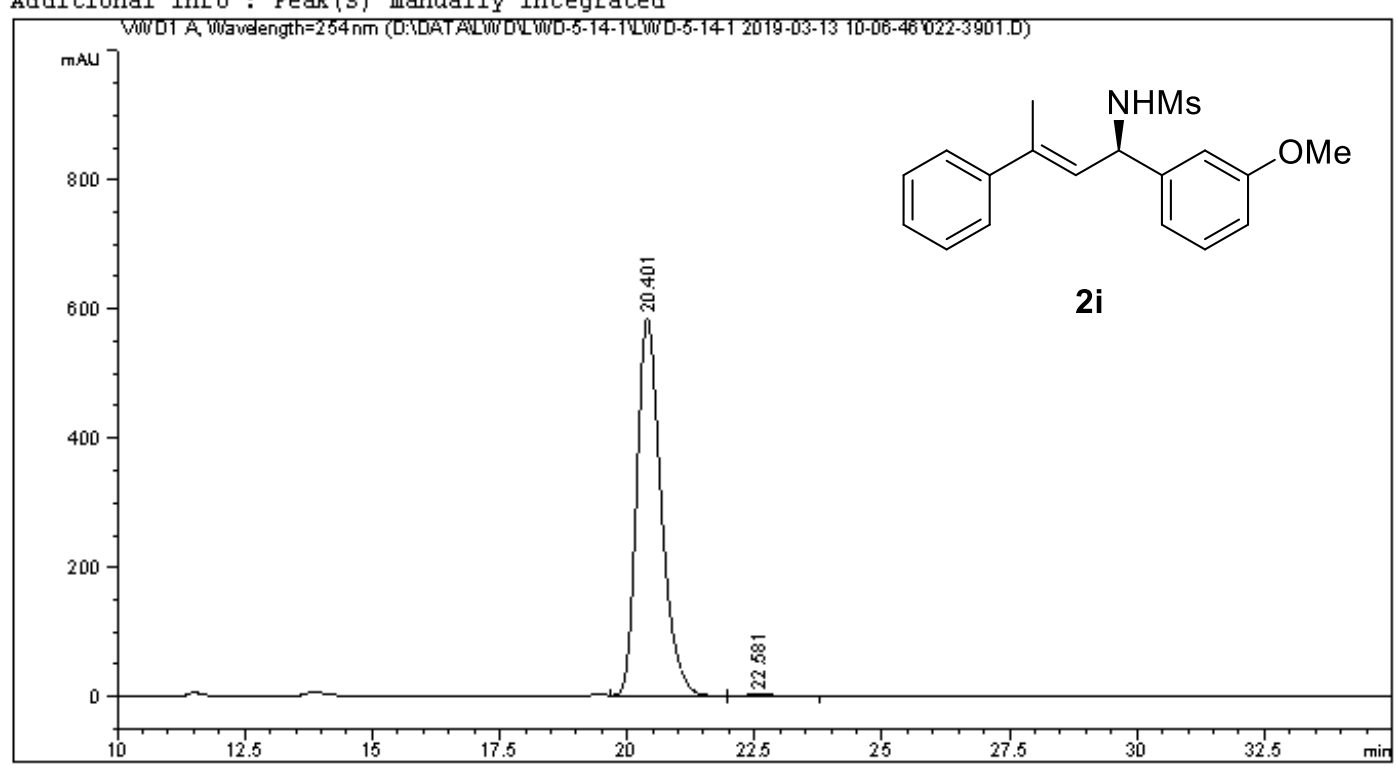

Area Percent Report

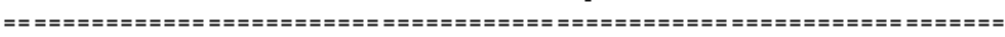

$\begin{array}{lcc}\text { Sorted By } & : & \text { Signal } \\ \text { Multiplier } & : & 1.0000 \\ \text { Dilution } & : & 1.0000 \\ \text { Use Multiplier } & \text { \& Dilution } & \text { Factor with }\end{array}$

Signal 1: VWDl A, Wavelength=254 nm

\begin{tabular}{|c|c|c|c|c|c|}
\hline $\begin{array}{c}\text { eak } \\
\#\end{array}$ & $\begin{array}{l}\text { RetTime Type } \\
\text { [min] }\end{array}$ & $\begin{array}{l}\text { Width } \\
\text { [min] }\end{array}$ & $\begin{array}{c}\text { Area } \\
\text { [mÂU*'s ] }\end{array}$ & $\begin{array}{l}\text { Height } \\
\text { [mAU] }\end{array}$ & $\begin{array}{c}\text { Area } \\
\stackrel{5}{*}\end{array}$ \\
\hline & & & -- & & - \\
\hline 1 & $B_{1}$ & 0 & 1. $94961 \mathrm{e} 4$ & 4291 & 246 \\
\hline 2 & $1 \mathrm{BB}$ & 0.5405 & 93.13364 & 2.62166 & 0.4754 \\
\hline
\end{tabular}

Totals : $\quad 1.95892 \mathrm{e} 4 \quad 587.66457$ 
Data File D: \DATA $\backslash X \backslash Z X-3-81 \backslash L S L-4-31-3 \quad 2019-04-13$ 13-34-06 011-0301.D Sample Name: $2 X-3-81-41$ ian-RAX
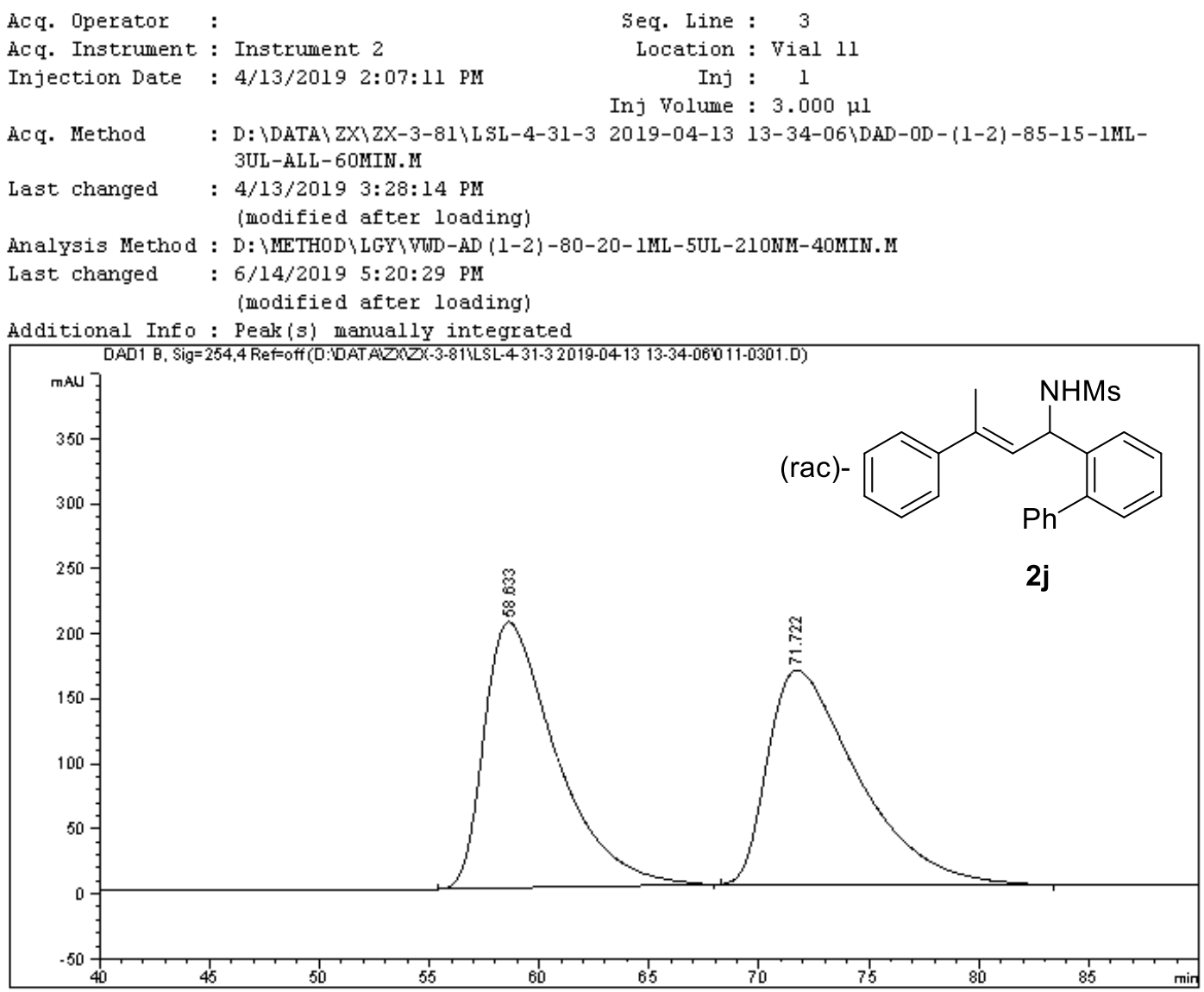

\section{Area Percent Report}

\begin{tabular}{|c|c|c|}
\hline Sorted BY & : & Signal \\
\hline Multiplier & $:$ & 1.0000 \\
\hline Dilution & : & 1.0000 \\
\hline
\end{tabular}

Use Multiplier \& Dilution Factor with IsTDs

Signal 1: DADl B, Sig=254, 4 Ref=off

\begin{tabular}{|c|c|c|c|c|c|}
\hline $\begin{array}{c}\text { Peak } \\
\#\end{array}$ & $\begin{array}{l}\text { RetTime Type } \\
\text { [min] }\end{array}$ & $\begin{array}{l}\text { Width } \\
\text { [min] }\end{array}$ & $\begin{array}{c}\text { Area } \\
{\left[\mathrm{m} \mathrm{AU}^{*} \mathrm{~s}\right]}\end{array}$ & $\begin{array}{l}\text { Height } \\
\text { [MHU] }\end{array}$ & Àrea \\
\hline 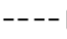 & $------\mid--$ & ------- & --------- & ------- & -------1 \\
\hline 1 & $58.633 \mathrm{BB}$ & 2.6722 & 4. $67655 \mathrm{e} 4$ & 204.63832 & 49.9267 \\
\hline 2 & $71.722 \mathrm{BB}$ & 3.3276 & $4.69027 \mathrm{e} 4$ & 164.73854 & 50.0733 \\
\hline Tota. & : & & $9.36682 \mathrm{e} 4$ & 369.37686 & \\
\hline
\end{tabular}


Data File D: \DATA $\backslash X \backslash Z X-3-81 \backslash L S L-4-31-3 \quad 2019-04-13 \quad 13-34-06 \backslash 012-0401 . D$

Sample Name: $2 X-3-81-41$ ian

Acq. Operator :

Ac q. Instrument : Instrument 2

Injection Date : 4/13/2019 3:38:07 PM

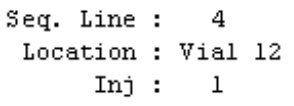

Seq. Line : 4

Location : Vial 12

Inj : 1

Inj Volume : $3.000 \mu 1$

Ac q. Method : D: \DATA $\backslash Z X \backslash Z X-3-81 \backslash$ L SL-4-31-3 2019-04-13 13-34-06 DAD-0D-(1-2)-85-15-1ML3UL-ALL-60MIN. M

Last changed : 4/13/2019 3:28:14 PM

(modified after loading)

Analysis Method : D: \METHOD LGY \VTD-AD (1-2)-80-20-1ML-5UL-210NM-40MIN. M

Last changed : 6/14/2019 5:22:18 PM

(modified after loading)

Additional Info: Peak (s) manually integrated

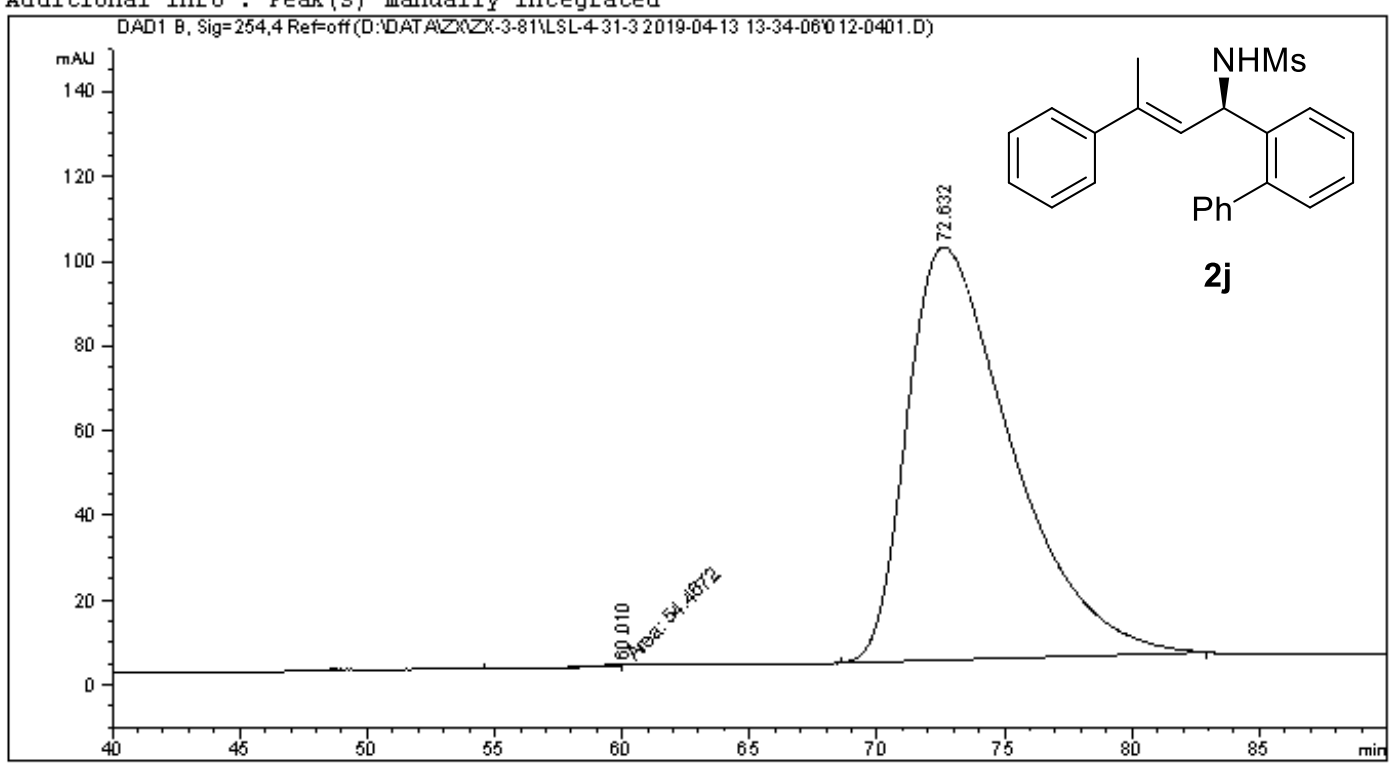

\section{Area Percent Report}

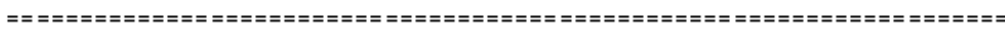

$\begin{array}{lll}\text { Sorted By } & : & \text { Signal } \\ \text { Multiplier } & : & 1.0000 \\ \text { Dilution } & : & 1.0000\end{array}$

Use Multiplier \& Dilution Factor with IsTDs

Signal 1: DADl B, Sig=254, 4 Ref=off

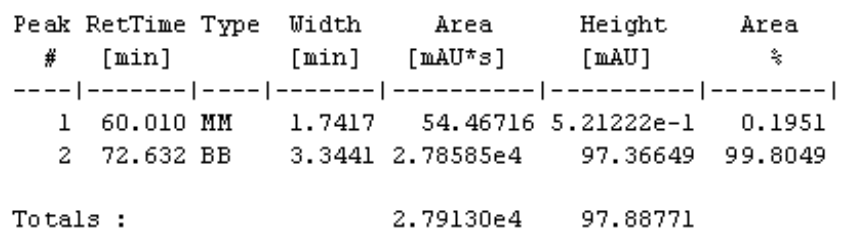


Data File D: \DATA $\backslash$ LYH $\backslash$ LYH-4-757 LYH-4-757-RAC 2019-05-26 20-13-55 073-0601.D Sample Name: ZX-3-8l-4'Me-RAC
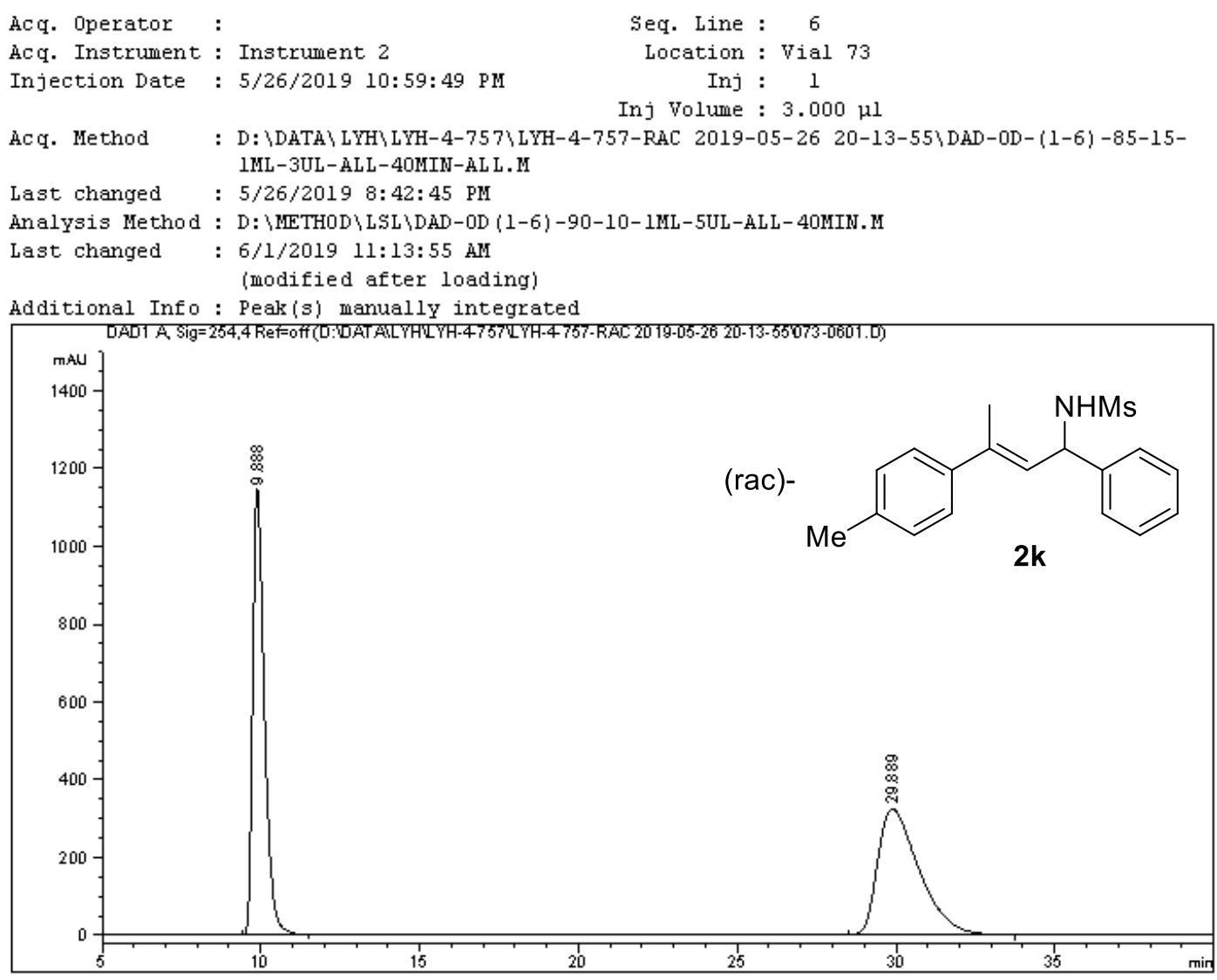

Area Percent Report

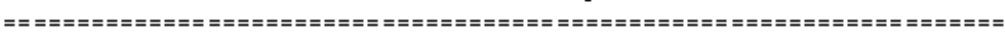

$\begin{array}{lll}\text { Sorted BY } & : & \text { Signal } \\ \text { Multiplier } & : & 1.0000 \\ \text { Dilution } & : & 1.0000\end{array}$

Use Multiplier \& Dilution Factor with ISTDs

Signal 1: DADl A, Sig=254,4 Ref=off

\begin{tabular}{|c|c|c|c|c|c|c|}
\hline $\begin{array}{c}\mathrm{Peak} \\
\#\end{array}$ & $\begin{array}{c}\text { RetTime } \\
\text { [min] }\end{array}$ & Type & $\begin{array}{l}\text { Width } \\
\text { [min] }\end{array}$ & $\begin{array}{c}\text { Area } \\
{\left[\mathrm{m} A U^{*} S\right]}\end{array}$ & $\begin{array}{l}\text { Height } \\
\text { [mAU] }\end{array}$ & $\begin{array}{c}\text { Area } \\
\stackrel{8}{*}\end{array}$ \\
\hline-- & & & 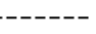 & -------1 & 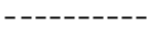 & $-1--1$ \\
\hline 1 & 9.888 & $\mathrm{BB}$ & 0.3870 & $2.91401 \mathrm{e} 4$ & 1144.56738 & 49.9205 \\
\hline 2 & 29.889 & $\mathrm{BB}$ & 1.2623 & $2.92330 \mathrm{e} 4$ & 322.85999 & 50.0795 \\
\hline Tote & : & & & $5.83730 \mathrm{e} 4$ & 1467.42737 & \\
\hline
\end{tabular}


Data File D: \DATA $\backslash$ LYH $\backslash$ LYH-4-757 LYH-4-757-RAC 2019-05-26 20-13-55\074-0701.D Sample Name: $2 X-3-81-4$ 'Me
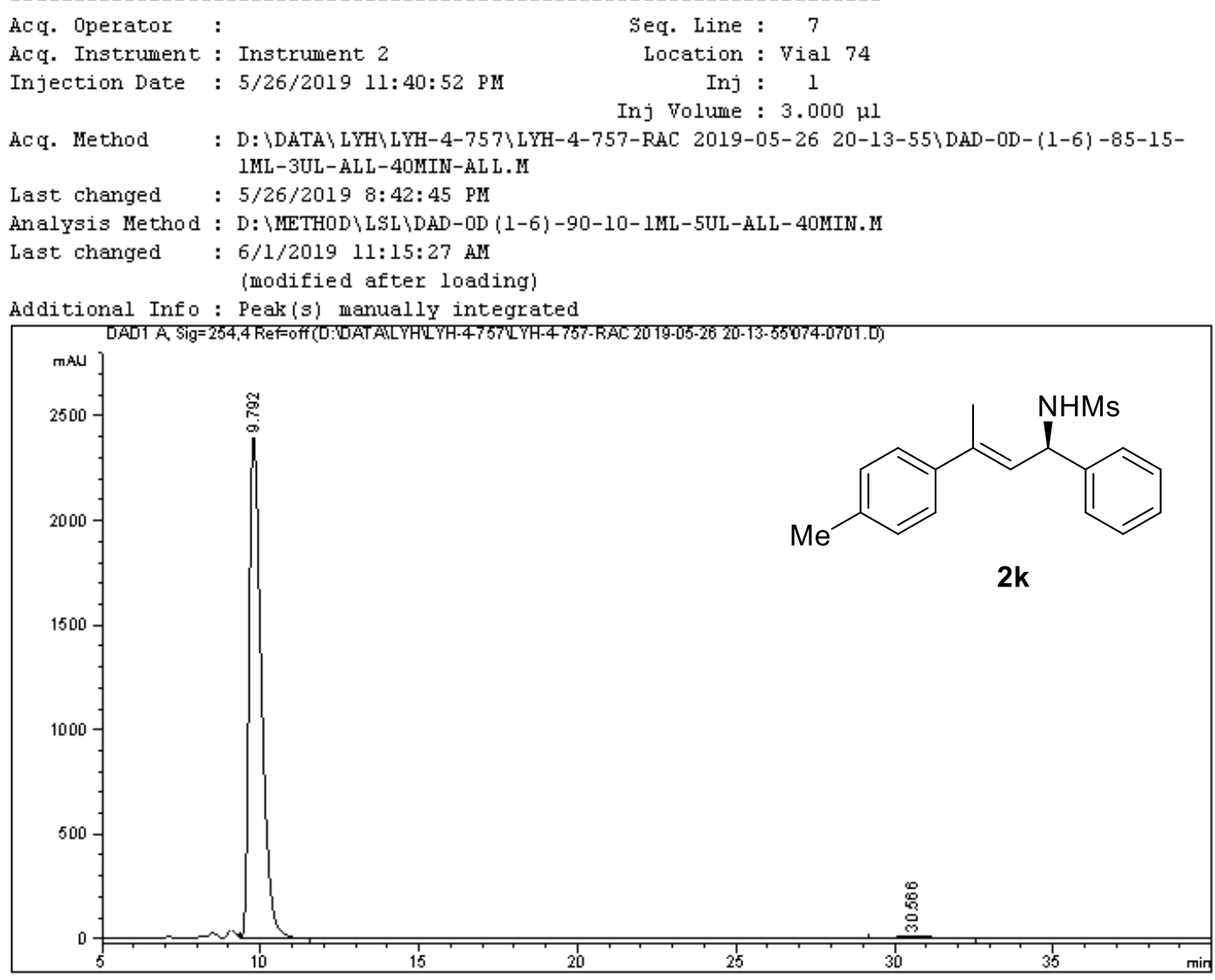

Area Percent Report

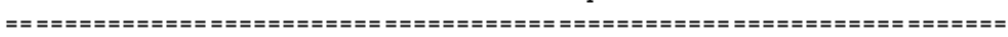

$\begin{array}{lll}\text { Sorted By } & : & \text { signal } \\ \text { Multiplier } & : & 1.0000 \\ \text { Dilution } & : & 1.0000\end{array}$

Use Multiplier \& Dilution Factor with ISTDs

Signal 1: DADl A, Sig=254, 4 Ref=off

\begin{tabular}{|c|c|c|c|c|c|c|}
\hline $\begin{array}{c}\mathrm{Peak} \\
\#\end{array}$ & $\begin{array}{l}\text { RetTime } \\
\text { [min] }\end{array}$ & Type & $\begin{array}{l}\text { Width } \\
\text { [min] }\end{array}$ & $\begin{array}{c}\text { Area } \\
{\left[\mathrm{m} A U^{*} S\right]}\end{array}$ & $\begin{array}{l}\text { Height } \\
\text { [mAU] }\end{array}$ & $\begin{array}{c}\text { Area } \\
\stackrel{8}{*}\end{array}$ \\
\hline-- & & & --- & --- & $-0-0-1$ & 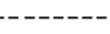 \\
\hline 1 & 9.792 & VB & 0.3305 & $6.44699 \mathrm{e} 4$ & 2391.26782 & 98.4760 \\
\hline 2 & 30.566 & $\mathrm{BB}$ & 0.9872 & 997.73560 & 11.93662 & 1.5240 \\
\hline Tote & : : & & & $6.54676 \mathrm{e} 4$ & 2403.20444 & \\
\hline
\end{tabular}


Data File D: \DATA $\backslash$ LYH $\backslash$ LYH-4-757 LYH-4-757-RAC 2019-05-26 20-13-55\075-0901.D Sample Name: $2 X-3-81-4 ' C l-R A C$
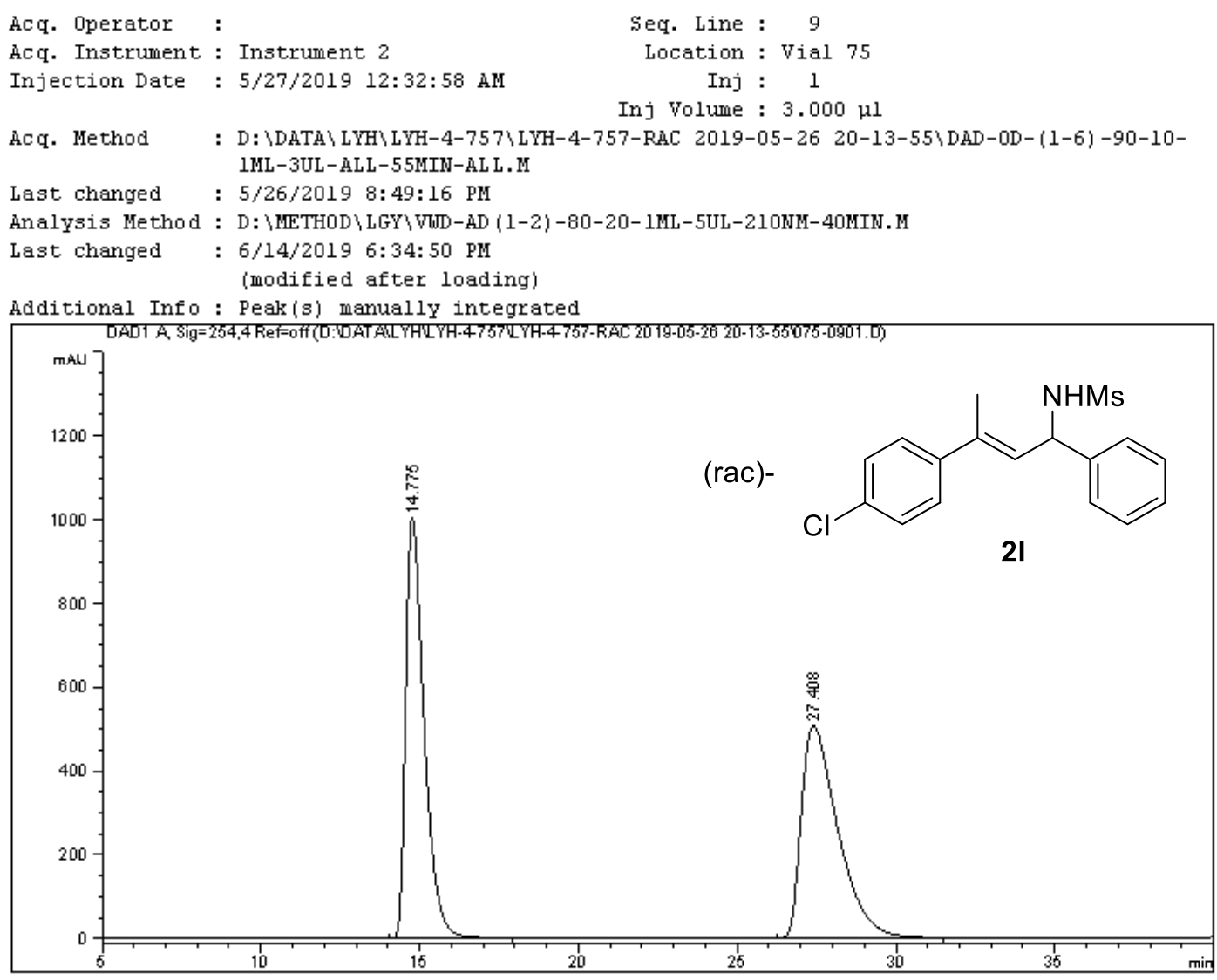

Area Percent Report

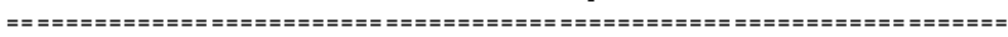

$\begin{array}{lll}\text { Sorted By } & : & \text { signal } \\ \text { Multiplier } & : & 1.0000 \\ \text { Dilution } & : & 1.0000\end{array}$

Use Multiplier \& Dilution Factor with ISTDs

Signal 1: DADl A, Sig=254, 4 Ref=off

\begin{tabular}{|c|c|c|c|c|c|c|}
\hline $\begin{array}{c}\text { Peak } \\
\#\end{array}$ & $\begin{array}{c}\text { RetTime } \\
\text { [min] }\end{array}$ & Type & $\begin{array}{l}\text { Width } \\
\text { [min] }\end{array}$ & 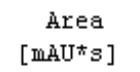 & $\begin{array}{l}\text { Height } \\
\text { [mAU] }\end{array}$ & $\begin{array}{c}\text { Area } \\
\text { \& }\end{array}$ \\
\hline-- & & & 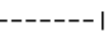 & $----1--1$ & 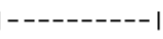 & - \\
\hline 1 & 14.775 & $\mathrm{BB}$ & 0.6098 & $4.03263 \mathrm{e} 4$ & 1001.41882 & 49.9539 \\
\hline 2 & 27.408 & $\mathrm{BB}$ & 1.1531 & $4.04007 \mathrm{e} 4$ & 506.23544 & 50.0461 \\
\hline Tota & ls: & & & $8.07271 \mathrm{e} 4$ & 1507.65427 & \\
\hline
\end{tabular}


Data File D: \DATA $\backslash$ LYH $\backslash$ LYH-4-757 LYH-4-757-RAC 2019-05-26 20-13-55\076-1001.D Sample Name: $2 X-3-81-4^{\prime} \mathrm{Cl}$
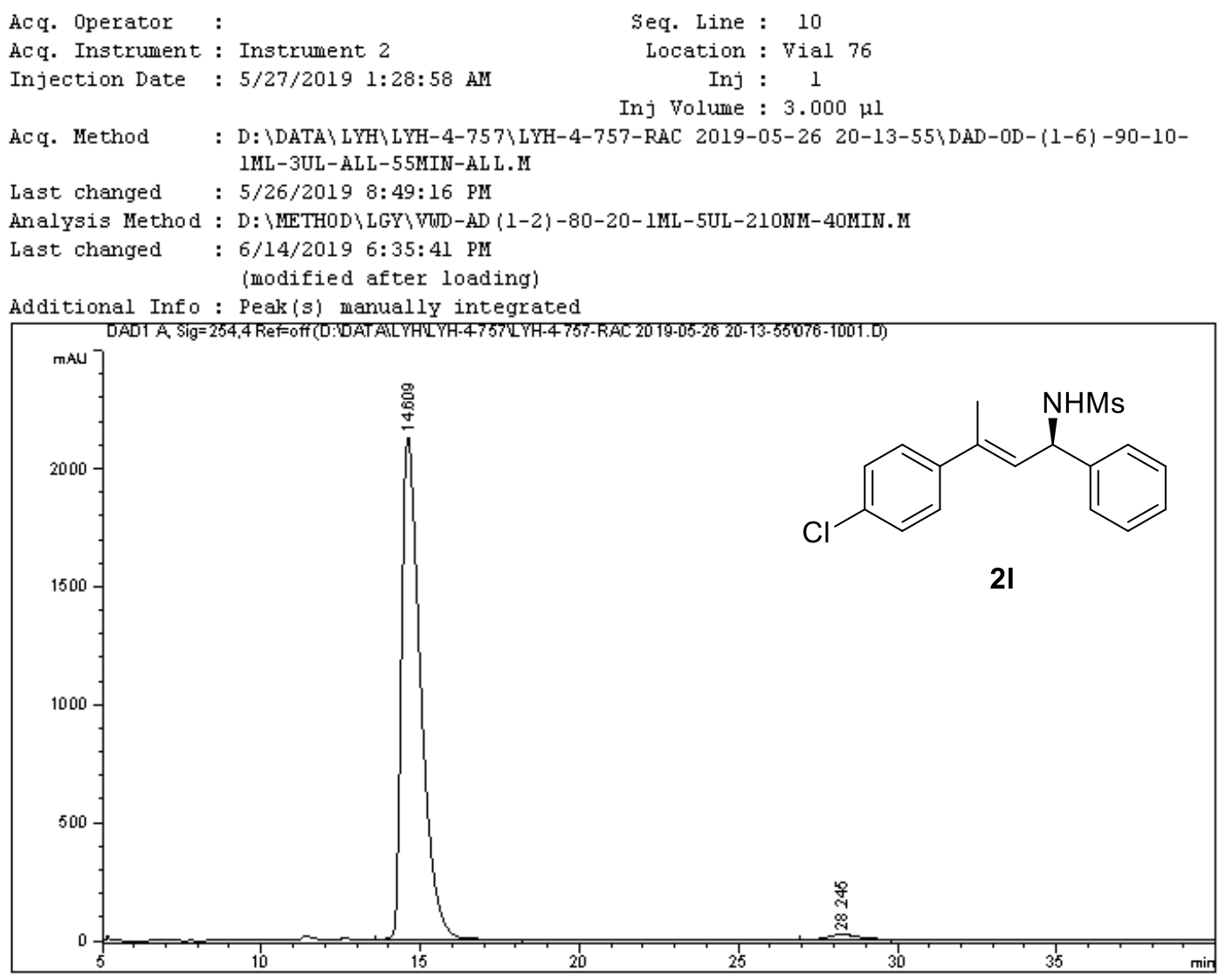

Area Percent Report

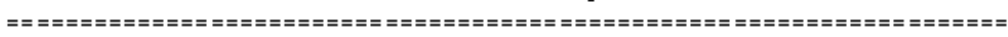

$\begin{array}{lll}\text { Sorted By } & : & \text { signal } \\ \text { Multiplier } & : & 1.0000 \\ \text { Dilution } & : & 1.0000\end{array}$

Use Multiplier \& Dilution Factor with ISTDs

Signal 1: DADl A, Sig=254, 4 Ref=off

\begin{tabular}{|c|c|c|c|c|c|c|}
\hline $\begin{array}{c}\mathrm{Peak} \\
\#\end{array}$ & $\begin{array}{c}\text { RetTime } \\
\text { [min] }\end{array}$ & Type & $\begin{array}{l}\text { Width } \\
\text { [min] }\end{array}$ & $\begin{array}{c}\text { Area } \\
{\left[\mathrm{m} A U^{*} S\right]}\end{array}$ & $\begin{array}{l}\text { Height } \\
\text { [mAU] }\end{array}$ & $\begin{array}{c}\text { Area } \\
\stackrel{8}{*}\end{array}$ \\
\hline-- & & & ----- & -------- & -------- & ---- \\
\hline 1 & 14.609 & $\mathrm{BB}$ & 0.4935 & $8.91190 \mathrm{e} 4$ & 2132.73438 & 98.3296 \\
\hline 2 & 28.245 & $\mathrm{BB}$ & 0.8493 & 1513.95862 & 21.50651 & 1.6704 \\
\hline Tote & Ls: & & & $9.06329 \mathrm{e} 4$ & 2154.24089 & \\
\hline
\end{tabular}


Data File D: \DATA YANG JIAXIN YJX-2-170 YJX-2-170 2019-06-12 12-33-29\075-1801.D Sample Name: zx-3-83-nai-RAC

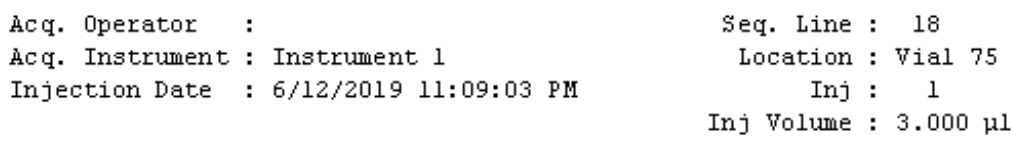

Ac q. Method : D: \DATA YANG JIAXIN YJX-2-170 YJX-2-170 2019-06-12 12-33-29 DAD-AD(1-2)-90$10-1 \mathrm{ML}-3 \mathrm{UL}-\mathrm{ALL}-40 \mathrm{MIN}$. M

Last changed : 6/12/2019 9:46:37 PM

(modified after loading)

Analysis Method : D: \METHOD LGY VWD-AD (1-2)-80-20-1ML-5UL-210NM-40MIN. M

Last changed : 6/14/2019 6:29:46 PM

(modified after loading)

Additional Info: Peak (s) manually integrated

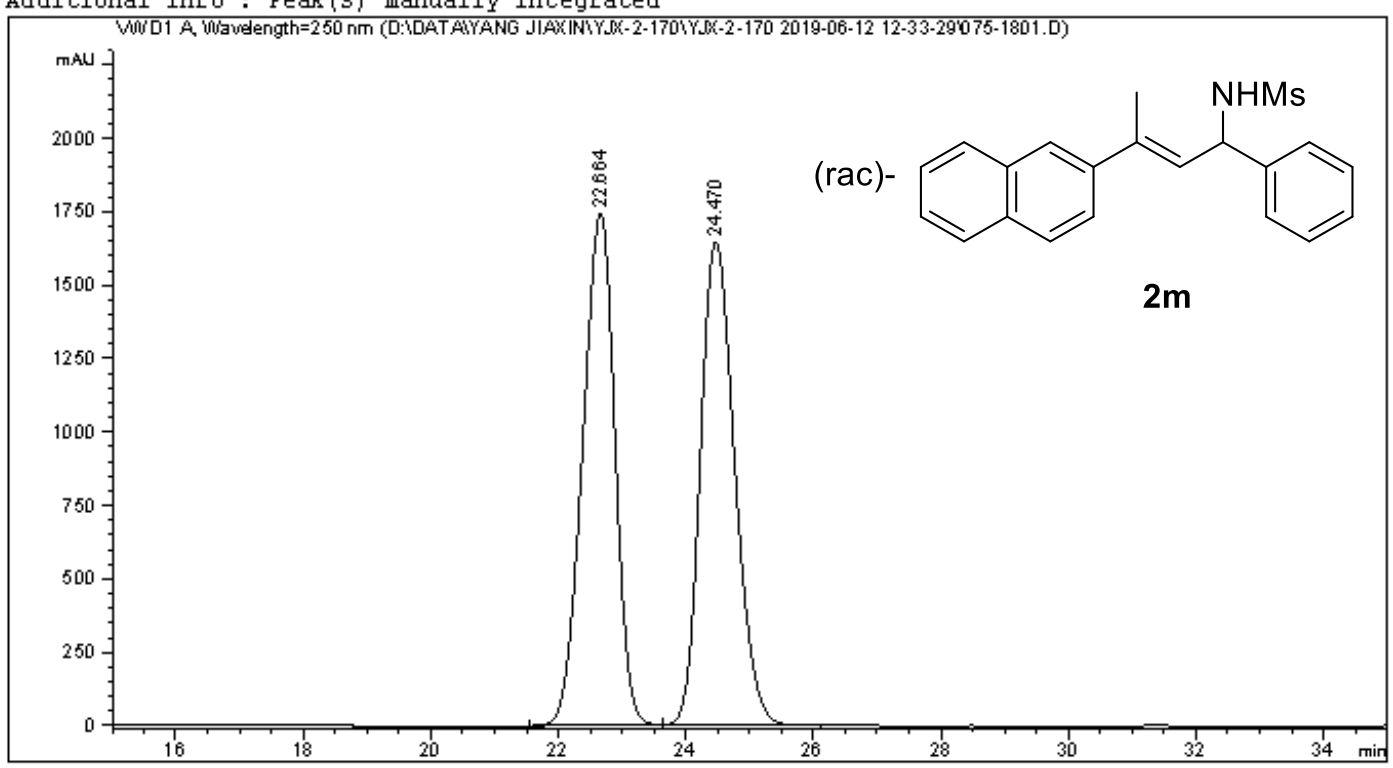

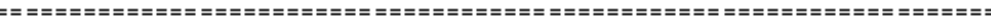

Area Percent Report

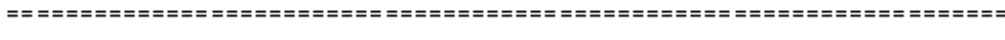

$\begin{array}{lll}\text { Sorted By } & : & \text { Signal } \\ \text { Multiplier } & : & 1.0000 \\ \text { Dilution } & : & 1.0000\end{array}$

Use Multiplier \& Dilution Factor with IsTDs

Signal 1: VWDl A, Wavelength=250 nm

\begin{tabular}{|c|c|c|c|c|c|}
\hline $\begin{array}{c}\text { Peak } \\
\#\end{array}$ & $\begin{array}{l}\text { RetTime Type } \\
\text { [min] }\end{array}$ & $\begin{array}{l}\text { Width } \\
\text { [min] }\end{array}$ & $\begin{array}{c}\text { Area } \\
{\left[\mathrm{m} \mathrm{AU}^{*} \mathrm{~s}\right]}\end{array}$ & $\begin{array}{l}\text { Height } \\
\text { [MAUU] }\end{array}$ & Àrea \\
\hline 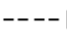 & $------1--$ & -------1 & --------- & |---------- & --------1 \\
\hline 1 & $22.664 \mathrm{BB}$ & 0.5510 & $6.10417 \mathrm{e} 4$ & 1741.29834 & 49.9178 \\
\hline 2 & $24.470 \mathrm{BB}$ & 0.5812 & $6.12428 \mathrm{e} 4$ & 1645.73938 & 50.0822 \\
\hline \multicolumn{3}{|c|}{ Totals : } & 1. $22285 \mathrm{e} 5$ & 3387.03772 & \\
\hline
\end{tabular}


Data File D: \DATA YANG JIAXIN YJX-2-170 YJX-2-170 2019-06-12 12-33-29\076-1901.D Sample Name: zx-3-83-nai

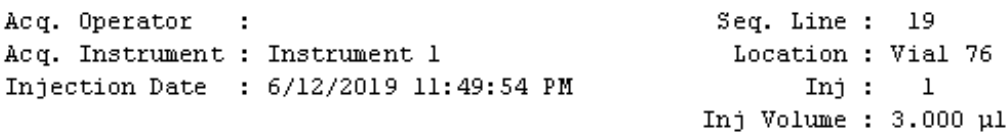

Acq. Method : D: \DATA YANG JIAXIN YJX-2-170 \JX-2-170 2019-06-12 12-33-29 DAD-AD (1-2)-9010-1ML-3UL-ALL-40MIN. M

Last changed : 6/12/2019 9:46:37 PM

(modified after loading)

Analysis Method : D: \METHOD $\backslash$ LGY $\backslash$ VWD-AD (1-2)-80-20-1ML-5UL-210NM-40MIN. M

Last changed : 6/14/2019 6:30:55 PM

(modified after loading)

Additional Info: Peak (s) manually integrated

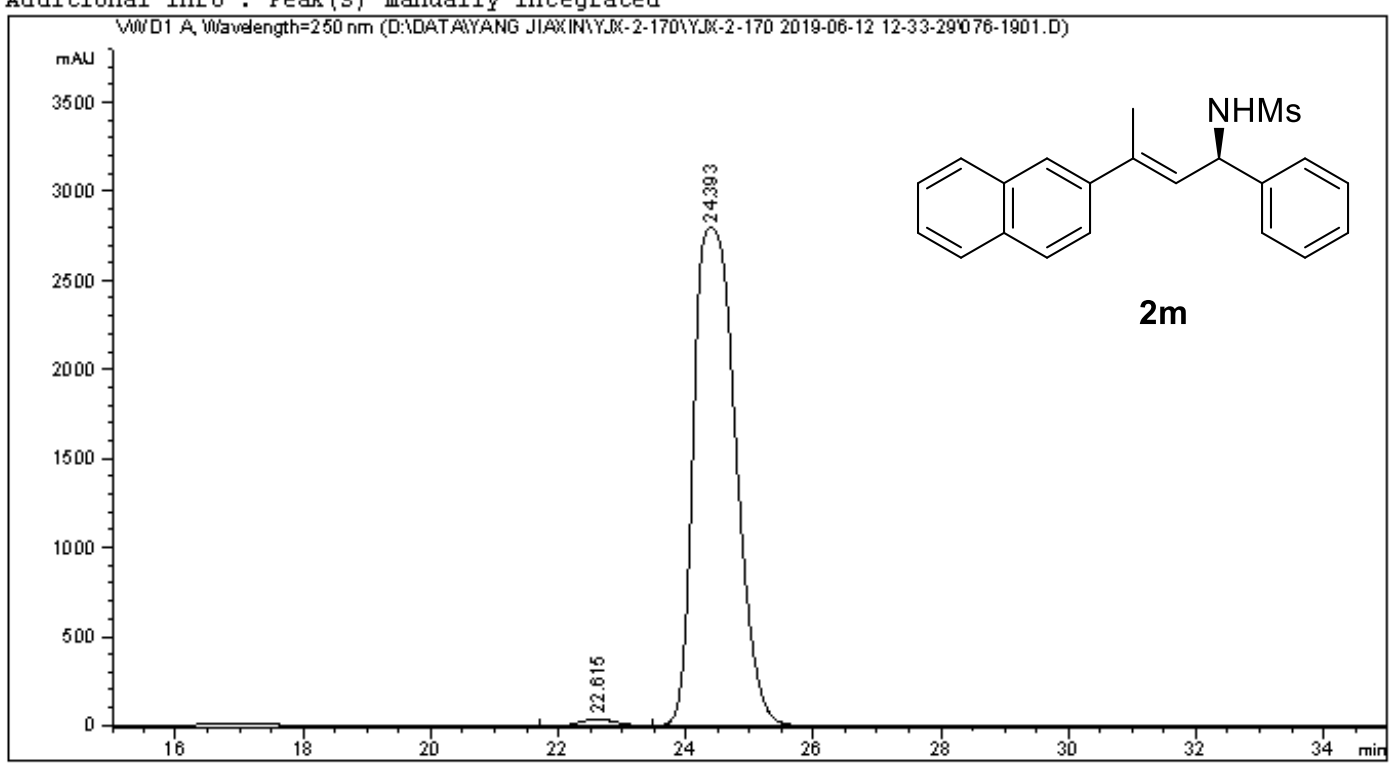

\begin{tabular}{|c|c|c|}
\hline Sorted By & : & Signal \\
\hline Multiplier & : & 1.0000 \\
\hline Dilution & : & 1.0000 \\
\hline
\end{tabular}

Dilution

Use Multiplier \& Dilution Factor with ISTDs

Signal 1: VWD 1 A, Waveleng th=250 nm

\begin{tabular}{|c|c|c|c|c|c|c|}
\hline $\begin{array}{c}\text { Peak } \\
\#\end{array}$ & $\begin{array}{l}\text { RetTime } \\
\text { [min] }\end{array}$ & Type & $\begin{array}{l}\text { Width } \\
\text { [min] }\end{array}$ & $\begin{array}{c}\text { Area } \\
{\left[\mathrm{mhU}^{*} \mathrm{~s}\right]}\end{array}$ & $\begin{array}{l}\text { Height } \\
\text { [mÂU] }\end{array}$ & $\begin{array}{c}\text { Àrea } \\
\text { s. }\end{array}$ \\
\hline & & & & & & \\
\hline 1 & 22.615 & $\mathrm{BV}$ & 0.5689 & 1445.37256 & 39.04719 & 1.1154 \\
\hline 2 & 24.393 & VB & 0.7251 & $1.28137 \mathrm{e} 5$ & 2799.05127 & 98.8846 \\
\hline Tota & Ls : & & & $1.29582 \mathrm{e} 5$ & 2838.09846 & \\
\hline
\end{tabular}


Data File D: \DATA YANG JIAXIN YJX-2-170 YJX-2-170 2019-06-12 12-33-29\073-1601.D

Sample Name: zx-3-83-funan-RAC

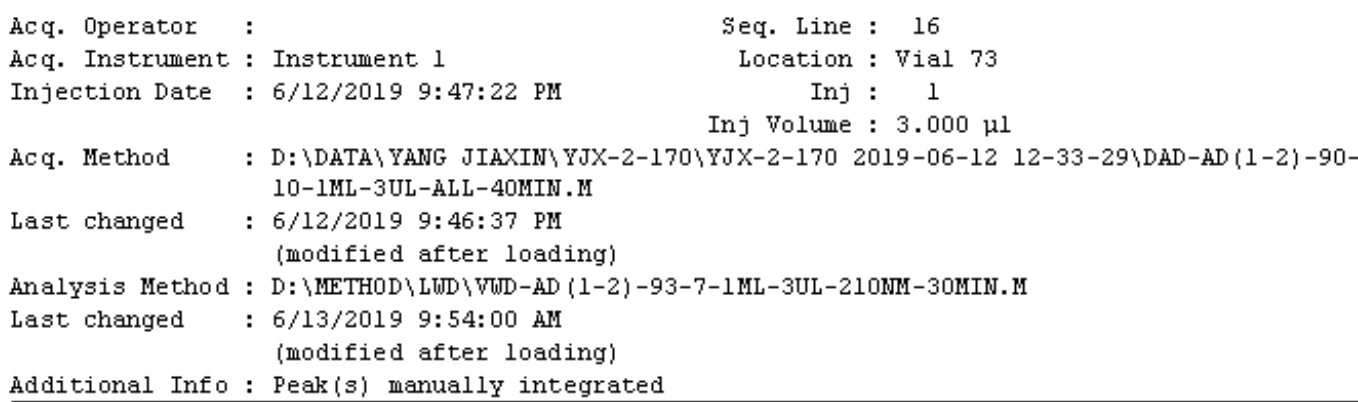

Acq. Method : D: \DATA YANG JIAXIN\YJX-2-170\YJX-2-170 2019-06-12 12-33-29\DAD-AD(1-2)-90$10-1 \mathrm{ML}-3 \mathrm{UL}-\mathrm{ALL}-40 \mathrm{MIN}$. M

Last changed : 6/12/2019 9:46:37 PM$$
\text { (modified after loading) }
$$

Analysis Method : D: \METHOD LLD \VWD-AD (1-2)-93-7-1ML-3UL-210MM-30MIN.M

Last changed : 6/13/2019 9:54:00 AM

(modified after loading)

Additional Info : Peak (s) manually integrated

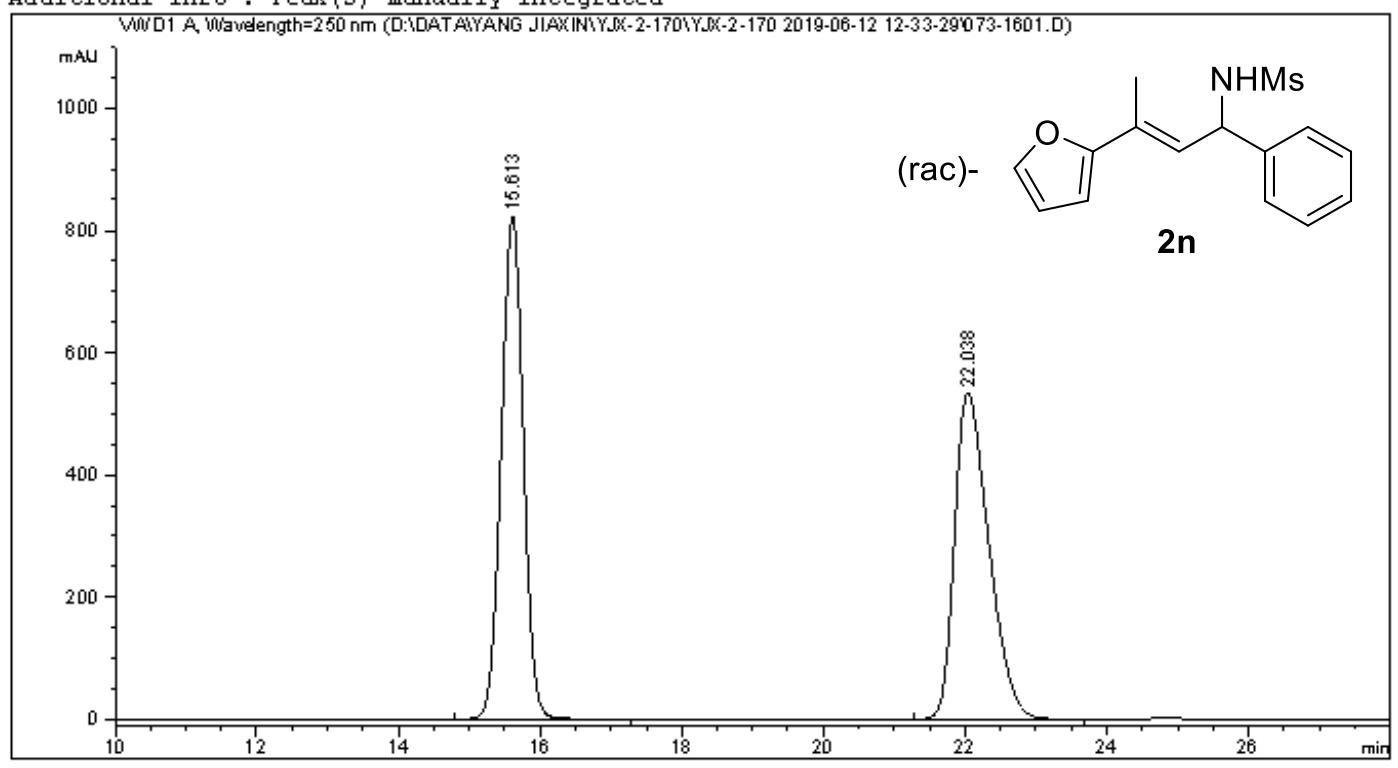

\section{Area Percent Report}

\begin{tabular}{|c|c|c|}
\hline Sorted By & : & Signal \\
\hline Multiplier & : & 1.0000 \\
\hline Dilution & : & 1.0000 \\
\hline
\end{tabular}

Use Multiplier \& Dilution Factor with IsTDs

Signal 1: VWDl A, Wavelength=250 nm

\begin{tabular}{|c|c|c|c|c|c|c|}
\hline $\begin{array}{c}\text { Peak } \\
\#\end{array}$ & $\begin{array}{c}\text { RetTime } \\
\text { [min] }\end{array}$ & Trpe & $\begin{array}{l}\text { Width } \\
\text { [min] }\end{array}$ & $\begin{array}{c}\text { Àrea } \\
{\left[\mathrm{m}^{2} \mathrm{U}^{*} \mathrm{~s}\right]}\end{array}$ & $\begin{array}{l}\text { Height } \\
\text { [mAU] }\end{array}$ & Àrea \\
\hline---1 & 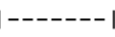 & & 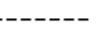 & ------- & ------ & -------- \\
\hline 1 & 15.613 & BB & 0.3378 & 1. $77972 \mathrm{e} 4$ & 821.41461 & 49.9084 \\
\hline 2 & 22.038 & $\mathrm{BB}$ & 0.5163 & 1. $78626 \mathrm{e} 4$ & 533.91852 & 50.0916 \\
\hline \multicolumn{4}{|c|}{ Totals : } & $3.56598 \mathrm{e} 4$ & 1355.33313 & \\
\hline
\end{tabular}


Data File D: \DATA YANG JIAXIN YJX-2-170 YJX-2-170 2019-06-12 12-33-29\074-1701.D

Sample Name: zx-3-83-funan

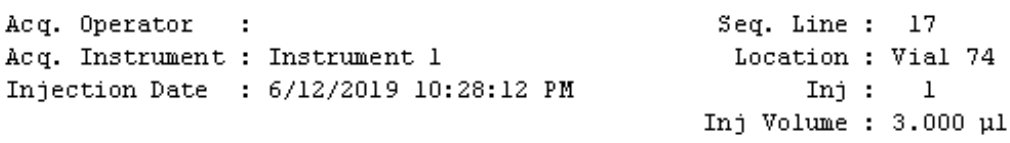

Acq. Method : D: \DATA YANG JIAXIN\YJX-2-170 YJX-2-170 2019-06-12 12-33-29\DAD-AD(1-2)-9010-1ML-3UL-ALL-40MIN. M

Last changed : 6/12/2019 9:46:37 PM

$$
\text { (modified after loading) }
$$

Analysis Method : D: \METHOD LLD \VWD-AD (1-2)-93-7-1ML-3UL-210MM-30MIN.M

Last changed : 6/13/2019 9:59:26 AM

(modified after loading)

Additional Info : Peak (s) manually integrated

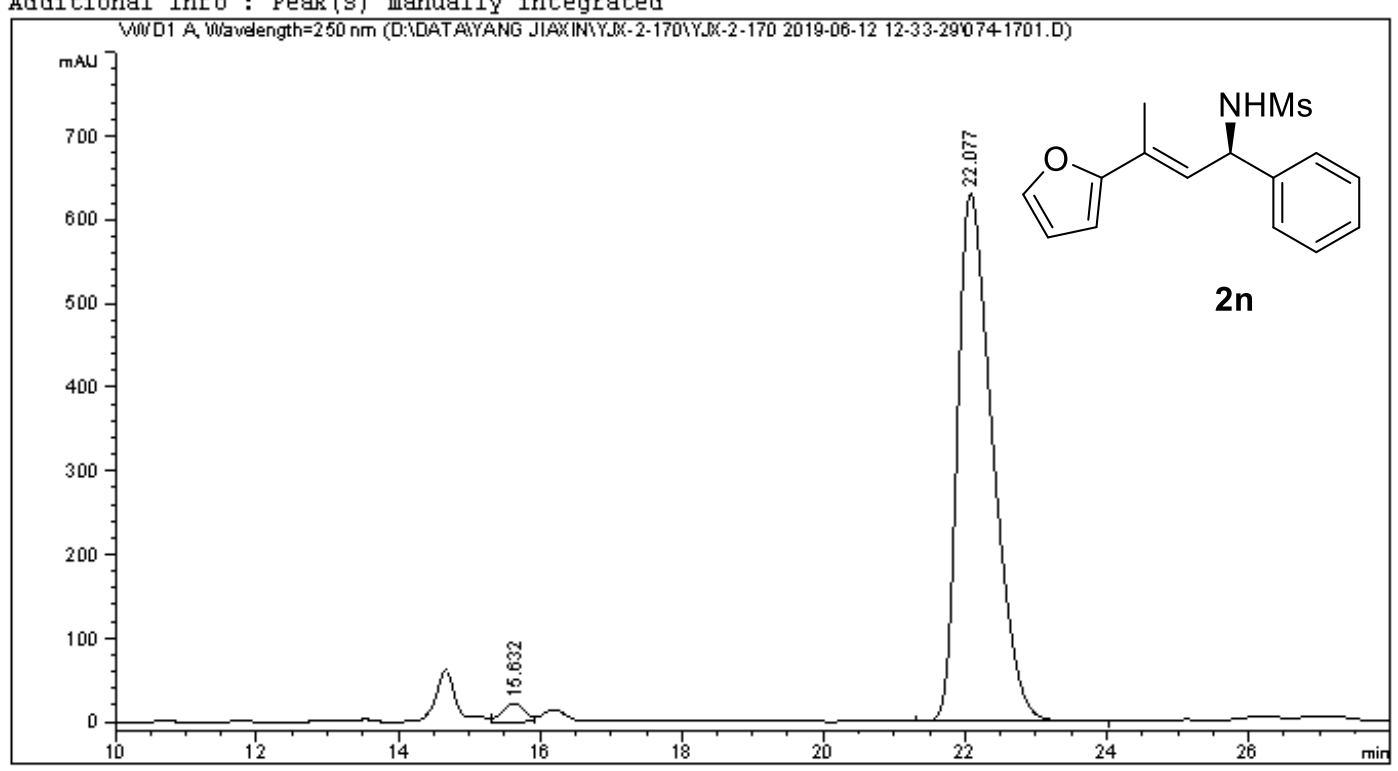

\begin{tabular}{|c|c|c|}
\hline Sorted BY & : & Signal \\
\hline Multiplier & : & 1.0000 \\
\hline Dilution & : & 1.000 \\
\hline
\end{tabular}

Use Multiplier \& Dilution Factor with ISTDs

Signal 1: VWD 1 A, Wavelength=250 nm

\begin{tabular}{|c|c|c|c|c|c|c|}
\hline $\begin{array}{c}\text { Peak } \\
\#\end{array}$ & $\begin{array}{c}\text { RetTime } \\
\text { [min] }\end{array}$ & Trpe & $\begin{array}{l}\text { Width } \\
\text { [min] }\end{array}$ & $\begin{array}{c}\text { Area } \\
\text { [mAUt*s] }\end{array}$ & $\begin{array}{l}\text { Height } \\
\text { [mAU] }\end{array}$ & Àrea \\
\hline-1 & 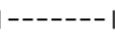 & & -- & ------- & --- & ------ \\
\hline 1 & 15.632 & VV & 0.3363 & 474.12067 & 21.58428 & 2.1534 \\
\hline 2 & 22.077 & $\mathrm{BB}$ & 0.5206 & $2.15437 \mathrm{e} 4$ & 630.58832 & 97.8466 \\
\hline \multicolumn{4}{|c|}{ Totals : } & $2.20178 \mathrm{e} 4$ & 652.17259 & \\
\hline
\end{tabular}


Data File D: \DATA \UAN YUQING $\backslash$ LK-A \LK-1906133 2019-06-13 18-32-32\071-1201.D Sample Name: ZX-3-83-WANJI-RAC
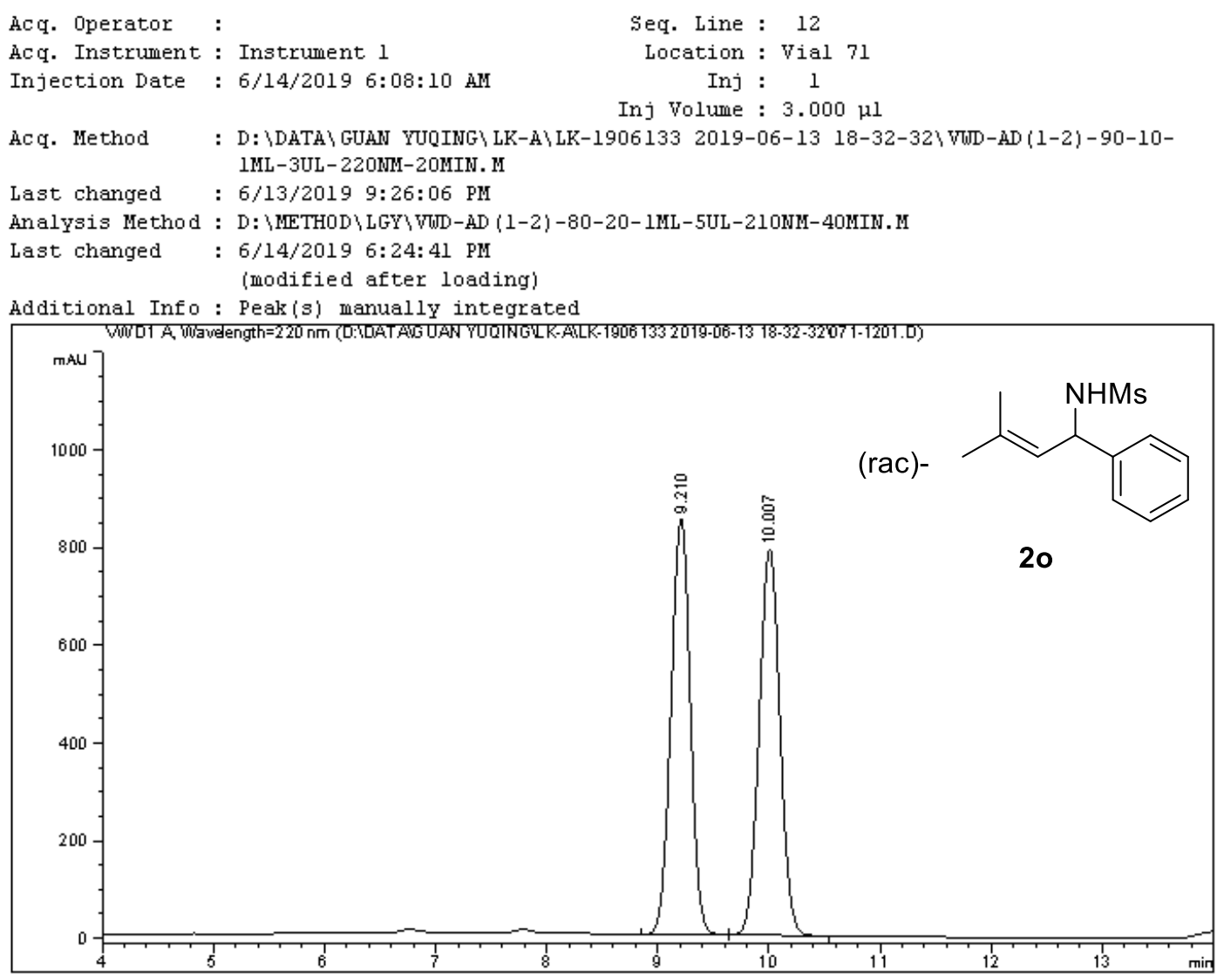

Area Percent Report

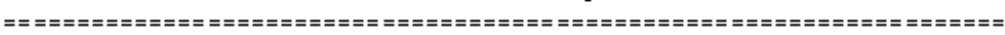

$\begin{array}{lll}\text { Sorted BY } & : & \text { Signal } \\ \text { Multiplier } & : & 1.0000 \\ \text { Dilution } & : & 1.0000\end{array}$

Use Multiplier \& Dilution Factor with ISTDs

Signal 1: VWDl A, Wavelength=220 nm

\begin{tabular}{|c|c|c|c|c|c|c|}
\hline $\begin{array}{c}\mathrm{Peak} \\
\#\end{array}$ & $\begin{array}{c}\text { RetTime } \\
\text { [min] }\end{array}$ & Type & $\begin{array}{l}\text { Width } \\
\text { [min] }\end{array}$ & $\begin{array}{c}\text { Area } \\
{\left[\text { [mAU } U^{*} \Omega\right]}\end{array}$ & $\begin{array}{l}\text { Height } \\
\text { [mAU] }\end{array}$ & $\begin{array}{c}\text { Area } \\
\stackrel{8}{*}\end{array}$ \\
\hline & & & & ------ & $-0-0-1$ & $=-$ \\
\hline 1 & 9.210 & $\mathrm{BB}$ & 0.1880 & $1.02154 \mathrm{e} 4$ & 851.11835 & 49.9060 \\
\hline 2 & 10.007 & $\mathrm{BB}$ & 0.2035 & $1.02539 \mathrm{e} 4$ & 789.50665 & 50.0940 \\
\hline Tot & : & & & $2.04693 \mathrm{e} 4$ & 1640.62500 & \\
\hline
\end{tabular}


Data File D: \DATA \GUAN YUQING $\backslash$ LK-A \LK-1906133 2019-06-13 18-32-32\072-1301.D Sample Name: $2 X-3-83-$ WhNJI
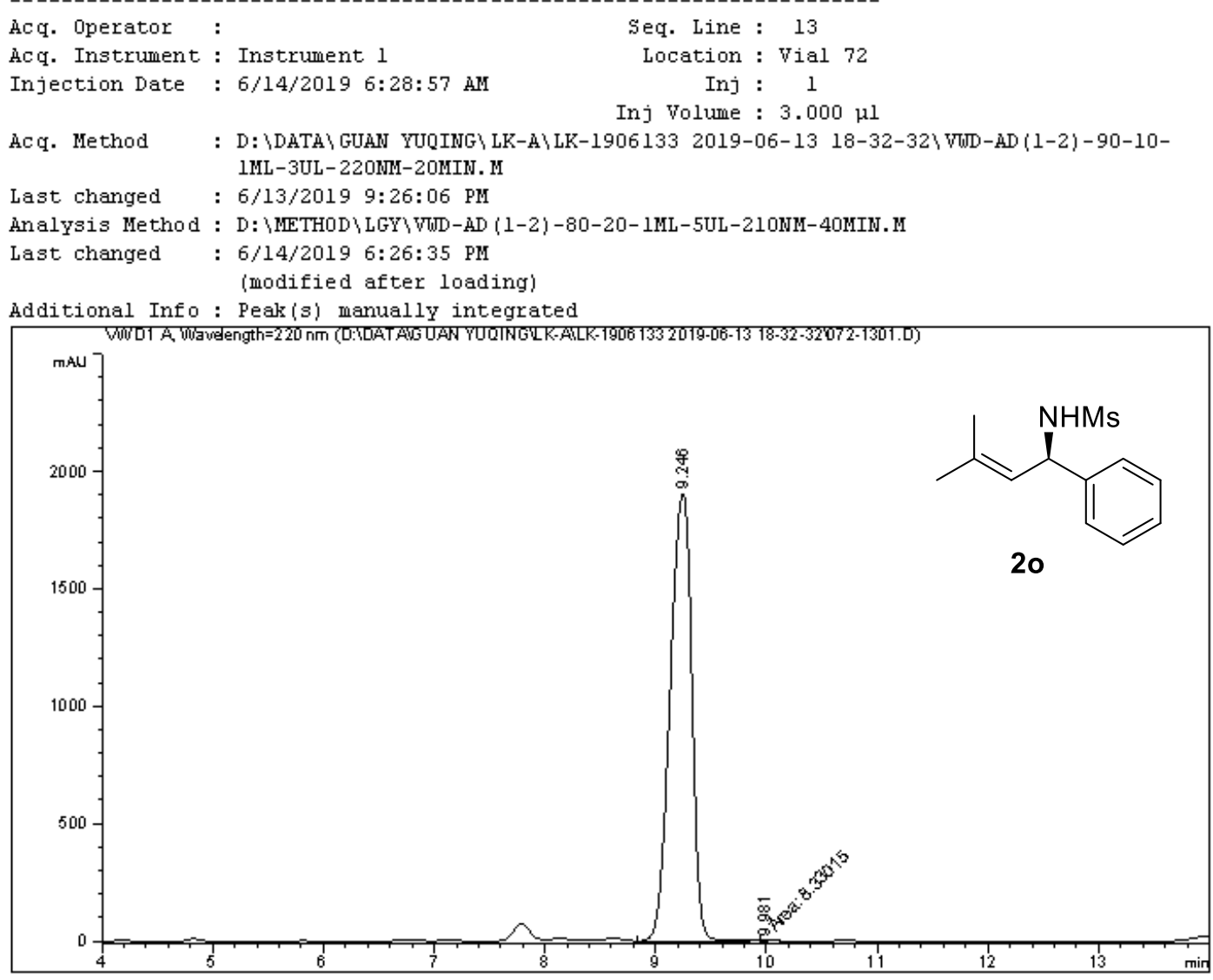

Area Percent Report

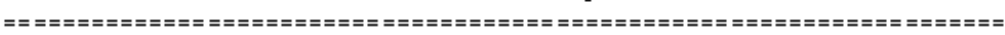

$\begin{array}{lcc}\text { Sorted By } & : & \text { Signal } \\ \text { Multiplier } & : & 1.0000 \\ \text { Dilution } & : & 1.0000 \\ \text { Use Multiplier } & \text { \& Dilution } & \text { Factor with }\end{array}$

Signal 1: VWDl A, Wavelength=220 nm

\begin{tabular}{|c|c|c|c|c|c|}
\hline $\begin{array}{c}\text { Peak } \\
\#\end{array}$ & $\begin{array}{l}\text { RetTime Type } \\
\text { [min] }\end{array}$ & $\begin{array}{l}\text { Width } \\
\text { [min] }\end{array}$ & 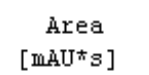 & $\begin{array}{l}\text { Height } \\
\text { [mAU] }\end{array}$ & $\begin{array}{c}\text { Area } \\
\stackrel{s}{*}\end{array}$ \\
\hline- & & & --- & - & \\
\hline 1 & & & $2.57243 \mathrm{e} 4$ & 1903 & 9676 \\
\hline 2 & .981 & 0.1085 & 8.33015 & 1.38898 & 0.0324 \\
\hline
\end{tabular}

Totals : $\quad 2.57326 \mathrm{e} 4 \quad 1904.80365$ 
Data File D: \DATA $2 X \backslash Z X-3-83 \backslash 2 X-3-81$ 2019-06-15 14-39-38\071-0401.D

Sample Name: $2 X-3-81-N o M e-R A C$

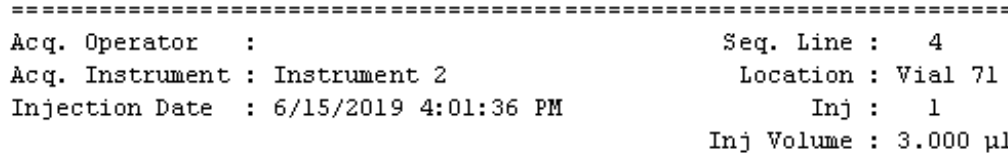

Acq. Method : D: \DATA $\backslash Z X \backslash Z X-3-83 \backslash Z X-3-81$ 2019-06-15 14-39-38\DAD-0D (1-2)-90-10-1ML-3ULALL-60MIN-0615.M

Last changed : 6/15/2019 3:59:12 PM

Analysis Method : D: \METHOD $\backslash X \backslash D A D-0 D(1-2)-90-10-1 M L-3 U L-A L L-60 M I N-0615 . M$

Last changed : 6/15/2019 8:44:37 PM

(modified after loading)

Additional Info : Peak (s) manually integrated

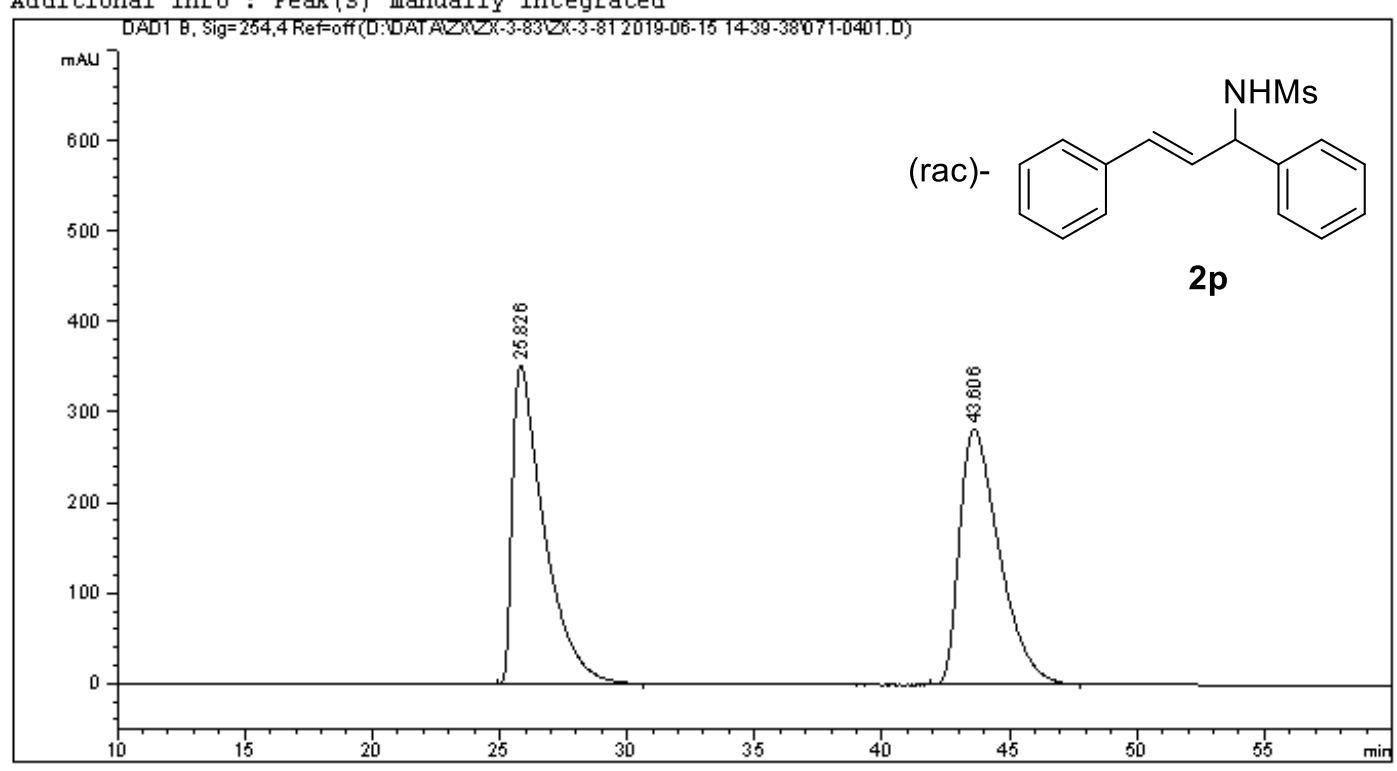

Area Percent Report

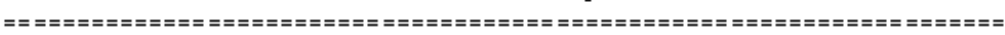

$\begin{array}{lll}\text { Sorted BY } & : & \text { Signal } \\ \text { Multiplier } & : & 1.0000 \\ \text { Dilution } & : & 1.0000\end{array}$

Use Multiplier \& Dilution Factor with IsTDs

Signal 1: DADl B, Sig=254, 4 Ref=off

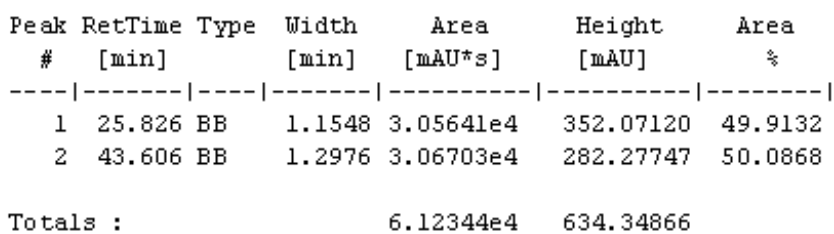


Data File D: \DATA $\backslash X \backslash Z X-3-83 \backslash Z X-3-81$ 2019-06-15 14-39-38\072-0501.D

Sample Name: $2 X-3-83-$ NoMe
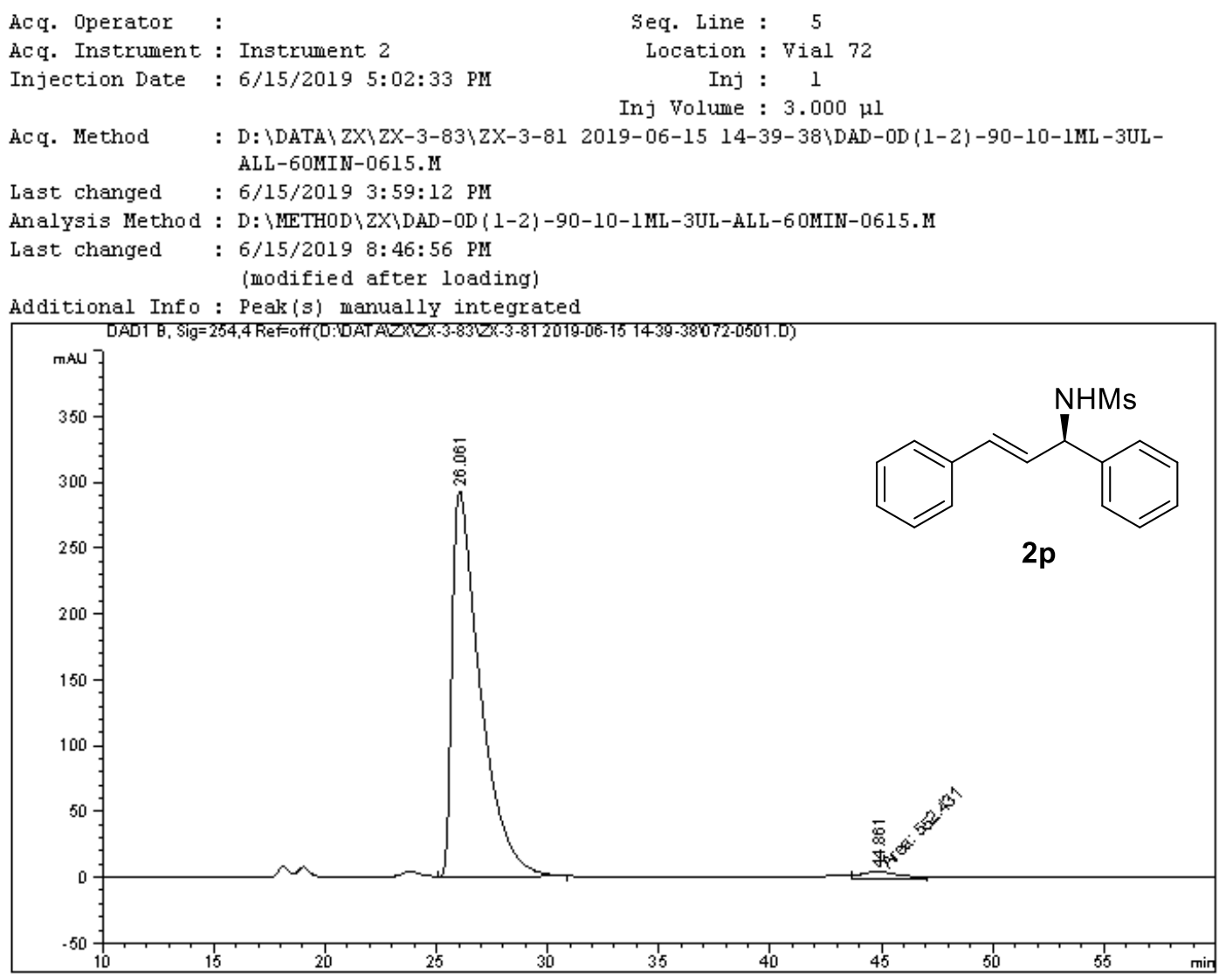

Area Percent Report

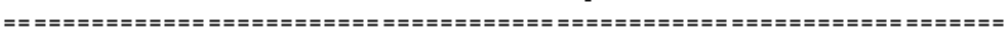

$\begin{array}{lll}\text { Sorted By } & : & \text { signal } \\ \text { Multiplier } & : & 1.0000 \\ \text { Dilution } & : & 1.0000\end{array}$

Use Multiplier \& Dilution Factor with ISTDs

Signal 1: DADl B, Sig=254, 4 Ref=off

\begin{tabular}{|c|c|c|c|c|c|}
\hline $\begin{array}{c}\text { eak } \\
\#\end{array}$ & $\begin{array}{l}\text { RetTime Type } \\
\text { [min] }\end{array}$ & $\begin{array}{l}\text { Width } \\
\text { [min] }\end{array}$ & $\begin{array}{c}\text { Area } \\
\text { [mAUt* } \mathrm{m}]\end{array}$ & $\begin{array}{l}\text { Height } \\
\text { [m̉U] }\end{array}$ & $\begin{array}{c}\text { Àrea } \\
\stackrel{8}{*}\end{array}$ \\
\hline 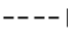 & & & 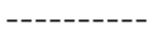 & & -- \\
\hline 1 & & 0 & $2.57438 \mathrm{e} 4$ & 293.00894 & 3992 \\
\hline 2 & 44 & 659 & 552.43103 & 4.68338 & 2.1008 \\
\hline
\end{tabular}

Totals : $\quad 2.62962 \mathrm{e} 4 \quad 297.69232$ 
Data File D: \DATA $\backslash$ LTD $\backslash L W D-5-160-13 \backslash L$ LD-5-160-13 2019-07-22 08-05-06\031-0401.D Sample Name: LK-190722-ZX-RAC

\begin{tabular}{|c|c|c|}
\hline Acq. Operator & : & seq. Line : \\
\hline Ac q. Instrument & : Instrument 1 & Location : Vial 31 \\
\hline Injection Date & : 7/22/2019 9:12:03 AM & $\begin{array}{rlc}\text { Inj : } & 1 \\
\text { Inj Volume } & 5 & 000\end{array}$ \\
\hline
\end{tabular}

Acq. Method : D: \DATA \LTD $\backslash L W D-5-160-13 \backslash L W D-5-160-132019-07-22$ 08-05-06\VID-AD (1-2)-95-51 ML-5UL-210MM-80MIN. M

Last changed : 12/25/2018 3:16:16 PM

Analysis Method : D: \METHOD GUAN YUQING VUD-AD (1-2)-95-5-1ML-5UL-210MM-15MIN.M

Last changed : 7/22/2019 2:43:02 PM

(modified after loading)

Additional Info: Peak (s) manually integrated

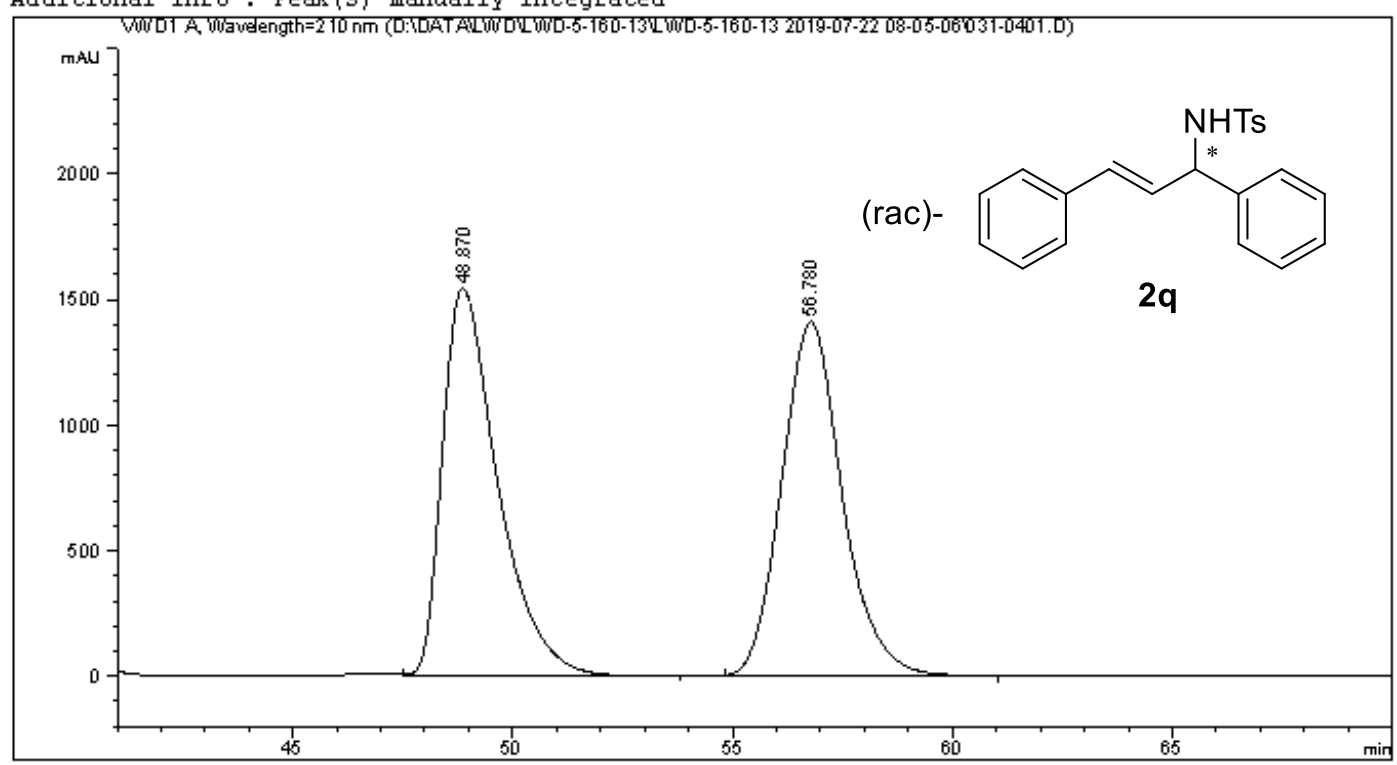

Area Percent Report

\begin{tabular}{|c|c|c|}
\hline Sorted By & : & Signal \\
\hline Multiplier & : & 1.0000 \\
\hline Dilution & : & 1.0000 \\
\hline
\end{tabular}

Use Multiplier \& Dilution Factor with ISTDs

Signal 1: VWDl A, Waveleng th=210 nm

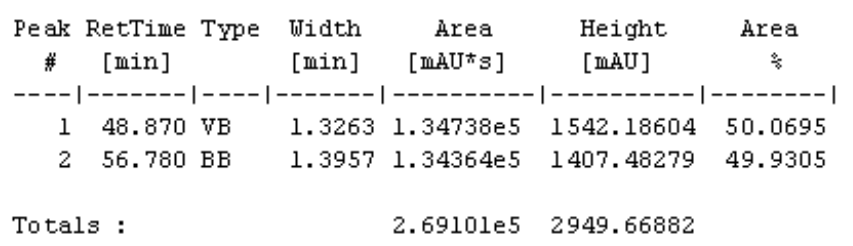


Data File D: \DATA $\backslash$ LD $\backslash L W D-5-160-13 \backslash L W D-5-160-13$ 2019-07-22 08-05-06 032-0501.D Sample Name: LK-190722-ZX-EE

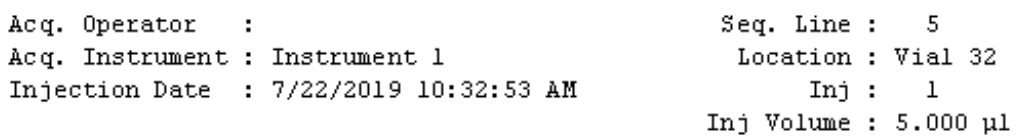

Ac q. Method : D: \DATA $\backslash$ LWD $\backslash L W D-5-160-13 \backslash$ LWD-5-160-13 2019-07-22 08-05-06 VVDD-AD (1-2) -95-5$1 \mathrm{ML}-5 \mathrm{UL}-210 \mathrm{MM}-80 \mathrm{MIN}$. M

Last changed : 12/25/2018 3:16:16 PM

Analysis Method : D: \METHOD \GUAN YUQING VUDD-AD (1-2)-95-5-1ML-5UL-210MM-15M IN. M

Last changed : 7/22/2019 2:41:05 PM

(modified after loading)

Additional Info : Peak (s) manually integrated

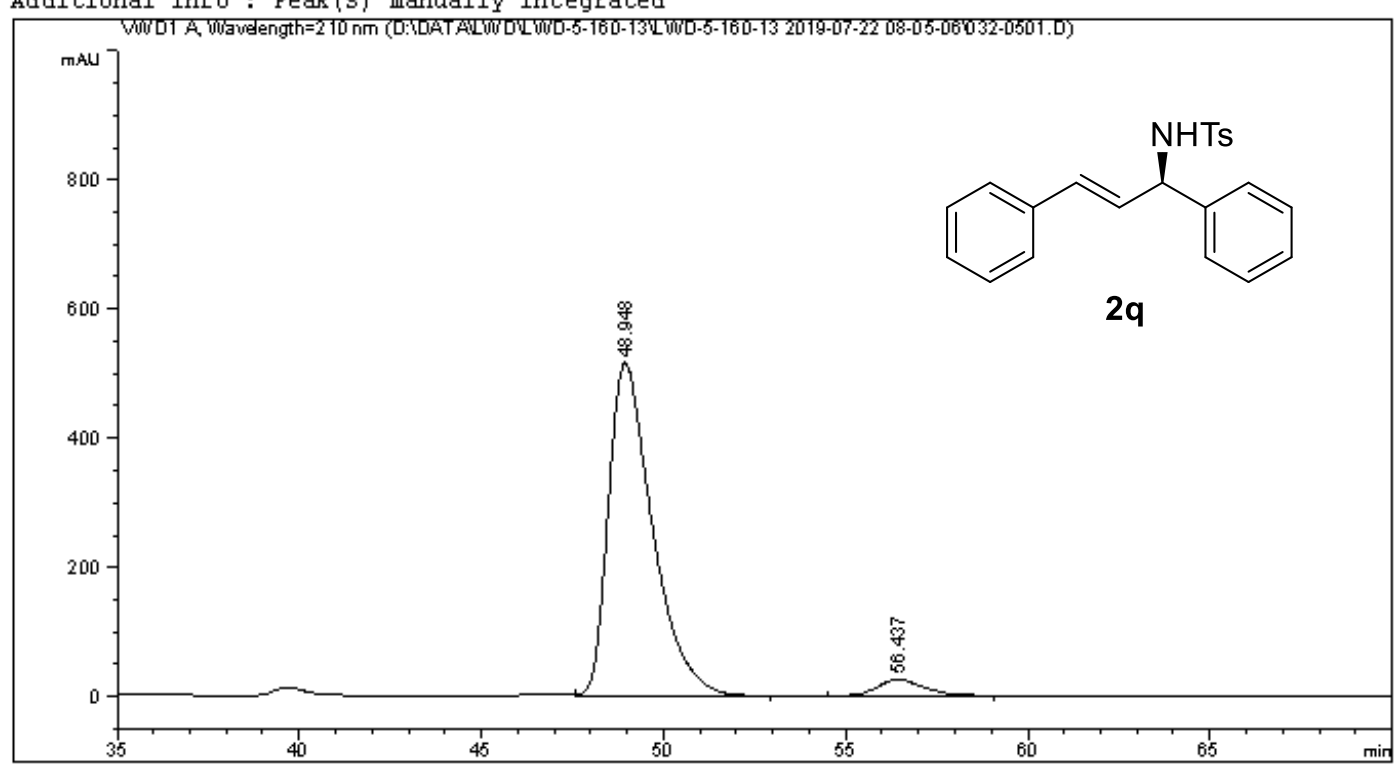

Area Percent Report

\begin{tabular}{|c|c|c|}
\hline Sorted By & : & Signal \\
\hline Multiplier & : & 1.0000 \\
\hline Dilution & : & 1.0000 \\
\hline
\end{tabular}

Use Multiplier \& Dilution Factor with IsTDs

Signal 1: VWD 1 , Waveleng th=210 nm

\begin{tabular}{|c|c|c|c|c|c|c|}
\hline $\begin{array}{c}\mathrm{Peak} \\
\#\end{array}$ & $\begin{array}{c}\text { RetTime } \\
\text { [min] }\end{array}$ & Type & $\begin{array}{l}\text { Width } \\
\text { [min] }\end{array}$ & $\begin{array}{c}\text { Area } \\
\text { [mAUt's] }\end{array}$ & $\begin{array}{l}\text { Height } \\
\text { [mAU] }\end{array}$ & $\begin{array}{c}\text { Area } \\
\stackrel{4}{*}\end{array}$ \\
\hline-- & & & & ---- & -- & -------1 \\
\hline 1 & 48.948 & $v B$ & 1.2704 & $4.34766 \mathrm{e} 4$ & 516.34009 & 94.8651 \\
\hline 2 & 56.437 & $\mathrm{BB}$ & 1.2956 & 2353.31494 & 26.13412 & 5.1349 \\
\hline
\end{tabular}

Totals : $\quad 4.58299 \mathrm{e} 4 \quad 542.47420$ 